Supporting Information for

\title{
Streamlined Preparation and Coordination Chemistry of Hybrid Phosphine-Phosphaalkene Ligands
}

\author{
Kevin W. Magnuson, ${ }^{1}$ Shelly M. Oshiro, ${ }^{1}$ Joshua R. Gurr, ${ }^{1}$ Wesley Y. Yoshida, ${ }^{1}$ Milan \\ Gembicky, ${ }^{3}$ Arnold L. Rheingold, ${ }^{3}$ Russell P. Hughes, ${ }^{2}$ and Matthew F. Cain ${ }^{*}, 1$ \\ ${ }^{1}$ Department of Chemistry, University of Hawai'i at Mānoa, 2545 McCarthy Mall, Honolulu, \\ Hawaii 96822 \\ ${ }^{2} 6128$ Burke Laboratory, Department of Chemistry, Dartmouth College, Hanover, New \\ Hampshire 03755 \\ ${ }^{3}$ Department of Chemistry, University of California, San Diego, 9500 Gilman Drive, La Jolla, \\ California 92093
}

Contact: mfcain@hawaii.edu

1. General Experimental Details

2. Experimental Details for the Synthesis of New Compounds (including NMR spectra and references)

3. DFT Computational Details

4. Experimental Details for Crystal Structure Refinement and Acquisition (including references) 
General Experimental Details All manipulations were conducted under a nitrogen or argon atmosphere in a MBRAUN drybox or using Schlenk techniques unless otherwise specified. All glassware was oven-dried prior to use. Anhydrous grade acetonitrile, pentane, toluene, benzene, ether, dichloromethane, and THF were purchased from commercial suppliers (Aldrich or Acros) and pumped directly into the glove box. All solvents were stored over ovenactivated $4 \AA$ molecular sieves (Aldrich). The following compounds were purchased from commercial suppliers and used as is: $\mathrm{PHCy}_{2}, \mathrm{PHPh}_{2}, \mathrm{Zn}$ dust, $\mathrm{Pt}_{2}\left(\mathrm{Et}_{2} \mathrm{~S}\right)_{2} \mathrm{Cl}_{2}, \mathrm{Pd}(\mathrm{COD}) \mathrm{Cl}_{2}$, and bromoacetaldehyde diethyl acetal. $\mathrm{PMe}_{3}$ was purchased from Strem and dried over molecular sieves prior to use. $\mathrm{Mes}^{*} \mathrm{PCl}_{2}$ was prepared using the published literature protocol. ${ }^{1}$ NMR spectra were obtained on Varian spectrometers operating at either $300 \mathrm{MHz}$ or $500 \mathrm{MHz}$; all spectra below were recorded on the $500 \mathrm{MHz}$ NMR spectrometer. NMR chemical shifts are reported as ppm relative to tetramethylsilane, and are referenced to the residual proton or ${ }^{13} \mathrm{C}$ signal of the solvent $\left({ }^{1} \mathrm{H} \mathrm{CDCl}_{3}: 7.27 \mathrm{ppm},{ }^{1} \mathrm{H} \mathrm{C}_{6} \mathrm{D}_{6}: 7.16 \mathrm{ppm} ;{ }^{13} \mathrm{C} \mathrm{CDCl}_{3}: 77.16 \mathrm{ppm},{ }^{13} \mathrm{C}\right.$ $\left.\mathrm{C}_{6} \mathrm{D}_{6}, 128.06 \mathrm{ppm}\right)$. Mass spectrometry was conducted at the University of California at Irvine (Dr. J Greaves) on compounds $\boldsymbol{E}-\mathbf{1 a}, \boldsymbol{E}-\mathbf{1 b}, \mathbf{2 a}$, and 3a. Mass spectrometry on $\mathbf{2 b}$ and $\mathbf{3 b}$ was conducted at the University of Hawai'i at Mānoa using a new Agilent 6545 Accurate-Mass QTOF LC/MS (NSF CHE-1532310). Analytical data were obtained from the CENTC Elemental Analysis Facility at the University of Rochester, funded by NSF CHE-0650456. All X-ray quality crystals were analyzed at the Small Molecule X-ray Crystallography Facility located at the University of California at San Diego.

1 Cowley, A.H.; Norman, N.C.; Pakulski, M. Inorg. Synth. 1990, 27, 235-240. 


\section{Experimental Details for the Synthesis of New Compounds Synthesis of $\mathrm{Cy}_{2} \mathrm{P}-\mathrm{CH}_{2}-\mathrm{C}(\mathrm{H})=\mathrm{PMes}^{*}(\mathrm{E}-1 \mathrm{a})$}

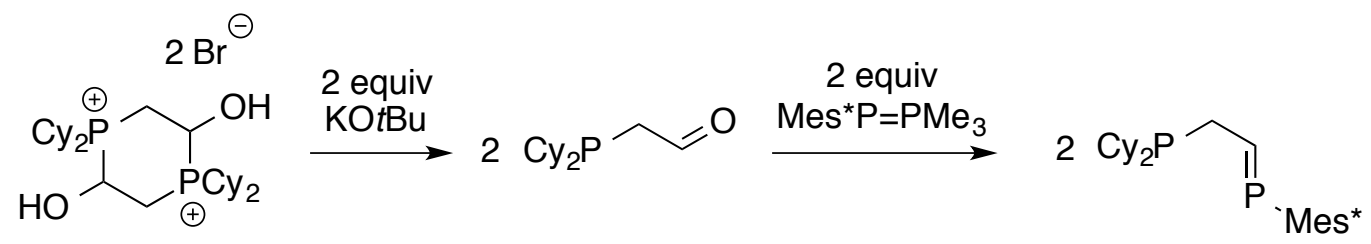

Mes* $\mathrm{PCl}_{2}$ (500 mg, $1.44 \mathrm{mmol}$ ) and $\mathrm{Zn}$ dust ( $470 \mathrm{mg}, 7.19 \mathrm{~mol}, 5$ equiv relative to $\mathrm{Mes}^{*} \mathrm{PCl}_{2}$ ) were loaded into a vial and $6 \mathrm{~mL}$ of benzene was added. The heterogeneous mixture was cooled to $-35{ }^{\circ} \mathrm{C}$. A solution of $\mathrm{PMe}_{3}\left(285 \mathrm{mg}, 3.75 \mathrm{mmol}, 2.6\right.$ equiv relative to $\mathrm{Mes}^{*} \mathrm{PCl}_{2}$ ) in $4 \mathrm{~mL}$ of benzene was then added to the frozen mixture. The reaction mixture was warmed to RT and stirred for $2 \mathrm{~h}$. After $2 \mathrm{~h}$, the heterogeneous reaction mixture was filtered through a Celite plug affording a bright yellow filtrate. Concurrently, the phosphonium dimer (460 mg, $0.716 \mathrm{mmol}$ ) and $\mathrm{KOtBu}(162 \mathrm{mg}, 1.44 \mathrm{mmol}$ ) were combined into a separate vial, followed by the addition of $5 \mathrm{~mL}$ of toluene. Manual stirring of the reaction mixture resulted in increased clarity/transparency of the heterogeneous solution. After 15 min of stirring, the reaction mixture was filtered into the same vessel as the yellow filtrate. The mixed toluene/benzene reaction mixture was then transferred to a Schlenk bomb, and the screw-top Teflon cap was sealed. The bomb was brought outside of the glove box and heated to $40{ }^{\circ} \mathrm{C}$ overnight $(12 \mathrm{~h})$. The homogeneous yellow reaction mixture was then cooled to RT, brought back inside the glove box, and concentrated under vacuum. The crude product mixture was dissolved in $20 \mathrm{~mL}$ of pentane and filtered through a Celite plug to remove the $\mathrm{PMe}_{3}(\mathrm{O})$ (Note: It is critically important to remove all of the benzene. If it is not completely removed some of the $\mathrm{PMe}_{3}(0)$ is solubilized and NOT removed by filtration). The pale yellow filtrate was then concentrated under vacuum affording a sticky solid. Recrystallization of the crude product from a $5 \mathrm{~mL}$ of 25:75 solution of THF and acetonitrile resulted in the precipitation of the desired product as a pale yellow crystalline solid (531 mg, $1.06 \mathrm{mmol}, 74 \%$ ). X-ray quality crystals could also be grown in this fashion.

Anal. Calcd. for $\mathrm{C}_{32} \mathrm{H}_{54} \mathrm{P}_{2}$ : C, 76.76; $\mathrm{H}, 10.87$. Found: $\mathrm{C}, 76.93 ; \mathrm{H}, 11.12$. HRMS $\mathrm{m} / z$ calcd for $\mathrm{C}_{32} \mathrm{H}_{55} \mathrm{P}_{2}\left(\mathrm{MH}^{+}\right):$501.3779. Found: 501.3775. ${ }^{31} \mathrm{P}\left\{{ }^{1} \mathrm{H}\right\}$ NMR $\left(\mathrm{C}_{6} \mathrm{D}_{6}\right): \delta 250.6(\mathrm{~d}, J=37$, $\mathrm{P}=\mathrm{C}),-2.4\left(\mathrm{~d}, J=37, \mathrm{PCy}_{2}\right) .{ }^{1} \mathrm{H}$ NMR $\left(\mathrm{C}_{6} \mathrm{D}_{6}\right): \delta$ 7.68-7.58 (overlapping dtd with Mes*, $3 \mathrm{H}$, $\mathrm{P}=\mathrm{CH}$ and $\mathrm{Mes}^{*}$ ), 2.74 (ddd, $J=2.5,9.5,22,2 \mathrm{H}, \mathrm{CH}_{2}$ backbone), 1.80-1.54 (overlapping $\mathrm{Cy}$ region, $12 \mathrm{H}, \mathrm{Cy}), 1.68(18 \mathrm{H}, t-\mathrm{Bu}), 1.31(9 \mathrm{H}, t-\mathrm{Bu}), 1.26-1.11$ (overlapping multiplet, $10 \mathrm{H}$, Cy). ${ }^{13} \mathrm{C}\left\{{ }^{1} \mathrm{H}\right\}$ NMR $\left(\mathrm{C}_{6} \mathrm{D}_{6}\right): \delta 178.0(\mathrm{dd}, J=37,10, \mathrm{P}=\mathrm{C}), 154.3(\mathrm{Ar}), 149.7(\mathrm{Ar}), 140.0(\mathrm{~d}, J=$ 57, $\mathrm{Ar}$ ), 122.0 ( $\mathrm{Ar}$ ), $38.6\left(\mathrm{CMe}_{3}\right), 35.0\left(\mathrm{CMe}_{3}\right), 34.1(\mathrm{~d}, J=7, t-\mathrm{Bu}), 33.8$ (d, $J=18, \mathrm{P}-\mathrm{CH}(\mathrm{Cy})$ ), $31.5(t-\mathrm{Bu}), 30.1(\mathrm{~d}, J=12, \mathrm{Cy}), 29.4\left(\mathrm{dd}, J=22,27, \mathrm{CH}_{2}\right.$ backbone), $27.6(\mathrm{t}, J=10, \mathrm{Cy}), 26.8$ (Cy). ${ }^{13} \mathrm{C}$ NMR assignments were aided by DEPT experiments. 
NMR Spectroscopy of $\boldsymbol{E}$-1a

${ }^{31} \mathrm{P}\left\{{ }^{1} \mathrm{H}\right\}$ NMR $\left(\mathrm{C}_{6} \mathrm{D}_{6}\right)$

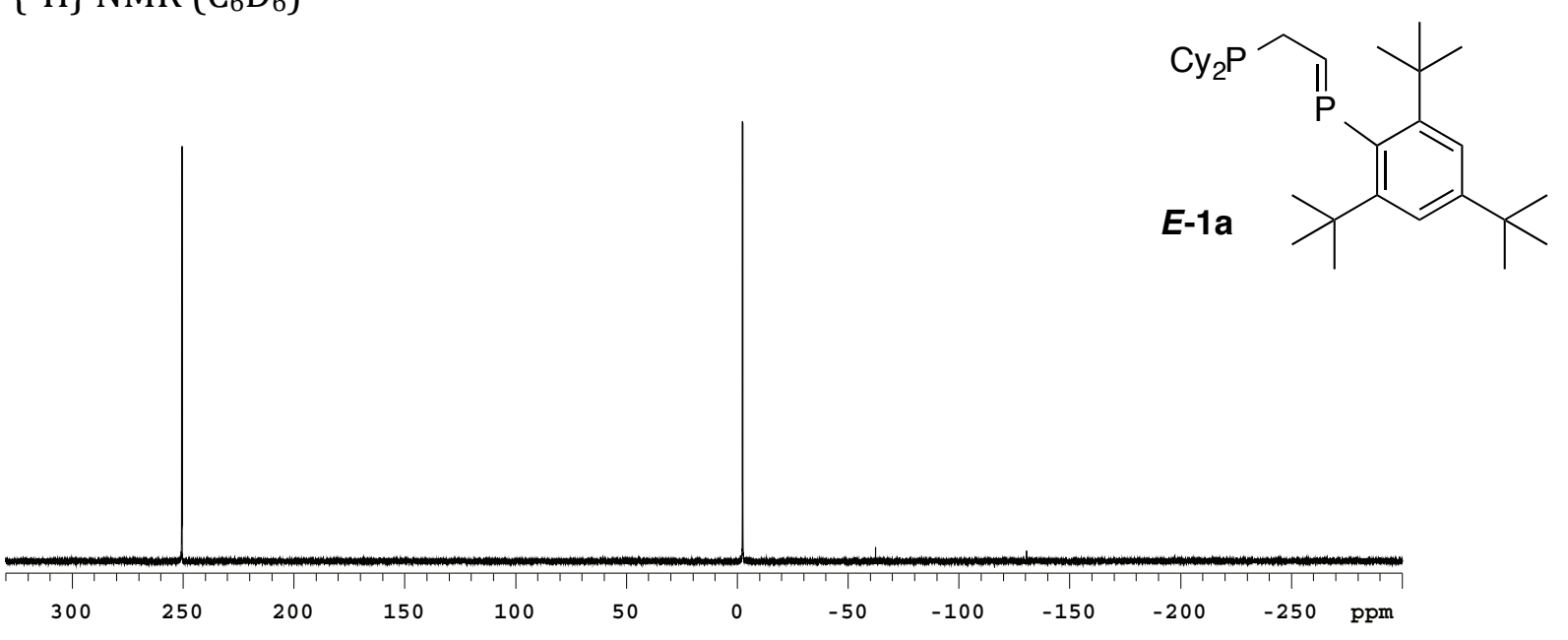

${ }^{31} \mathrm{P}\left\{{ }^{1} \mathrm{H}\right\}$ NMR $\left(\mathrm{C}_{6} \mathrm{D}_{6}\right)$ : Selected Zoom

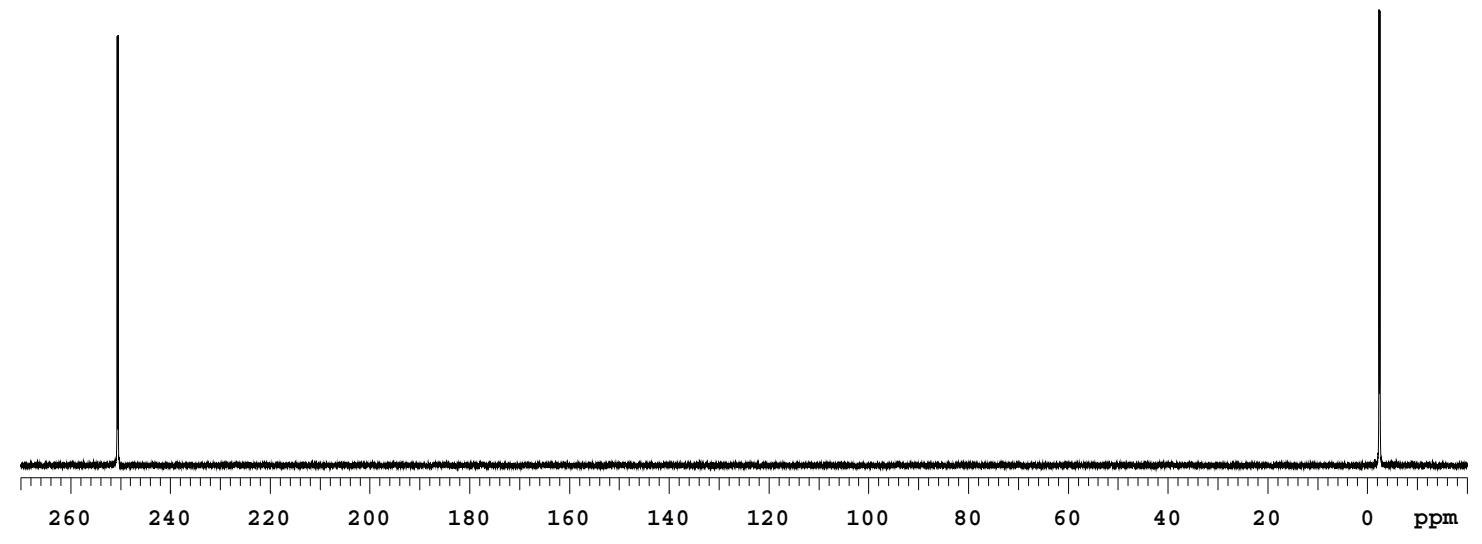


${ }^{1} \mathrm{H}$ NMR $\left(\mathrm{C}_{6} \mathrm{D}_{6}\right)$

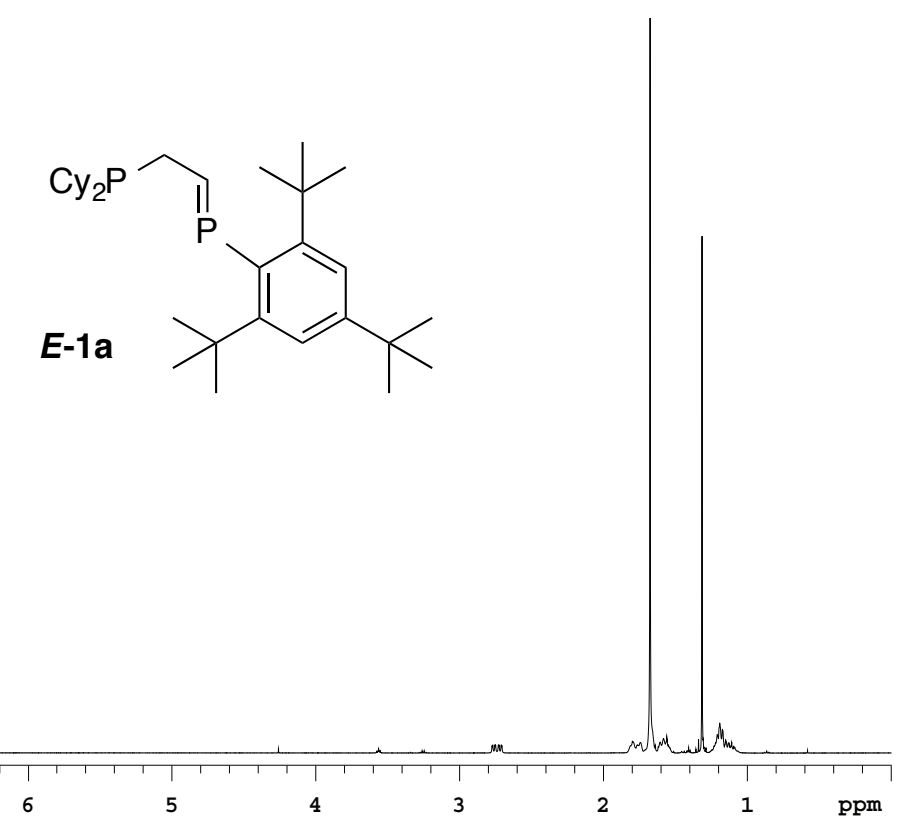

${ }^{1} \mathrm{H}$ NMR $\left(\mathrm{C}_{6} \mathrm{D}_{6}\right)$ : Selected Zoom

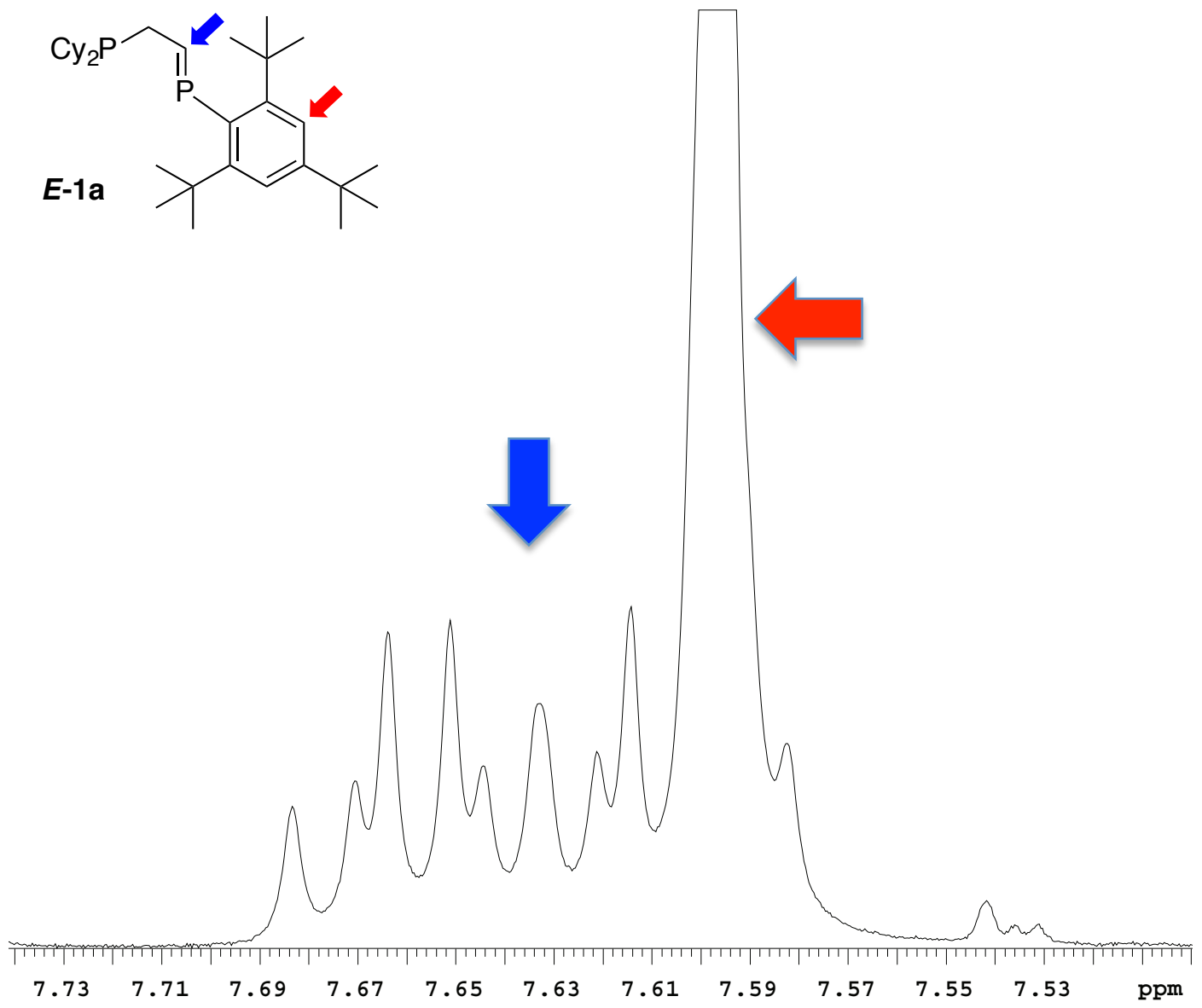


${ }^{1} \mathrm{H}$ NMR $\left(\mathrm{C}_{6} \mathrm{D}_{6}\right)$ : Selected Zoom

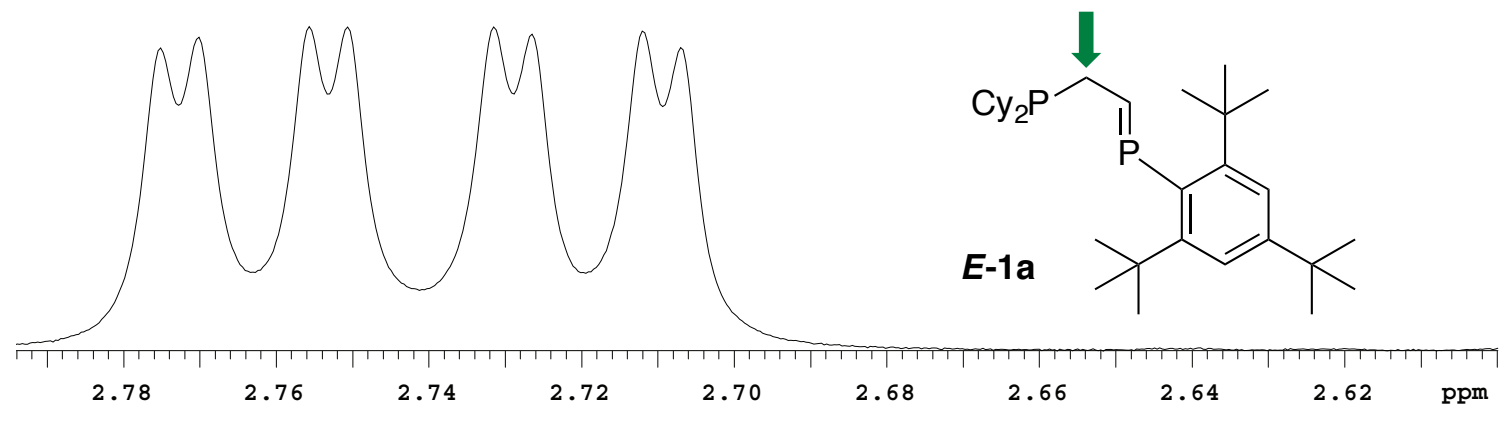

${ }^{13} \mathrm{C}\left\{{ }^{1} \mathrm{H}\right\}$ NMR $\left(\mathrm{C}_{6} \mathrm{D}_{6}\right)$

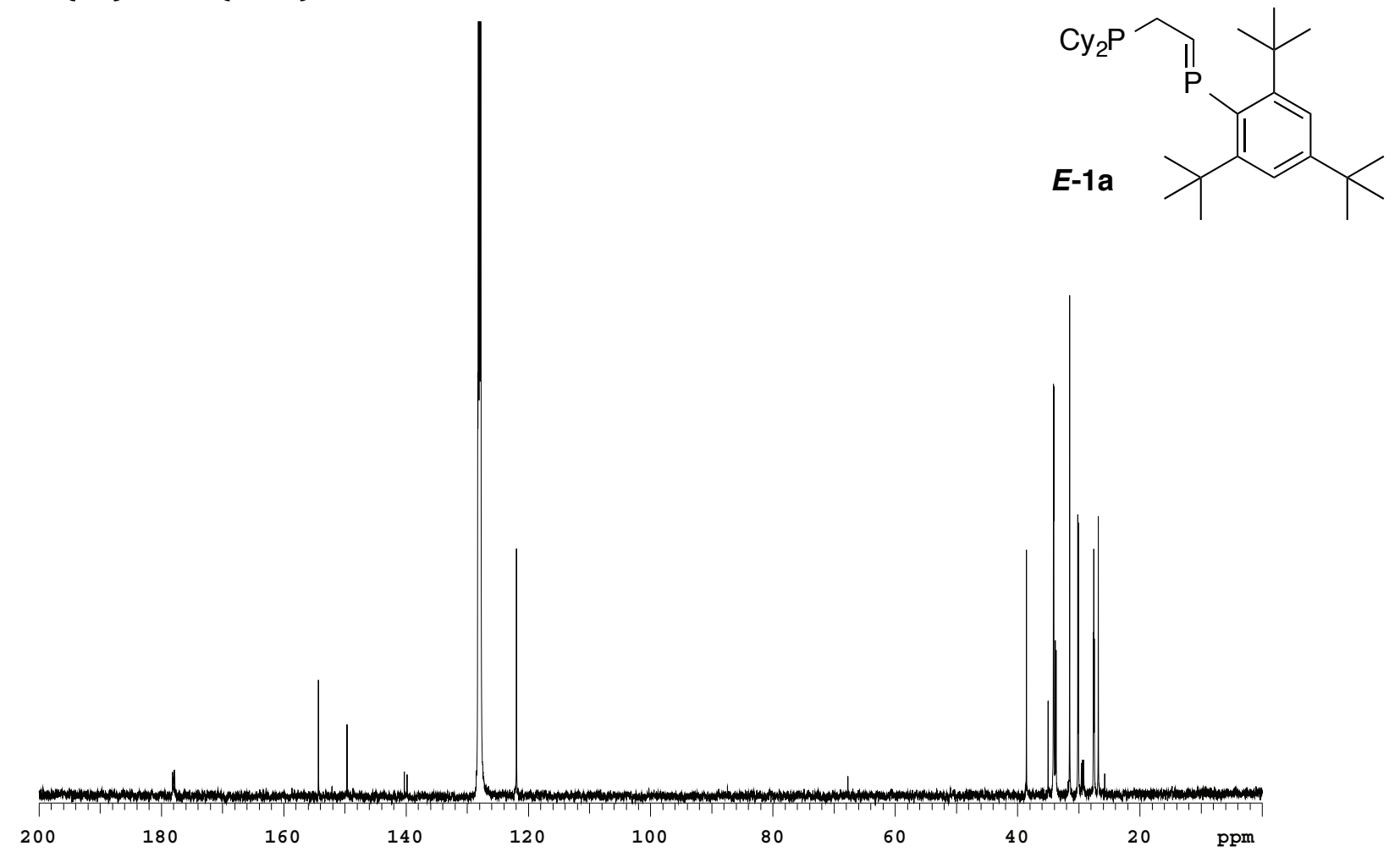


${ }^{13} \mathrm{C}\left\{{ }^{1} \mathrm{H}\right\}$ NMR $\left(\mathrm{C}_{6} \mathrm{D}_{6}\right)$ : Selected Zoom

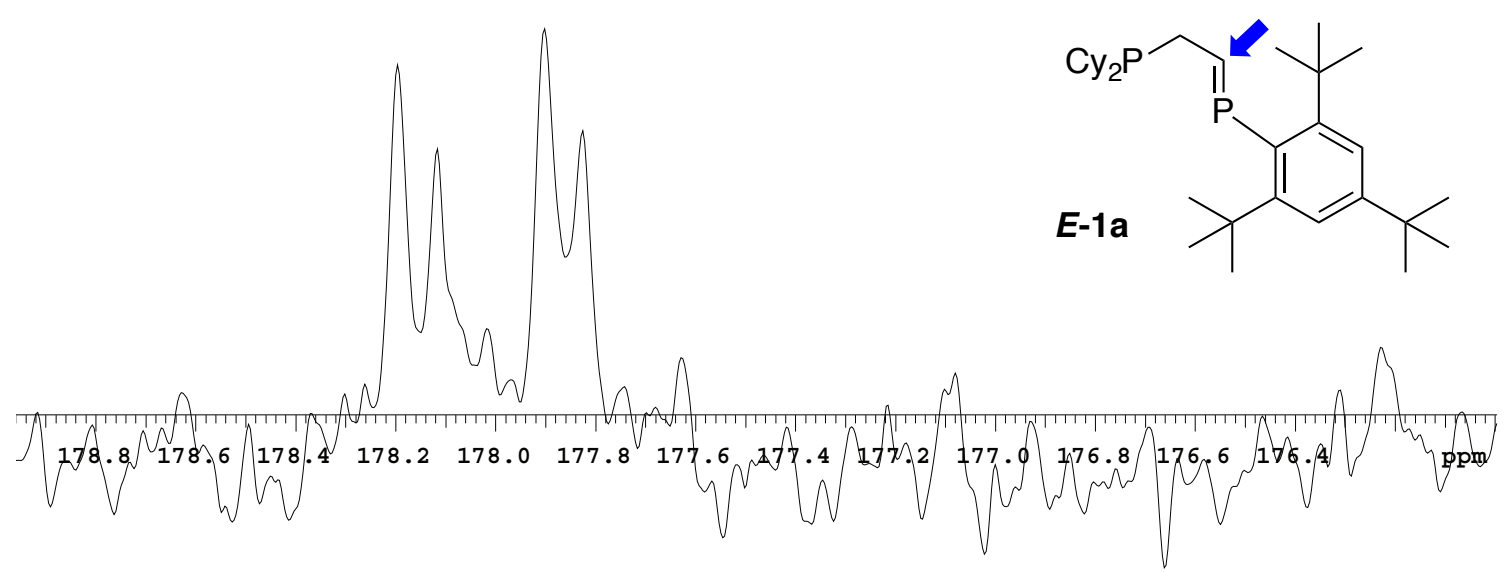

${ }^{13} \mathrm{C}\left\{{ }^{1} \mathrm{H}\right\}$ NMR $\left(\mathrm{C}_{6} \mathrm{D}_{6}\right)$ : Cyclohexyl, backbone $\mathrm{CH}_{2}$, and $t$-Bu region

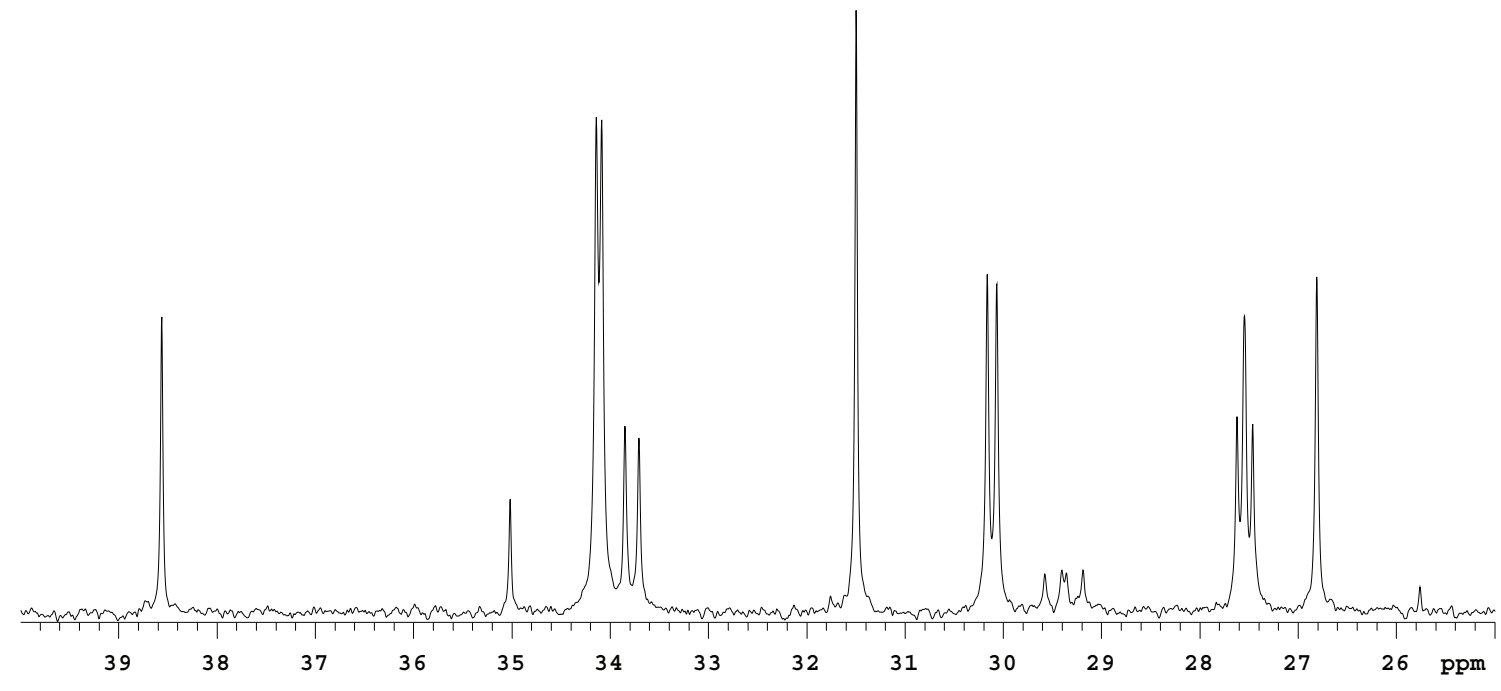




\section{Elemental Analysis for $E$-1a}

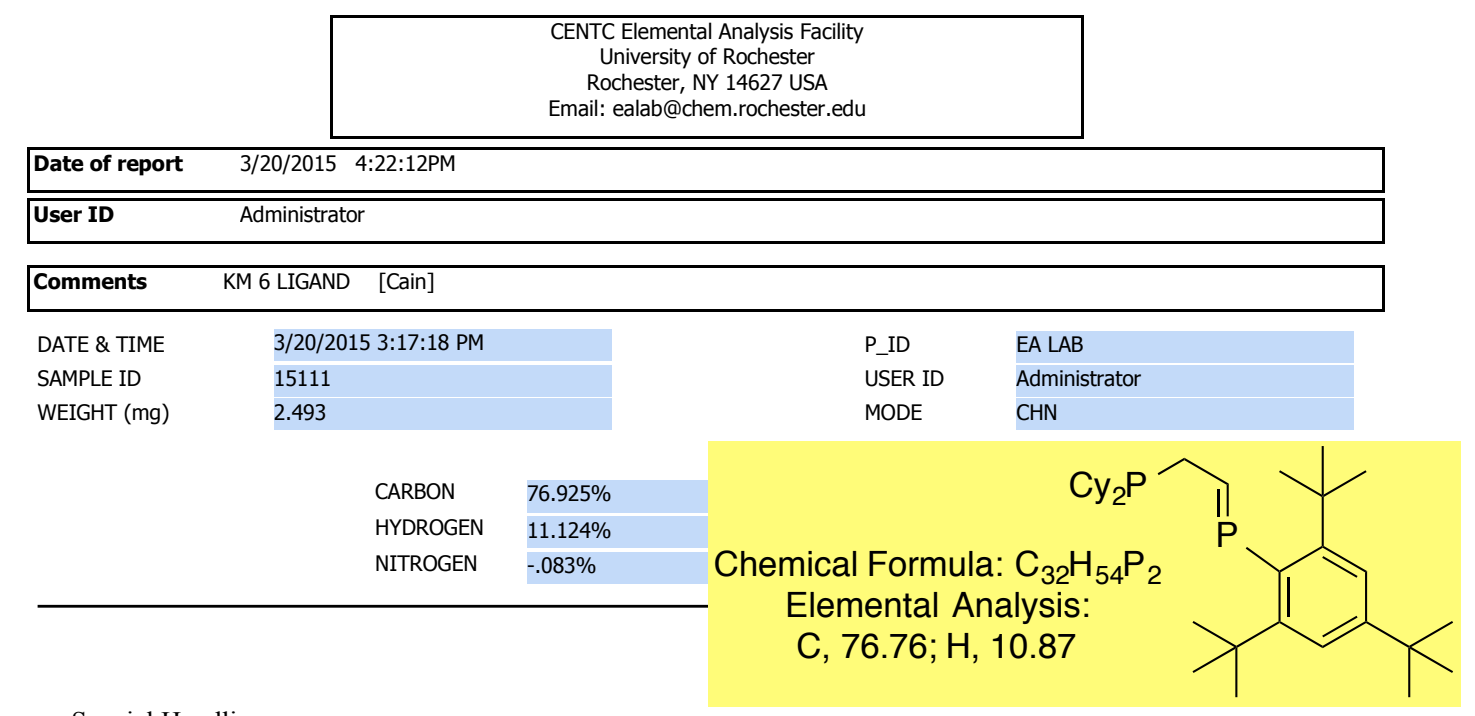

\section{Special Handling}

The sample was transferred under argon and was combusted in a tin capsule that was crimp-sealed with a die apparatus.

Acknowledgment

Analytical data were obtained from the CENTC Elemental Analysis Facility at the University of Rochester, funded by NSF CHE-0650456.

\section{Instrumentation}

Microanalysis samples were weighed with a PerkinElmer Model AD-6 Autobalance and their compositions were determined with a PerkinElmer 2400 Series II Analyzer. Air-sensitive samples were handled in a VAC Atmospheres glovebox. 
Synthesis of $\mathrm{Ph}_{2} \mathrm{P}-\mathrm{CH}_{2}-\mathrm{C}(\mathrm{H})=\mathrm{PMes} *(\mathrm{E}-1 \mathrm{~b})$

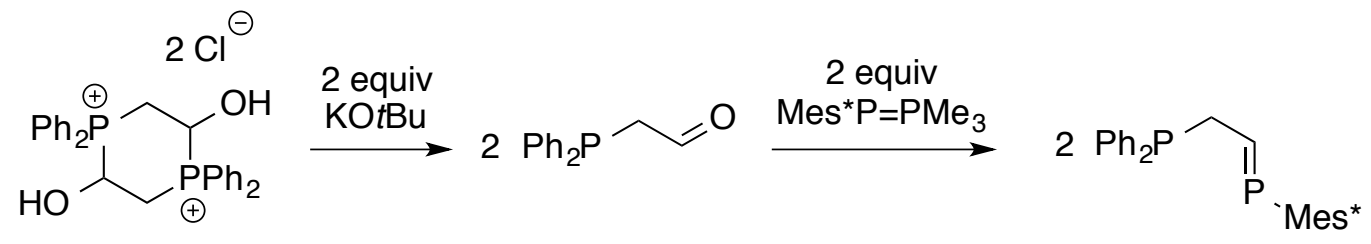

Mes* $\mathrm{PCl}_{2}$ (486 mg, $1.40 \mathrm{mmol}$ ) and $\mathrm{Zn}$ dust $(458 \mathrm{mg}, 7.01 \mathrm{mmol}, 5$ equiv relative to Mes* $\mathrm{PCl}_{2}$ ) were loaded into a vial and $6 \mathrm{~mL}$ of benzene was added. The heterogeneous mixture was cooled to $-35^{\circ} \mathrm{C}$. A solution of $\mathrm{PMe}_{3}(266 \mathrm{mg}, 3.50 \mathrm{mmol}, 2.6$ equiv relative to $\mathrm{Mes}^{*} \mathrm{PCl}_{2}$ ) in $4 \mathrm{~mL}$ of benzene was then added to the frozen mixture. The reaction mixture was warmed to RT and stirred for $2 \mathrm{~h}$. After $2 \mathrm{~h}$, the heterogeneous reaction mixture was filtered through a Celite plug affording a bright yellow filtrate. Concurrently, the phosphonium dimer (370 mg, $0.699 \mathrm{mmol})$ and $\mathrm{KOtBu}(161 \mathrm{mg}, 1.44 \mathrm{mmol}$ ) were combined in a separate vial and $5 \mathrm{~mL}$ of benzene was added. The heterogeneous reaction mixture was vigorously stirred resulting in increased homogeneity and transparency of the solution. After $15 \mathrm{~min}$, the mixture was filtered through a Celite plug into the same vessel as the yellow filtrate. The merged solutions were transferred to a screw-top Teflon bomb and sealed. The homogeneous reaction mixture was stirred inside the glove box overnight $(12 \mathrm{~h})$ before being concentrated under vacuum. (Note: Unlike the Cy derivative, the reaction of the $\mathrm{Ph}$ analogue is rapid and does not require any additional heat). The crude product mixture was dissolved in $20 \mathrm{~mL}$ of pentane and filtered through a Celite plug to remove the $\mathrm{PMe}_{3}(0)$. The yellow filtrate was then concentrated under vacuum affording a sticky solid. The crude product was dissolved in $2 \mathrm{~mL}$ of THF and layered with $8 \mathrm{~mL}$ of acetonitrile, resulting in the precipitation of a crystalline yellow solid $(402 \mathrm{mg}, 0.823 \mathrm{mmol}$, $59 \%$ ). X-ray quality crystals were obtained by dissolving a $20 \mathrm{mg}$ sample of ligand in $4 \mathrm{~mL}$ of a 50:50 solution of THF and acetonitrile at $-35^{\circ} \mathrm{C}$.

Anal. Calcd. for $\mathrm{C}_{32} \mathrm{H}_{42} \mathrm{P}_{2}$ : C, 78.66; $\mathrm{H}, 8.66$. Found: C, 78.76; H, 8.80. HRMS $m / z$ calcd for $\mathrm{C}_{32} \mathrm{H}_{42} \mathrm{P}_{2}\left(\mathrm{MH}^{+}\right): 488.2762$. Found: 488.2782. ${ }^{31} \mathrm{P}\left\{{ }^{1} \mathrm{H}\right\} \mathrm{NMR}\left(\mathrm{CDCl}_{3}\right): \delta 259.2(\mathrm{~d}, J=47$, $\mathrm{P}=\mathrm{C}$ ), $-16.3\left(\mathrm{~d}, J=47, \mathrm{PPh}_{2}\right) .{ }^{1} \mathrm{H}$ NMR $\left(\mathrm{CDCl}_{3}\right): \delta 7.49(\mathrm{~m}, 4 \mathrm{H}, \mathrm{Ph}), 7.37-7.32$ (overlapping $\mathrm{m}$, 9H, $\mathrm{P}=\mathrm{CH}, \mathrm{Ph}$, and Mes*), 3.47 (ddd, $J=2.5,9,21,2 \mathrm{H}, \mathrm{CH}_{2}$ backbone), $1.39(18 \mathrm{H}, t-\mathrm{Bu}), 1.33$ (9H, $t$-Bu). ${ }^{13} \mathrm{C}\left\{{ }^{1} \mathrm{H}\right\}$ NMR $\left(\mathrm{CDCl}_{3}\right): \delta 172.0$ (dd, $\left.J=36,8, \mathrm{P}=\mathrm{C}\right), 153.6(\mathrm{Ar}), 149.3(\mathrm{Ar}), 138.8$ (d, $J=57, A r), 137.9(\mathrm{~d}, J=15, \mathrm{Ph}), 132.9(\mathrm{~d}, J=18, \mathrm{Ph}), 128.7(\mathrm{Ph})$ and $128.4(\mathrm{~d}, J=7, \mathrm{Ph}$ ), 121.6 (Ar), $38.0\left(\mathrm{CMe}_{3}\right), 35.5$ (dd, $J=28,13$, backbone $\left.\mathrm{CH}_{2}\right), 34.9\left(\mathrm{CMe}_{3}\right), 33.6(\mathrm{~d}, J=7, t-$ $\mathrm{Bu}), 31.3(t-\mathrm{Bu})$. 
NMR Spectroscopy of $E-1 b$

${ }^{31} \mathrm{P}\left\{{ }^{1} \mathrm{H}\right\} \mathrm{NMR}\left(\mathrm{CDCl}_{3}\right)$

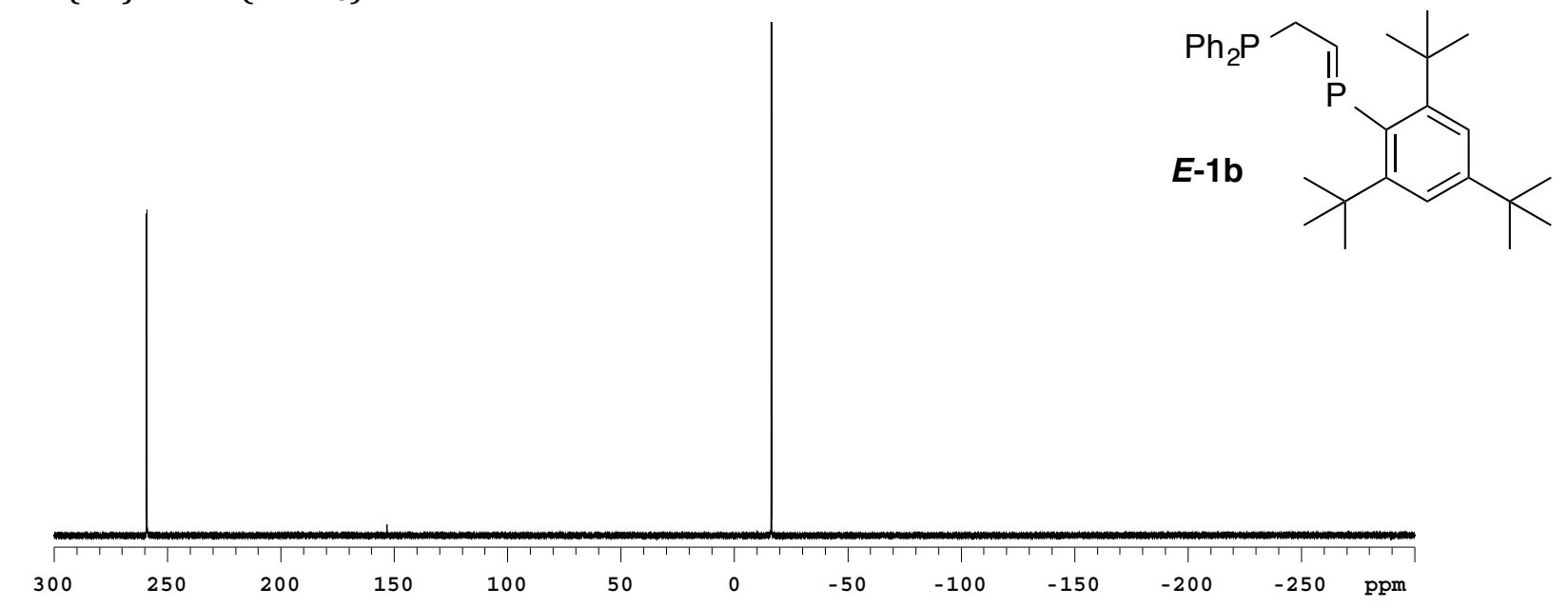

${ }^{31} \mathrm{P}\left\{{ }^{1} \mathrm{H}\right\}$ NMR $\left(\mathrm{CDCl}_{3}\right)$ : Selected Zoom

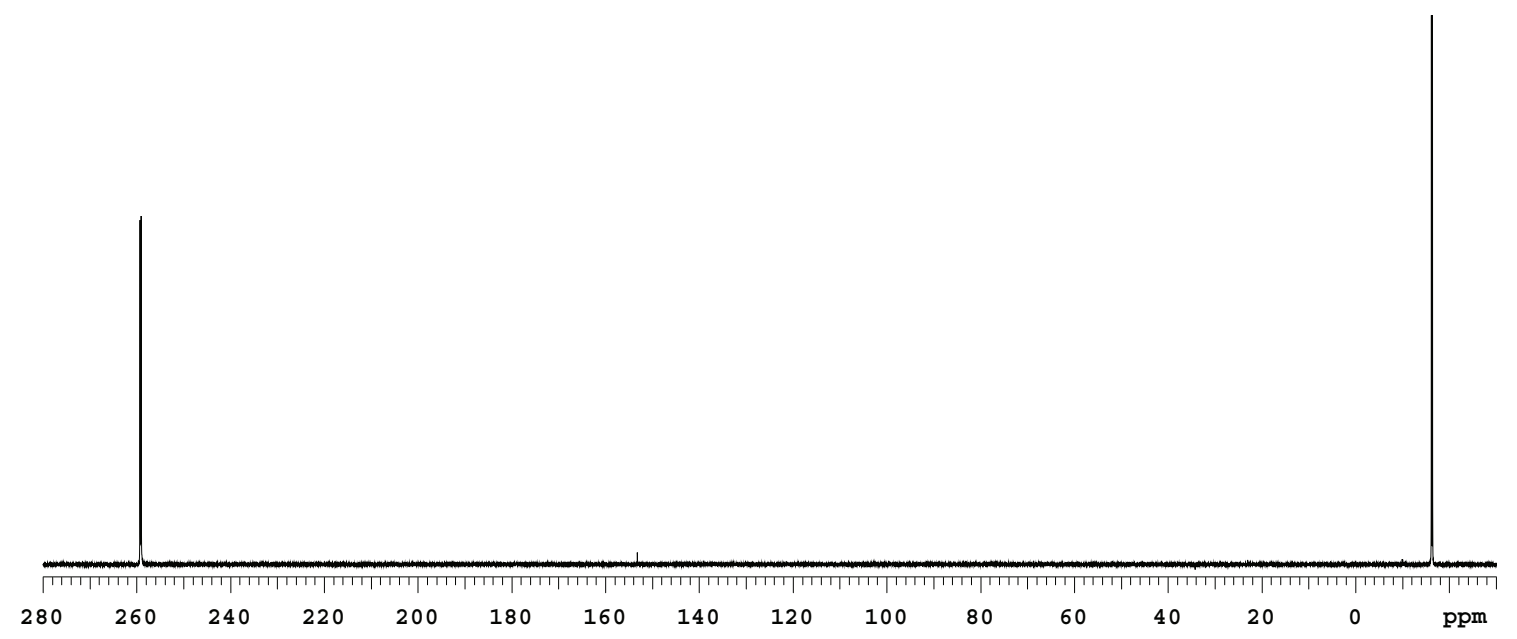


${ }^{1} \mathrm{H} \mathrm{NMR}\left(\mathrm{CDCl}_{3}\right)$

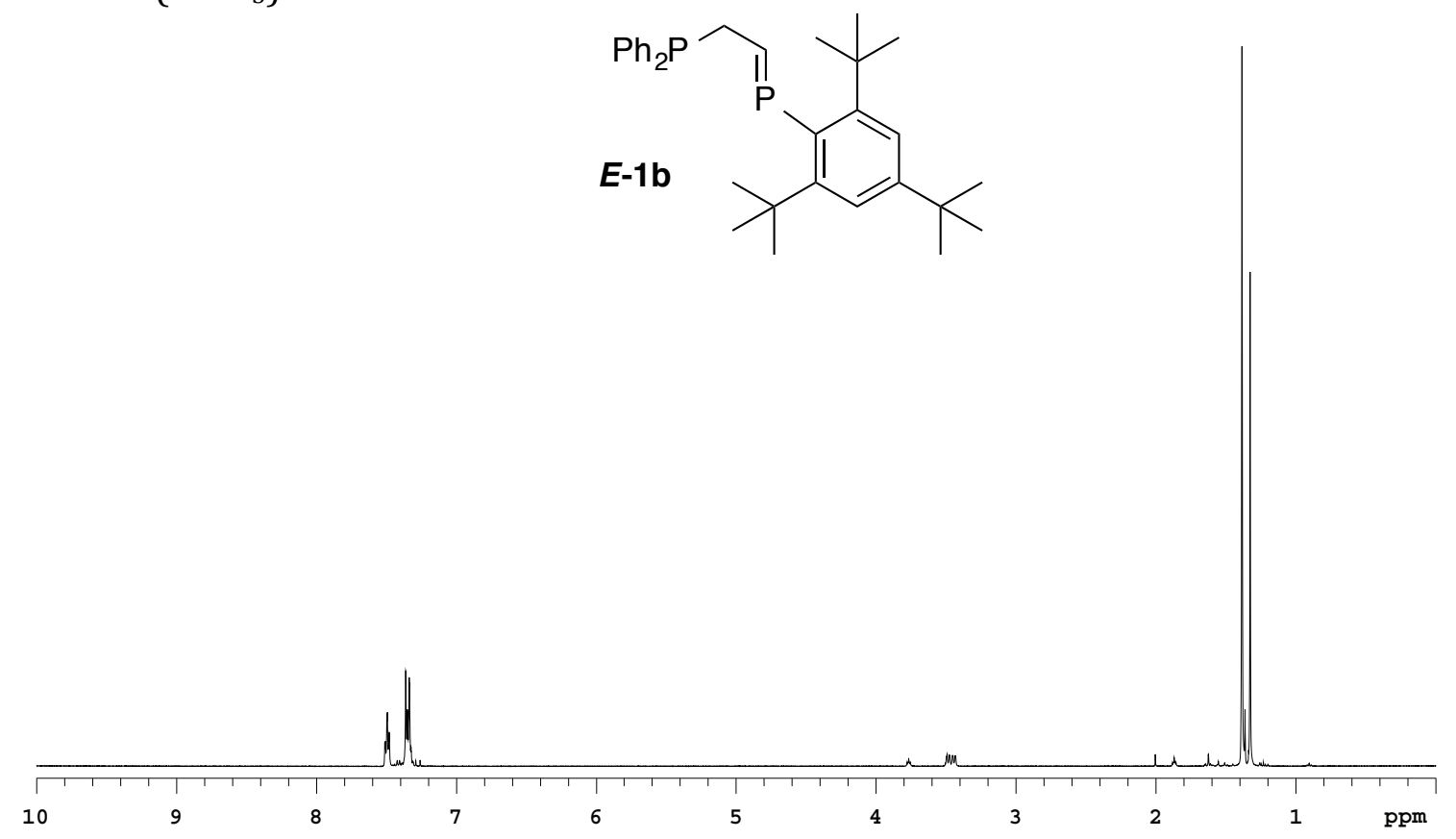

${ }^{1} \mathrm{H}$ NMR $\left(\mathrm{CDCl}_{3}\right):$ Selected Zoom (trace ether present)

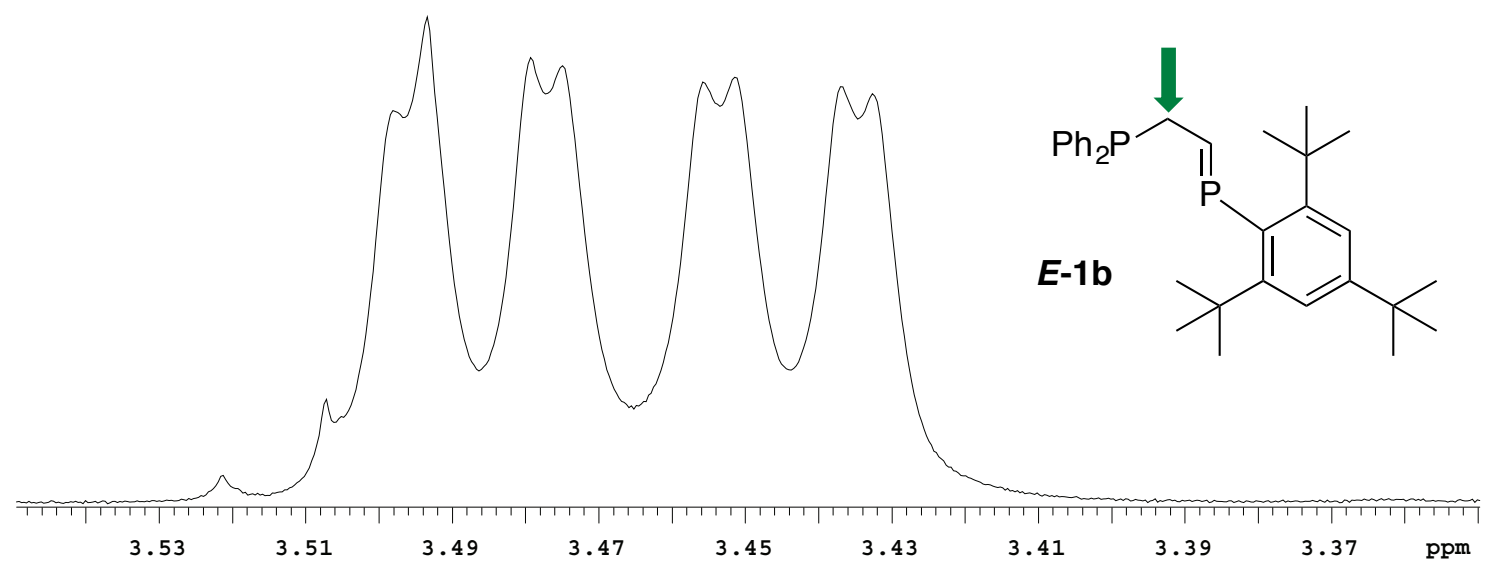


${ }^{13} \mathrm{C}\left\{{ }^{1} \mathrm{H}\right\}$ NMR $\left(\mathrm{CDCl}_{3}\right)$

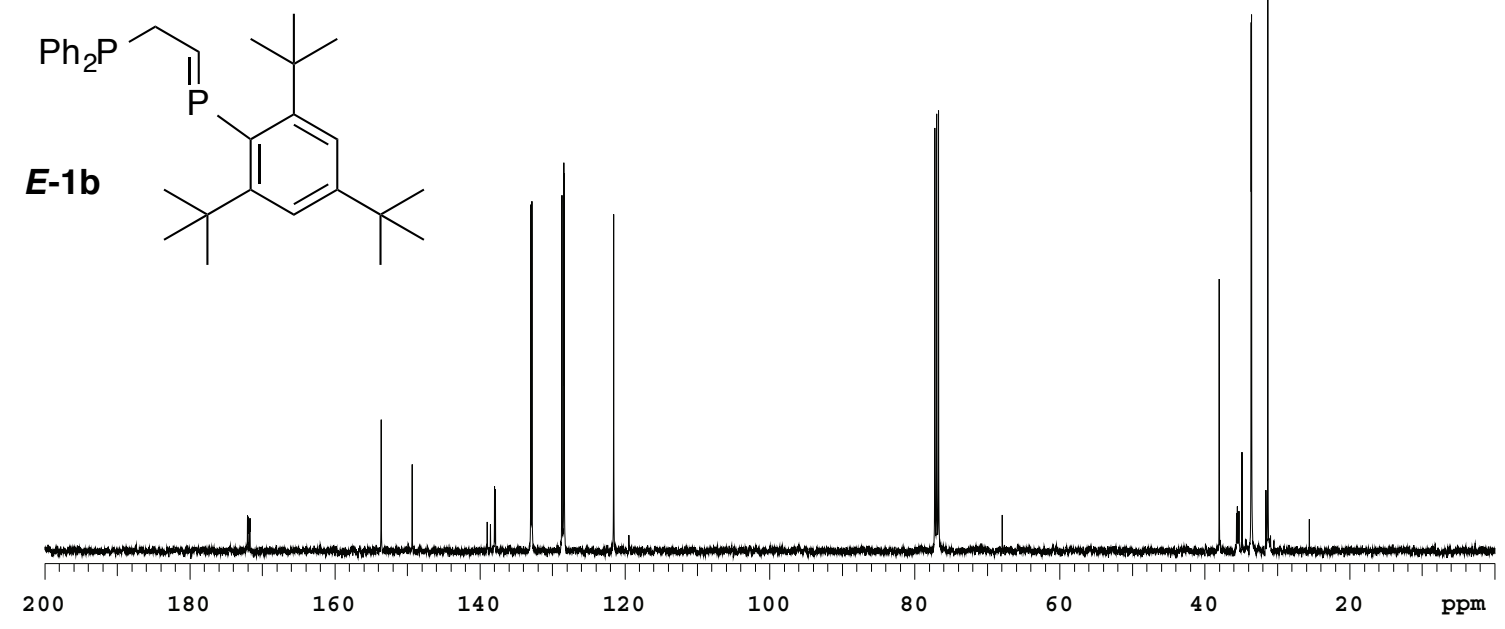

${ }^{13} \mathrm{C}\left\{{ }^{1} \mathrm{H}\right\}$ NMR $\left(\mathrm{CDCl}_{3}\right)$ : Selected Zoom

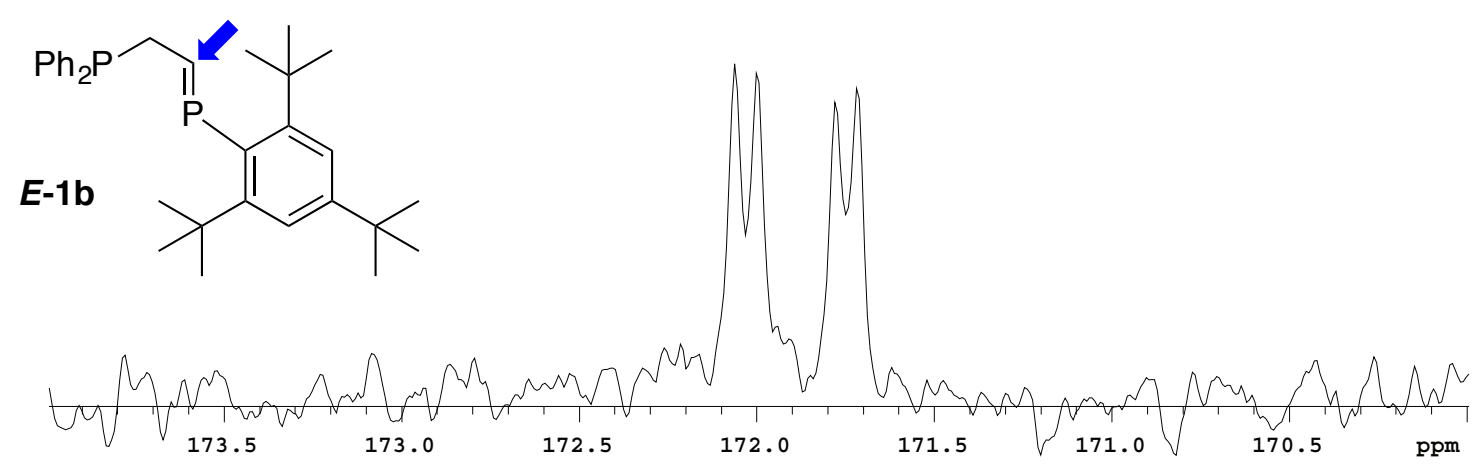




\section{Elemental Analysis for $E-1 b$}

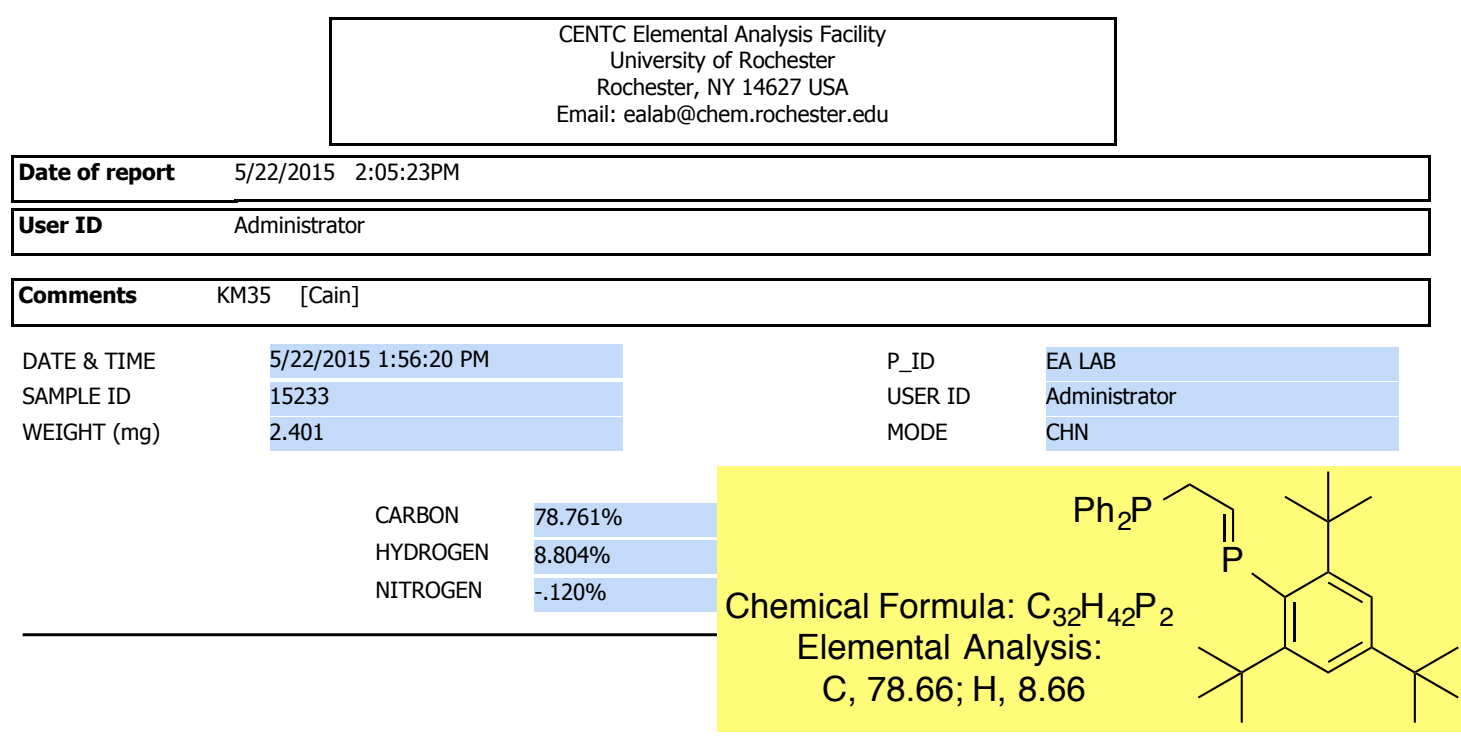

Special Handling

The sample was transferred under argon and was combusted in a tin capsule that was crimp-sealed with a die apparatus.

\section{$\underline{\text { Acknowledgment }}$}

Analytical data were obtained from the CENTC Elemental Analysis Facility at the University of Rochester, funded by NSF CHE-0650456.

\section{Instrumentation}

Microanalysis samples were weighed with a PerkinElmer Model AD-6 Autobalance and their compositions were determined with a PerkinElmer 2400 Series II Analyzer. Air-sensitive samples were handled in a VAC

Atmospheres glovebox. 
Synthesis of Pd and Pt complexes of E-1a:

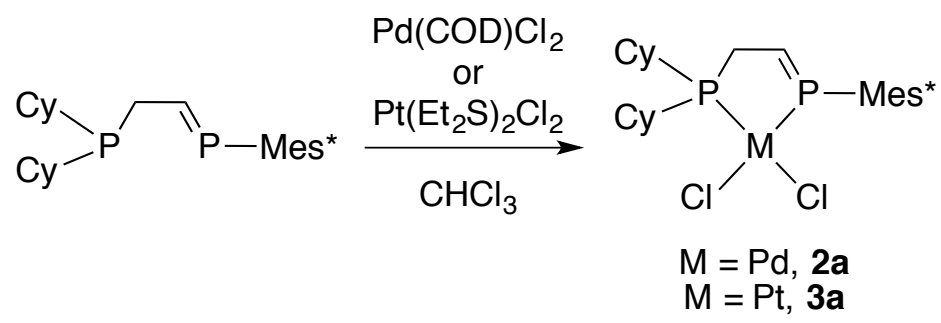

\section{Pd complex (2a)}

Treatment of $\mathrm{Pd}(\mathrm{COD}) \mathrm{Cl}_{2}(57 \mathrm{mg}, 0.200 \mathrm{mmol})$ with a solution of $1 \mathrm{a}(100 \mathrm{mg}, 0.200 \mathrm{mmol})$ in $3 \mathrm{~mL}$ of chloroform resulted in a homogeneous yellow-orange reaction mixture. After 30 min of stirring, ${ }^{31} \mathrm{P}$ NMR spectroscopy $\left(\mathrm{CHCl}_{3}\right)$ revealed metallation was complete $(\delta 231$ and $103 \mathrm{ppm}$ ), and the solution was subsequently filtered through a Celite plug. The filtrate was layered with approximately $12 \mathrm{~mL}$ of pentane and placed in the glove box freezer at $-35{ }^{\circ} \mathrm{C}$, affording a pale yellow powder (114 mg, $\left.0.168 \mathrm{mmol}, 84 \%\right)$. X-ray quality crystals were obtained serendipitously. While monitoring the air-sensitivity of 2a over the course of two days, the homogeneous solution $\left(\mathrm{CDCl}_{3}\right)$ evaporated slowly and deposited pale yellow crystals.

Elemental analysis data was consistently low in carbon (three trials), for example: Anal. Calcd. for $\mathrm{C}_{32} \mathrm{H}_{54} \mathrm{P}_{2} \mathrm{PdCl}_{2}$ : C, 56.68; H, 8.03. Found: C, 55.94; H, 8.15. HRMS $m / z$ calcd for $\mathrm{C}_{32} \mathrm{H}_{54} \mathrm{P}_{2} \mathrm{PdCl}(\mathrm{M}-\mathrm{Cl})^{+}:$641.2424. Found: 641.2443. ${ }^{31} \mathrm{P}\left\{{ }^{1} \mathrm{H}\right\} \mathrm{NMR}\left(\mathrm{CDCl}_{3}\right): \delta 230.6(\mathrm{~d}, J=$ 17, $\mathrm{P}=\mathrm{C}), 103.1(\mathrm{~d}, J=17, \mathrm{PCy} 2) .{ }^{1} \mathrm{H}$ NMR $\left(\mathrm{CDCl}_{3}\right): \delta 7.50\left(\mathrm{~d}, J=4,2 \mathrm{H}, \mathrm{Mes}^{*}\right), 7.00$ (ddt, $J=4$, 13.5, 52, $1 \mathrm{H}, \mathrm{P}=\mathrm{CH}$ ), 2.57-2.42 (overlapping multiplets, $4 \mathrm{H}, \mathrm{Cy}$ and $\mathrm{CH}_{2}$ backbone), 2.27 (br, 2H, Cy), 1.85 (br m, 6H, Cy), 1.74 (br m, 4H, Cy), 1.65 (18H, $t-\mathrm{Bu}), 1.57$ (m, 2H, Cy), 1.40$1.26(6 \mathrm{H}, \mathrm{Cy}), 1.31(9 \mathrm{H}, t-\mathrm{Bu}) .{ }^{13} \mathrm{C}\left\{{ }^{1} \mathrm{H}\right\}$ NMR $\left(\mathrm{CDCl}_{3}\right): \delta 159.8(\mathrm{dd}, J=63,17, \mathrm{P}=\mathrm{C}), 155.7$ (Ar), 154.2 (Ar), 123.7 (d, $J=10, A r), 122.8$ (d, $J=16$, Ar), $39.1\left(\mathrm{CMe}_{3}\right), 36.2$ (d, $J=27$, P$\mathrm{CH}(\mathrm{Cy})$ ), $35.3\left(\mathrm{CMe}_{3}\right), 34.8(t-\mathrm{Bu}), 31.0(t-\mathrm{Bu}), 28.3(\mathrm{~d}, J=38$, Cy), 27.1 (overlapping dd, $J=$ 28, 25, $\mathrm{CH}_{2}$ backbone), 26.5 (m, Cy), $25.7(\mathrm{Cy}) \cdot{ }^{13} \mathrm{C}\left\{{ }^{1} \mathrm{H}\right\}$ NMR assignments were aided by DEPT experiments. 


\section{NMR Spectroscopy for 2a}

${ }^{31} \mathrm{P}\left\{{ }^{1} \mathrm{H}\right\} \mathrm{NMR}\left(\mathrm{CDCl}_{3}\right)$

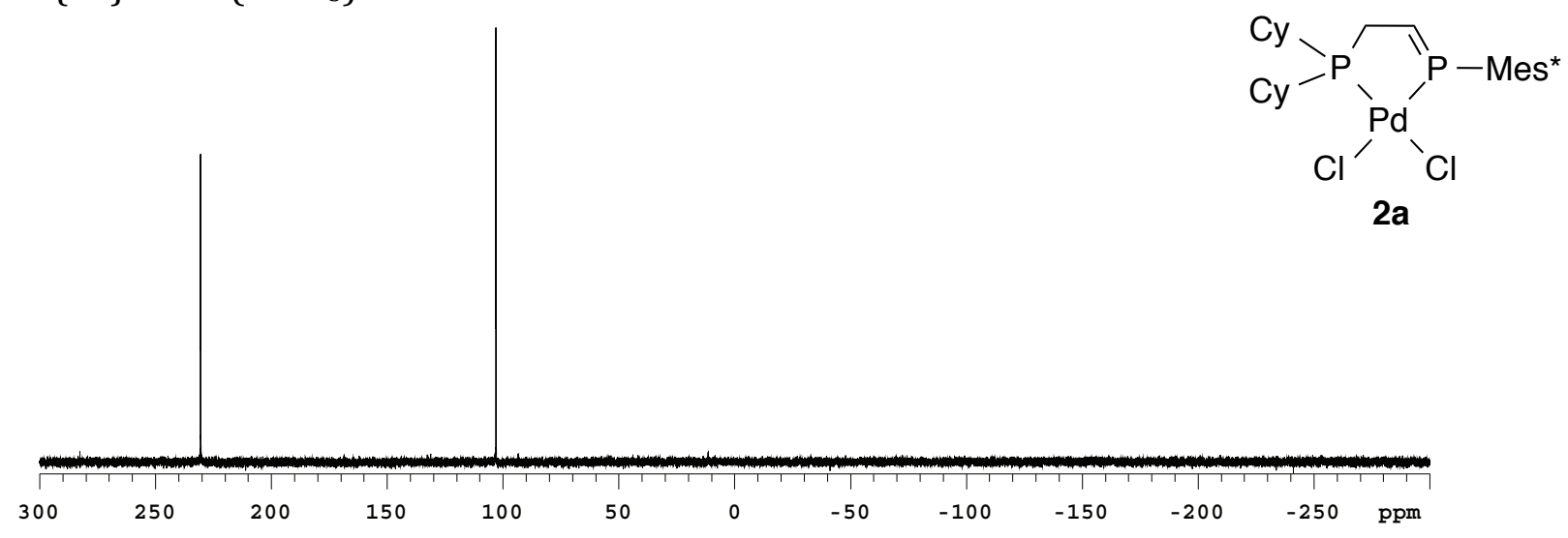

${ }^{31} \mathrm{P}\left\{{ }^{1} \mathrm{H}\right\}$ NMR $\left(\mathrm{CDCl}_{3}\right):$ Selected Zoom

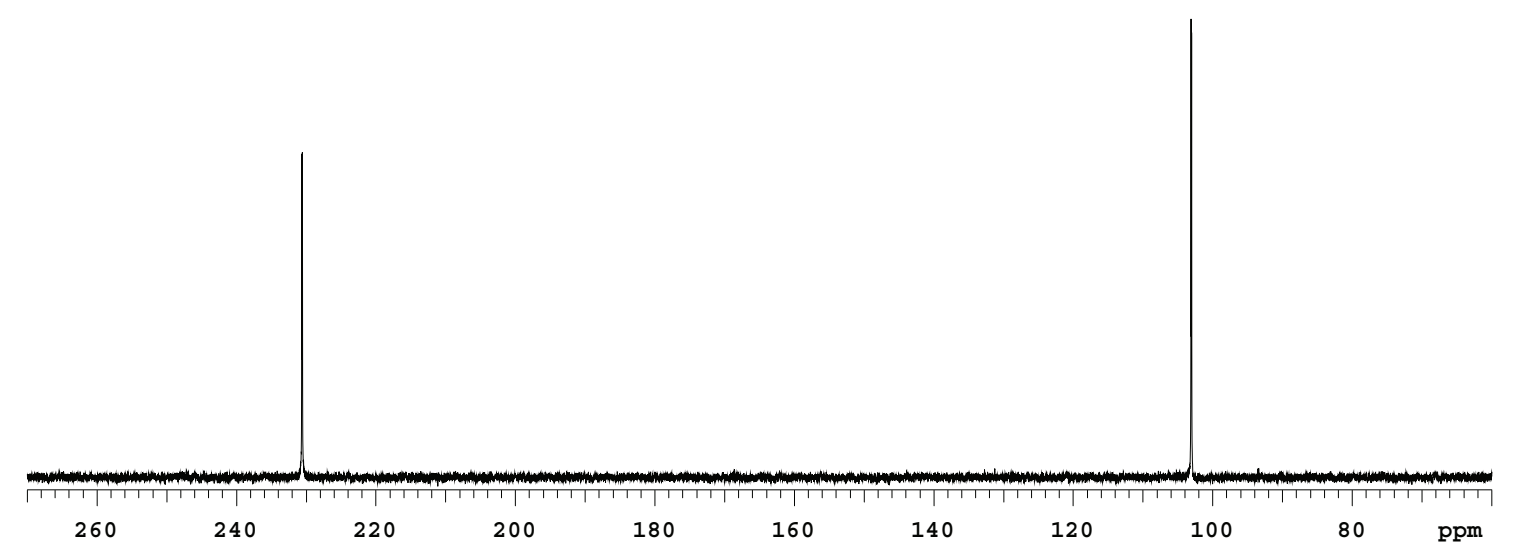


${ }^{1} \mathrm{H} \mathrm{NMR}\left(\mathrm{CDCl}_{3}\right)$
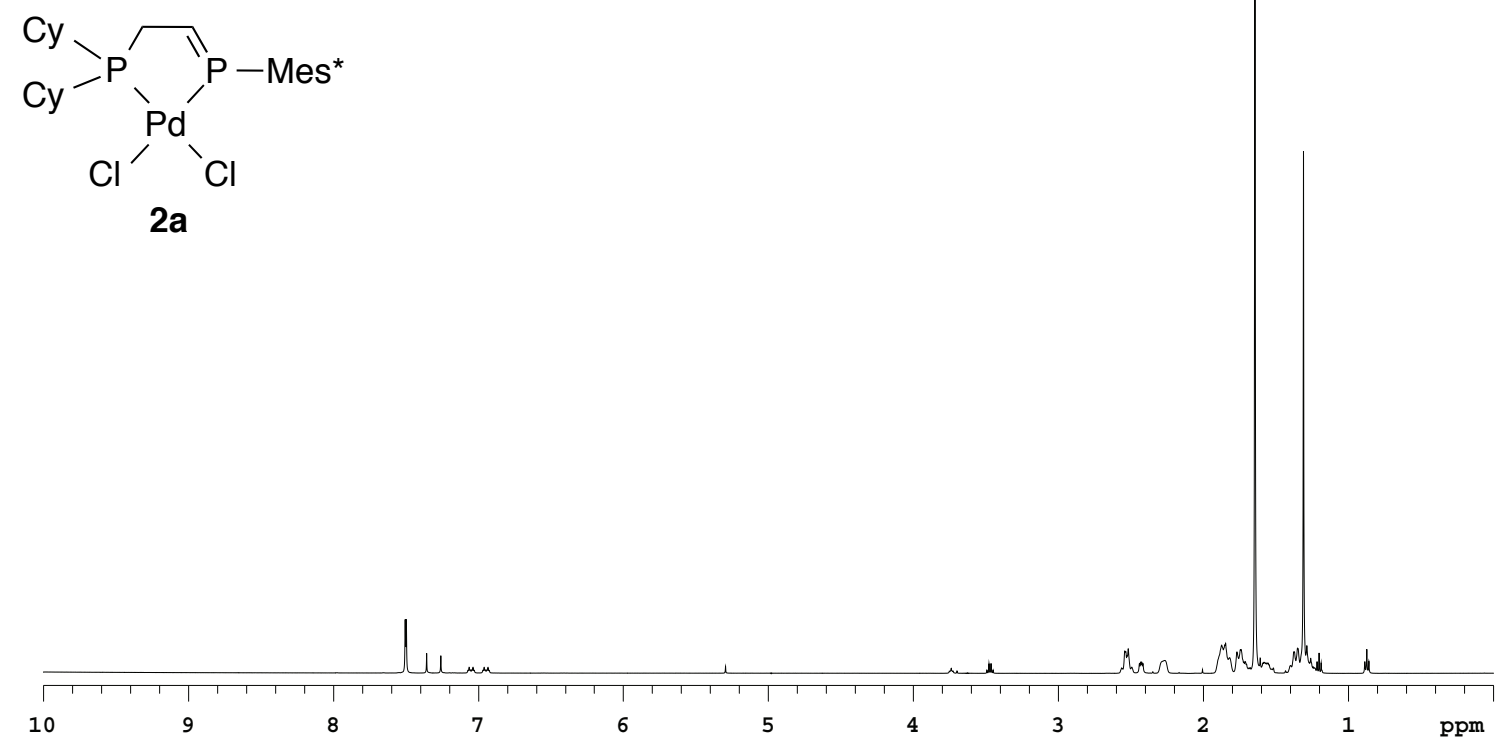

${ }^{1} \mathrm{H}$ NMR $\left(\mathrm{CDCl}_{3}\right)$ : Selected Zoom

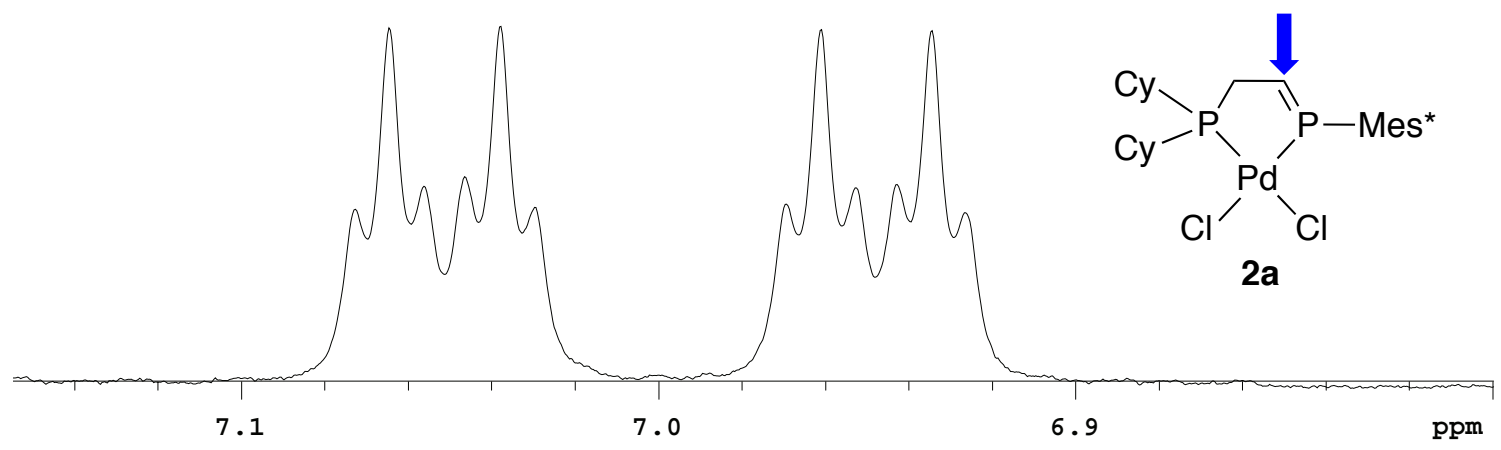


${ }^{13} \mathrm{C}\left\{{ }^{1} \mathrm{H}\right\} \mathrm{NMR}\left(\mathrm{CDCl}_{3}\right)$

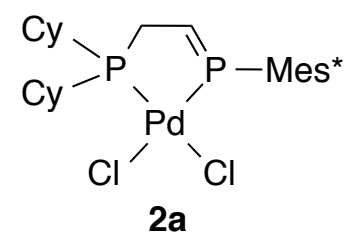

${ }^{13} \mathrm{C}\left\{{ }^{1} \mathrm{H}\right\}$ NMR $\left(\mathrm{CDCl}_{3}\right)$ : Selected Zoom

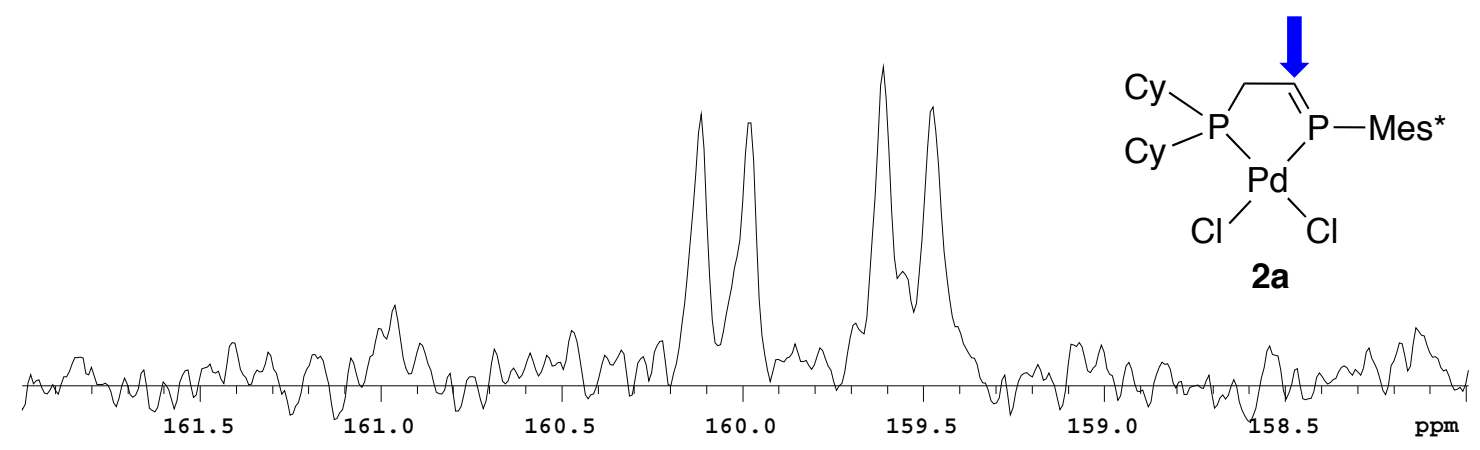


${ }^{13} \mathrm{C}\left\{{ }^{1} \mathrm{H}\right\}$ NMR $\left(\mathrm{CDCl}_{3}\right)$ : Cyclohexyl, backbone $\mathrm{CH}_{2}$, and $t$-Bu region

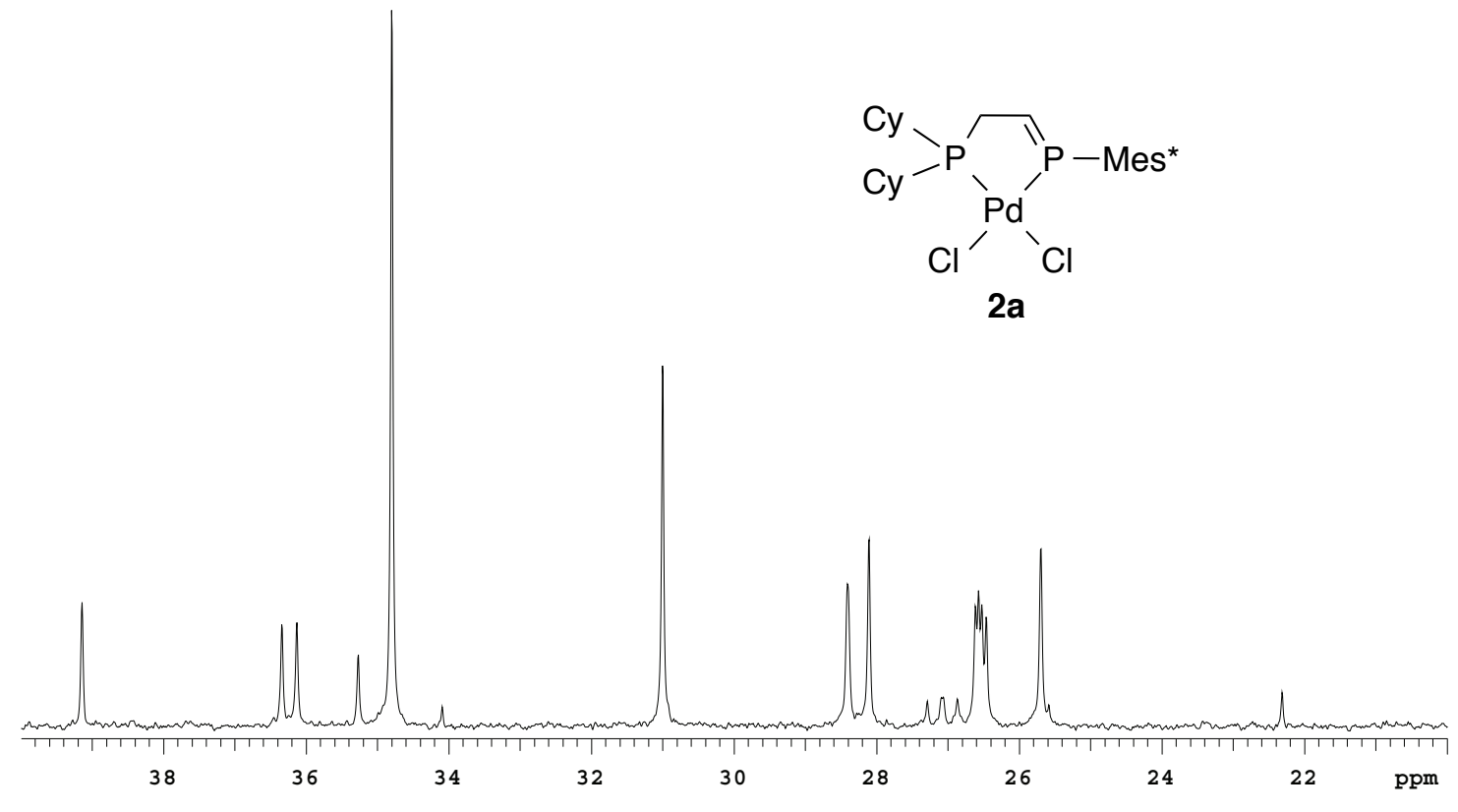




\section{Elemental Analysis for 2a}

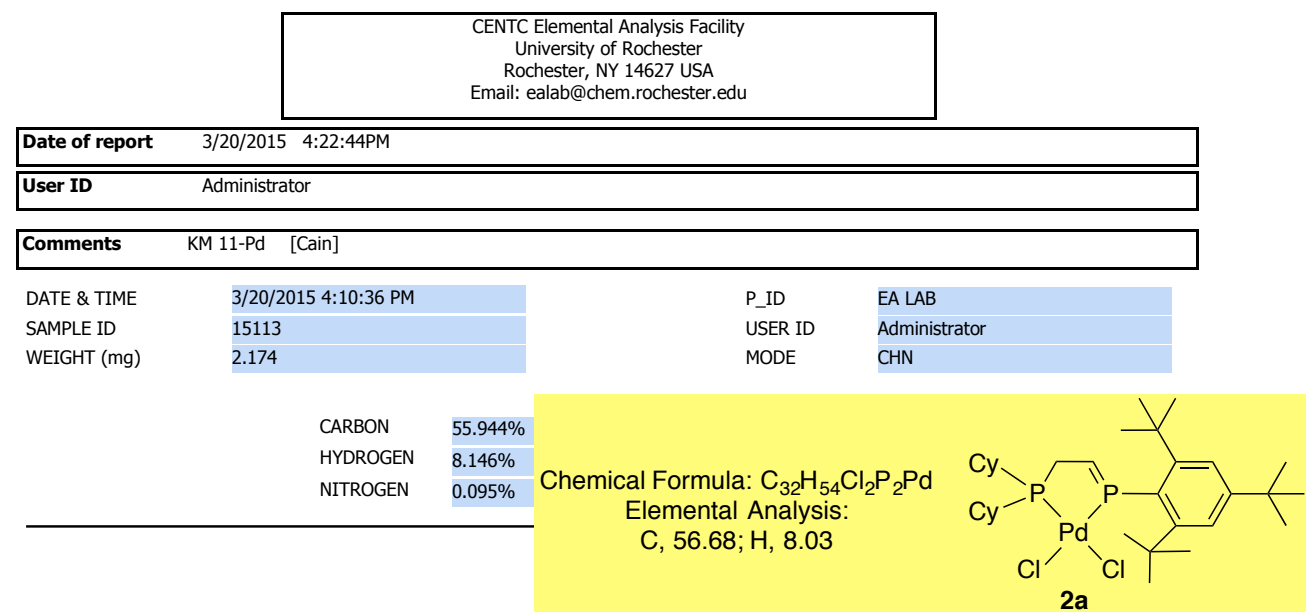

Special Handling

The sample was transferred under argon and was combusted in a tin capsule that was crimp-sealed with a die apparatus.

$\underline{\text { Acknowledgment }}$

Analytical data were obtained from the CENTC Elemental Analysis Facility at the University of Rochester, funded by NSF CHE-0650456.

$\underline{\text { Instrumentation }}$

Microanalysis samples were weighed with a PerkinElmer Model AD-6 Autobalance and their compositions were determined with a PerkinElmer 2400 Series II Analyzer. Air-sensitive samples were handled in a VAC Atmospheres glovebox 


\section{Pt complex (3a)}

Treatment of $\mathrm{Pt}\left(\mathrm{Et}_{2} \mathrm{~S}\right)_{2} \mathrm{Cl}_{2}(45 \mathrm{mg}, 0.100 \mathrm{mmol})$ with a solution of $1 \mathrm{a}(50 \mathrm{mg}, 0.100 \mathrm{mmol})$ in $2 \mathrm{~mL}$ of chloroform resulted in a homogeneous yellow-orange reaction mixture, which within a minute started to deposit an off white solid. Stirring was continued for 30 minutes before the reaction mixture was layered with approximately $12 \mathrm{~mL}$ of pentane and placed in the glove box freezer at $-35{ }^{\circ} \mathrm{C}$ to enhance the precipitation of the off white powder (66 $\mathrm{mg}, 0.086 \mathrm{mmol}, 86 \%$ ).

The solubility of 3a was problematic. A sufficient ${ }^{31} \mathrm{P}\left\{{ }^{1} \mathrm{H}\right\}$ NMR spectrum could be obtained in chloroform, but the corresponding ${ }^{1} \mathrm{H}$ and ${ }^{13} \mathrm{C}\left\{{ }^{1} \mathrm{H}\right\}$ NMR displayed a poor signal to noise ratio. The complex (3a) is also slightly soluble in $\mathrm{CD}_{3} \mathrm{CN}$, but at higher temperatures, the reaction mixture maintains its homogeneity and improved NMR data can be obtained. Xray quality crystals were obtained after the NMR sample in $\mathrm{CD}_{3} \mathrm{CN}$ at $70{ }^{\circ} \mathrm{C}$ cooled to $\mathrm{RT}$ and deposited translucent crystals.

Elemental analysis data was consistently low in carbon (three trials), for example: Anal. Calcd. for $\mathrm{C}_{32} \mathrm{H}_{54} \mathrm{P}_{2} \mathrm{PtCl}_{2}$ : C, 50.13; H, 7.10. Found: C, 49.27; H, 6.87. HRMS $\mathrm{m} / z$ calcd for $\mathrm{C}_{32} \mathrm{H}_{55} \mathrm{P}_{2} \mathrm{PtCl}_{2} \mathrm{Na}(\mathrm{MH}+\mathrm{Na})^{+}:$789.2701. Found: 789.2637. ${ }^{31} \mathrm{P}\left\{{ }^{1} \mathrm{H}\right\} \mathrm{NMR}\left(\mathrm{CDCl}_{3}\right): \delta 213.3$ $\left(\mathrm{UPtP}_{\mathrm{Pt}}=4520, \mathrm{P}=\mathrm{C}\right), 68.4\left(\mathrm{JPtP}_{\mathrm{PtP}}=3280, \mathrm{PC} \mathrm{y}_{2}\right)$. On a $300 \mathrm{MHz}$ NMR spectrometer $J_{\mathrm{PP}}$ coupling is observed: JPP $=3 .{ }^{1} \mathrm{H} \mathrm{NMR}\left(\mathrm{CD}_{3} \mathrm{CN}, 70{ }^{\circ} \mathrm{C}\right): \delta 7.66\left(\mathrm{br}, 2 \mathrm{H}, \mathrm{Mes}^{*}\right), 7.22(\mathrm{br} \mathrm{m}, 1 \mathrm{H}, \mathrm{P}=\mathrm{CH}), 2.49$ (broad quartet, $J=11.5,2 \mathrm{H}, \mathrm{Cy}$ ), 2.25-2.13 (overlapping multiplet, $4 \mathrm{H}, \mathrm{Cy}$ and $\mathrm{CH}_{2}$ backbone), 1.86 (br m, 8H, Cy), 1.75-1.27 (broad and overlapping, 10H, Cy), 1.69 (18H, $t$ $\mathrm{Bu}), 1.36(9 \mathrm{H}, t-\mathrm{Bu}) .{ }^{13} \mathrm{C}\left\{{ }^{1} \mathrm{H}\right\}$ NMR $\left(\mathrm{CD}_{3} \mathrm{CN}, 70{ }^{\circ} \mathrm{C}\right): \delta 157.5(\mathrm{Ar}), 156.6(\mathrm{dd}, J=86,10, \mathrm{P}=\mathrm{C})$, 155.7 ( $\mathrm{Ar}$ ), 125.2 (d, $J=11, \mathrm{Ar}), 122.7$ (d, $J=34, \mathrm{Ar}), 40.3\left(\mathrm{CMe}_{3}\right), 36.3(\mathrm{CMe}), 35.9$ (m, P$\mathrm{CH}(\mathrm{Cy})$ ), $35.2(t-\mathrm{Bu}), 31.4(t-\mathrm{Bu}), 29.1(\mathrm{Cy}), 27.4$ (overlapping $\mathrm{m}, \mathrm{CH}_{2}$ backbone and $\mathrm{Cy}$ ), $26.9(\mathrm{Cy}) .{ }^{13} \mathrm{C}$ NMR assignments were aided by DEPT experiments. 

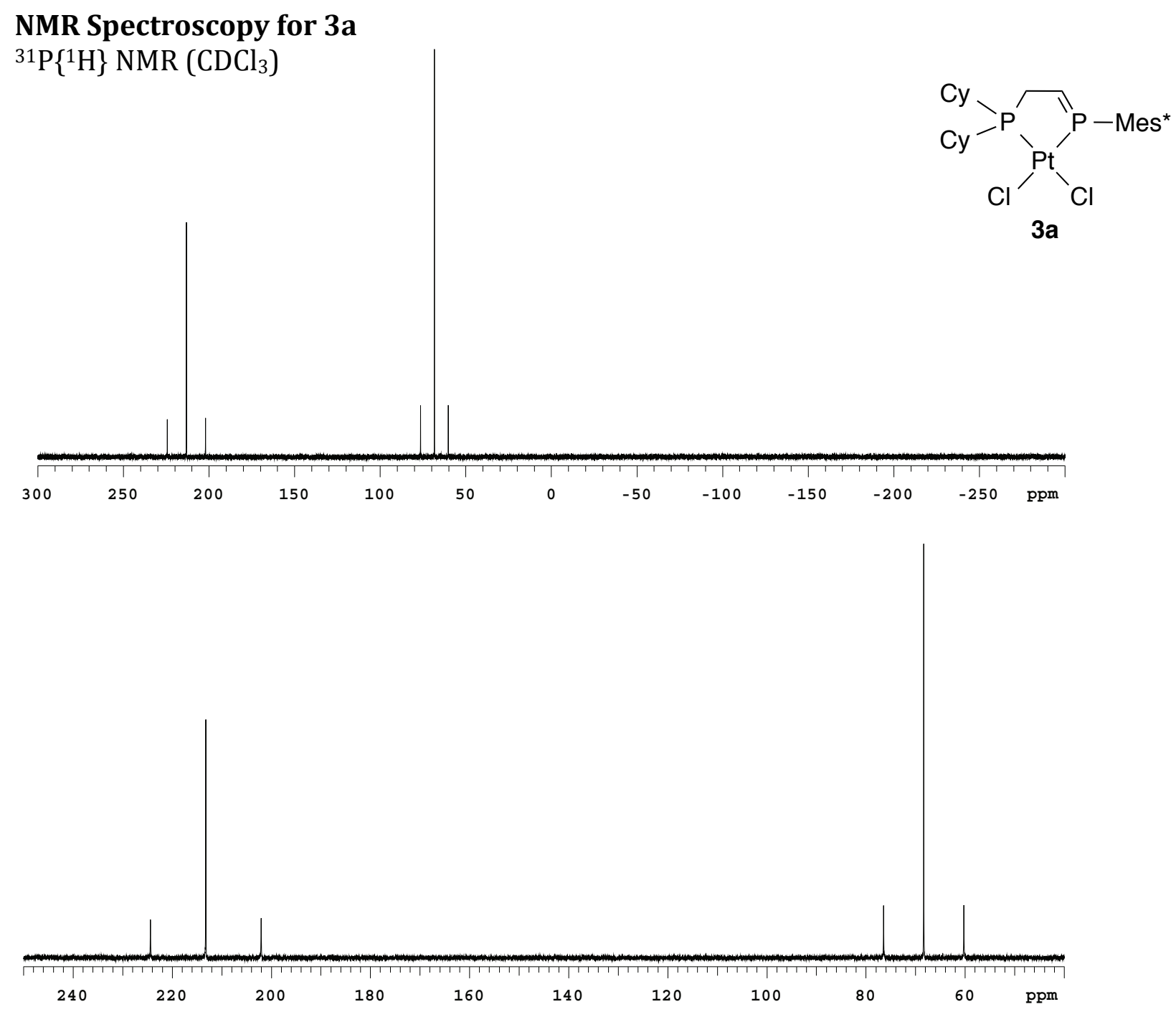
${ }^{1} \mathrm{H}$ NMR $\left(\mathrm{CD}_{3} \mathrm{CN}, 70{ }^{\circ} \mathrm{C}\right)$
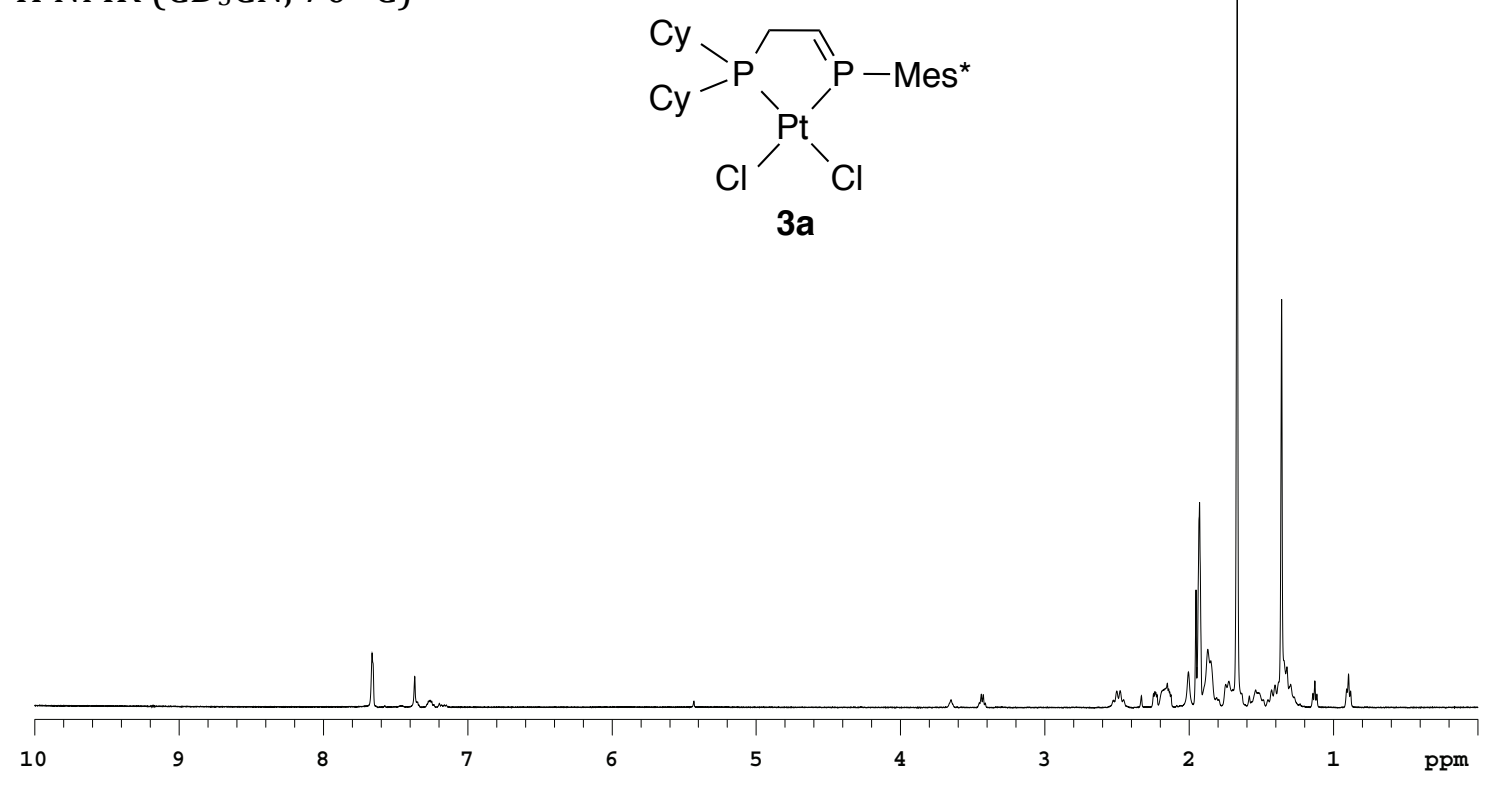


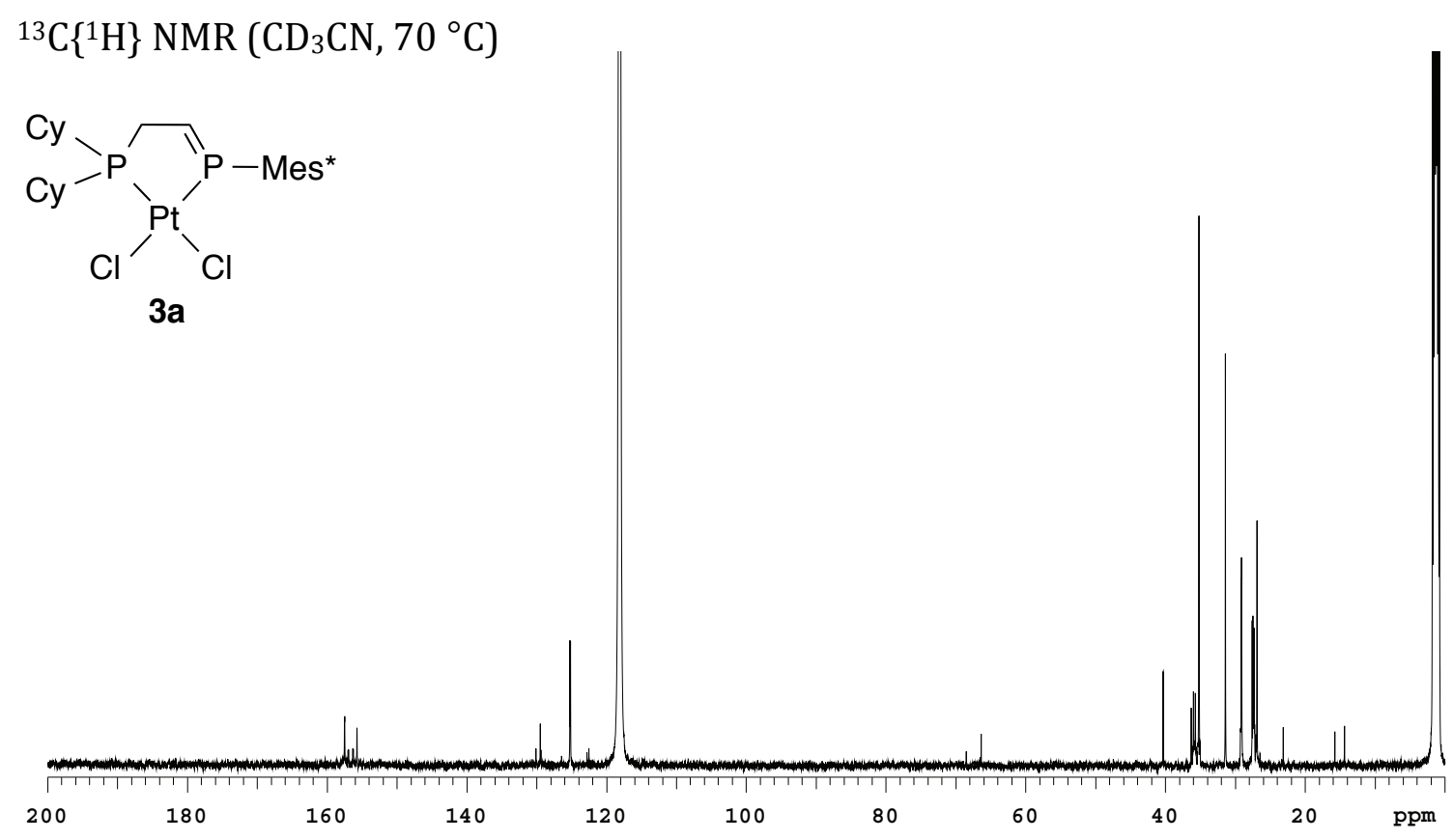

${ }^{13} \mathrm{C}\left\{{ }^{1} \mathrm{H}\right\}$ NMR (CDCN, $\left.70{ }^{\circ} \mathrm{C}\right)$ : Phosphaalkene and Mes* region

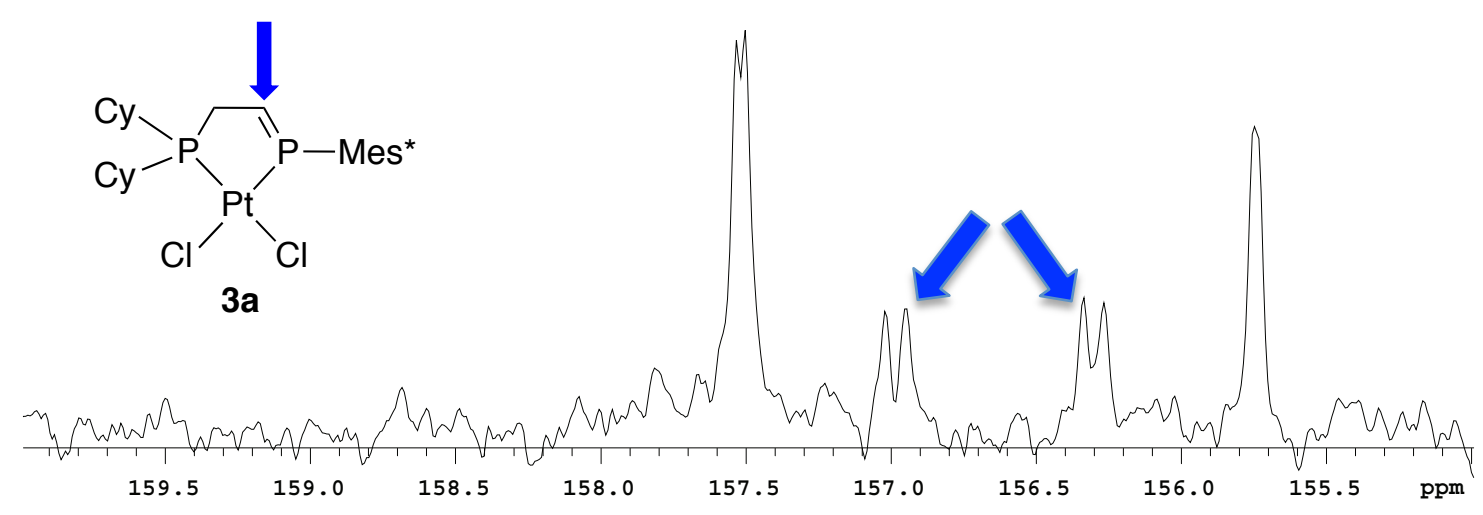




\section{Elemental Analysis for 3a}

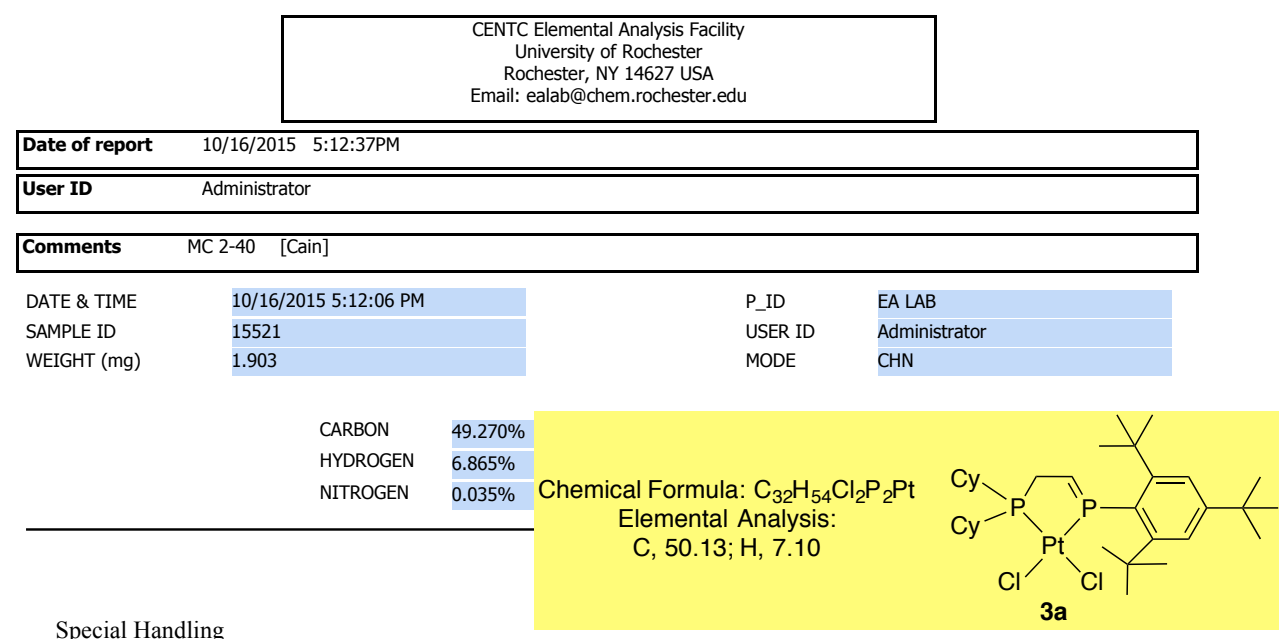

Special Handling

The sample was transferred under argon and was combusted in a tin capsule that was crimp-sealed with a die apparatus.

$\underline{\text { Acknowledgment }}$

Analytical data were obtained from the CENTC Elemental Analysis Facility at the University of Rochester, funded by NSF CHE-0650456.

$\underline{\text { Instrumentation }}$

Microanalysis samples were weighed with a PerkinElmer Model AD-6 Autobalance and their compositions were determined with a PerkinElmer 2400 Series II Analyzer. Air-sensitive samples were handled in a VAC Atmospheres glovebox 


\section{Synthesis of Pd and Pt complexes of $E-1 \mathrm{~b}$ :}

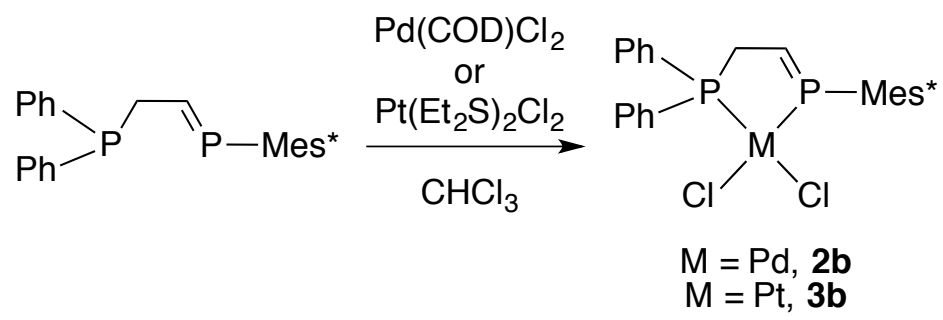

\section{Synthesis of $2 b$}

A solution of $\boldsymbol{E}-\mathbf{1 b}$ (50 $\mathrm{mg}, 0.102 \mathrm{mmol}$ ) in $1.5 \mathrm{~mL}$ of $\mathrm{CH}_{2} \mathrm{Cl}_{2}$ was added to $\mathrm{Pd}(\mathrm{COD}) \mathrm{Cl}_{2}$ (29 $\mathrm{mg}, 0.102 \mathrm{mmol}$ ), resulting in a homogeneous orange reaction mixture. After $30 \mathrm{~min}$ of stirring, ${ }^{31} \mathrm{P}\left\{{ }^{1} \mathrm{H}\right\}$ NMR revealed the metallation was complete $(\delta 238$ and 74$)$. The reaction mixture was then layered with $10 \mathrm{~mL}$ of pentane and placed in the freezer at $-35{ }^{\circ} \mathrm{C}$ affording a pale yellow solid (56 $\mathrm{mg}, 0.084 \mathrm{mmol}, 82 \%$ ).

Anal. Calcd. for $\mathrm{C}_{32} \mathrm{H}_{42} \mathrm{P}_{2} \mathrm{PdCl}_{2}$ : C, 57.71; H, 6.36. Found: C, 57.65; H, 6.51. HRMS m/z calcd. for $\mathrm{C}_{32} \mathrm{H}_{42} \mathrm{P} 2 \mathrm{PdCl}(\mathrm{M}-\mathrm{Cl})^{+}$: 629.1485. Found: 629.1492. ${ }^{31} \mathrm{P}\left\{{ }^{1} \mathrm{H}\right\} \mathrm{NMR}\left(\mathrm{CDCl}_{3}\right): \delta 236.4(\mathrm{~d}, J$ $=17, \mathrm{P}=\mathrm{C}), 74.2\left(\mathrm{~d}, J=17, \mathrm{PPh}_{2}\right) .{ }^{1} \mathrm{H}$ NMR $\left(\mathrm{CDCl}_{3}\right): \delta 7.85(\mathrm{~m}, 4 \mathrm{H}, \mathrm{Ph}), 7.54(\mathrm{~m}, 2 \mathrm{H}, \mathrm{Ph}), 7.51$ (d, $J=4,2 \mathrm{H}, \mathrm{Mes}^{*}$ ), $7.47(\mathrm{~m}, 4 \mathrm{H}, \mathrm{Ph}), 7.09$ (ddt, $J=4,14.5,56,1 \mathrm{H}, \mathrm{P}=\mathrm{CH}$ ), 3.20 (ddd, $J=4$, 10, 52, $2 \mathrm{H}, \mathrm{CH}_{2}$ backbone), $1.59(18 \mathrm{H}, t-\mathrm{Bu}), 1.31(9 \mathrm{H}, t-\mathrm{Bu}) .{ }^{13} \mathrm{C}\left\{{ }^{1} \mathrm{H}\right\} \mathrm{NMR}\left(\mathrm{CDCl}_{3}\right): \delta 156.9$ (dd, $J=64,20, \mathrm{P}=\mathrm{C}$ ), 155.9 (d, $J=3, \mathrm{Ar}$ ), $154.4(\mathrm{Ar}), 133.3$ (d, $J=12, \mathrm{Ph}$ ), $132.1(\mathrm{Ph}), 129.0$ (d, $J=12, \mathrm{Ph}$ ), 128.5 (d, $J=56, \mathrm{Ph}), 123.8$ (d, $J=11, \mathrm{Ar}), 122.1$ (d, $J=16, \mathrm{Ar}$ ), $39.1\left(C \mathrm{Ce}_{3}\right)$, $36.4\left(\mathrm{dd}, J=35,23, \mathrm{CH}_{2}\right.$ backbone), $35.3\left(\mathrm{CMe}_{3}\right), 34.9(t-\mathrm{Bu}), 31.0(t-\mathrm{Bu})$. 
NMR Spectroscopy for 2b

${ }^{31} \mathrm{P}\left\{{ }^{1} \mathrm{H}\right\}$ NMR $\left(\mathrm{CDCl}_{3}\right)$

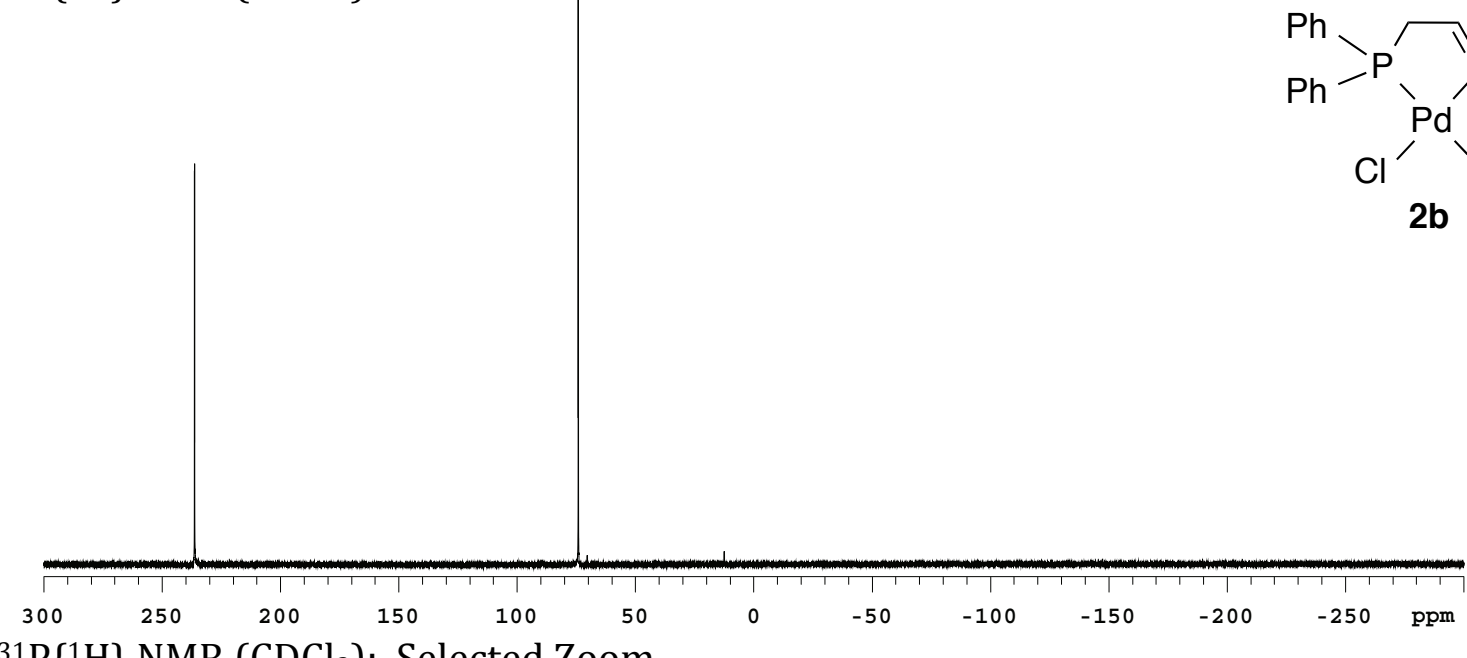

${ }^{31} \mathrm{P}\left\{{ }^{1} \mathrm{H}\right\}$ NMR $\left(\mathrm{CDCl}_{3}\right)$ : Selected Zoom

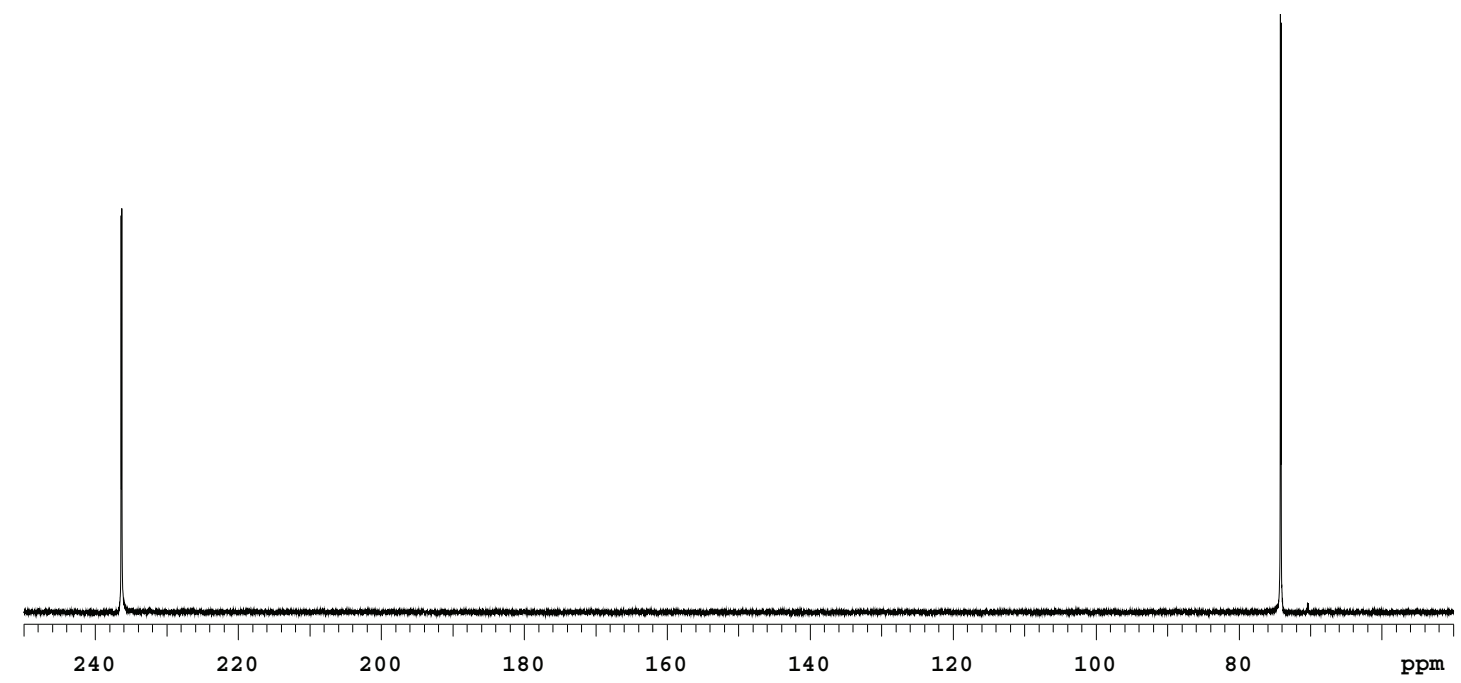


${ }^{1} \mathrm{H} \mathrm{NMR}\left(\mathrm{CDCl}_{3}\right)$

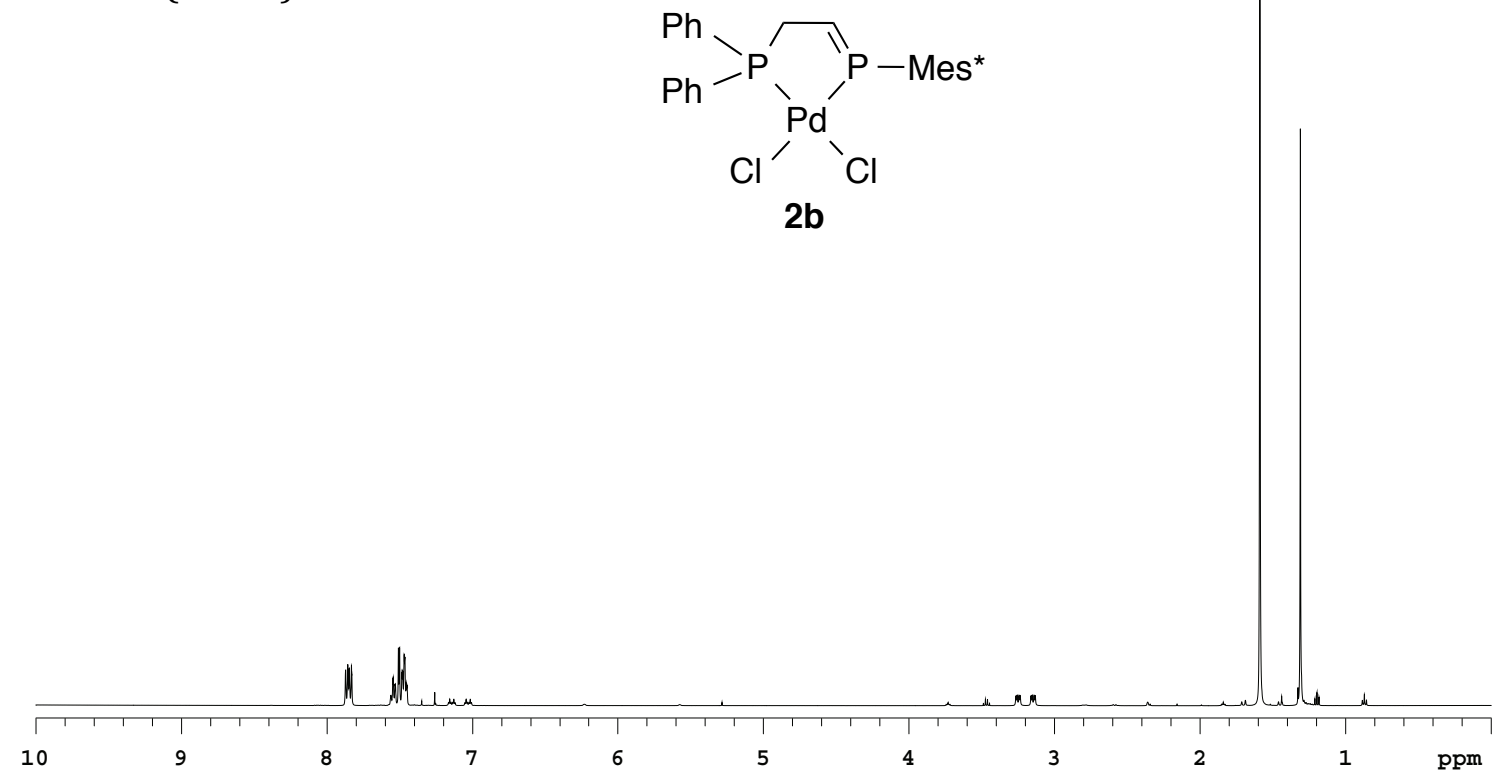

${ }^{1} \mathrm{H} \mathrm{NMR}\left(\mathrm{CDCl}_{3}\right)$ : Selected Zoom

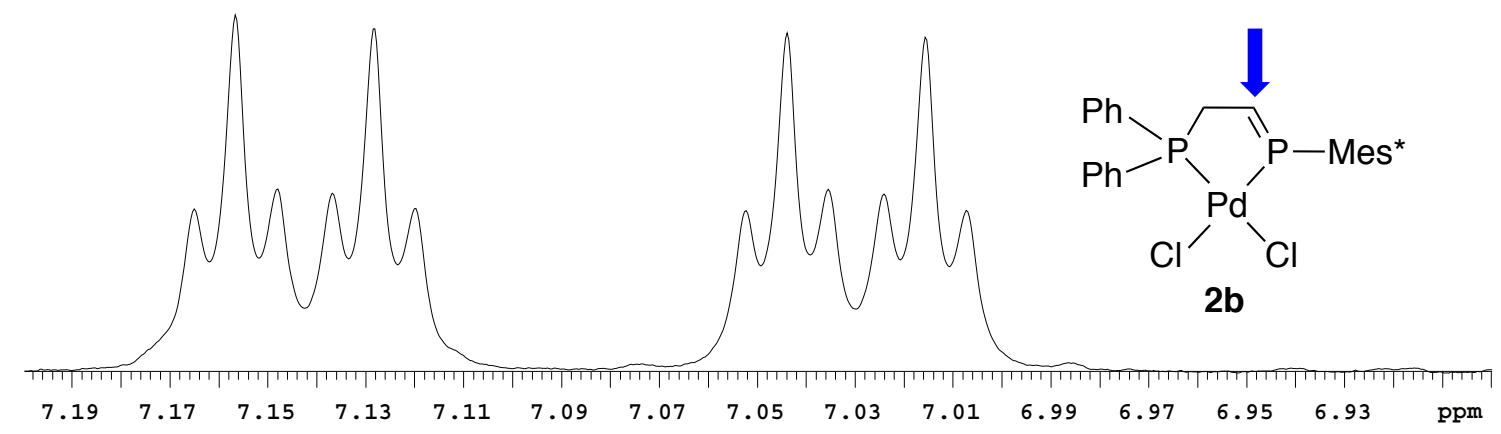

${ }^{1} \mathrm{H} \mathrm{NMR}\left(\mathrm{CDCl}_{3}\right)$ : Selected Zoom

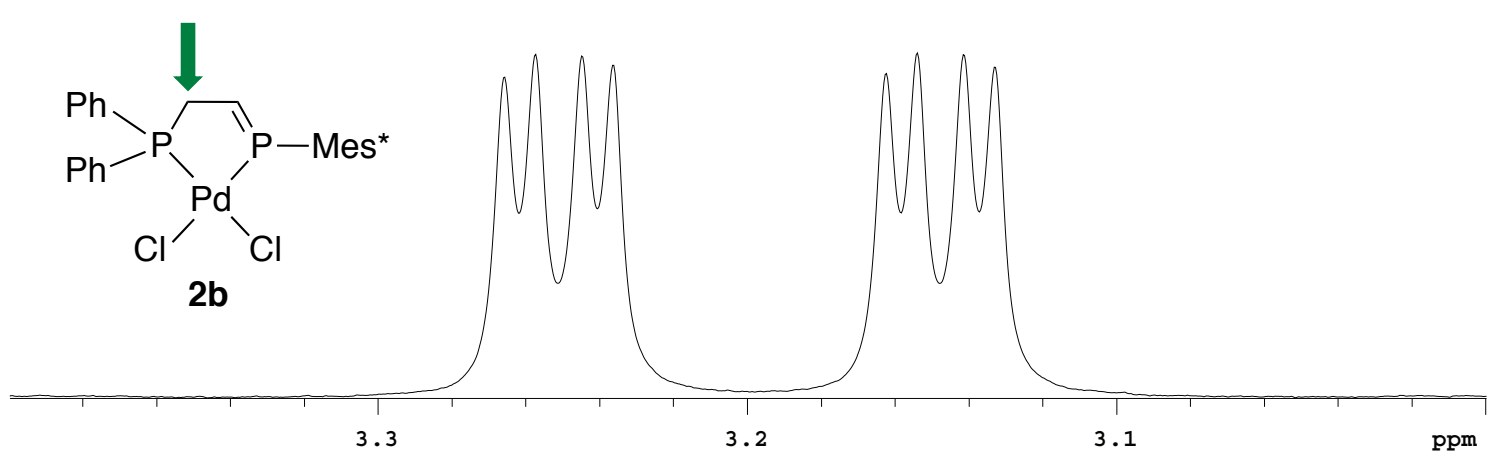


${ }^{13} \mathrm{C}\left\{{ }^{1} \mathrm{H}\right\} \mathrm{NMR}\left(\mathrm{CDCl}_{3}\right)$

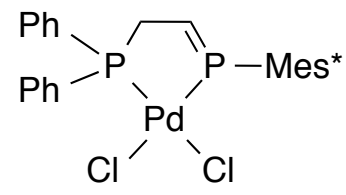

2b

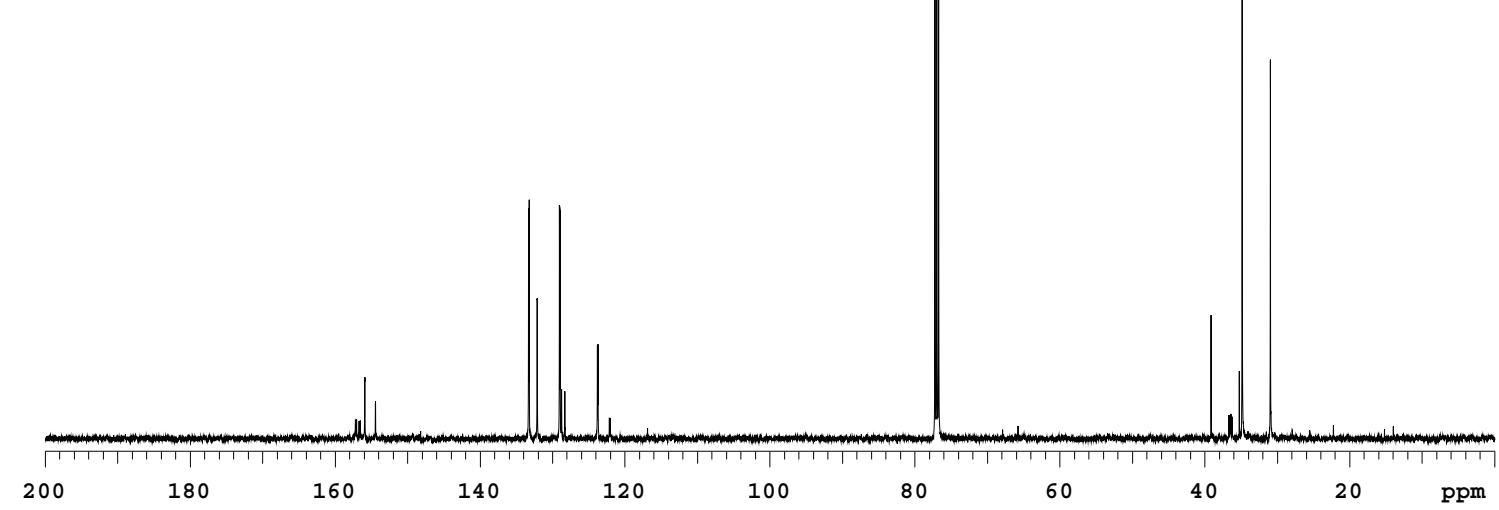

${ }^{13} \mathrm{C}\left\{{ }^{1} \mathrm{H}\right\}$ NMR $\left(\mathrm{CDCl}_{3}\right)$ : Selected Zoom

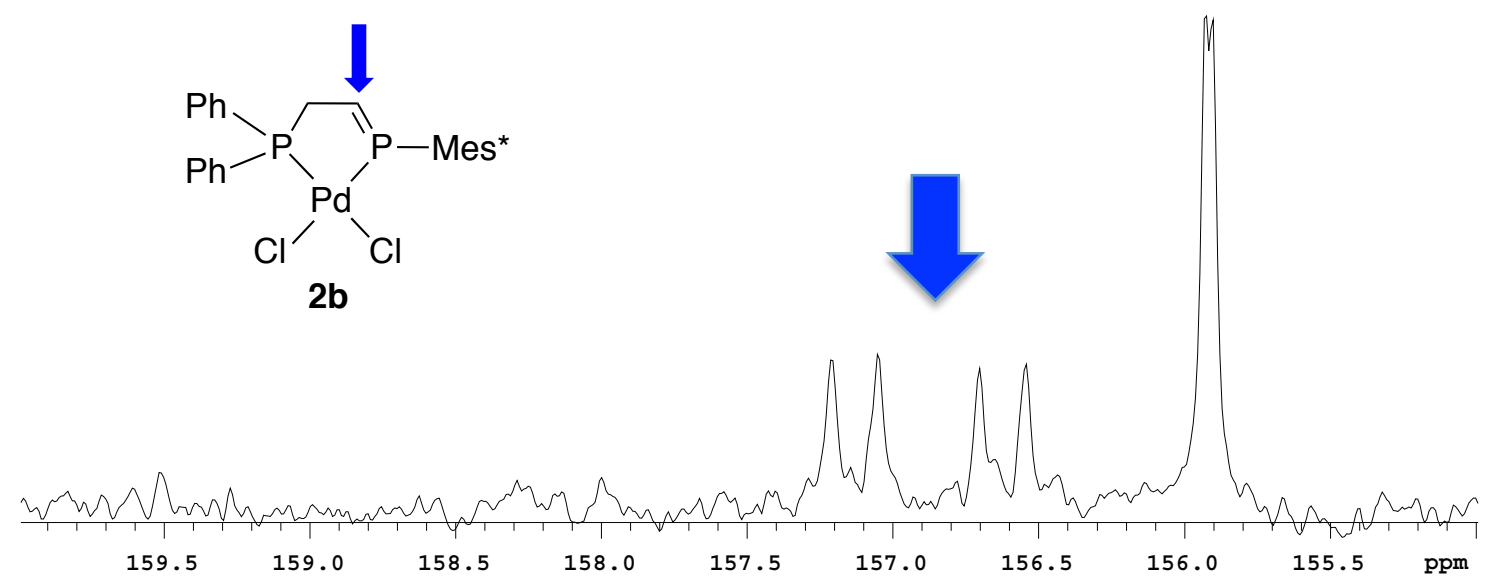


${ }^{13} \mathrm{C}\left\{{ }^{1} \mathrm{H}\right\}$ NMR $\left(\mathrm{CDCl}_{3}\right)$ : Selected Zoom

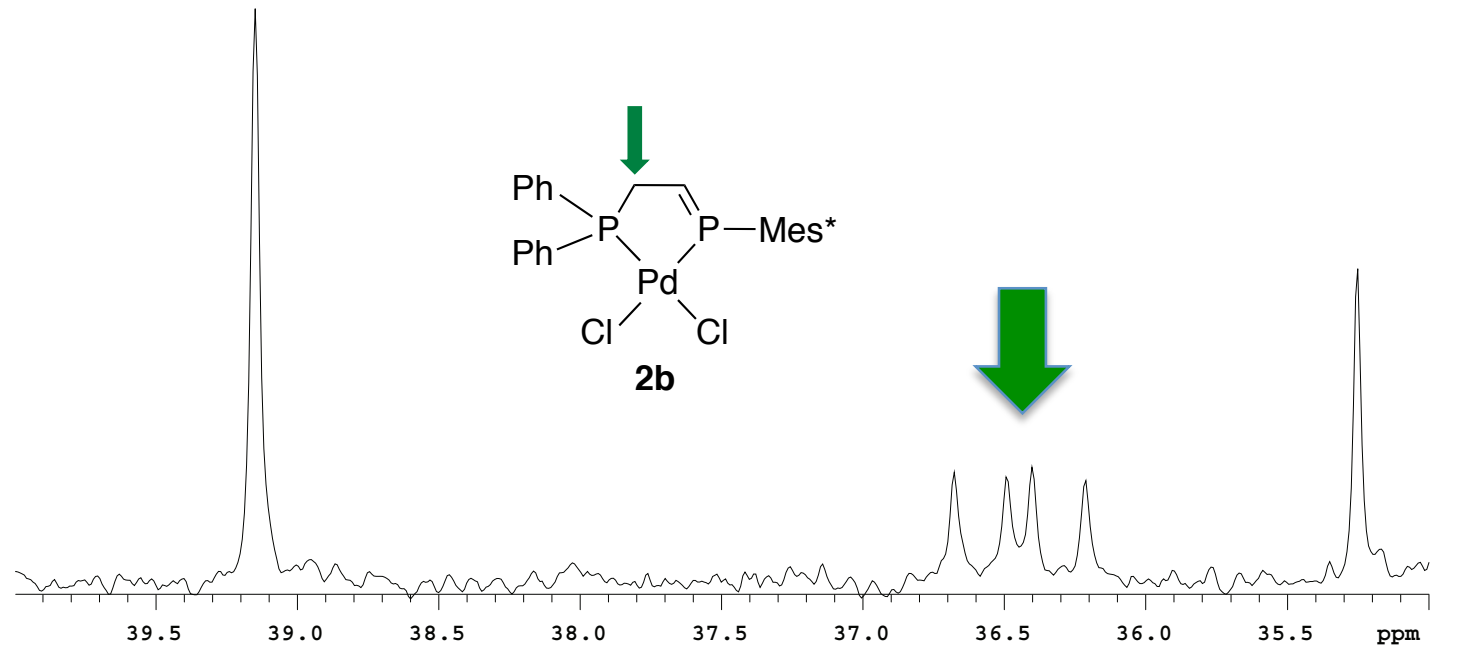




\section{Elemental Analysis for $2 b$}

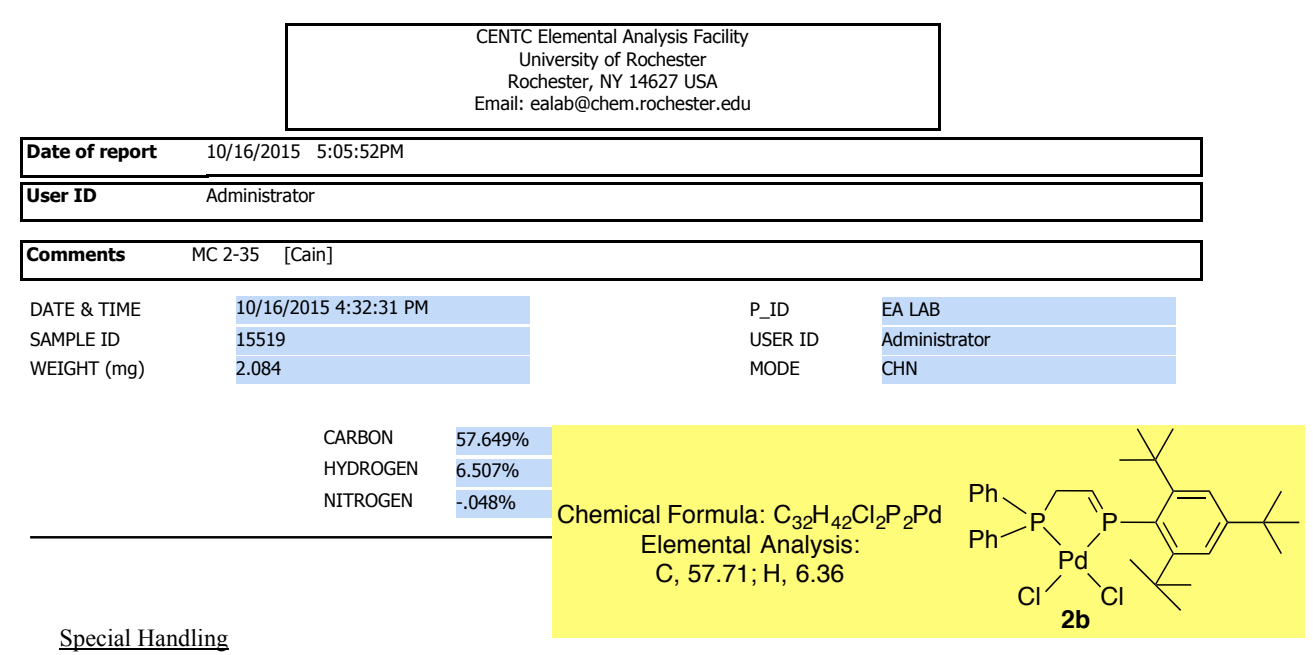

The sample was transferred under argon and was combusted in a tin capsule that was crimp-sealed with a die apparatus.

Acknowledgment

Analytical data were obtained from the CENTC Elemental Analysis Facility at the University of Rochester, funded by NSF CHE-0650456.

Instrumentation

Microanalysis samples were weighed with a PerkinElmer Model AD-6 Autobalance and their compositions were determined with a PerkinElmer 2400 Series II Analyzer. Air-sensitive samples were handled in a VAC

Atmospheres glovebox. 


\section{Synthesis of $\mathbf{3 b}$}

A solution of $\boldsymbol{E}-\mathbf{1 b}(50 \mathrm{mg}, 0.102 \mathrm{mmol})$ in $1.5 \mathrm{~mL}$ of $\mathrm{CH}_{2} \mathrm{Cl}_{2}$ was added to $\mathrm{Pt}\left(\mathrm{Et}_{2} \mathrm{~S}\right)_{2} \mathrm{Cl}_{2}(46$ $\mathrm{mg}, 0.103 \mathrm{mmol}$ ), resulting in a homogeneous orange reaction mixture. After $30 \mathrm{~min}$ of stirring, the solution was layered with $10 \mathrm{~mL}$ of pentane and placed in the freezer at $-35{ }^{\circ} \mathrm{C}$ affording a beige solid (55 $\mathrm{mg}, 0.073 \mathrm{mmol}, 71 \%$ ). X-ray quality crystals were obtained by exposing a concentrated solution of $\mathbf{3 b}$ in THF (15 mg, $1 \mathrm{~mL})$ to diethyl ether vapors at -35 ${ }^{\circ} \mathrm{C}$.

Anal. Calcd. for $\mathrm{C}_{32} \mathrm{H}_{42} \mathrm{P}_{2} \mathrm{PtCl}_{2}$ : C, 50.93; H, 5.61. Found: C, 51.12; H, 5.59. HRMS $m / z$ calcd for $\mathrm{C}_{32} \mathrm{H}_{42} \mathrm{P}_{2} \mathrm{PtCl}_{2}(\mathrm{M}-\mathrm{Cl})^{+}$: 718.2098. Found: 718.2100. ${ }^{31} \mathrm{P}\left\{{ }^{1} \mathrm{H}\right\} \mathrm{NMR}\left(\mathrm{CDCl}_{3}\right): \delta 217.1(\mathrm{~d}$, $\left.J_{\mathrm{PP}}=7, J_{\mathrm{Pt}-\mathrm{P}}=4425, \mathrm{P}=\mathrm{C}\right), 47.1\left(\mathrm{~d}, J_{\mathrm{PP}}=7, J_{\mathrm{Pt}-\mathrm{P}}=3390, \mathrm{PPh}_{2}\right) .{ }^{1} \mathrm{H}$ NMR $\left(\mathrm{CDCl}_{3}\right): \delta 7.84(\mathrm{~m}, 4 \mathrm{H}$, $\mathrm{Ph}$ ), 7.54 (overlapping multiplet, 4H, Ph and Mes*), 7.49 (m, 4H, Ph), 7.28 (ddt, $J=4,9,47$, $1 \mathrm{H}, \mathrm{P}=\mathrm{CH}$ ), $2.76\left(\mathrm{ddd}, J=4,10.5,47,2 \mathrm{H}\right.$, backbone $\left.\mathrm{CH}_{2}\right), 1.63(18 \mathrm{H}, t-\mathrm{Bu}), 1.32(9 \mathrm{H}, t-\mathrm{Bu})$. ${ }^{13} \mathrm{C}\left\{{ }^{1} \mathrm{H}\right\}$ NMR $\left(\mathrm{CDCl}_{3}\right): \delta 156.3(\mathrm{Ar}), 154.4(\mathrm{Ar}), 149.3(\mathrm{dd}, J=84,12, \mathrm{P}=\mathrm{C}), 133.2(\mathrm{~d}, J=10$, $\mathrm{Ph}), 132.0(\mathrm{Ph}), 128.9$ (d, $J=12, \mathrm{Ph}), 128.2(\mathrm{~d}, J=64, \mathrm{Ph}), 123.9(\mathrm{~d}, J=11, \mathrm{Ar}), 120.0(\mathrm{~d}, J=$ 36, $\mathrm{Ar}), 39.3\left(\mathrm{CMe}_{3}\right), 35.8\left(\mathrm{dd}, J=42,14\right.$, backbone $\left.\mathrm{CH}_{2}\right), 35.3\left(C \mathrm{Me}_{3}\right), 34.8(t-\mathrm{Bu}), 31.0(t$ $\mathrm{Bu})$. 
NMR Spectroscopy for 3b

${ }^{31} \mathrm{P}\left\{{ }^{1} \mathrm{H}\right\} \mathrm{NMR}\left(\mathrm{CDCl}_{3}\right)$
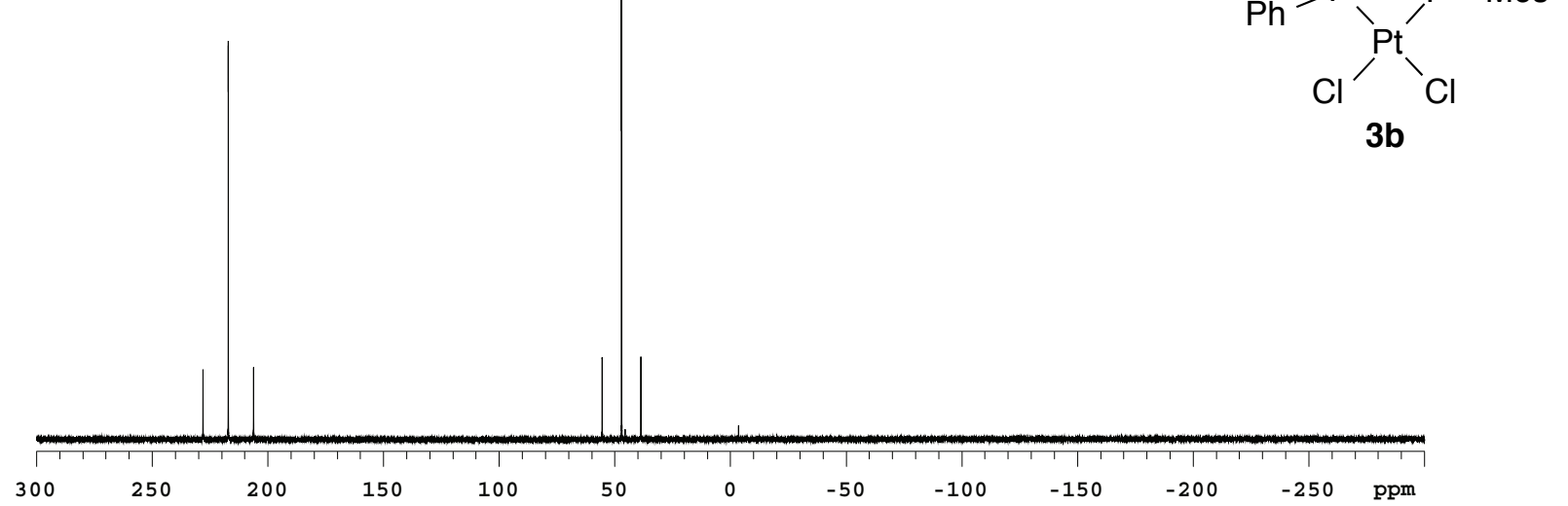

${ }^{31} \mathrm{P}\left\{{ }^{1} \mathrm{H}\right\}$ NMR $\left(\mathrm{CDCl}_{3}\right)$ : Selected Zoom

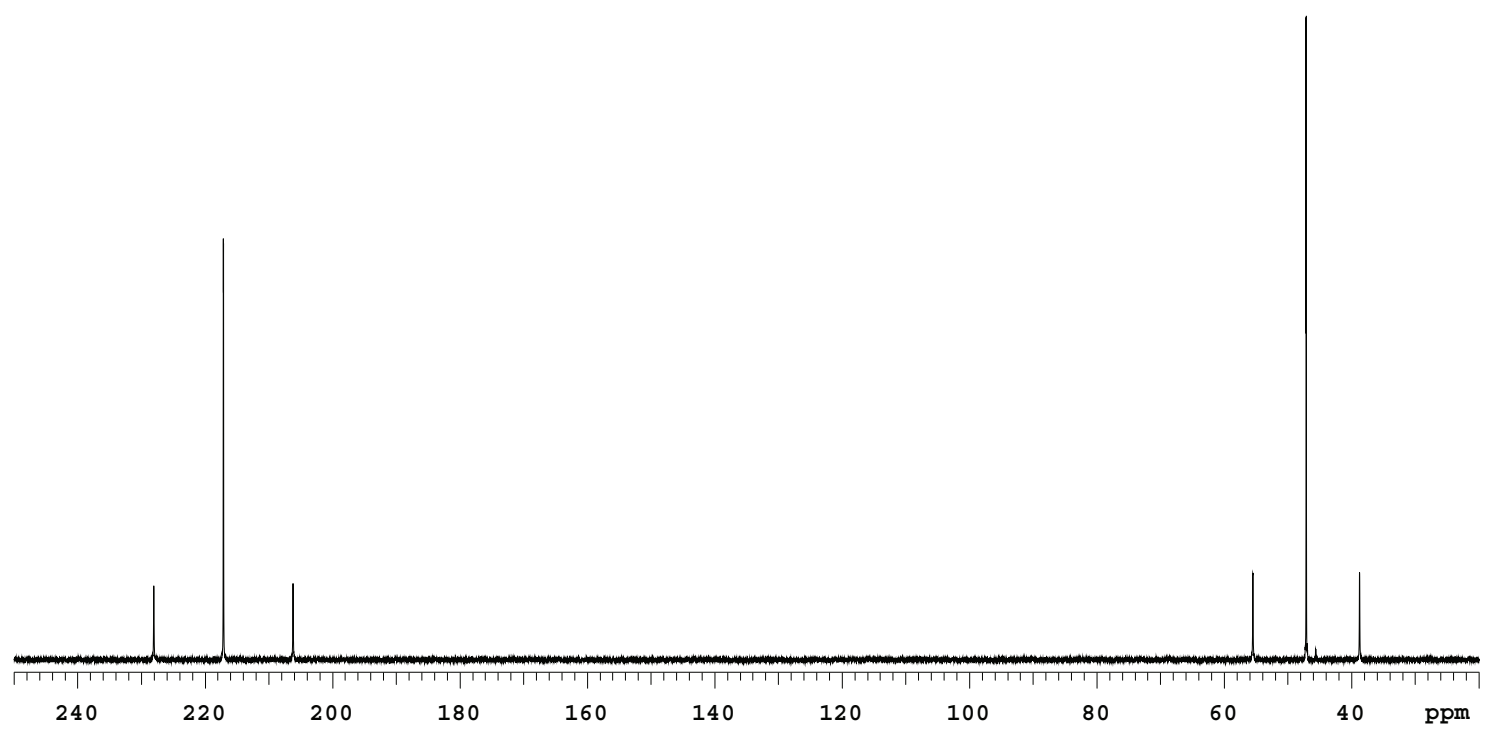


${ }^{1} \mathrm{H} \mathrm{NMR}\left(\mathrm{CDCl}_{3}\right)$

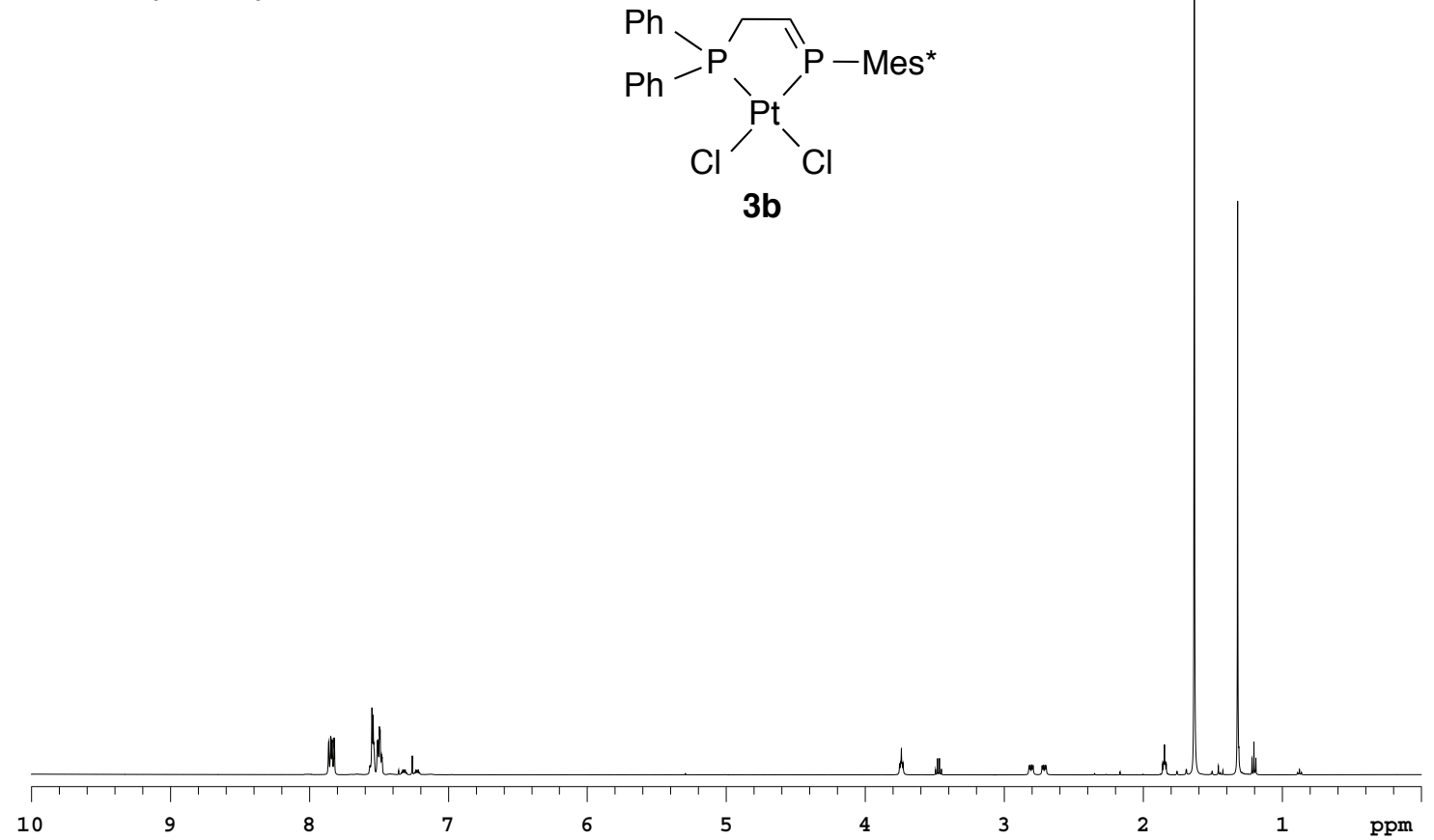

${ }^{1} \mathrm{H} \mathrm{NMR}\left(\mathrm{CDCl}_{3}\right)$ : Selected Zoom (large singlet is chloroform)

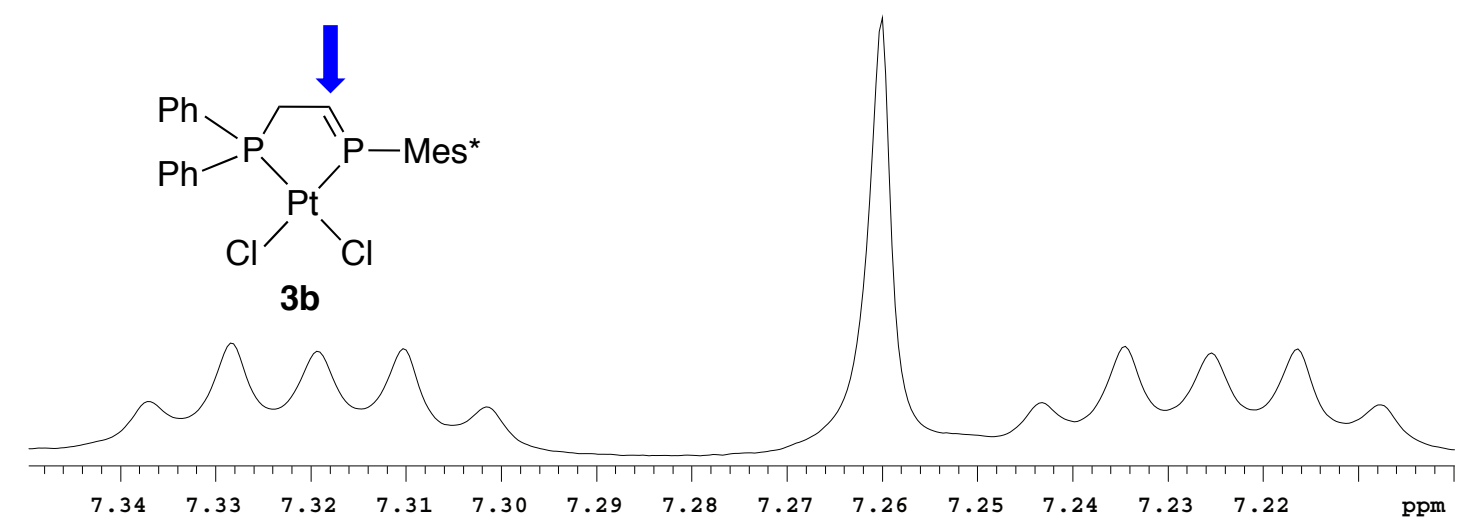

${ }^{1} \mathrm{H} \mathrm{NMR}\left(\mathrm{CDCl}_{3}\right)$ : Selected Zoom

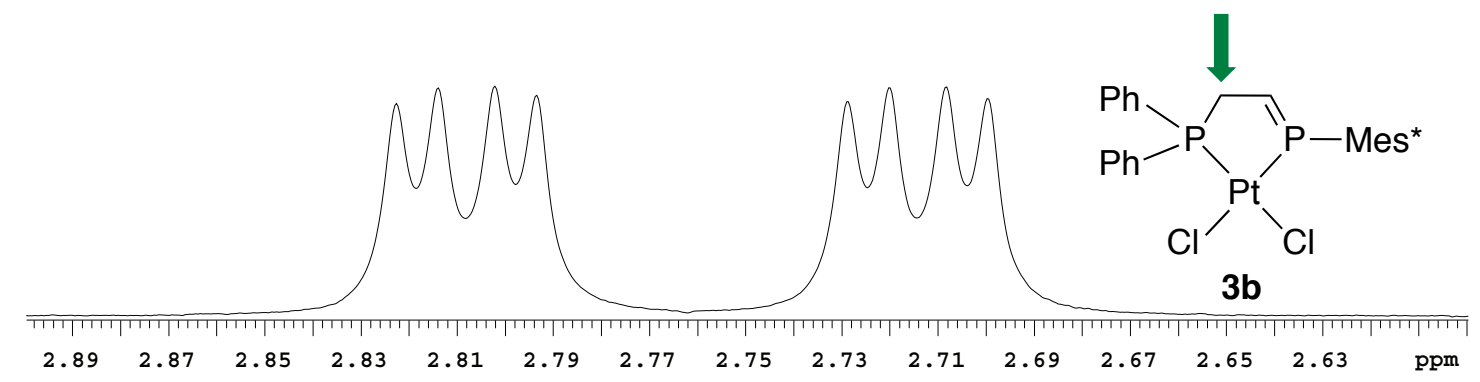


${ }^{13} \mathrm{C}\left\{{ }^{1} \mathrm{H}\right\}$ NMR $\left(\mathrm{CDCl}_{3}\right)$
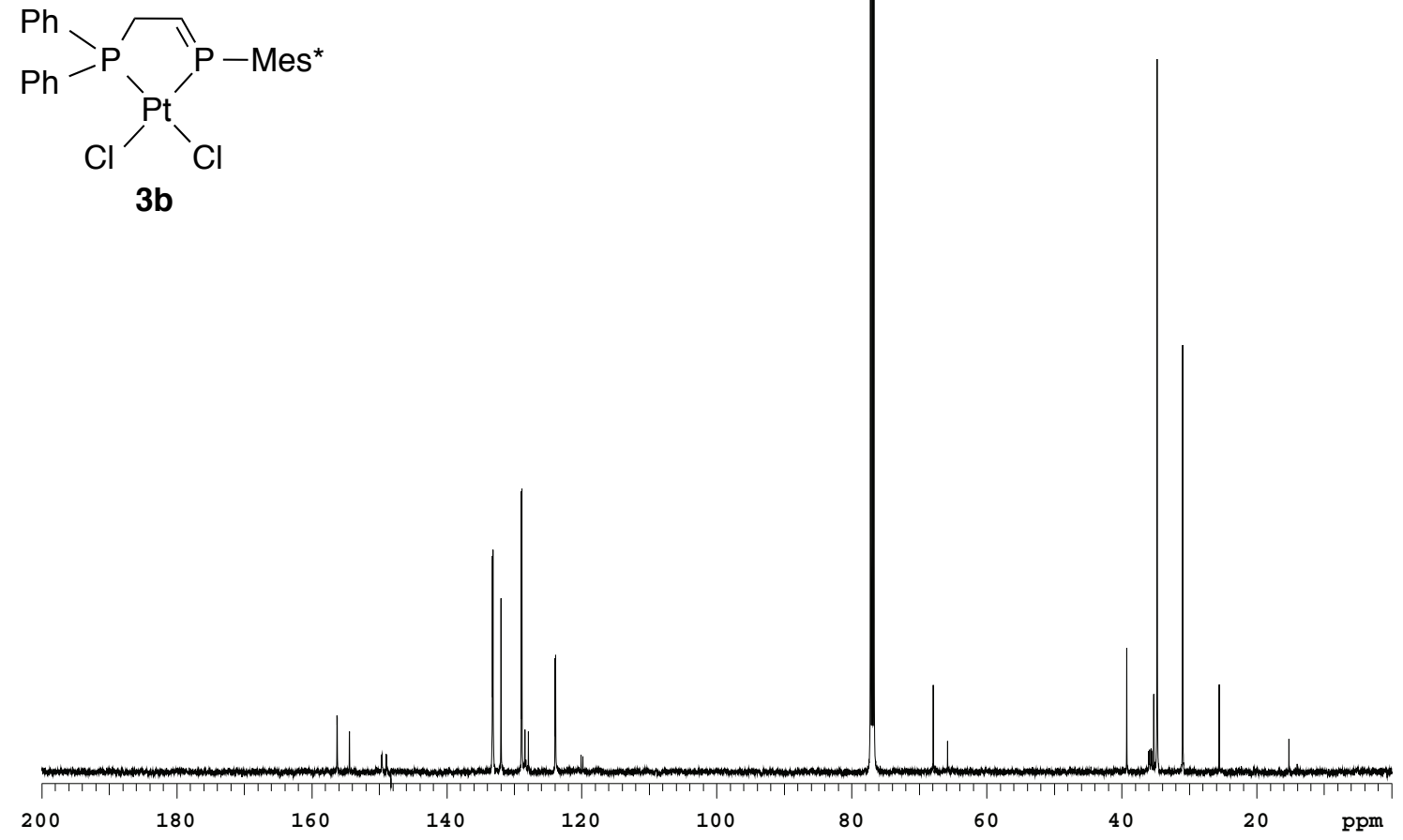

${ }^{13} \mathrm{C}\left\{{ }^{1} \mathrm{H}\right\}$ NMR $\left(\mathrm{CDCl}_{3}\right)$ : Selected Zoom

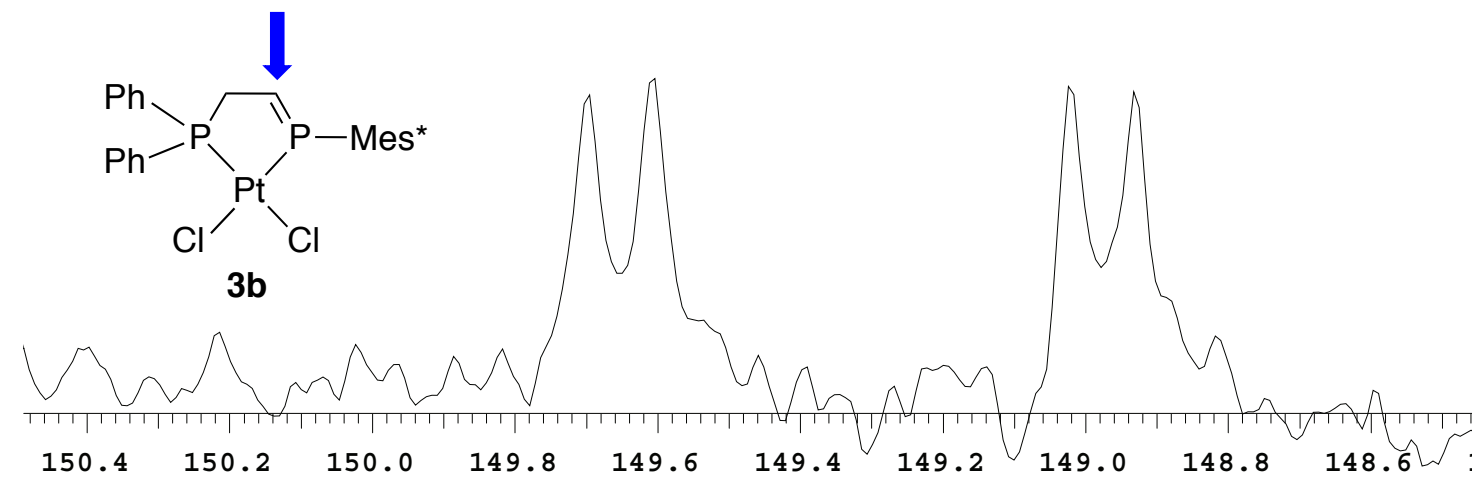


${ }^{13} \mathrm{C}\left\{{ }^{1} \mathrm{H}\right\}$ NMR $\left(\mathrm{CDCl}_{3}\right)$ : Selected Zoom

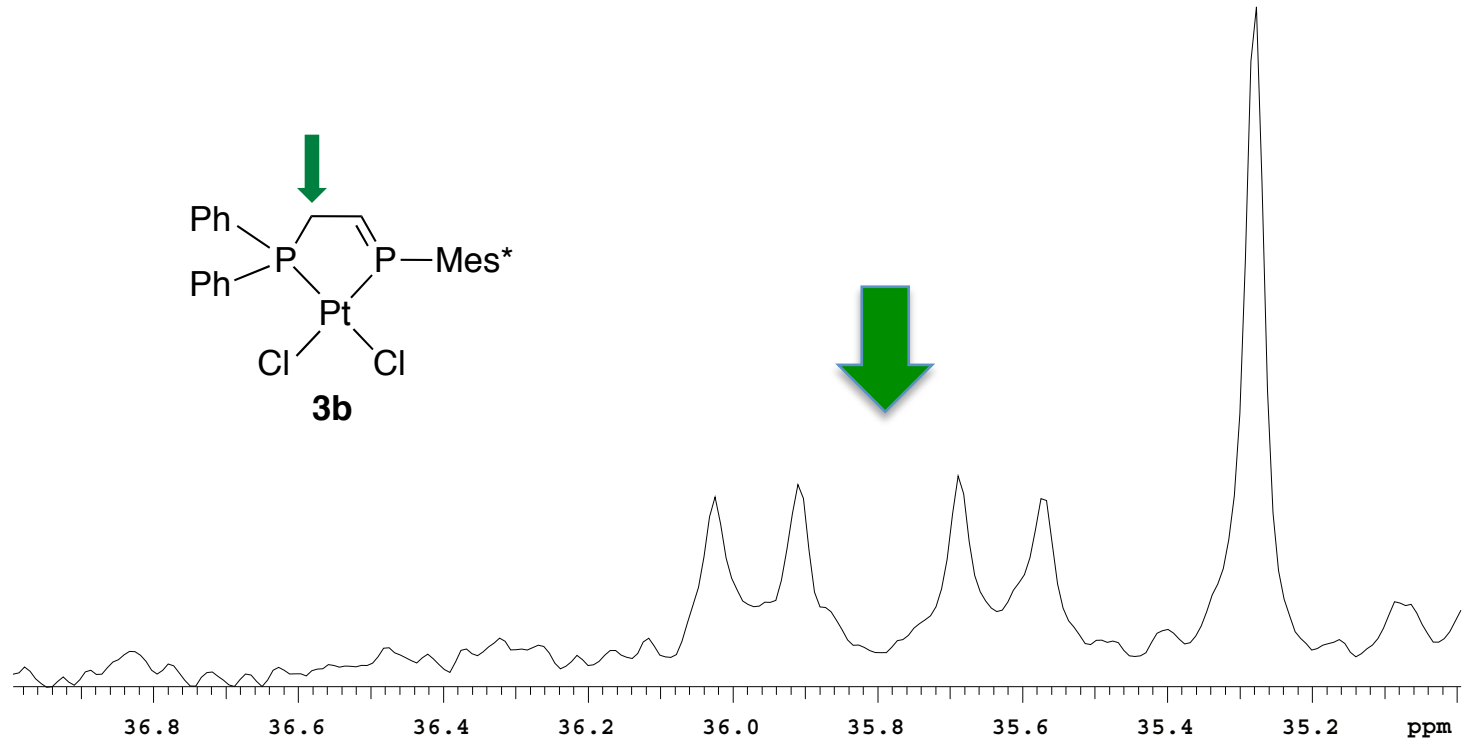




\section{Elemental Analysis for $3 b$}

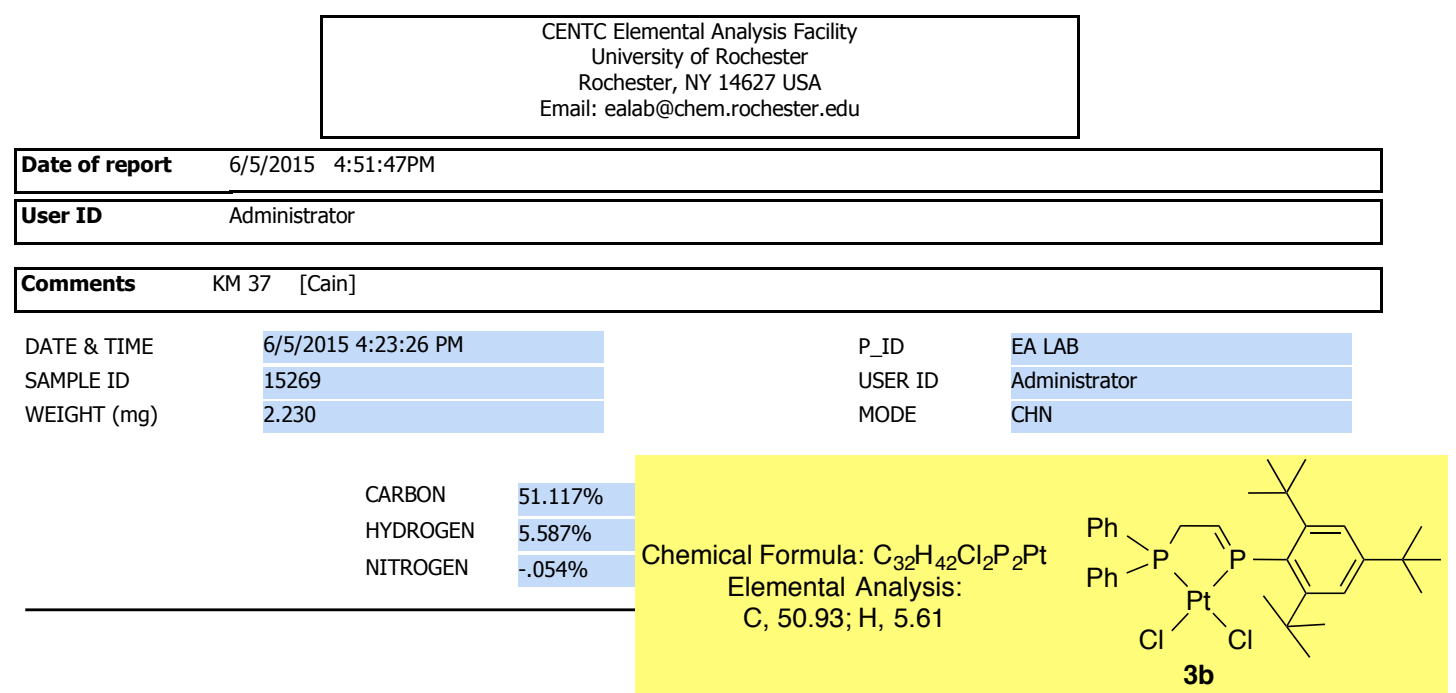

$\underline{\text { Special Handling }}$

The sample was transferred under argon and was combusted in a tin capsule that was crimp-sealed with a die apparatus.

\section{$\underline{\text { Acknowledgment }}$}

Analytical data were obtained from the CENTC Elemental Analysis Facility at the University of Rochester, funded by NSF CHE-0650456.

\section{$\underline{\text { Instrumentation }}$}

Microanalysis samples were weighed with a PerkinElmer Model AD-6 Autobalance and their compositions were determined with a PerkinElmer 2400 Series II Analyzer. Air-sensitive samples were handled in a VAC

Atmospheres glovebox. 


\section{DFT Computational Details}

Full-molecule calculations were carried out using the hybrid M06 functional ${ }^{1}$ and the LACV3P ${ }^{* *}$ basis, ${ }^{2}$ as implemented in the Jaguar ${ }^{3}$ suite of programs. Natural Bond Orbital calculations ${ }^{4}$ were performed on the optimized structure using NBO $6.0,5$ as implemented in Jaguar. Computed structures were confirmed as minima by calculating the vibrational frequencies using second derivative analytic methods, and confirming the absence of imaginary frequencies for minima. Thermodynamic quantities were calculated assuming an ideal gas, and are zero point energy corrected. Graphical representations of structures were made using the CYLView program. ${ }^{6}$

\section{Material Relevant to all DFT output}

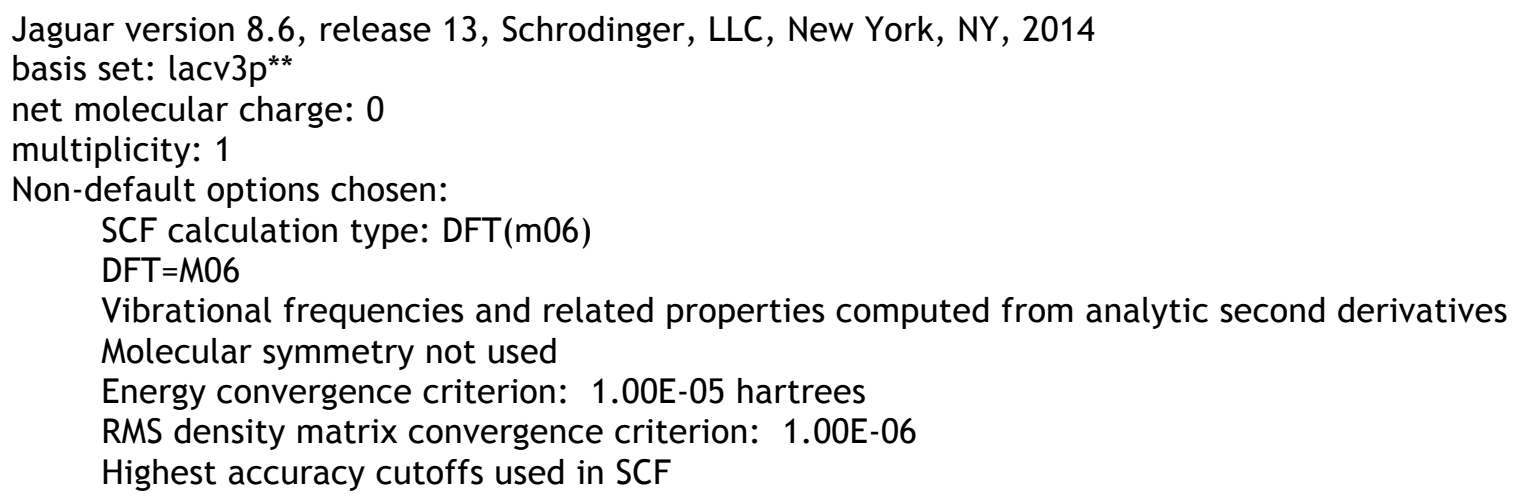

\section{References.}

(1) (a) Zhao, Y.; Truhlar, D. G. Theor. Chem. Acc. 2008, 120, 215-241; (b) Zhao, Y.; Truhlar, D. G. Acc. Chem. Res. 2008, 41, 157-167.

(2) (a) Dunning, T. H.; Hay, P. J., In Modern Theoretical Chemistry, Vol. 4: Applications of Electronic Structure Theory. Schaefer, H. F., III (Editor); Plenum, NY: 1977 ; (b) Wadt, W. R.; Hay, P. J. J. Chem. Phys. 1985, 82, 284-298; (c) Hay, P. J.; Wadt, W. R. J. Chem. Phys. 1985, 82, 299-310; (d) Hay, P. J.; Wadt, W. R. J. Chem. Phys. 1985, 82, 270-283.

(3) (a) Bochevarov, A. D.; Harder, E.; Hughes, T. F.; Greenwood, J. R.; Braden, D. A.; Philipp, D. M.; Rinaldo, D.; Halls, M. D.; Zhang, J.; Friesner, R. A. Int. J. Quantum Chem. 2013, 113, 2110-2142; (b) Jaguar, versions 7.0-8.9, Schrödinger, LLC, New York, NY: 2007-2015.

(4) (a) Weinhold, F.; Landis, C. R., Discovering Chemistry with Natural Bond Orbitals, John Wiley \& Sons: NJ, 2012; (b) Glendening, E. D.; Landis, C. R.; Weinhold, F. Wiley Interdisciplinary Reviews: Computational Molecular Science 2012, 2, 1-42; (c) Weinhold, F.; Landis, C. R., Valency and Bonding: A Natural Bond Orbital Donor-Acceptor Perspective, Cambridge University Press: Cambridge, 2005; (d) Reed, A. E.; Curtiss, L. A.; Weinhold, F. Chem. Rev. 1988, 88, 899-926.

(5) (a) Glendening, E. D.; Landis, C. R.; Weinhold, F. J. Comp. Chem. 2013, 34, 1429-1437; (b) Glendening, E. D.; Badenhoop, J. K.; Reed, A. K.; Carpenter, J. E.; Bohmann, J. A.; Morales, C. M.; Landis, C. R.; Weinhold, F. NBO 6.0, Theoretical Chemistry Institute, University of Wisconsin; Madison: 2013.

(6) Legault, C. Y. CYLview, 1.0b, Université de Sherbrooke: Université de Sherbrooke, 2009. 
XYZ Coordinate files for DFT optimized structures

E-1a-gauche

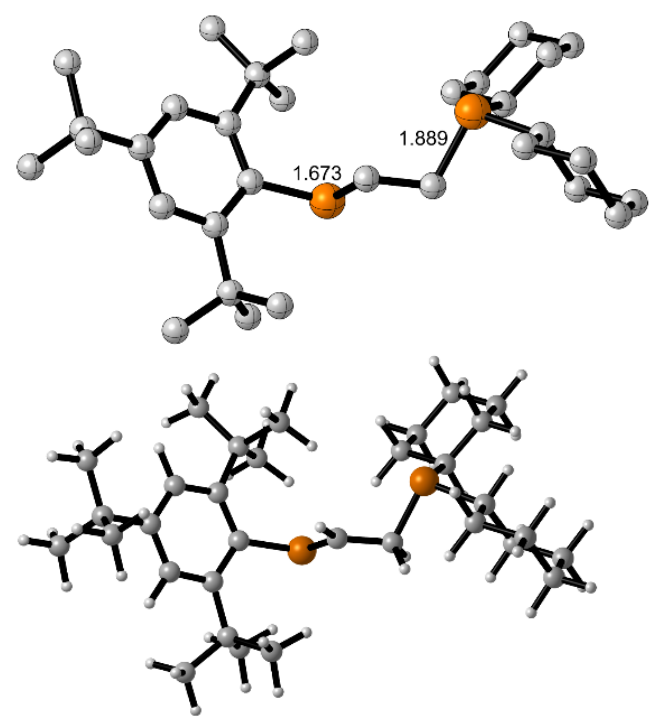

\begin{tabular}{|c|c|c|c|}
\hline $\mathrm{H}$ & 10.74500 & -2.92690 & 14.67920 \\
\hline C & 8.80800 & -2.29670 & 15.41470 \\
\hline $\mathrm{H}$ & 8.94210 & -2.80150 & 16.38370 \\
\hline $\mathrm{H}$ & 8.24650 & -2.98950 & 14.77680 \\
\hline$C$ & 8.01140 & -1.01570 & 15.6275 \\
\hline $\mathrm{H}$ & 7.04140 & -1.23560 & 16.0909 \\
\hline $\mathrm{H}$ & 7.79070 & -0.57190 & 14.6432 \\
\hline C & 4.69390 & 5.07650 & 14.31700 \\
\hline C & 4.22800 & 5.01590 & 12.9733 \\
\hline C & 2.85610 & 4.94050 & 12.7615 \\
\hline $\mathrm{H}$ & 2.48600 & 4.85750 & 11.7477 \\
\hline C & 1.92180 & 4.96310 & 13.7882 \\
\hline C & 2.40230 & 5.14420 & 15.0708 \\
\hline $\mathrm{H}$ & 1.68740 & 5.21780 & 15.8776 \\
\hline C & 3.76430 & 5.22930 & 15.37350 \\
\hline C & 6.19210 & 6.14270 & 11.8025 \\
\hline $\mathrm{H}$ & 5.72030 & 7.11570 & 11.98260 \\
\hline $\mathrm{H}$ & 6.73910 & 6.20160 & 10.8541 \\
\hline $\mathrm{H}$ & 6.93680 & 5.97830 & 12.58450 \\
\hline C & 5.79510 & 3.67420 & 11.4785 \\
\hline $\mathrm{H}$ & 6.52030 & 3.41440 & 12.25160 \\
\hline $\mathrm{H}$ & 6.31840 & 3.67970 & 10.51410 \\
\hline $\mathrm{H}$ & 5.03630 & 2.88300 & 11.44630 \\
\hline C & 4.33130 & 5.34910 & 10.44610 \\
\hline $\mathrm{H}$ & 3.76120 & 6.28080 & 10.53680 \\
\hline $\mathrm{H}$ & 3.64380 & 4.54270 & 10.17090 \\
\hline $\mathrm{H}$ & 5.02940 & 5.46600 & 9.61070 \\
\hline C & 5.13330 & 5.03740 & 11.71710 \\
\hline C & -0.43860 & 4.87690 & 14.71420 \\
\hline $\mathrm{H}$ & -0.19790 & 4.06440 & 15.40930 \\
\hline $\mathrm{H}$ & -1.49040 & 4.76550 & 14.4277 \\
\hline $\mathrm{H}$ & -0.34250 & 5.82920 & 15.2482 \\
\hline C & 0.00830 & 5.98340 & 12.5492 \\
\hline $\mathrm{H}$ & -1.06110 & 5.90670 & 12.31740 \\
\hline $\mathrm{H}$ & 0.55550 & 5.97970 & 11.6010 \\
\hline $\mathrm{H}$ & 0.18410 & 6.95080 & 13.0335 \\
\hline C & 0.18490 & 3.49760 & 12.7604 \\
\hline $\mathrm{H}$ & 0.48340 & 2.65910 & 13.40000 \\
\hline $\mathrm{H}$ & 0.74190 & 3.41690 & 11.82160 \\
\hline $\mathrm{H}$ & -0.88090 & 3.38620 & 12.5265 \\
\hline C & 0.43640 & 4.83350 & 13.46540 \\
\hline C & 2.95320 & 6.11260 & 17.62300 \\
\hline $\mathrm{H}$ & 3.29490 & 6.38330 & 18.62760 \\
\hline $\mathrm{H}$ & 2.10230 & 5.43790 & 17.7562 \\
\hline $\mathrm{H}$ & 2.60070 & 7.02610 & 17.13060 \\
\hline C & 4.41030 & 4.13110 & 17.55740 \\
\hline $\mathrm{H}$ & 4.58220 & 4.29580 & 18.62940 \\
\hline $\mathrm{H}$ & 5.28740 & 3.62450 & 17.1497 \\
\hline $\mathrm{H}$ & 3.55460 & 3.45330 & 17.4542 \\
\hline C & 5.29010 & 6.43830 & 17.0468 \\
\hline $\mathrm{H}$ & 5.42890 & 6.64000 & 18.1158 \\
\hline $\mathrm{H}$ & 5.08370 & 7.39190 & 16.5463 \\
\hline $\mathrm{H}$ & 6.24770 & 6.06770 & 16.6752 \\
\hline C & 4.12060 & 5.46540 & 16.8615 \\
\hline
\end{tabular}


E-1a-cis

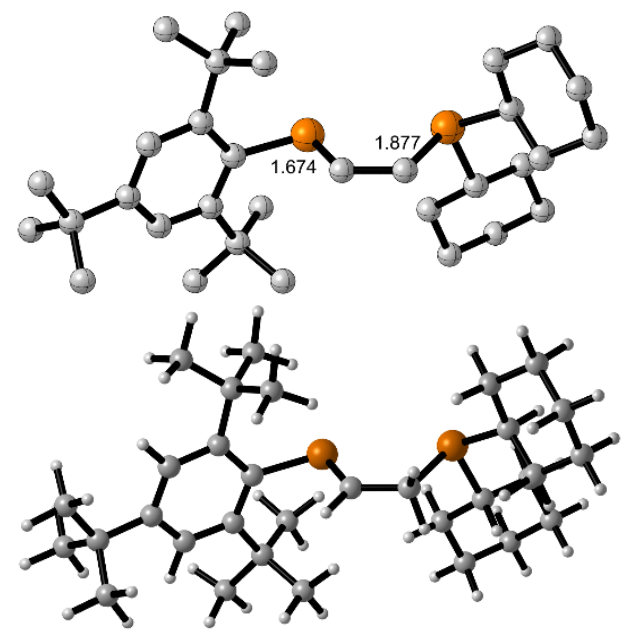

\begin{tabular}{|c|c|c|c|}
\hline C & 11.33280 & 3.88780 & 18.55550 \\
\hline r & 12.35180 & 4.12510 & 18.21330 \\
\hline $\mathrm{H}$ & 11.11680 & 4.56810 & 19.3878 \\
\hline C & 10.35630 & 4.13020 & 17.41160 \\
\hline $\mathrm{H}$ & 10.42260 & 5.16710 & 17.05900 \\
\hline $\mathrm{H}$ & 9.32920 & 3.99860 & 17.7898 \\
\hline C & 4.68550 & 5.11320 & 13.8270 \\
\hline C & 3.91500 & 4.37800 & 12.88560 \\
\hline C & 2.53240 & 4.36730 & 13.0407 \\
\hline $\mathrm{H}$ & 1.93370 & 3.78200 & 12.35420 \\
\hline C & 1.86970 & 5.07020 & 14.03620 \\
\hline C & 2.64010 & 5.86460 & 14.86600 \\
\hline $\mathrm{H}$ & 2.13500 & 6.45790 & 15.61410 \\
\hline C & 4.03310 & 5.93070 & 14.78310 \\
\hline C & 5.60610 & 4.31950 & 10.96220 \\
\hline $\mathrm{H}$ & 5.25940 & 5.30790 & 10.63810 \\
\hline $\mathrm{H}$ & 5.90000 & 3.75440 & 10.0695 \\
\hline $\mathrm{H}$ & 6.51370 & 4.46210 & 11.55200 \\
\hline C & 4.97460 & 2.19240 & 12.16160 \\
\hline $\mathrm{H}$ & 5.77090 & 2.26000 & 12.90530 \\
\hline $\mathrm{H}$ & 5.35450 & 1.61830 & 11.30590 \\
\hline $\mathrm{H}$ & 4.14690 & 1.62800 & 12.60760 \\
\hline C & 3.42140 & 3.30990 & 10.62140 \\
\hline $\mathrm{H}$ & 2.94720 & 4.23840 & 10.28330 \\
\hline $\mathrm{H}$ & 2.63980 & 2.61990 & 10.95370 \\
\hline $\mathrm{H}$ & 3.90070 & 2.84350 & 9.75430 \\
\hline C & 4.48800 & 3.56960 & 11.69550 \\
\hline C & -0.20470 & 5.82120 & 15.29020 \\
\hline $\mathrm{H}$ & 0.19790 & 5.51310 & 16.26190 \\
\hline $\mathrm{H}$ & -1.29370 & 5.70700 & 15.33310 \\
\hline $\mathrm{H}$ & 0.00900 & 6.88690 & 15.14930 \\
\hline C & -0.29370 & 5.45570 & 12.84740 \\
\hline $\mathrm{H}$ & -1.38670 & 5.40030 & 12.92110 \\
\hline $\mathrm{H}$ & 0.01270 & 4.85100 & 11.9878 \\
\hline $\mathrm{H}$ & -0.01770 & 6.49590 & 12.64010 \\
\hline C & -0.04660 & 3.51760 & 14.40840 \\
\hline $\mathrm{H}$ & 0.40310 & 3.15180 & 15.33860 \\
\hline $\mathrm{H}$ & 0.27420 & 2.85460 & 13.59860 \\
\hline $\mathrm{H}$ & -1.13630 & 3.43110 & 14.49870 \\
\hline C & 0.35110 & 4.97360 & 14.15020 \\
\hline C & 3.76230 & 7.91210 & 16.36770 \\
\hline $\mathrm{H}$ & 4.32890 & 8.64590 & 16.95020 \\
\hline $\mathrm{H}$ & 3.05260 & 7.44330 & 17.05710 \\
\hline $\mathrm{H}$ & 3.19930 & 8.45760 & 15.60200 \\
\hline C & 5.38710 & 6.16420 & 16.93310 \\
\hline $\mathrm{H}$ & 5.74710 & 6.88930 & 17.67360 \\
\hline $\mathrm{H}$ & 6.23490 & 5.54390 & 16.63480 \\
\hline $\mathrm{H}$ & 4.64900 & 5.51860 & 17.42460 \\
\hline C & 5.79180 & 7.76380 & 15.0296 \\
\hline $\mathrm{H}$ & 6.19070 & 8.51620 & 15.71980 \\
\hline $\mathrm{H}$ & 5.34020 & 8.28830 & 14.17940 \\
\hline $\mathrm{H}$ & 6.64820 & 7.19530 & 14.65930 \\
\hline 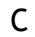 & 4.74630 & 6.90700 & 15.7526 \\
\hline
\end{tabular}


Complex $2 \mathrm{a}$

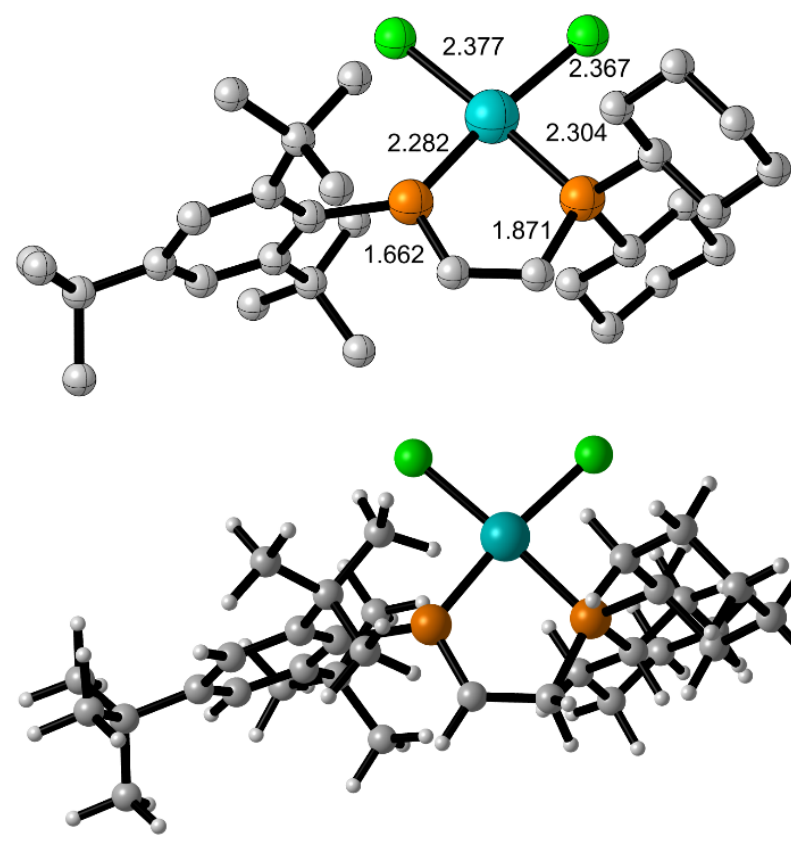

$\mathrm{C}$
$\mathrm{H}$
$\mathrm{H}$
$\mathrm{C}$
$\mathrm{H}$
$\mathrm{C}$
$\mathrm{H}$
$\mathrm{C}$
$\mathrm{C}$
$\mathrm{C}$
$\mathrm{H}$
$\mathrm{C}$
$\mathrm{C}$
$\mathrm{H}$
$\mathrm{C}$
$\mathrm{C}$
$\mathrm{H}$
$\mathrm{H}$
$\mathrm{H}$
$\mathrm{C}$
$\mathrm{H}$

$\begin{array}{lccc}\mathrm{Pd} & 8.40950 & 5.20400 & 13.70060 \\ \mathrm{P} & 9.24640 & 3.27270 & 14.63810 \\ \mathrm{P} & 6.38820 & 4.33140 & 14.30160 \\ \mathrm{Cl} & 10.63810 & 5.91540 & 13.33900 \\ \mathrm{Cl} & 7.42850 & 7.14450 & 12.74150 \\ \mathrm{C} & 7.85940 & 2.35170 & 15.49130 \\ \mathrm{H} & 7.91680 & 1.29020 & 15.21110 \\ \mathrm{H} & 8.04140 & 2.39050 & 16.57480 \\ \mathrm{C} & 6.52120 & 2.92320 & 15.17480 \\ \mathrm{H} & 5.62840 & 2.41170 & 15.53300 \\ \mathrm{C} & 9.90270 & 2.03070 & 13.44130 \\ \mathrm{H} & 10.01370 & 1.09400 & 14.01690 \\ \mathrm{C} & 8.87460 & 1.80530 & 12.33140 \\ \mathrm{H} & 7.90320 & 1.51080 & 12.75090 \\ \mathrm{H} & 8.71580 & 2.76190 & 11.80890 \\ \mathrm{C} & 9.35770 & 0.75760 & 11.33860 \\ \mathrm{H} & 9.43960 & -0.21550 & 11.84720 \\ \mathrm{H} & 8.61420 & 0.63320 & 10.54260 \\ \mathrm{C} & 10.71110 & 1.13880 & 10.75830 \\ \mathrm{H} & 11.05940 & 0.36860 & 10.06100 \\ \mathrm{H} & 10.60550 & 2.06710 & 10.17780 \\ \mathrm{C} & 11.73160 & 1.35830 & 11.86510 \\ \mathrm{H} & 12.69800 & 1.65610 & 11.44380 \\ \mathrm{H} & 11.90010 & 0.40820 & 12.39660 \\ \mathrm{C} & 11.25840 & 2.41630 & 12.85380 \\ \mathrm{H} & 12.00250 & 2.55040 & 13.64840 \\ \mathrm{H} & 11.16880 & 3.38990 & 12.35210 \\ \mathrm{C} & 10.52020 & 3.56520 & 15.92670 \\ \mathrm{H} & 11.35080 & 4.02060 & 15.36510 \\ \mathrm{C} & 11.00780 & 2.29640 & 16.62330 \\ \mathrm{H} & 10.16590 & 1.79770 & 17.12920 \\ \mathrm{H} & 11.40570 & 1.58040 & 15.89320 \\ \mathrm{C} & 12.07340 & 2.63350 & 17.66040 \\ \mathrm{H} & 12.39870 & 1.71970 & 18.17070 \\ \mathrm{H} & 12.95700 & 3.03880 & 17.14550 \\ & & & \\ \mathrm{H} & & \end{array}$

\begin{tabular}{|c|c|c|}
\hline 11.56260 & 3.65830 & 18.66240 \\
\hline 10.72490 & 3.22210 & 19.22910 \\
\hline 12.34270 & 3.89660 & 19.39400 \\
\hline 11.08820 & 4.92140 & 17.95900 \\
\hline 11.93890 & 5.40090 & 17.45270 \\
\hline 10.70630 & 5.64850 & 18.68390 \\
\hline 10.01750 & 4.61200 & 16.92220 \\
\hline 9.73180 & 5.52240 & 16.38120 \\
\hline 9.11210 & 4.24240 & 17.43390 \\
\hline 4.62020 & 4.75230 & 14.13400 \\
\hline 3.88160 & 4.24890 & 13.02520 \\
\hline 2.49960 & 4.36220 & 13.08430 \\
\hline 1.91130 & 3.97300 & 12.26410 \\
\hline 1.82170 & 4.94960 & 14.14430 \\
\hline 2.58200 & 5.49470 & 15.16080 \\
\hline 2.06970 & 5.98760 & 15.97380 \\
\hline 3.97730 & 5.44390 & 15.18890 \\
\hline 5.70610 & 4.29500 & 11.22930 \\
\hline 5.50230 & 5.35770 & 11.06280 \\
\hline 6.00270 & 3.84540 & 10.27470 \\
\hline 6.58710 & 4.24550 & 11.87140 \\
\hline 4.81360 & 2.09840 & 12.08500 \\
\hline 5.57050 & 1.99820 & 12.86620 \\
\hline 5.19670 & 1.60570 & 11.18220 \\
\hline 3.91700 & 1.55830 & 12.41180 \\
\hline 3.47640 & 3.54010 & 10.61080 \\
\hline 3.11750 & 4.54500 & 10.36260 \\
\hline 2.61400 & 2.89290 & 10.79960 \\
\hline 3.97980 & 3.13950 & 9.72540 \\
\hline 4.47790 & 3.56220 & 11.77360 \\
\hline-0.27760 & 5.67680 & 15.36880 \\
\hline 0.00310 & 5.15300 & 16.28990 \\
\hline-1.37140 & 5.67500 & 15.31010 \\
\hline 0.04460 & 6.72100 & 15.45060 \\
\hline-0.17440 & 5.77950 & 12.89990 \\
\hline-1.26960 & 5.83020 & 12.88330 \\
\hline 0.15080 & 5.31360 & 11.96440 \\
\hline 0.21440 & 6.80370 & 12.91670 \\
\hline-0.25190 & 3.56990 & 14.07350 \\
\hline 0.07410 & 2.99270 & 14.94640 \\
\hline 0.07750 & 3.03650 & 13.17620 \\
\hline-1.34820 & 3.58640 & 14.06660 \\
\hline 0.29760 & 4.99830 & 14.12980 \\
\hline 3.79670 & 7.27930 & 16.93500 \\
\hline 4.35910 & 7.80990 & 17.70920 \\
\hline 2.86660 & 6.94550 & 17.40360 \\
\hline 3.55330 & 7.99810 & 16.14520 \\
\hline 4.82400 & 5.11080 & 17.53780 \\
\hline 5.23550 & 5.61090 & 18.42310 \\
\hline 5.50610 & 4.29840 & 17.26610 \\
\hline 3.85950 & 4.66970 & 17.81530 \\
\hline 6.01800 & 6.75830 & 16.07700 \\
\hline 6.30190 & 7.43420 & 16.89080 \\
\hline 6.00320 & 7.33630 & 15.14680 \\
\hline 6.83620 & 6.03550 & 16.00540 \\
\hline 4.66090 & 6.12550 & 16.40010 \\
\hline
\end{tabular}




\section{Experimental Details for Crystal Structure Refinement and Acquisition}

Data for all structures were collected at $100 \mathrm{~K}$ using a Bruker Ultra diffractometer equipped with micro-focus optics. Samples were mounted on nylon loops with Paratone$\mathrm{N}$ oil. Standard data reduction and refinement procedures were employed using APEX II, SHELXTL and OLEX2 software. In $\mathbf{2 a}$, there are two fractionally occupied (58 and $75 \%$ refined occupancies) and disordered molecules of $\mathrm{CHCl}_{3}$. All six independent $\mathrm{C}-\mathrm{Cl}$ bond distances were collectively refined to $1.73 \AA$. All hydrogen atoms were treated as idealized isotropic contributions and all non-hydrogen atoms were refined with anisotropic thermal parameters.

\section{Data for $E-1$ a}

Table S1. Crystal data and structure refinement for cain02 $(\boldsymbol{E}-\mathbf{1 a})$.

Identification code

Empirical formula

Formula weight

Temperature

Wavelength

Crystal system

Space group

Unit cell dimensions

Volume

$\mathrm{Z}$

Density (calculated)

Absorption coefficient

$\mathrm{F}(000)$

Crystal size

Theta range for data collection

Index ranges

Reflections collected

Independent reflections

Completeness to theta $=25.000^{\circ}$

Absorption correction

Refinement method

Data / restraints / parameters

Goodness-of-fit on $\mathrm{F}^{2}$

Final R indices [I $>2 \operatorname{sigma}(\mathrm{I})]$ cain02

C32 H54 P2

500.69

100(2) K

$0.71073 \AA$

Triclinic

$\mathrm{P}-1$

$\mathrm{a}=9.8594(12) \AA \quad \alpha=101.584(4)^{\circ}$.

$\mathrm{b}=11.7510(16) \AA \quad \beta=109.156(4)^{\circ}$.

$\mathrm{c}=14.647(2) \AA \quad \gamma=99.100(5)^{\circ}$.

1523.6(4) $\AA^{3}$

2

$1.091 \mathrm{Mg} / \mathrm{m}^{3}$

$0.160 \mathrm{~mm}^{-1}$

552

$0.30 \times 0.27 \times 0.22 \mathrm{~mm}^{3}$

1.529 to $28.325^{\circ}$.

$-13<=\mathrm{h}<=12,-15<=\mathrm{k}<=15,0<=\mathrm{l}<=19$

7168

$7168[\mathrm{R}(\mathrm{int})=0.045]$

$99.1 \%$

Multi-scan

Full-matrix least-squares on $\mathrm{F}^{2}$

7168 / 0 / 336

1.057

$\mathrm{R} 1=0.0412, \mathrm{wR} 2=0.1043$ 
$\mathrm{R}$ indices (all data)

Extinction coefficient

Largest diff. peak and hole
$\mathrm{R} 1=0.0510, \mathrm{wR} 2=0.1116$

$\mathrm{n} / \mathrm{a}$

0.410 and -0.256 e. $\AA^{-3}$ 
Table S2. Atomic coordinates ( x 10 $0^{4}$ ) and equivalent isotropic displacement parameters $\left(\AA^{2} \times 10^{3}\right)$ for cain02. $U(e q)$ is defined as one third of the trace of the orthogonalized $U^{i j}$ tensor.

\begin{tabular}{|c|c|c|c|c|}
\hline & $\mathrm{x}$ & $\mathrm{y}$ & $\mathrm{z}$ & $\mathrm{U}(\mathrm{eq})$ \\
\hline $\mathrm{P}(1)$ & $324(1)$ & 2934(1) & $5417(1)$ & $16(1)$ \\
\hline $\mathrm{P}(2)$ & $32(1)$ & $931(1)$ & $7468(1)$ & $17(1)$ \\
\hline $\mathrm{C}(1)$ & $-793(2)$ & 1771(1) & $5758(1)$ & $18(1)$ \\
\hline $\mathrm{C}(2)$ & $-332(2)$ & 1981(1) & $6875(1)$ & $17(1)$ \\
\hline $\mathrm{C}(3)$ & $1584(2)$ & $2126(1)$ & 4994(1) & $18(1)$ \\
\hline $\mathrm{C}(4)$ & $2590(2)$ & $2998(2)$ & $4699(2)$ & $30(1)$ \\
\hline $\mathrm{C}(5)$ & $3734(2)$ & $2441(2)$ & $4397(2)$ & $31(1)$ \\
\hline $\mathrm{C}(6)$ & $4659(2)$ & 1962(2) & $5218(1)$ & $27(1)$ \\
\hline$C(7)$ & $3662(2)$ & $1075(2)$ & $5495(1)$ & $26(1)$ \\
\hline $\mathrm{C}(8)$ & $2530(2)$ & $1639(2)$ & $5807(1)$ & $23(1)$ \\
\hline $\mathrm{C}(9)$ & $-1054(2)$ & 2971(1) & $4201(1)$ & $18(1)$ \\
\hline $\mathrm{C}(10)$ & $-2133(2)$ & $3696(2)$ & $4413(1)$ & $21(1)$ \\
\hline $\mathrm{C}(11)$ & $-3239(2)$ & $3829(2)$ & $3446(1)$ & $24(1)$ \\
\hline $\mathrm{C}(12)$ & $-4070(2)$ & $2607(2)$ & $2705(1)$ & $24(1)$ \\
\hline $\mathrm{C}(13)$ & $-3001(2)$ & 1907(2) & $2467(1)$ & $25(1)$ \\
\hline $\mathrm{C}(14)$ & $-1896(2)$ & $1759(2)$ & $3429(1)$ & $23(1)$ \\
\hline $\mathrm{C}(15)$ & $519(2)$ & 1774(1) & $8795(1)$ & $16(1)$ \\
\hline$C(16)$ & $-552(2)$ & $1672(1)$ & $9247(1)$ & $16(1)$ \\
\hline $\mathrm{C}(17)$ & $-271(2)$ & $2510(1)$ & 10157(1) & $17(1)$ \\
\hline $\mathrm{C}(18)$ & $1028(2)$ & $3407(1)$ & $10647(1)$ & $17(1)$ \\
\hline $\mathrm{C}(19)$ & $2105(2)$ & $3406(1)$ & $10236(1)$ & $19(1)$ \\
\hline $\mathrm{C}(20)$ & 1924(2) & $2600(1)$ & $9340(1)$ & $18(1)$ \\
\hline $\mathrm{C}(21)$ & $-1999(2)$ & $664(1)$ & $8808(1)$ & $18(1)$ \\
\hline $\mathrm{C}(22)$ & $-3136(2)$ & $847(2)$ & 7871(1) & $22(1)$ \\
\hline $\mathrm{C}(23)$ & $-1663(2)$ & $-573(1)$ & $8560(1)$ & $22(1)$ \\
\hline$C(24)$ & $-2756(2)$ & $615(2)$ & $9574(1)$ & $26(1)$ \\
\hline$C(25)$ & $1328(2)$ & $4348(2)$ & 11632(1) & $23(1)$ \\
\hline$C(26)$ & $93(3)$ & $4197(2)$ & $12013(2)$ & $32(1)$ \\
\hline$C\left(26^{\prime}\right)$ & $-503(16)$ & $4600(13)$ & $11473(11)$ & $34(4)$ \\
\hline $\mathrm{C}(27)$ & 2762(3) & $4251(2)$ & $12456(2)$ & $30(1)$ \\
\hline $\mathrm{C}\left(27^{\prime}\right)$ & $1480(30)$ & $3746(18)$ & $12419(12)$ & $58(7)$ \\
\hline
\end{tabular}




\begin{tabular}{lllcl}
$\mathrm{C}(28)$ & $1649(4)$ & $5614(4)$ & $11485(3)$ & $26(1)$ \\
$\mathrm{C}\left(28^{\prime}\right)$ & $2050(40)$ & $5430(20)$ & $11730(20)$ & $40(6)$ \\
$\mathrm{C}(29)$ & $3281(2)$ & $2685(2)$ & $9022(1)$ & $21(1)$ \\
$\mathrm{C}(30)$ & $4736(2)$ & $3141(2)$ & $9947(1)$ & $34(1)$ \\
$\mathrm{C}(31)$ & $3254(2)$ & $3577(2)$ & $8379(1)$ & $28(1)$ \\
$\mathrm{C}(32)$ & $3400(2)$ & $1466(2)$ & $8468(1)$ & $24(1)$ \\
\hline
\end{tabular}


Table S3. Bond lengths $[\AA ̊]$ and angles $\left[{ }^{\circ}\right]$ for cain 02 .

\begin{tabular}{|c|c|}
\hline $\mathrm{P}(1)-\mathrm{C}(3)$ & $1.8633(16)$ \\
\hline $\mathrm{P}(1)-\mathrm{C}(9)$ & $1.8653(16)$ \\
\hline $\mathrm{P}(1)-\mathrm{C}(1)$ & $1.8685(16)$ \\
\hline $\mathrm{P}(2)-\mathrm{C}(2)$ & $1.6645(17)$ \\
\hline$P(2)-C(15)$ & $1.8575(15)$ \\
\hline$C(1)-C(2)$ & $1.502(2)$ \\
\hline $\mathrm{C}(3)-\mathrm{C}(8)$ & $1.526(2)$ \\
\hline$C(3)-C(4)$ & $1.536(2)$ \\
\hline$C(4)-C(5)$ & $1.531(2)$ \\
\hline $\mathrm{C}(5)-\mathrm{C}(6)$ & $1.519(3)$ \\
\hline$C(6)-C(7)$ & $1.525(3)$ \\
\hline $\mathrm{C}(7)-\mathrm{C}(8)$ & $1.530(2)$ \\
\hline $\mathrm{C}(9)-\mathrm{C}(10)$ & $1.534(2)$ \\
\hline $\mathrm{C}(9)-\mathrm{C}(14)$ & $1.535(2)$ \\
\hline $\mathrm{C}(10)-\mathrm{C}(11)$ & $1.532(2)$ \\
\hline $\mathrm{C}(11)-\mathrm{C}(12)$ & $1.526(2)$ \\
\hline $\mathrm{C}(12)-\mathrm{C}(13)$ & $1.519(2)$ \\
\hline C(13)-C(14) & $1.534(2)$ \\
\hline $\mathrm{C}(15)-\mathrm{C}(16)$ & $1.421(2)$ \\
\hline$C(15)-C(20)$ & $1.427(2)$ \\
\hline $\mathrm{C}(16)-\mathrm{C}(17)$ & $1.402(2)$ \\
\hline$C(16)-C(21)$ & $1.552(2)$ \\
\hline $\mathrm{C}(17)-\mathrm{C}(18)$ & $1.390(2)$ \\
\hline C(18)-C(19) & $1.381(2)$ \\
\hline$C(18)-C(25)$ & $1.539(2)$ \\
\hline C(19)-C(20) & $1.395(2)$ \\
\hline C(20)-C(29) & $1.551(2)$ \\
\hline $\mathrm{C}(21)-\mathrm{C}(22)$ & $1.540(2)$ \\
\hline $\mathrm{C}(21)-\mathrm{C}(23)$ & $1.541(2)$ \\
\hline $\mathrm{C}(21)-\mathrm{C}(24)$ & $1.543(2)$ \\
\hline $\mathrm{C}(25)-\mathrm{C}\left(28^{\prime}\right)$ & $1.31(3)$ \\
\hline $\mathrm{C}(25)-\mathrm{C}\left(27^{\prime}\right)$ & $1.449(17)$ \\
\hline$C(25)-C(26)$ & $1.498(3)$ \\
\hline $\mathrm{C}(25)-\mathrm{C}(28)$ & $1.544(4)$ \\
\hline
\end{tabular}




\begin{tabular}{|c|c|}
\hline$C(25)-C(27)$ & $1.567(3)$ \\
\hline$C(25)-C\left(26^{\prime}\right)$ & $1.822(14)$ \\
\hline$C(29)-C(31)$ & $1.540(3)$ \\
\hline $\mathrm{C}(29)-\mathrm{C}(30)$ & $1.540(2)$ \\
\hline $\mathrm{C}(29)-\mathrm{C}(32)$ & $1.540(2)$ \\
\hline $\mathrm{C}(3)-\mathrm{P}(1)-\mathrm{C}(9)$ & $102.00(7)$ \\
\hline $\mathrm{C}(3)-\mathrm{P}(1)-\mathrm{C}(1)$ & $102.90(7)$ \\
\hline $\mathrm{C}(9)-\mathrm{P}(1)-\mathrm{C}(1)$ & $100.84(7)$ \\
\hline $\mathrm{C}(2)-\mathrm{P}(2)-\mathrm{C}(15)$ & $101.59(7)$ \\
\hline $\mathrm{C}(2)-\mathrm{C}(1)-\mathrm{P}(1)$ & $112.24(11)$ \\
\hline $\mathrm{C}(1)-\mathrm{C}(2)-\mathrm{P}(2)$ & $123.88(12)$ \\
\hline $\mathrm{C}(8)-\mathrm{C}(3)-\mathrm{C}(4)$ & 109.62(13) \\
\hline $\mathrm{C}(8)-\mathrm{C}(3)-\mathrm{P}(1)$ & $112.29(11)$ \\
\hline $\mathrm{C}(4)-\mathrm{C}(3)-\mathrm{P}(1)$ & $108.24(11)$ \\
\hline$C(5)-C(4)-C(3)$ & $112.42(15)$ \\
\hline$C(6)-C(5)-C(4)$ & $111.60(16)$ \\
\hline$C(5)-C(6)-C(7)$ & $110.32(14)$ \\
\hline$C(6)-C(7)-C(8)$ & $111.34(15)$ \\
\hline $\mathrm{C}(3)-\mathrm{C}(8)-\mathrm{C}(7)$ & $112.28(14)$ \\
\hline $\mathrm{C}(10)-\mathrm{C}(9)-\mathrm{C}(14)$ & $110.78(13)$ \\
\hline $\mathrm{C}(10)-\mathrm{C}(9)-\mathrm{P}(1)$ & 109.13(10) \\
\hline $\mathrm{C}(14)-\mathrm{C}(9)-\mathrm{P}(1)$ & $116.79(12)$ \\
\hline $\mathrm{C}(11)-\mathrm{C}(10)-\mathrm{C}(9)$ & $111.90(13)$ \\
\hline $\mathrm{C}(12)-\mathrm{C}(11)-\mathrm{C}(10)$ & $110.94(14)$ \\
\hline $\mathrm{C}(13)-\mathrm{C}(12)-\mathrm{C}(11)$ & $110.95(14)$ \\
\hline $\mathrm{C}(12)-\mathrm{C}(13)-\mathrm{C}(14)$ & $110.84(14)$ \\
\hline $\mathrm{C}(13)-\mathrm{C}(14)-\mathrm{C}(9)$ & $111.84(14)$ \\
\hline$C(16)-C(15)-C(20)$ & $119.38(13)$ \\
\hline $\mathrm{C}(16)-\mathrm{C}(15)-\mathrm{P}(2)$ & $120.03(11)$ \\
\hline $\mathrm{C}(20)-\mathrm{C}(15)-\mathrm{P}(2)$ & $120.38(11)$ \\
\hline$C(17)-C(16)-C(15)$ & $118.32(14)$ \\
\hline$C(17)-C(16)-C(21)$ & $117.92(14)$ \\
\hline$C(15)-C(16)-C(21)$ & $123.74(13)$ \\
\hline $\mathrm{C}(18)-\mathrm{C}(17)-\mathrm{C}(16)$ & $122.74(14)$ \\
\hline $\mathrm{C}(19)-\mathrm{C}(18)-\mathrm{C}(17)$ & $117.28(14)$ \\
\hline
\end{tabular}




$\begin{array}{lc}\mathrm{C}(19)-\mathrm{C}(18)-\mathrm{C}(25) & 119.67(14) \\ \mathrm{C}(17)-\mathrm{C}(18)-\mathrm{C}(25) & 123.00(14) \\ \mathrm{C}(18)-\mathrm{C}(19)-\mathrm{C}(20) & 123.67(15) \\ \mathrm{C}(19)-\mathrm{C}(20)-\mathrm{C}(15) & 117.82(14) \\ \mathrm{C}(19)-\mathrm{C}(20)-\mathrm{C}(29) & 116.63(13) \\ \mathrm{C}(15)-\mathrm{C}(20)-\mathrm{C}(29) & 125.55(13) \\ \mathrm{C}(22)-\mathrm{C}(21)-\mathrm{C}(23) & 110.33(13) \\ \mathrm{C}(22)-\mathrm{C}(21)-\mathrm{C}(24) & 106.85(13) \\ \mathrm{C}(23)-\mathrm{C}(21)-\mathrm{C}(24) & 105.26(14) \\ \mathrm{C}(22)-\mathrm{C}(21)-\mathrm{C}(16) & 111.83(13) \\ \mathrm{C}(23)-\mathrm{C}(21)-\mathrm{C}(16) & 110.63(12) \\ \mathrm{C}(24)-\mathrm{C}(21)-\mathrm{C}(16) & 111.69(13) \\ \mathrm{C}\left(28^{\prime}\right)-\mathrm{C}(25)-\mathrm{C}\left(27^{\prime}\right) & 126.3(15) \\ \mathrm{C}\left(28^{\prime}\right)-\mathrm{C}(25)-\mathrm{C}(18) & 116.2(11) \\ \mathrm{C}\left(27^{\prime}\right)-\mathrm{C}(25)-\mathrm{C}(18) & 108.3(7) \\ \mathrm{C}(26)-\mathrm{C}(25)-\mathrm{C}(18) & 113.54(15) \\ \mathrm{C}(26)-\mathrm{C}(25)-\mathrm{C}(28) & 110.1(2) \\ \mathrm{C}(18)-\mathrm{C}(25)-\mathrm{C}(28) & 109.39(19) \\ \mathrm{C}(26)-\mathrm{C}(25)-\mathrm{C}(27) & 108.14(17) \\ \mathrm{C}(18)-\mathrm{C}(25)-\mathrm{C}(27) & 108.64(14) \\ \mathrm{C}(28)-\mathrm{C}(25)-\mathrm{C}(27) & 106.81(19) \\ \mathrm{C}\left(28^{\prime}\right)-\mathrm{C}(25)-\mathrm{C}\left(26^{\prime}\right) & 100.3(13) \\ \mathrm{C}\left(27^{\prime}\right)-\mathrm{C}(25)-\mathrm{C}\left(26^{\prime}\right) & 97.8(11) \\ \mathrm{C}(18)-\mathrm{C}(25)-\mathrm{C}\left(26^{\prime}\right) & 102.4(4) \\ \mathrm{C}(31)-\mathrm{C}(29)-\mathrm{C}(30) & 107.61(15) \\ \mathrm{C}(31)-\mathrm{C}(29)-\mathrm{C}(32) & 110.69(14) \\ \mathrm{C}(30)-\mathrm{C}(29)-\mathrm{C}(32) & 104.07(14) \\ \mathrm{C}(31)-\mathrm{C}(29)-\mathrm{C}(20) & 109.91(14) \\ \mathrm{C}(30)-\mathrm{C}(29)-\mathrm{C}(20) & 110.89(13) \\ \mathrm{C}(32)-\mathrm{C}(29)-\mathrm{C}(20) & 113.39(13) \\ & \end{array}$


Table S4. Anisotropic displacement parameters $\left(\AA^{2} \times 10^{3}\right)$ for cain02. The anisotropic displacement factor exponent takes the form: $-2 \pi^{2}\left[h^{2} a^{* 2} U^{11}+\ldots+2 h \mathrm{k}^{*} b^{*} U^{12}\right]$

\begin{tabular}{|c|c|c|c|c|c|c|}
\hline & $\mathrm{U}^{11}$ & $\mathrm{U}^{22}$ & $\mathrm{U}^{33}$ & $\mathrm{U}^{23}$ & $\mathrm{U}^{13}$ & $\mathrm{U}^{12}$ \\
\hline $\mathrm{P}(1)$ & $15(1)$ & $17(1)$ & $16(1)$ & $4(1)$ & $7(1)$ & $3(1)$ \\
\hline $\mathrm{P}(2)$ & $19(1)$ & $16(1)$ & $16(1)$ & $2(1)$ & $7(1)$ & $3(1)$ \\
\hline $\mathrm{C}(1)$ & $16(1)$ & $21(1)$ & 19(1) & $6(1)$ & $8(1)$ & $3(1)$ \\
\hline $\mathrm{C}(2)$ & $16(1)$ & $19(1)$ & 19(1) & $4(1)$ & $9(1)$ & $4(1)$ \\
\hline $\mathrm{C}(3)$ & $17(1)$ & $19(1)$ & 19(1) & $4(1)$ & $8(1)$ & $5(1)$ \\
\hline $\mathrm{C}(4)$ & $31(1)$ & $32(1)$ & $44(1)$ & $22(1)$ & $26(1)$ & $16(1)$ \\
\hline $\mathrm{C}(5)$ & $30(1)$ & $39(1)$ & $41(1)$ & $20(1)$ & $24(1)$ & $16(1)$ \\
\hline$C(6)$ & $17(1)$ & $30(1)$ & $35(1)$ & $6(1)$ & $12(1)$ & $5(1)$ \\
\hline $\mathrm{C}(7)$ & $22(1)$ & $29(1)$ & $32(1)$ & $13(1)$ & 11(1) & $12(1)$ \\
\hline $\mathrm{C}(8)$ & $21(1)$ & $29(1)$ & $23(1)$ & $9(1)$ & $10(1)$ & $9(1)$ \\
\hline $\mathrm{C}(9)$ & $16(1)$ & $20(1)$ & $17(1)$ & $5(1)$ & $7(1)$ & $4(1)$ \\
\hline $\mathrm{C}(10)$ & $19(1)$ & $19(1)$ & $22(1)$ & $3(1)$ & $6(1)$ & $5(1)$ \\
\hline $\mathrm{C}(11)$ & $21(1)$ & $21(1)$ & $27(1)$ & $7(1)$ & $3(1)$ & $6(1)$ \\
\hline $\mathrm{C}(12)$ & $20(1)$ & $25(1)$ & $21(1)$ & $6(1)$ & $4(1)$ & $3(1)$ \\
\hline$C(13)$ & $23(1)$ & $29(1)$ & 19(1) & $2(1)$ & $6(1)$ & $5(1)$ \\
\hline$C(14)$ & $23(1)$ & $23(1)$ & $21(1)$ & $2(1)$ & $6(1)$ & $8(1)$ \\
\hline$C(15)$ & $18(1)$ & $13(1)$ & $17(1)$ & $4(1)$ & $7(1)$ & $5(1)$ \\
\hline$C(16)$ & $16(1)$ & $16(1)$ & 19(1) & $9(1)$ & $6(1)$ & $7(1)$ \\
\hline$C(17)$ & $21(1)$ & $18(1)$ & 19(1) & $9(1)$ & $10(1)$ & $9(1)$ \\
\hline $\mathrm{C}(18)$ & $24(1)$ & $15(1)$ & $17(1)$ & $7(1)$ & $9(1)$ & $7(1)$ \\
\hline$C(19)$ & $21(1)$ & $15(1)$ & 19(1) & $3(1)$ & $7(1)$ & 1(1) \\
\hline $\mathrm{C}(20)$ & $18(1)$ & $16(1)$ & $18(1)$ & $5(1)$ & $7(1)$ & $3(1)$ \\
\hline$C(21)$ & $15(1)$ & $21(1)$ & $20(1)$ & $9(1)$ & $5(1)$ & $4(1)$ \\
\hline$C(22)$ & $16(1)$ & $28(1)$ & $24(1)$ & $11(1)$ & $7(1)$ & $7(1)$ \\
\hline$C(23)$ & $19(1)$ & $18(1)$ & $28(1)$ & $8(1)$ & $5(1)$ & $2(1)$ \\
\hline$C(24)$ & $21(1)$ & $32(1)$ & $25(1)$ & 11(1) & $10(1)$ & $2(1)$ \\
\hline$C(25)$ & $35(1)$ & $17(1)$ & 19(1) & $4(1)$ & $13(1)$ & $8(1)$ \\
\hline$C(26)$ & $37(1)$ & $30(1)$ & $29(1)$ & $-1(1)$ & $21(1)$ & $2(1)$ \\
\hline$C\left(26^{\prime}\right)$ & $37(8)$ & $30(8)$ & $33(8)$ & $-12(6)$ & $18(6)$ & $13(6)$ \\
\hline$C(27)$ & $39(1)$ & $30(1)$ & $18(1)$ & $5(1)$ & $4(1)$ & $15(1)$ \\
\hline $\mathrm{C}\left(27^{\prime}\right)$ & 107(19) & $57(12)$ & $27(8)$ & $17(8)$ & $24(9)$ & $58(12)$ \\
\hline
\end{tabular}




\begin{tabular}{lllllll}
$\mathrm{C}(28)$ & $43(2)$ & $14(1)$ & $26(2)$ & $6(1)$ & $15(1)$ & $9(1)$ \\
$\mathrm{C}\left(28^{\prime}\right)$ & $77(19)$ & $23(11)$ & $38(14)$ & $6(9)$ & $43(14)$ & $18(10)$ \\
$\mathrm{C}(29)$ & $17(1)$ & $21(1)$ & $21(1)$ & $0(1)$ & $8(1)$ & $0(1)$ \\
$\mathrm{C}(30)$ & $19(1)$ & $46(1)$ & $27(1)$ & $-4(1)$ & $8(1)$ & $2(1)$ \\
$\mathrm{C}(31)$ & $27(1)$ & $25(1)$ & $31(1)$ & $5(1)$ & $17(1)$ & $-1(1)$ \\
$\mathrm{C}(32)$ & $19(1)$ & $26(1)$ & $26(1)$ & $3(1)$ & $10(1)$ & $7(1)$ \\
\hline
\end{tabular}


Table S5. Hydrogen coordinates ( $\left.\mathrm{x} 10^{4}\right)$ and isotropic displacement parameters $\left(\AA^{2} \mathrm{x} 10^{3}\right)$ for cain02.

\begin{tabular}{|c|c|c|c|c|}
\hline & $\mathrm{x}$ & $\mathrm{y}$ & $\mathrm{z}$ & $\mathrm{U}(\mathrm{eq})$ \\
\hline $\mathrm{H}(1 \mathrm{~A})$ & -1851 & 1771 & 5466 & 22 \\
\hline $\mathrm{H}(1 \mathrm{~B})$ & -679 & 973 & 5465 & 22 \\
\hline $\mathrm{H}(2 \mathrm{~A})$ & -244 & 2759 & 7265 & 21 \\
\hline $\mathrm{H}(3 \mathrm{~A})$ & 976 & 1443 & 4391 & 22 \\
\hline $\mathrm{H}(4 \mathrm{~A})$ & 1972 & 3255 & 4131 & 36 \\
\hline $\mathrm{H}(4 \mathrm{~B})$ & 3110 & 3719 & 5273 & 36 \\
\hline $\mathrm{H}(5 \mathrm{~A})$ & 3219 & 1779 & 3774 & 38 \\
\hline $\mathrm{H}(5 \mathrm{~B})$ & 4393 & 3052 & 4256 & 38 \\
\hline $\mathrm{H}(6 \mathrm{~A})$ & 5263 & 2634 & 5818 & 32 \\
\hline $\mathrm{H}(6 \mathrm{~B})$ & 5340 & 1560 & 4982 & 32 \\
\hline $\mathrm{H}(7 \mathrm{~A})$ & 4277 & 805 & 6055 & 31 \\
\hline $\mathrm{H}(7 \mathrm{~B})$ & 3136 & 365 & 4913 & 31 \\
\hline $\mathrm{H}(8 \mathrm{~A})$ & 1879 & 1031 & 5956 & 28 \\
\hline $\mathrm{H}(8 \mathrm{~B})$ & 3057 & 2300 & 6429 & 28 \\
\hline $\mathrm{H}(9 \mathrm{~A})$ & -490 & 3430 & 3880 & 21 \\
\hline $\mathrm{H}(10 \mathrm{~A})$ & -2680 & 3293 & 4764 & 25 \\
\hline $\mathrm{H}(10 \mathrm{~B})$ & -1566 & 4501 & 4861 & 25 \\
\hline $\mathrm{H}(11 \mathrm{~A})$ & -3955 & 4254 & 3609 & 29 \\
\hline $\mathrm{H}(11 \mathrm{~B})$ & -2705 & 4314 & 3133 & 29 \\
\hline $\mathrm{H}(12 \mathrm{~A})$ & -4673 & 2148 & 2994 & 28 \\
\hline $\mathrm{H}(12 \mathrm{~B})$ & -4748 & 2716 & 2077 & 28 \\
\hline $\mathrm{H}(13 \mathrm{~A})$ & -2456 & 2333 & 2126 & 30 \\
\hline $\mathrm{H}(13 \mathrm{~B})$ & -3562 & 1108 & 2006 & 30 \\
\hline $\mathrm{H}(14 \mathrm{~A})$ & -1180 & 1340 & 3259 & 28 \\
\hline $\mathrm{H}(14 \mathrm{~B})$ & -2435 & 1258 & 3730 & 28 \\
\hline $\mathrm{H}(17 \mathrm{~A})$ & -999 & 2463 & 10451 & 21 \\
\hline $\mathrm{H}(19 \mathrm{~A})$ & 3020 & 3986 & 10584 & 23 \\
\hline $\mathrm{H}(22 \mathrm{~A})$ & -4032 & 196 & 7615 & 33 \\
\hline $\mathrm{H}(22 \mathrm{~B})$ & -2713 & 848 & 7352 & 33 \\
\hline $\mathrm{H}(22 \mathrm{C})$ & -3387 & 1615 & 8049 & 33 \\
\hline
\end{tabular}




\begin{tabular}{|c|c|c|c|c|}
\hline $\mathrm{H}(23 \mathrm{~A})$ & -2588 & -1197 & 8284 & 34 \\
\hline $\mathrm{H}(23 \mathrm{~B})$ & -997 & -703 & 9173 & 34 \\
\hline $\mathrm{H}(23 \mathrm{C})$ & -1189 & -606 & 8066 & 34 \\
\hline $\mathrm{H}(24 \mathrm{~A})$ & -3663 & -29 & 9272 & 39 \\
\hline $\mathrm{H}(24 \mathrm{~B})$ & -3002 & 1381 & 9764 & 39 \\
\hline $\mathrm{H}(24 \mathrm{C})$ & -2083 & 461 & 10172 & 39 \\
\hline $\mathrm{H}(26 \mathrm{~A})$ & -98 & 3393 & 12108 & 48 \\
\hline $\mathrm{H}(26 \mathrm{~B})$ & -802 & 4301 & 11525 & 48 \\
\hline $\mathrm{H}(26 \mathrm{C})$ & 371 & 4797 & 12655 & 48 \\
\hline $\mathrm{H}(26 \mathrm{D})$ & -1180 & 3849 & 11392 & 52 \\
\hline $\mathrm{H}(26 \mathrm{E})$ & -871 & 4888 & 10876 & 52 \\
\hline $\mathrm{H}(26 \mathrm{~F})$ & -441 & 5200 & 12066 & 52 \\
\hline $\mathrm{H}(27 \mathrm{~A})$ & 3585 & 4348 & 12221 & 45 \\
\hline $\mathrm{H}(27 \mathrm{~B})$ & 2595 & 3466 & 12587 & 45 \\
\hline $\mathrm{H}(27 \mathrm{C})$ & 3002 & 4881 & 13076 & 45 \\
\hline $\mathrm{H}(27 \mathrm{D})$ & 810 & 2944 & 12138 & 87 \\
\hline $\mathrm{H}(27 \mathrm{E})$ & 1219 & 4207 & 12947 & 87 \\
\hline $\mathrm{H}(27 \mathrm{~F})$ & 2500 & 3680 & 12704 & 87 \\
\hline $\mathrm{H}(28 \mathrm{~A})$ & 2462 & 5706 & 11239 & 40 \\
\hline $\mathrm{H}(28 \mathrm{~B})$ & 1928 & 6214 & 12127 & 40 \\
\hline $\mathrm{H}(28 \mathrm{C})$ & 761 & 5725 & 10994 & 40 \\
\hline $\mathrm{H}(28 \mathrm{D})$ & 1701 & 5627 & 11091 & 61 \\
\hline $\mathrm{H}(28 \mathrm{E})$ & 3110 & 5459 & 11937 & 61 \\
\hline $\mathrm{H}(28 \mathrm{~F})$ & 1882 & 6001 & 12245 & 61 \\
\hline $\mathrm{H}(30 \mathrm{~A})$ & 4752 & 3923 & 10347 & 51 \\
\hline $\mathrm{H}(30 \mathrm{~B})$ & 5576 & 3219 & 9728 & 51 \\
\hline $\mathrm{H}(30 \mathrm{C})$ & 4805 & 2571 & 10356 & 51 \\
\hline $\mathrm{H}(31 \mathrm{~A})$ & 3177 & 4345 & 8743 & 41 \\
\hline $\mathrm{H}(31 \mathrm{~B})$ & 2401 & 3260 & 7744 & 41 \\
\hline $\mathrm{H}(31 \mathrm{C})$ & 4168 & 3692 & 8242 & 41 \\
\hline $\mathrm{H}(32 \mathrm{~A})$ & 4275 & 1579 & 8287 & 36 \\
\hline $\mathrm{H}(32 \mathrm{~B})$ & 2512 & 1112 & 7858 & 36 \\
\hline $\mathrm{H}(32 \mathrm{C})$ & 3488 & 930 & 8907 & 36 \\
\hline
\end{tabular}




\section{Data for $E-1 b$}

Table S6. Crystal data and structure refinement for cain09_a $(\boldsymbol{E}-\mathbf{1 b})$.

Identification code

cain09_a

Empirical formula

C32 H42 P2

Formula weight

488.59

Temperature

$100.0 \mathrm{~K}$

Wavelength

$0.71073 \AA$

Crystal system

Triclinic

Space group

P-1

Unit cell dimensions

$\mathrm{a}=10.4449(13) \AA$

$\mathrm{a}=101.749(3)^{\circ}$.

$\mathrm{b}=14.5401(16) \AA$

$b=98.922(4)^{\circ}$.

$\mathrm{c}=21.135(2) \AA$

$\mathrm{g}=110.134(4)^{\circ}$.

Volume

$2860.5(6) \AA^{3}$

$\mathrm{Z}$

4

Density (calculated)

$1.135 \mathrm{Mg} / \mathrm{m}^{3}$

Absorption coefficient

$0.170 \mathrm{~mm}^{-1}$

$\mathrm{F}(000)$

1056

Crystal size

$0.28 \times 0.2 \times 0.1 \mathrm{~mm}^{3}$

Theta range for data collection

1.602 to $25.396^{\circ}$.

Index ranges

$-10<=\mathrm{h}<=12,-17<=\mathrm{k}<=15,-25<=1<=25$

Reflections collected

32571

Independent reflections

$10426[\mathrm{R}(\mathrm{int})=0.0429]$

Completeness to theta $=25.396^{\circ}$

$98.8 \%$

Absorption correction

Semi-empirical from equivalents

Max. and min. transmission

0.0916 and 0.0671

Refinement method

Full-matrix least-squares on $\mathrm{F}^{2}$

Data / restraints / parameters

10426 / 0 / 639

Goodness-of-fit on $\mathrm{F}^{2}$

1.029

Final $\mathrm{R}$ indices [I $>2 \operatorname{sigma}(\mathrm{I})]$

$\mathrm{R} 1=0.0495, \mathrm{wR} 2=0.1132$

$\mathrm{R}$ indices (all data)

$\mathrm{R} 1=0.0760, \mathrm{wR} 2=0.1274$

Extinction coefficient

$\mathrm{n} / \mathrm{a}$

Largest diff. peak and hole

0.342 and -0.281 e. $\AA^{-3}$ 
Table S7. Atomic coordinates ( $\left.\mathrm{x} 10^{4}\right)$ and equivalent isotropic displacement parameters $\left(\AA^{2} \times 10^{3}\right)$ for cain09_a. U(eq) is defined as one third of the trace of the orthogonalized $U^{\mathrm{ij}}$ tensor.

\begin{tabular}{|c|c|c|c|c|}
\hline & $\mathrm{x}$ & $\mathrm{y}$ & $\mathrm{z}$ & $\mathrm{U}(\mathrm{eq})$ \\
\hline $\mathrm{P}(3)$ & 7231(1) & 6703(1) & $6374(1)$ & $27(1)$ \\
\hline $\mathrm{P}(4)$ & $4812(1)$ & 6402(1) & $7670(1)$ & $32(1)$ \\
\hline $\mathrm{P}(2)$ & $4549(1)$ & $6436(1)$ & $2656(1)$ & $31(1)$ \\
\hline $\mathrm{P}(1)$ & $2348(1)$ & $5322(1)$ & $802(1)$ & $30(1)$ \\
\hline$C(19)$ & $5257(3)$ & $9476(2)$ & $3400(1)$ & $30(1)$ \\
\hline $\mathrm{C}(48)$ & $5185(3)$ & $8463(2)$ & $8225(1)$ & $28(1)$ \\
\hline $\mathrm{C}(18)$ & $6700(2)$ & 9972(2) & $3650(1)$ & $27(1)$ \\
\hline $\mathrm{C}(49)$ & $4647(3)$ & $9225(2)$ & $8346(1)$ & $28(1)$ \\
\hline$C(15)$ & $5396(3)$ & $7824(2)$ & $3079(1)$ & $27(1)$ \\
\hline$C(52)$ & 2753(3) & 7204(2) & $7955(1)$ & $26(1)$ \\
\hline $\mathrm{C}(53)$ & $6815(3)$ & $8846(2)$ & $8363(1)$ & $32(1)$ \\
\hline$C(61)$ & 1614(3) & $6105(2)$ & $7782(1)$ & $30(1)$ \\
\hline $\mathrm{C}(47)$ & $4206(3)$ & 7432(2) & 7981(1) & $27(1)$ \\
\hline$C(25)$ & $7362(3)$ & 11122(2) & $3988(1)$ & $29(1)$ \\
\hline $\mathrm{C}(17)$ & $7470(3)$ & $9358(2)$ & $3633(1)$ & $28(1)$ \\
\hline$C(20)$ & $4566(3)$ & $8423(2)$ & $3118(1)$ & $27(1)$ \\
\hline $\mathrm{C}(50)$ & $3236(3)$ & 9031(2) & $8279(1)$ & $27(1)$ \\
\hline$C(43)$ & $7260(3)$ & $8973(2)$ & $5507(1)$ & $34(1)$ \\
\hline$C(44)$ & $7537(3)$ & $8420(2)$ & $5937(1)$ & $29(1)$ \\
\hline $\mathrm{C}(21)$ & $7874(3)$ & $7724(2)$ & $3413(1)$ & $30(1)$ \\
\hline$C(16)$ & $6867(3)$ & 8301(2) & $3368(1)$ & $27(1)$ \\
\hline$C(7)$ & $3065(3)$ & $6298(2)$ & $377(1)$ & $31(1)$ \\
\hline$C(27)$ & $7285(3)$ & $11283(2)$ & $4718(1)$ & $39(1)$ \\
\hline $\mathrm{C}(57)$ & $2695(3)$ & $9885(2)$ & $8447(1)$ & $30(1)$ \\
\hline $\mathrm{C}(38)$ & $7732(3)$ & $5831(2)$ & $5142(1)$ & $31(1)$ \\
\hline$C(12)$ & $4318(3)$ & $6495(2)$ & 178(1) & $36(1)$ \\
\hline$C(6)$ & 1379(3) & $3222(2)$ & $329(1)$ & $35(1)$ \\
\hline$C(46)$ & $4929(2)$ & 6491(2) & $6907(1)$ & $27(1)$ \\
\hline$C(36)$ & $8366(3)$ & 4378(2) & $4960(1)$ & $32(1)$ \\
\hline$C(29)$ & 2939(3) & $8006(2)$ & $2867(1)$ & $31(1)$ \\
\hline $\mathrm{C}(37)$ & $8102(3)$ & $5137(2)$ & $4739(1)$ & $33(1)$ \\
\hline
\end{tabular}




\begin{tabular}{|c|c|c|c|c|}
\hline$C(33)$ & $7598(2)$ & $5771(2)$ & $5784(1)$ & $26(1)$ \\
\hline $\mathrm{C}(41)$ & $5226(3)$ & $7513(2)$ & $4859(1)$ & $31(1)$ \\
\hline$C(42)$ & 6104(3) & $8521(2)$ & $4967(1)$ & $32(1)$ \\
\hline $\mathrm{C}(13)$ & 3988(3) & $5374(2)$ & 1337(1) & $34(1)$ \\
\hline $\mathrm{C}(39)$ & $6682(2)$ & $7401(2)$ & $5826(1)$ & $26(1)$ \\
\hline $\mathrm{C}(40)$ & $5506(3)$ & $6955(2)$ & $5285(1)$ & $29(1)$ \\
\hline $\mathrm{C}(5)$ & $921(3)$ & $2286(2)$ & $-140(1)$ & $40(1)$ \\
\hline $\mathrm{C}(51)$ & 2321(3) & $8009(2)$ & 8101(1) & $28(1)$ \\
\hline $\mathrm{C}(9)$ & 2739(3) & $7620(2)$ & $-81(1)$ & $40(1)$ \\
\hline $\mathrm{C}(28)$ & $6573(3)$ & $11678(2)$ & $3658(1)$ & $46(1)$ \\
\hline $\mathrm{C}(60)$ & $3760(3)$ & $10923(2)$ & $8456(1)$ & $37(1)$ \\
\hline $\mathrm{C}(34)$ & $7874(2)$ & 4997(2) & $6000(1)$ & $30(1)$ \\
\hline $\mathrm{C}(58)$ & $1343(3)$ & $9665(2)$ & $7928(1)$ & $43(1)$ \\
\hline $\mathrm{C}(1)$ & 1895(3) & $4130(2)$ & $156(1)$ & $29(1)$ \\
\hline $\mathrm{C}(35)$ & $8256(3)$ & $4313(2)$ & $5596(1)$ & $32(1)$ \\
\hline $\mathrm{C}(24)$ & $8228(3)$ & $7456(2)$ & 2733(1) & $34(1)$ \\
\hline $\mathrm{C}(23)$ & $7263(3)$ & $6748(2)$ & $3627(1)$ & $41(1)$ \\
\hline $\mathrm{C}(11)$ & $4766(3)$ & $7242(2)$ & $-148(1)$ & $42(1)$ \\
\hline $\mathrm{C}(45)$ & $5509(3)$ & $5863(2)$ & $6475(1)$ & $28(1)$ \\
\hline $\mathrm{C}(2)$ & 1924(3) & 4066(2) & $-508(1)$ & $40(1)$ \\
\hline $\mathrm{C}(10)$ & $3980(3)$ & 7804(2) & $-278(1)$ & $42(1)$ \\
\hline $\mathrm{C}(64)$ & $2060(3)$ & $5483(2)$ & $8213(1)$ & $41(1)$ \\
\hline$C(56)$ & $7271(3)$ & $9082(2)$ & $7744(1)$ & $47(1)$ \\
\hline$C(14)$ & $4648(3)$ & $6367(2)$ & $1872(1)$ & $31(1)$ \\
\hline $\mathrm{C}(63)$ & 204(3) & $6107(2)$ & $7915(1)$ & $41(1)$ \\
\hline $\mathrm{C}(22)$ & $9269(3)$ & $8385(2)$ & $3930(1)$ & $42(1)$ \\
\hline $\mathrm{C}(8)$ & $2287(3)$ & $6877(2)$ & $249(1)$ & $34(1)$ \\
\hline$C(62)$ & 1303(3) & $5554(2)$ & $7036(1)$ & $37(1)$ \\
\hline$C(26)$ & $8907(3)$ & 11599(2) & $3973(2)$ & $55(1)$ \\
\hline $\mathrm{C}(59)$ & $2387(3)$ & $9943(2)$ & $9138(1)$ & $44(1)$ \\
\hline $\mathrm{C}(4)$ & $950(3)$ & $2244(2)$ & $-795(1)$ & $42(1)$ \\
\hline$C(3)$ & $1448(3)$ & $3128(2)$ & $-974(1)$ & $47(1)$ \\
\hline $\mathrm{C}(30)$ & $2519(3)$ & 7808(3) & 2113(1) & $53(1)$ \\
\hline $\mathrm{C}(55)$ & $7436(3)$ & $8105(3)$ & $8569(2)$ & $65(1)$ \\
\hline $\mathrm{C}(32)$ & $2347(3)$ & $8770(2)$ & $3176(2)$ & $49(1)$ \\
\hline $\mathrm{C}(31)$ & 2194(3) & $7058(2)$ & $3081(2)$ & $63(1)$ \\
\hline
\end{tabular}


Table S8. Bond lengths $[\AA ̊]$ and angles $\left[^{\circ}\right]$ for cain09_a.

\begin{tabular}{|c|c|}
\hline $\mathrm{P}(3)-\mathrm{C}(33)$ & $1.833(2)$ \\
\hline $\mathrm{P}(3)-\mathrm{C}(39)$ & $1.836(2)$ \\
\hline $\mathrm{P}(3)-\mathrm{C}(45)$ & $1.867(2)$ \\
\hline $\mathrm{P}(4)-\mathrm{C}(47)$ & $1.859(2)$ \\
\hline $\mathrm{P}(4)-\mathrm{C}(46)$ & $1.663(2)$ \\
\hline $\mathrm{P}(2)-\mathrm{C}(15)$ & $1.857(2)$ \\
\hline $\mathrm{P}(2)-\mathrm{C}(14)$ & $1.659(3)$ \\
\hline $\mathrm{P}(1)-\mathrm{C}(7)$ & $1.838(2)$ \\
\hline $\mathrm{P}(1)-\mathrm{C}(13)$ & $1.868(3)$ \\
\hline $\mathrm{P}(1)-\mathrm{C}(1)$ & $1.834(2)$ \\
\hline $\mathrm{C}(19)-\mathrm{H}(19)$ & 0.9500 \\
\hline$C(19)-C(18)$ & $1.386(3)$ \\
\hline$C(19)-C(20)$ & $1.398(3)$ \\
\hline $\mathrm{C}(48)-\mathrm{C}(49)$ & $1.402(3)$ \\
\hline$C(48)-C(53)$ & $1.556(3)$ \\
\hline $\mathrm{C}(48)-\mathrm{C}(47)$ & $1.423(3)$ \\
\hline$C(18)-C(25)$ & $1.532(3)$ \\
\hline $\mathrm{C}(18)-\mathrm{C}(17)$ & $1.391(3)$ \\
\hline $\mathrm{C}(49)-\mathrm{H}(49)$ & 0.9500 \\
\hline$C(49)-C(50)$ & $1.380(3)$ \\
\hline$C(15)-C(20)$ & $1.425(3)$ \\
\hline$C(15)-C(16)$ & $1.421(3)$ \\
\hline$C(52)-C(61)$ & $1.555(3)$ \\
\hline$C(52)-C(47)$ & $1.427(3)$ \\
\hline$C(52)-C(51)$ & $1.389(3)$ \\
\hline$C(53)-C(56)$ & $1.524(3)$ \\
\hline$C(53)-C(55)$ & $1.534(4)$ \\
\hline$C(53)-C(54)$ & $1.535(4)$ \\
\hline$C(61)-C(64)$ & $1.536(3)$ \\
\hline$C(61)-C(63)$ & $1.543(4)$ \\
\hline$C(61)-C(62)$ & $1.542(3)$ \\
\hline$C(25)-C(27)$ & $1.531(3)$ \\
\hline$C(25)-C(28)$ & $1.526(3)$ \\
\hline$C(25)-C(26)$ & $1.530(4)$ \\
\hline
\end{tabular}




\begin{tabular}{|c|c|}
\hline $\mathrm{C}(17)-\mathrm{H}(17)$ & 0.9500 \\
\hline$C(17)-C(16)$ & $1.396(3)$ \\
\hline$C(20)-C(29)$ & $1.554(3)$ \\
\hline$C(50)-C(57)$ & $1.535(3)$ \\
\hline$C(50)-C(51)$ & $1.395(3)$ \\
\hline $\mathrm{C}(43)-\mathrm{H}(43)$ & 0.9500 \\
\hline $\mathrm{C}(43)-\mathrm{C}(44)$ & $1.390(3)$ \\
\hline $\mathrm{C}(43)-\mathrm{C}(42)$ & $1.382(4)$ \\
\hline $\mathrm{C}(44)-\mathrm{H}(44)$ & 0.9500 \\
\hline C(44)-C(39) & $1.389(3)$ \\
\hline$C(21)-C(16)$ & $1.558(3)$ \\
\hline$C(21)-C(24)$ & $1.545(3)$ \\
\hline$C(21)-C(23)$ & $1.535(3)$ \\
\hline$C(21)-C(22)$ & $1.535(4)$ \\
\hline$C(7)-C(12)$ & $1.390(4)$ \\
\hline $\mathrm{C}(7)-\mathrm{C}(8)$ & $1.391(3)$ \\
\hline $\mathrm{C}(27)-\mathrm{H}(27 \mathrm{~A})$ & 0.9800 \\
\hline $\mathrm{C}(27)-\mathrm{H}(27 \mathrm{~B})$ & 0.9800 \\
\hline $\mathrm{C}(27)-\mathrm{H}(27 \mathrm{C})$ & 0.9800 \\
\hline C(57)-C(60) & $1.530(3)$ \\
\hline C(57)-C(58) & $1.538(4)$ \\
\hline C(57)-C(59) & $1.535(3)$ \\
\hline $\mathrm{C}(38)-\mathrm{H}(38)$ & 0.9500 \\
\hline $\mathrm{C}(38)-\mathrm{C}(37)$ & $1.383(3)$ \\
\hline $\mathrm{C}(38)-\mathrm{C}(33)$ & $1.403(3)$ \\
\hline $\mathrm{C}(12)-\mathrm{H}(12)$ & 0.9500 \\
\hline $\mathrm{C}(12)-\mathrm{C}(11)$ & $1.391(3)$ \\
\hline $\mathrm{C}(6)-\mathrm{H}(6)$ & 0.9500 \\
\hline $\mathrm{C}(6)-\mathrm{C}(5)$ & $1.385(4)$ \\
\hline $\mathrm{C}(6)-\mathrm{C}(1)$ & $1.391(3)$ \\
\hline$C(46)-C(45)$ & $1.499(3)$ \\
\hline C(46)-H(46) & $0.95(2)$ \\
\hline $\mathrm{C}(36)-\mathrm{H}(36)$ & 0.9500 \\
\hline C(36)-C(37) & $1.376(3)$ \\
\hline$C(36)-C(35)$ & $1.386(3)$ \\
\hline$C(29)-C(30)$ & $1.523(4)$ \\
\hline
\end{tabular}




\begin{tabular}{|c|c|}
\hline$C(29)-C(32)$ & $1.532(4)$ \\
\hline$C(29)-C(31)$ & $1.528(3)$ \\
\hline $\mathrm{C}(37)-\mathrm{H}(37)$ & 0.9500 \\
\hline$C(33)-C(34)$ & $1.396(3)$ \\
\hline $\mathrm{C}(41)-\mathrm{H}(41)$ & 0.9500 \\
\hline $\mathrm{C}(41)-\mathrm{C}(42)$ & $1.384(3)$ \\
\hline $\mathrm{C}(41)-\mathrm{C}(40)$ & $1.390(3)$ \\
\hline $\mathrm{C}(42)-\mathrm{H}(42)$ & 0.9500 \\
\hline $\mathrm{C}(13)-\mathrm{H}(13 \mathrm{~A})$ & 0.9900 \\
\hline $\mathrm{C}(13)-\mathrm{H}(13 \mathrm{~B})$ & 0.9900 \\
\hline$C(13)-C(14)$ & $1.500(3)$ \\
\hline$C(39)-C(40)$ & $1.397(3)$ \\
\hline $\mathrm{C}(40)-\mathrm{H}(40)$ & 0.9500 \\
\hline $\mathrm{C}(5)-\mathrm{H}(5)$ & 0.9500 \\
\hline $\mathrm{C}(5)-\mathrm{C}(4)$ & $1.380(4)$ \\
\hline $\mathrm{C}(51)-\mathrm{H}(51)$ & 0.9500 \\
\hline $\mathrm{C}(9)-\mathrm{H}(9)$ & 0.9500 \\
\hline $\mathrm{C}(9)-\mathrm{C}(10)$ & $1.378(4)$ \\
\hline $\mathrm{C}(9)-\mathrm{C}(8)$ & $1.391(3)$ \\
\hline $\mathrm{C}(28)-\mathrm{H}(28 \mathrm{~A})$ & 0.9800 \\
\hline $\mathrm{C}(28)-\mathrm{H}(28 \mathrm{~B})$ & 0.9800 \\
\hline $\mathrm{C}(28)-\mathrm{H}(28 \mathrm{C})$ & 0.9800 \\
\hline $\mathrm{C}(60)-\mathrm{H}(60 \mathrm{~A})$ & 0.9800 \\
\hline $\mathrm{C}(60)-\mathrm{H}(60 \mathrm{~B})$ & 0.9800 \\
\hline $\mathrm{C}(60)-\mathrm{H}(60 \mathrm{C})$ & 0.9800 \\
\hline C(34)-H(34) & 0.9500 \\
\hline$C(34)-C(35)$ & $1.383(3)$ \\
\hline $\mathrm{C}(58)-\mathrm{H}(58 \mathrm{~A})$ & 0.9800 \\
\hline C(58)-H(58B) & 0.9800 \\
\hline $\mathrm{C}(58)-\mathrm{H}(58 \mathrm{C})$ & 0.9800 \\
\hline $\mathrm{C}(1)-\mathrm{C}(2)$ & $1.393(3)$ \\
\hline C(35)-H(35) & 0.9500 \\
\hline $\mathrm{C}(24)-\mathrm{H}(24 \mathrm{~A})$ & 0.9800 \\
\hline $\mathrm{C}(24)-\mathrm{H}(24 \mathrm{~B})$ & 0.9800 \\
\hline $\mathrm{C}(24)-\mathrm{H}(24 \mathrm{C})$ & 0.9800 \\
\hline $\mathrm{C}(23)-\mathrm{H}(23 \mathrm{~A})$ & 0.9800 \\
\hline
\end{tabular}




\begin{tabular}{|c|c|}
\hline $\mathrm{C}(23)-\mathrm{H}(23 \mathrm{~B})$ & 0.9800 \\
\hline $\mathrm{C}(23)-\mathrm{H}(23 \mathrm{C})$ & 0.9800 \\
\hline $\mathrm{C}(11)-\mathrm{H}(11)$ & 0.9500 \\
\hline$C(11)-C(10)$ & $1.379(4)$ \\
\hline $\mathrm{C}(45)-\mathrm{H}(45 \mathrm{~A})$ & 0.9900 \\
\hline $\mathrm{C}(45)-\mathrm{H}(45 \mathrm{~B})$ & 0.9900 \\
\hline $\mathrm{C}(2)-\mathrm{H}(2)$ & 0.9500 \\
\hline $\mathrm{C}(2)-\mathrm{C}(3)$ & $1.382(4)$ \\
\hline $\mathrm{C}(10)-\mathrm{H}(10)$ & 0.9500 \\
\hline $\mathrm{C}(64)-\mathrm{H}(64 \mathrm{~A})$ & 0.9800 \\
\hline C(64)-H(64B) & 0.9800 \\
\hline $\mathrm{C}(64)-\mathrm{H}(64 \mathrm{C})$ & 0.9800 \\
\hline $\mathrm{C}(56)-\mathrm{H}(56 \mathrm{~A})$ & 0.9800 \\
\hline $\mathrm{C}(56)-\mathrm{H}(56 \mathrm{~B})$ & 0.9800 \\
\hline $\mathrm{C}(56)-\mathrm{H}(56 \mathrm{C})$ & 0.9800 \\
\hline $\mathrm{C}(14)-\mathrm{H}(14)$ & $0.94(2)$ \\
\hline $\mathrm{C}(63)-\mathrm{H}(63 \mathrm{~A})$ & 0.9800 \\
\hline C(63)-H(63B) & 0.9800 \\
\hline $\mathrm{C}(63)-\mathrm{H}(63 \mathrm{C})$ & 0.9800 \\
\hline $\mathrm{C}(22)-\mathrm{H}(22 \mathrm{~A})$ & 0.9800 \\
\hline $\mathrm{C}(22)-\mathrm{H}(22 \mathrm{~B})$ & 0.9800 \\
\hline $\mathrm{C}(22)-\mathrm{H}(22 \mathrm{C})$ & 0.9800 \\
\hline $\mathrm{C}(8)-\mathrm{H}(8)$ & 0.9500 \\
\hline $\mathrm{C}(62)-\mathrm{H}(62 \mathrm{~A})$ & 0.9800 \\
\hline $\mathrm{C}(62)-\mathrm{H}(62 \mathrm{~B})$ & 0.9800 \\
\hline $\mathrm{C}(62)-\mathrm{H}(62 \mathrm{C})$ & 0.9800 \\
\hline $\mathrm{C}(26)-\mathrm{H}(26 \mathrm{~A})$ & 0.9800 \\
\hline $\mathrm{C}(26)-\mathrm{H}(26 \mathrm{~B})$ & 0.9800 \\
\hline $\mathrm{C}(26)-\mathrm{H}(26 \mathrm{C})$ & 0.9800 \\
\hline C(59)-H(59A) & 0.9800 \\
\hline $\mathrm{C}(59)-\mathrm{H}(59 \mathrm{~B})$ & 0.9800 \\
\hline C(59)-H(59C) & 0.9800 \\
\hline $\mathrm{C}(4)-\mathrm{H}(4)$ & 0.9500 \\
\hline$C(4)-C(3)$ & $1.365(4)$ \\
\hline $\mathrm{C}(3)-\mathrm{H}(3)$ & 0.9500 \\
\hline $\mathrm{C}(30)-\mathrm{H}(30 \mathrm{~A})$ & 0.9800 \\
\hline
\end{tabular}




\begin{tabular}{|c|c|}
\hline $\mathrm{C}(30)-\mathrm{H}(30 \mathrm{~B})$ & 0.9800 \\
\hline $\mathrm{C}(30)-\mathrm{H}(30 \mathrm{C})$ & 0.9800 \\
\hline $\mathrm{C}(55)-\mathrm{H}(55 \mathrm{~A})$ & 0.9800 \\
\hline $\mathrm{C}(55)-\mathrm{H}(55 \mathrm{~B})$ & 0.9800 \\
\hline $\mathrm{C}(55)-\mathrm{H}(55 \mathrm{C})$ & 0.9800 \\
\hline $\mathrm{C}(32)-\mathrm{H}(32 \mathrm{~A})$ & 0.9800 \\
\hline $\mathrm{C}(32)-\mathrm{H}(32 \mathrm{~B})$ & 0.9800 \\
\hline $\mathrm{C}(32)-\mathrm{H}(32 \mathrm{C})$ & 0.9800 \\
\hline $\mathrm{C}(31)-\mathrm{H}(31 \mathrm{~A})$ & 0.9800 \\
\hline $\mathrm{C}(31)-\mathrm{H}(31 \mathrm{~B})$ & 0.9800 \\
\hline $\mathrm{C}(31)-\mathrm{H}(31 \mathrm{C})$ & 0.9800 \\
\hline $\mathrm{C}(54)-\mathrm{H}(54 \mathrm{~A})$ & 0.9800 \\
\hline C(54)-H(54B) & 0.9800 \\
\hline $\mathrm{C}(54)-\mathrm{H}(54 \mathrm{C})$ & 0.9800 \\
\hline C(33)-P(3)-C(39) & $101.28(10)$ \\
\hline C(33)-P(3)-C(45) & $99.93(10)$ \\
\hline C(39)-P(3)-C(45) & $101.91(11)$ \\
\hline C(46)-P(4)-C(47) & $102.59(11)$ \\
\hline $\mathrm{C}(14)-\mathrm{P}(2)-\mathrm{C}(15)$ & $103.56(11)$ \\
\hline $\mathrm{C}(7)-\mathrm{P}(1)-\mathrm{C}(13)$ & $101.56(12)$ \\
\hline $\mathrm{C}(1)-\mathrm{P}(1)-\mathrm{C}(7)$ & $102.65(11)$ \\
\hline $\mathrm{C}(1)-\mathrm{P}(1)-\mathrm{C}(13)$ & $99.82(11)$ \\
\hline $\mathrm{C}(18)-\mathrm{C}(19)-\mathrm{H}(19)$ & 118.0 \\
\hline $\mathrm{C}(18)-\mathrm{C}(19)-\mathrm{C}(20)$ & $124.0(2)$ \\
\hline $\mathrm{C}(20)-\mathrm{C}(19)-\mathrm{H}(19)$ & 118.0 \\
\hline C(49)-C(48)-C(53) & $115.6(2)$ \\
\hline $\mathrm{C}(49)-\mathrm{C}(48)-\mathrm{C}(47)$ & $117.7(2)$ \\
\hline $\mathrm{C}(47)-\mathrm{C}(48)-\mathrm{C}(53)$ & $126.7(2)$ \\
\hline $\mathrm{C}(19)-\mathrm{C}(18)-\mathrm{C}(25)$ & $120.5(2)$ \\
\hline C(19)-C(18)-C(17) & $116.4(2)$ \\
\hline $\mathrm{C}(17)-\mathrm{C}(18)-\mathrm{C}(25)$ & $122.8(2)$ \\
\hline $\mathrm{C}(48)-\mathrm{C}(49)-\mathrm{H}(49)$ & 118.0 \\
\hline $\mathrm{C}(50)-\mathrm{C}(49)-\mathrm{C}(48)$ & $123.9(2)$ \\
\hline C(50)-C(49)-H(49) & 118.0 \\
\hline $\mathrm{C}(20)-\mathrm{C}(15)-\mathrm{P}(2)$ & $119.89(18)$ \\
\hline
\end{tabular}




\begin{tabular}{|c|c|}
\hline$C(16)-C(15)-P(2)$ & $120.79(17)$ \\
\hline $\mathrm{C}(16)-\mathrm{C}(15)-\mathrm{C}(20)$ & 119.3(2) \\
\hline$C(47)-C(52)-C(61)$ & $124.2(2)$ \\
\hline $\mathrm{C}(51)-\mathrm{C}(52)-\mathrm{C}(61)$ & $117.5(2)$ \\
\hline $\mathrm{C}(51)-\mathrm{C}(52)-\mathrm{C}(47)$ & $118.4(2)$ \\
\hline $\mathrm{C}(56)-\mathrm{C}(53)-\mathrm{C}(48)$ & 109.2(2) \\
\hline$C(56)-C(53)-C(55)$ & $108.7(2)$ \\
\hline$C(56)-C(53)-C(54)$ & $107.9(2)$ \\
\hline $\mathrm{C}(55)-\mathrm{C}(53)-\mathrm{C}(48)$ & $115.0(2)$ \\
\hline $\mathrm{C}(55)-\mathrm{C}(53)-\mathrm{C}(54)$ & $105.7(2)$ \\
\hline$C(54)-C(53)-C(48)$ & $110.1(2)$ \\
\hline $\mathrm{C}(64)-\mathrm{C}(61)-\mathrm{C}(52)$ & 111.3(2) \\
\hline$C(64)-C(61)-C(63)$ & $105.9(2)$ \\
\hline$C(64)-C(61)-C(62)$ & $110.2(2)$ \\
\hline $\mathrm{C}(63)-\mathrm{C}(61)-\mathrm{C}(52)$ & $111.9(2)$ \\
\hline $\mathrm{C}(62)-\mathrm{C}(61)-\mathrm{C}(52)$ & $110.90(19)$ \\
\hline $\mathrm{C}(62)-\mathrm{C}(61)-\mathrm{C}(63)$ & $106.4(2)$ \\
\hline $\mathrm{C}(48)-\mathrm{C}(47)-\mathrm{P}(4)$ & $120.02(18)$ \\
\hline C(48)-C(47)-C(52) & 119.4(2) \\
\hline $\mathrm{C}(52)-\mathrm{C}(47)-\mathrm{P}(4)$ & $120.56(18)$ \\
\hline $\mathrm{C}(27)-\mathrm{C}(25)-\mathrm{C}(18)$ & $107.63(19)$ \\
\hline $\mathrm{C}(28)-\mathrm{C}(25)-\mathrm{C}(18)$ & $111.4(2)$ \\
\hline $\mathrm{C}(28)-\mathrm{C}(25)-\mathrm{C}(27)$ & 109.1(2) \\
\hline $\mathrm{C}(28)-\mathrm{C}(25)-\mathrm{C}(26)$ & $108.1(2)$ \\
\hline $\mathrm{C}(26)-\mathrm{C}(25)-\mathrm{C}(18)$ & $112.4(2)$ \\
\hline $\mathrm{C}(26)-\mathrm{C}(25)-\mathrm{C}(27)$ & $108.1(2)$ \\
\hline $\mathrm{C}(18)-\mathrm{C}(17)-\mathrm{H}(17)$ & 118.2 \\
\hline $\mathrm{C}(18)-\mathrm{C}(17)-\mathrm{C}(16)$ & $123.5(2)$ \\
\hline $\mathrm{C}(16)-\mathrm{C}(17)-\mathrm{H}(17)$ & 118.2 \\
\hline C(19)-C(20)-C(15) & $118.0(2)$ \\
\hline C(19)-C(20)-C(29) & $116.5(2)$ \\
\hline$C(15)-C(20)-C(29)$ & $125.4(2)$ \\
\hline C(49)-C(50)-C(57) & $122.4(2)$ \\
\hline $\mathrm{C}(49)-\mathrm{C}(50)-\mathrm{C}(51)$ & $116.6(2)$ \\
\hline $\mathrm{C}(51)-\mathrm{C}(50)-\mathrm{C}(57)$ & $120.8(2)$ \\
\hline $\mathrm{C}(44)-\mathrm{C}(43)-\mathrm{H}(43)$ & 119.8 \\
\hline
\end{tabular}




\begin{tabular}{|c|c|}
\hline $\mathrm{C}(42)-\mathrm{C}(43)-\mathrm{H}(43)$ & 119.8 \\
\hline$C(42)-C(43)-C(44)$ & $120.3(2)$ \\
\hline $\mathrm{C}(43)-\mathrm{C}(44)-\mathrm{H}(44)$ & 119.5 \\
\hline$C(39)-C(44)-C(43)$ & $120.9(2)$ \\
\hline $\mathrm{C}(39)-\mathrm{C}(44)-\mathrm{H}(44)$ & 119.5 \\
\hline$C(24)-C(21)-C(16)$ & $109.88(19)$ \\
\hline$C(23)-C(21)-C(16)$ & $113.0(2)$ \\
\hline$C(23)-C(21)-C(24)$ & $109.4(2)$ \\
\hline$C(23)-C(21)-C(22)$ & $105.9(2)$ \\
\hline$C(22)-C(21)-C(16)$ & $111.3(2)$ \\
\hline $\mathrm{C}(22)-\mathrm{C}(21)-\mathrm{C}(24)$ & $107.2(2)$ \\
\hline$C(15)-C(16)-C(21)$ & $124.5(2)$ \\
\hline$C(17)-C(16)-C(15)$ & $118.5(2)$ \\
\hline $\mathrm{C}(17)-\mathrm{C}(16)-\mathrm{C}(21)$ & $116.9(2)$ \\
\hline $\mathrm{C}(12)-\mathrm{C}(7)-\mathrm{P}(1)$ & $124.7(2)$ \\
\hline $\mathrm{C}(12)-\mathrm{C}(7)-\mathrm{C}(8)$ & $118.4(2)$ \\
\hline $\mathrm{C}(8)-\mathrm{C}(7)-\mathrm{P}(1)$ & 116.97(19) \\
\hline $\mathrm{C}(25)-\mathrm{C}(27)-\mathrm{H}(27 \mathrm{~A})$ & 109.5 \\
\hline $\mathrm{C}(25)-\mathrm{C}(27)-\mathrm{H}(27 \mathrm{~B})$ & 109.5 \\
\hline $\mathrm{C}(25)-\mathrm{C}(27)-\mathrm{H}(27 \mathrm{C})$ & 109.5 \\
\hline $\mathrm{H}(27 \mathrm{~A})-\mathrm{C}(27)-\mathrm{H}(27 \mathrm{~B})$ & 109.5 \\
\hline $\mathrm{H}(27 \mathrm{~A})-\mathrm{C}(27)-\mathrm{H}(27 \mathrm{C})$ & 109.5 \\
\hline $\mathrm{H}(27 \mathrm{~B})-\mathrm{C}(27)-\mathrm{H}(27 \mathrm{C})$ & 109.5 \\
\hline $\mathrm{C}(50)-\mathrm{C}(57)-\mathrm{C}(58)$ & $110.7(2)$ \\
\hline $\mathrm{C}(50)-\mathrm{C}(57)-\mathrm{C}(59)$ & $108.07(19)$ \\
\hline$C(60)-C(57)-C(50)$ & $112.4(2)$ \\
\hline $\mathrm{C}(60)-\mathrm{C}(57)-\mathrm{C}(58)$ & $107.0(2)$ \\
\hline$C(60)-C(57)-C(59)$ & 109.1(2) \\
\hline $\mathrm{C}(59)-\mathrm{C}(57)-\mathrm{C}(58)$ & $109.6(2)$ \\
\hline $\mathrm{C}(37)-\mathrm{C}(38)-\mathrm{H}(38)$ & 119.6 \\
\hline $\mathrm{C}(37)-\mathrm{C}(38)-\mathrm{C}(33)$ & $120.9(2)$ \\
\hline $\mathrm{C}(33)-\mathrm{C}(38)-\mathrm{H}(38)$ & 119.6 \\
\hline $\mathrm{C}(7)-\mathrm{C}(12)-\mathrm{H}(12)$ & 119.7 \\
\hline $\mathrm{C}(7)-\mathrm{C}(12)-\mathrm{C}(11)$ & $120.5(3)$ \\
\hline $\mathrm{C}(11)-\mathrm{C}(12)-\mathrm{H}(12)$ & 119.7 \\
\hline $\mathrm{C}(5)-\mathrm{C}(6)-\mathrm{H}(6)$ & 119.3 \\
\hline
\end{tabular}




\begin{tabular}{|c|c|}
\hline$C(5)-C(6)-C(1)$ & $121.3(2)$ \\
\hline$C(1)-C(6)-H(6)$ & 119.3 \\
\hline $\mathrm{P}(4)-\mathrm{C}(46)-\mathrm{H}(46)$ & $120.1(13)$ \\
\hline $\mathrm{C}(45)-\mathrm{C}(46)-\mathrm{P}(4)$ & $122.34(18)$ \\
\hline $\mathrm{C}(45)-\mathrm{C}(46)-\mathrm{H}(46)$ & $117.5(13)$ \\
\hline $\mathrm{C}(37)-\mathrm{C}(36)-\mathrm{H}(36)$ & 120.5 \\
\hline $\mathrm{C}(37)-\mathrm{C}(36)-\mathrm{C}(35)$ & 119.1(2) \\
\hline $\mathrm{C}(35)-\mathrm{C}(36)-\mathrm{H}(36)$ & 120.5 \\
\hline$C(30)-C(29)-C(20)$ & $110.3(2)$ \\
\hline$C(30)-C(29)-C(32)$ & $107.3(2)$ \\
\hline$C(30)-C(29)-C(31)$ & $111.4(2)$ \\
\hline$C(32)-C(29)-C(20)$ & $111.5(2)$ \\
\hline$C(31)-C(29)-C(20)$ & $112.6(2)$ \\
\hline$C(31)-C(29)-C(32)$ & $103.6(2)$ \\
\hline $\mathrm{C}(38)-\mathrm{C}(37)-\mathrm{H}(37)$ & 119.5 \\
\hline $\mathrm{C}(36)-\mathrm{C}(37)-\mathrm{C}(38)$ & $120.9(2)$ \\
\hline $\mathrm{C}(36)-\mathrm{C}(37)-\mathrm{H}(37)$ & 119.5 \\
\hline C(38)-C(33)-P(3) & $123.97(18)$ \\
\hline $\mathrm{C}(34)-\mathrm{C}(33)-\mathrm{P}(3)$ & $118.43(18)$ \\
\hline $\mathrm{C}(34)-\mathrm{C}(33)-\mathrm{C}(38)$ & $117.4(2)$ \\
\hline $\mathrm{C}(42)-\mathrm{C}(41)-\mathrm{H}(41)$ & 119.8 \\
\hline $\mathrm{C}(42)-\mathrm{C}(41)-\mathrm{C}(40)$ & $120.5(2)$ \\
\hline $\mathrm{C}(40)-\mathrm{C}(41)-\mathrm{H}(41)$ & 119.8 \\
\hline $\mathrm{C}(43)-\mathrm{C}(42)-\mathrm{C}(41)$ & $119.4(2)$ \\
\hline $\mathrm{C}(43)-\mathrm{C}(42)-\mathrm{H}(42)$ & 120.3 \\
\hline $\mathrm{C}(41)-\mathrm{C}(42)-\mathrm{H}(42)$ & 120.3 \\
\hline $\mathrm{P}(1)-\mathrm{C}(13)-\mathrm{H}(13 \mathrm{~A})$ & 109.8 \\
\hline $\mathrm{P}(1)-\mathrm{C}(13)-\mathrm{H}(13 \mathrm{~B})$ & 109.8 \\
\hline $\mathrm{H}(13 \mathrm{~A})-\mathrm{C}(13)-\mathrm{H}(13 \mathrm{~B})$ & 108.3 \\
\hline $\mathrm{C}(14)-\mathrm{C}(13)-\mathrm{P}(1)$ & $109.34(17)$ \\
\hline $\mathrm{C}(14)-\mathrm{C}(13)-\mathrm{H}(13 \mathrm{~A})$ & 109.8 \\
\hline $\mathrm{C}(14)-\mathrm{C}(13)-\mathrm{H}(13 \mathrm{~B})$ & 109.8 \\
\hline $\mathrm{C}(44)-\mathrm{C}(39)-\mathrm{P}(3)$ & $117.62(18)$ \\
\hline $\mathrm{C}(44)-\mathrm{C}(39)-\mathrm{C}(40)$ & $118.3(2)$ \\
\hline $\mathrm{C}(40)-\mathrm{C}(39)-\mathrm{P}(3)$ & $123.94(18)$ \\
\hline $\mathrm{C}(41)-\mathrm{C}(40)-\mathrm{C}(39)$ & $120.5(2)$ \\
\hline
\end{tabular}




\begin{tabular}{|c|c|}
\hline $\mathrm{C}(41)-\mathrm{C}(40)-\mathrm{H}(40)$ & 119.7 \\
\hline $\mathrm{C}(39)-\mathrm{C}(40)-\mathrm{H}(40)$ & 119.7 \\
\hline $\mathrm{C}(6)-\mathrm{C}(5)-\mathrm{H}(5)$ & 120.1 \\
\hline $\mathrm{C}(4)-\mathrm{C}(5)-\mathrm{C}(6)$ & $119.9(2)$ \\
\hline $\mathrm{C}(4)-\mathrm{C}(5)-\mathrm{H}(5)$ & 120.1 \\
\hline $\mathrm{C}(52)-\mathrm{C}(51)-\mathrm{C}(50)$ & $123.4(2)$ \\
\hline $\mathrm{C}(52)-\mathrm{C}(51)-\mathrm{H}(51)$ & 118.3 \\
\hline $\mathrm{C}(50)-\mathrm{C}(51)-\mathrm{H}(51)$ & 118.3 \\
\hline $\mathrm{C}(10)-\mathrm{C}(9)-\mathrm{H}(9)$ & 119.9 \\
\hline $\mathrm{C}(10)-\mathrm{C}(9)-\mathrm{C}(8)$ & $120.3(3)$ \\
\hline $\mathrm{C}(8)-\mathrm{C}(9)-\mathrm{H}(9)$ & 119.9 \\
\hline $\mathrm{C}(25)-\mathrm{C}(28)-\mathrm{H}(28 \mathrm{~A})$ & 109.5 \\
\hline $\mathrm{C}(25)-\mathrm{C}(28)-\mathrm{H}(28 \mathrm{~B})$ & 109.5 \\
\hline $\mathrm{C}(25)-\mathrm{C}(28)-\mathrm{H}(28 \mathrm{C})$ & 109.5 \\
\hline $\mathrm{H}(28 \mathrm{~A})-\mathrm{C}(28)-\mathrm{H}(28 \mathrm{~B})$ & 109.5 \\
\hline $\mathrm{H}(28 \mathrm{~A})-\mathrm{C}(28)-\mathrm{H}(28 \mathrm{C})$ & 109.5 \\
\hline $\mathrm{H}(28 \mathrm{~B})-\mathrm{C}(28)-\mathrm{H}(28 \mathrm{C})$ & 109.5 \\
\hline $\mathrm{C}(57)-\mathrm{C}(60)-\mathrm{H}(60 \mathrm{~A})$ & 109.5 \\
\hline $\mathrm{C}(57)-\mathrm{C}(60)-\mathrm{H}(60 \mathrm{~B})$ & 109.5 \\
\hline $\mathrm{C}(57)-\mathrm{C}(60)-\mathrm{H}(60 \mathrm{C})$ & 109.5 \\
\hline $\mathrm{H}(60 \mathrm{~A})-\mathrm{C}(60)-\mathrm{H}(60 \mathrm{~B})$ & 109.5 \\
\hline $\mathrm{H}(60 \mathrm{~A})-\mathrm{C}(60)-\mathrm{H}(60 \mathrm{C})$ & 109.5 \\
\hline $\mathrm{H}(60 \mathrm{~B})-\mathrm{C}(60)-\mathrm{H}(60 \mathrm{C})$ & 109.5 \\
\hline $\mathrm{C}(33)-\mathrm{C}(34)-\mathrm{H}(34)$ & 119.4 \\
\hline $\mathrm{C}(35)-\mathrm{C}(34)-\mathrm{C}(33)$ & $121.3(2)$ \\
\hline $\mathrm{C}(35)-\mathrm{C}(34)-\mathrm{H}(34)$ & 119.4 \\
\hline $\mathrm{C}(57)-\mathrm{C}(58)-\mathrm{H}(58 \mathrm{~A})$ & 109.5 \\
\hline $\mathrm{C}(57)-\mathrm{C}(58)-\mathrm{H}(58 \mathrm{~B})$ & 109.5 \\
\hline $\mathrm{C}(57)-\mathrm{C}(58)-\mathrm{H}(58 \mathrm{C})$ & 109.5 \\
\hline $\mathrm{H}(58 \mathrm{~A})-\mathrm{C}(58)-\mathrm{H}(58 \mathrm{~B})$ & 109.5 \\
\hline $\mathrm{H}(58 \mathrm{~A})-\mathrm{C}(58)-\mathrm{H}(58 \mathrm{C})$ & 109.5 \\
\hline $\mathrm{H}(58 \mathrm{~B})-\mathrm{C}(58)-\mathrm{H}(58 \mathrm{C})$ & 109.5 \\
\hline $\mathrm{C}(6)-\mathrm{C}(1)-\mathrm{P}(1)$ & $117.62(18)$ \\
\hline$C(6)-C(1)-C(2)$ & $117.6(2)$ \\
\hline $\mathrm{C}(2)-\mathrm{C}(1)-\mathrm{P}(1)$ & $124.56(19)$ \\
\hline $\mathrm{C}(36)-\mathrm{C}(35)-\mathrm{H}(35)$ & 119.8 \\
\hline
\end{tabular}




\begin{tabular}{|c|c|}
\hline $\mathrm{C}(34)-\mathrm{C}(35)-\mathrm{C}(36)$ & $120.4(2)$ \\
\hline $\mathrm{C}(34)-\mathrm{C}(35)-\mathrm{H}(35)$ & 119.8 \\
\hline $\mathrm{C}(21)-\mathrm{C}(24)-\mathrm{H}(24 \mathrm{~A})$ & 109.5 \\
\hline $\mathrm{C}(21)-\mathrm{C}(24)-\mathrm{H}(24 \mathrm{~B})$ & 109.5 \\
\hline $\mathrm{C}(21)-\mathrm{C}(24)-\mathrm{H}(24 \mathrm{C})$ & 109.5 \\
\hline $\mathrm{H}(24 \mathrm{~A})-\mathrm{C}(24)-\mathrm{H}(24 \mathrm{~B})$ & 109.5 \\
\hline $\mathrm{H}(24 \mathrm{~A})-\mathrm{C}(24)-\mathrm{H}(24 \mathrm{C})$ & 109.5 \\
\hline $\mathrm{H}(24 \mathrm{~B})-\mathrm{C}(24)-\mathrm{H}(24 \mathrm{C})$ & 109.5 \\
\hline $\mathrm{C}(21)-\mathrm{C}(23)-\mathrm{H}(23 \mathrm{~A})$ & 109.5 \\
\hline $\mathrm{C}(21)-\mathrm{C}(23)-\mathrm{H}(23 \mathrm{~B})$ & 109.5 \\
\hline $\mathrm{C}(21)-\mathrm{C}(23)-\mathrm{H}(23 \mathrm{C})$ & 109.5 \\
\hline $\mathrm{H}(23 \mathrm{~A})-\mathrm{C}(23)-\mathrm{H}(23 \mathrm{~B})$ & 109.5 \\
\hline $\mathrm{H}(23 \mathrm{~A})-\mathrm{C}(23)-\mathrm{H}(23 \mathrm{C})$ & 109.5 \\
\hline $\mathrm{H}(23 \mathrm{~B})-\mathrm{C}(23)-\mathrm{H}(23 \mathrm{C})$ & 109.5 \\
\hline $\mathrm{C}(12)-\mathrm{C}(11)-\mathrm{H}(11)$ & 119.7 \\
\hline $\mathrm{C}(10)-\mathrm{C}(11)-\mathrm{C}(12)$ & $120.6(3)$ \\
\hline $\mathrm{C}(10)-\mathrm{C}(11)-\mathrm{H}(11)$ & 119.7 \\
\hline $\mathrm{P}(3)-\mathrm{C}(45)-\mathrm{H}(45 \mathrm{~A})$ & 109.7 \\
\hline $\mathrm{P}(3)-\mathrm{C}(45)-\mathrm{H}(45 \mathrm{~B})$ & 109.7 \\
\hline $\mathrm{C}(46)-\mathrm{C}(45)-\mathrm{P}(3)$ & $109.68(16)$ \\
\hline $\mathrm{C}(46)-\mathrm{C}(45)-\mathrm{H}(45 \mathrm{~A})$ & 109.7 \\
\hline $\mathrm{C}(46)-\mathrm{C}(45)-\mathrm{H}(45 \mathrm{~B})$ & 109.7 \\
\hline $\mathrm{H}(45 \mathrm{~A})-\mathrm{C}(45)-\mathrm{H}(45 \mathrm{~B})$ & 108.2 \\
\hline $\mathrm{C}(1)-\mathrm{C}(2)-\mathrm{H}(2)$ & 119.7 \\
\hline $\mathrm{C}(3)-\mathrm{C}(2)-\mathrm{C}(1)$ & $120.6(2)$ \\
\hline $\mathrm{C}(3)-\mathrm{C}(2)-\mathrm{H}(2)$ & 119.7 \\
\hline $\mathrm{C}(9)-\mathrm{C}(10)-\mathrm{C}(11)$ & $119.5(2)$ \\
\hline $\mathrm{C}(9)-\mathrm{C}(10)-\mathrm{H}(10)$ & 120.2 \\
\hline $\mathrm{C}(11)-\mathrm{C}(10)-\mathrm{H}(10)$ & 120.2 \\
\hline $\mathrm{C}(61)-\mathrm{C}(64)-\mathrm{H}(64 \mathrm{~A})$ & 109.5 \\
\hline $\mathrm{C}(61)-\mathrm{C}(64)-\mathrm{H}(64 \mathrm{~B})$ & 109.5 \\
\hline $\mathrm{C}(61)-\mathrm{C}(64)-\mathrm{H}(64 \mathrm{C})$ & 109.5 \\
\hline $\mathrm{H}(64 \mathrm{~A})-\mathrm{C}(64)-\mathrm{H}(64 \mathrm{~B})$ & 109.5 \\
\hline $\mathrm{H}(64 \mathrm{~A})-\mathrm{C}(64)-\mathrm{H}(64 \mathrm{C})$ & 109.5 \\
\hline $\mathrm{H}(64 \mathrm{~B})-\mathrm{C}(64)-\mathrm{H}(64 \mathrm{C})$ & 109.5 \\
\hline $\mathrm{C}(53)-\mathrm{C}(56)-\mathrm{H}(56 \mathrm{~A})$ & 109.5 \\
\hline
\end{tabular}




\begin{tabular}{|c|c|}
\hline $\mathrm{C}(53)-\mathrm{C}(56)-\mathrm{H}(56 \mathrm{~B})$ & 109.5 \\
\hline $\mathrm{C}(53)-\mathrm{C}(56)-\mathrm{H}(56 \mathrm{C})$ & 109.5 \\
\hline $\mathrm{H}(56 \mathrm{~A})-\mathrm{C}(56)-\mathrm{H}(56 \mathrm{~B})$ & 109.5 \\
\hline $\mathrm{H}(56 \mathrm{~A})-\mathrm{C}(56)-\mathrm{H}(56 \mathrm{C})$ & 109.5 \\
\hline $\mathrm{H}(56 \mathrm{~B})-\mathrm{C}(56)-\mathrm{H}(56 \mathrm{C})$ & 109.5 \\
\hline $\mathrm{P}(2)-\mathrm{C}(14)-\mathrm{H}(14)$ & $122.3(14)$ \\
\hline $\mathrm{C}(13)-\mathrm{C}(14)-\mathrm{P}(2)$ & $121.67(19)$ \\
\hline $\mathrm{C}(13)-\mathrm{C}(14)-\mathrm{H}(14)$ & $115.9(14)$ \\
\hline $\mathrm{C}(61)-\mathrm{C}(63)-\mathrm{H}(63 \mathrm{~A})$ & 109.5 \\
\hline $\mathrm{C}(61)-\mathrm{C}(63)-\mathrm{H}(63 \mathrm{~B})$ & 109.5 \\
\hline $\mathrm{C}(61)-\mathrm{C}(63)-\mathrm{H}(63 \mathrm{C})$ & 109.5 \\
\hline $\mathrm{H}(63 \mathrm{~A})-\mathrm{C}(63)-\mathrm{H}(63 \mathrm{~B})$ & 109.5 \\
\hline $\mathrm{H}(63 \mathrm{~A})-\mathrm{C}(63)-\mathrm{H}(63 \mathrm{C})$ & 109.5 \\
\hline$H(63 B)-C(63)-H(63 C)$ & 109.5 \\
\hline $\mathrm{C}(21)-\mathrm{C}(22)-\mathrm{H}(22 \mathrm{~A})$ & 109.5 \\
\hline $\mathrm{C}(21)-\mathrm{C}(22)-\mathrm{H}(22 \mathrm{~B})$ & 109.5 \\
\hline $\mathrm{C}(21)-\mathrm{C}(22)-\mathrm{H}(22 \mathrm{C})$ & 109.5 \\
\hline $\mathrm{H}(22 \mathrm{~A})-\mathrm{C}(22)-\mathrm{H}(22 \mathrm{~B})$ & 109.5 \\
\hline $\mathrm{H}(22 \mathrm{~A})-\mathrm{C}(22)-\mathrm{H}(22 \mathrm{C})$ & 109.5 \\
\hline $\mathrm{H}(22 \mathrm{~B})-\mathrm{C}(22)-\mathrm{H}(22 \mathrm{C})$ & 109.5 \\
\hline $\mathrm{C}(7)-\mathrm{C}(8)-\mathrm{H}(8)$ & 119.6 \\
\hline $\mathrm{C}(9)-\mathrm{C}(8)-\mathrm{C}(7)$ & $120.8(2)$ \\
\hline $\mathrm{C}(9)-\mathrm{C}(8)-\mathrm{H}(8)$ & 119.6 \\
\hline $\mathrm{C}(61)-\mathrm{C}(62)-\mathrm{H}(62 \mathrm{~A})$ & 109.5 \\
\hline $\mathrm{C}(61)-\mathrm{C}(62)-\mathrm{H}(62 \mathrm{~B})$ & 109.5 \\
\hline $\mathrm{C}(61)-\mathrm{C}(62)-\mathrm{H}(62 \mathrm{C})$ & 109.5 \\
\hline$H(62 A)-C(62)-H(62 B)$ & 109.5 \\
\hline$H(62 A)-C(62)-H(62 C)$ & 109.5 \\
\hline $\mathrm{H}(62 \mathrm{~B})-\mathrm{C}(62)-\mathrm{H}(62 \mathrm{C})$ & 109.5 \\
\hline $\mathrm{C}(25)-\mathrm{C}(26)-\mathrm{H}(26 \mathrm{~A})$ & 109.5 \\
\hline $\mathrm{C}(25)-\mathrm{C}(26)-\mathrm{H}(26 \mathrm{~B})$ & 109.5 \\
\hline $\mathrm{C}(25)-\mathrm{C}(26)-\mathrm{H}(26 \mathrm{C})$ & 109.5 \\
\hline $\mathrm{H}(26 \mathrm{~A})-\mathrm{C}(26)-\mathrm{H}(26 \mathrm{~B})$ & 109.5 \\
\hline$H(26 A)-C(26)-H(26 C)$ & 109.5 \\
\hline$H(26 B)-C(26)-H(26 C)$ & 109.5 \\
\hline $\mathrm{C}(57)-\mathrm{C}(59)-\mathrm{H}(59 \mathrm{~A})$ & 109.5 \\
\hline
\end{tabular}




\begin{tabular}{|c|c|}
\hline $\mathrm{C}(57)-\mathrm{C}(59)-\mathrm{H}(59 \mathrm{~B})$ & 109.5 \\
\hline $\mathrm{C}(57)-\mathrm{C}(59)-\mathrm{H}(59 \mathrm{C})$ & 109.5 \\
\hline $\mathrm{H}(59 \mathrm{~A})-\mathrm{C}(59)-\mathrm{H}(59 \mathrm{~B})$ & 109.5 \\
\hline $\mathrm{H}(59 \mathrm{~A})-\mathrm{C}(59)-\mathrm{H}(59 \mathrm{C})$ & 109.5 \\
\hline $\mathrm{H}(59 \mathrm{~B})-\mathrm{C}(59)-\mathrm{H}(59 \mathrm{C})$ & 109.5 \\
\hline $\mathrm{C}(5)-\mathrm{C}(4)-\mathrm{H}(4)$ & 120.2 \\
\hline $\mathrm{C}(3)-\mathrm{C}(4)-\mathrm{C}(5)$ & $119.5(2)$ \\
\hline $\mathrm{C}(3)-\mathrm{C}(4)-\mathrm{H}(4)$ & 120.2 \\
\hline $\mathrm{C}(2)-\mathrm{C}(3)-\mathrm{H}(3)$ & 119.5 \\
\hline $\mathrm{C}(4)-\mathrm{C}(3)-\mathrm{C}(2)$ & $121.0(2)$ \\
\hline $\mathrm{C}(4)-\mathrm{C}(3)-\mathrm{H}(3)$ & 119.5 \\
\hline $\mathrm{C}(29)-\mathrm{C}(30)-\mathrm{H}(30 \mathrm{~A})$ & 109.5 \\
\hline $\mathrm{C}(29)-\mathrm{C}(30)-\mathrm{H}(30 \mathrm{~B})$ & 109.5 \\
\hline $\mathrm{C}(29)-\mathrm{C}(30)-\mathrm{H}(30 \mathrm{C})$ & 109.5 \\
\hline $\mathrm{H}(30 \mathrm{~A})-\mathrm{C}(30)-\mathrm{H}(30 \mathrm{~B})$ & 109.5 \\
\hline $\mathrm{H}(30 \mathrm{~A})-\mathrm{C}(30)-\mathrm{H}(30 \mathrm{C})$ & 109.5 \\
\hline $\mathrm{H}(30 \mathrm{~B})-\mathrm{C}(30)-\mathrm{H}(30 \mathrm{C})$ & 109.5 \\
\hline $\mathrm{C}(53)-\mathrm{C}(55)-\mathrm{H}(55 \mathrm{~A})$ & 109.5 \\
\hline $\mathrm{C}(53)-\mathrm{C}(55)-\mathrm{H}(55 \mathrm{~B})$ & 109.5 \\
\hline $\mathrm{C}(53)-\mathrm{C}(55)-\mathrm{H}(55 \mathrm{C})$ & 109.5 \\
\hline $\mathrm{H}(55 \mathrm{~A})-\mathrm{C}(55)-\mathrm{H}(55 \mathrm{~B})$ & 109.5 \\
\hline $\mathrm{H}(55 \mathrm{~A})-\mathrm{C}(55)-\mathrm{H}(55 \mathrm{C})$ & 109.5 \\
\hline $\mathrm{H}(55 \mathrm{~B})-\mathrm{C}(55)-\mathrm{H}(55 \mathrm{C})$ & 109.5 \\
\hline $\mathrm{C}(29)-\mathrm{C}(32)-\mathrm{H}(32 \mathrm{~A})$ & 109.5 \\
\hline $\mathrm{C}(29)-\mathrm{C}(32)-\mathrm{H}(32 \mathrm{~B})$ & 109.5 \\
\hline $\mathrm{C}(29)-\mathrm{C}(32)-\mathrm{H}(32 \mathrm{C})$ & 109.5 \\
\hline $\mathrm{H}(32 \mathrm{~A})-\mathrm{C}(32)-\mathrm{H}(32 \mathrm{~B})$ & 109.5 \\
\hline $\mathrm{H}(32 \mathrm{~A})-\mathrm{C}(32)-\mathrm{H}(32 \mathrm{C})$ & 109.5 \\
\hline $\mathrm{H}(32 \mathrm{~B})-\mathrm{C}(32)-\mathrm{H}(32 \mathrm{C})$ & 109.5 \\
\hline $\mathrm{C}(29)-\mathrm{C}(31)-\mathrm{H}(31 \mathrm{~A})$ & 109.5 \\
\hline $\mathrm{C}(29)-\mathrm{C}(31)-\mathrm{H}(31 \mathrm{~B})$ & 109.5 \\
\hline $\mathrm{C}(29)-\mathrm{C}(31)-\mathrm{H}(31 \mathrm{C})$ & 109.5 \\
\hline $\mathrm{H}(31 \mathrm{~A})-\mathrm{C}(31)-\mathrm{H}(31 \mathrm{~B})$ & 109.5 \\
\hline $\mathrm{H}(31 \mathrm{~A})-\mathrm{C}(31)-\mathrm{H}(31 \mathrm{C})$ & 109.5 \\
\hline $\mathrm{H}(31 \mathrm{~B})-\mathrm{C}(31)-\mathrm{H}(31 \mathrm{C})$ & 109.5 \\
\hline $\mathrm{C}(53)-\mathrm{C}(54)-\mathrm{H}(54 \mathrm{~A})$ & 109.5 \\
\hline
\end{tabular}




$\begin{array}{ll}\text { C(53)-C(54)-H(54B) } & 109.5 \\ \text { C(53)-C(54)-H(54C) } & 109.5 \\ \text { H(54A)-C(54)-H(54B) } & 109.5 \\ \text { H(54A)-C(54)-H(54C) } & 109.5 \\ \text { H(54B)-C(54)-H(54C) } & 109.5\end{array}$

Symmetry transformations used to generate equivalent atoms: 
Table S9. Anisotropic displacement parameters $\left(\AA^{2} \times 10^{3}\right)$ for cain09_a. The anisotropic displacement factor exponent takes the form: $-2 \pi^{2}\left[h^{2} a^{* 2} U^{11}+\ldots+2 h k a^{*} b^{*} U^{12}\right]$

\begin{tabular}{|c|c|c|c|c|c|c|}
\hline & $\mathrm{U}^{11}$ & $\mathrm{U}^{22}$ & $\mathrm{U}^{33}$ & $\mathrm{U}^{23}$ & $\mathrm{U}^{13}$ & $\mathrm{U}^{12}$ \\
\hline $\mathrm{P}(3)$ & $29(1)$ & $28(1)$ & $24(1)$ & $5(1)$ & $6(1)$ & $12(1)$ \\
\hline $\mathrm{P}(4)$ & $42(1)$ & $34(1)$ & $28(1)$ & $13(1)$ & $13(1)$ & $21(1)$ \\
\hline $\mathrm{P}(2)$ & $39(1)$ & $25(1)$ & $26(1)$ & $6(1)$ & $4(1)$ & $11(1)$ \\
\hline $\mathrm{P}(1)$ & $36(1)$ & $28(1)$ & $23(1)$ & $6(1)$ & $5(1)$ & $12(1)$ \\
\hline $\mathrm{C}(19)$ & $34(2)$ & $29(1)$ & $31(1)$ & $9(1)$ & $11(1)$ & $16(1)$ \\
\hline $\mathrm{C}(48)$ & $32(1)$ & $35(1)$ & $19(1)$ & 11(1) & $9(1)$ & $14(1)$ \\
\hline $\mathrm{C}(18)$ & $29(1)$ & $26(1)$ & $25(1)$ & $7(1)$ & $9(1)$ & $9(1)$ \\
\hline $\mathrm{C}(49)$ & $31(1)$ & $27(1)$ & $25(1)$ & $8(1)$ & $6(1)$ & $9(1)$ \\
\hline$C(15)$ & $32(1)$ & $26(1)$ & $22(1)$ & $7(1)$ & $6(1)$ & $10(1)$ \\
\hline$C(52)$ & $33(1)$ & $28(1)$ & $19(1)$ & $6(1)$ & $7(1)$ & $12(1)$ \\
\hline $\mathrm{C}(53)$ & $29(1)$ & $37(2)$ & $29(1)$ & $10(1)$ & $7(1)$ & $13(1)$ \\
\hline $\mathrm{C}(61)$ & $33(1)$ & $26(1)$ & $25(1)$ & $2(1)$ & $7(1)$ & $7(1)$ \\
\hline $\mathrm{C}(47)$ & $32(1)$ & $31(1)$ & $21(1)$ & $10(1)$ & $7(1)$ & $13(1)$ \\
\hline $\mathrm{C}(25)$ & $32(1)$ & $25(1)$ & $26(1)$ & $2(1)$ & $5(1)$ & $9(1)$ \\
\hline $\mathrm{C}(17)$ & $24(1)$ & $32(1)$ & $22(1)$ & $4(1)$ & $4(1)$ & $9(1)$ \\
\hline $\mathrm{C}(20)$ & $30(1)$ & $28(1)$ & $21(1)$ & $8(1)$ & $6(1)$ & $10(1)$ \\
\hline $\mathrm{C}(50)$ & $34(2)$ & $29(1)$ & $20(1)$ & $8(1)$ & $9(1)$ & $13(1)$ \\
\hline $\mathrm{C}(43)$ & $38(2)$ & $27(1)$ & $37(2)$ & $8(1)$ & $14(1)$ & $11(1)$ \\
\hline C(44) & $29(1)$ & $29(1)$ & $25(1)$ & $2(1)$ & $7(1)$ & $9(1)$ \\
\hline $\mathrm{C}(21)$ & $31(1)$ & $35(1)$ & $27(1)$ & $7(1)$ & $8(1)$ & $16(1)$ \\
\hline$C(16)$ & $32(1)$ & $31(1)$ & $20(1)$ & $8(1)$ & $8(1)$ & $13(1)$ \\
\hline$C(7)$ & $35(2)$ & $27(1)$ & $22(1)$ & $2(1)$ & $2(1)$ & $9(1)$ \\
\hline $\mathrm{C}(27)$ & $52(2)$ & $29(1)$ & $31(1)$ & $2(1)$ & $5(1)$ & $16(1)$ \\
\hline $\mathrm{C}(57)$ & $34(2)$ & $30(1)$ & $30(1)$ & $8(1)$ & 11(1) & $15(1)$ \\
\hline $\mathrm{C}(38)$ & $30(1)$ & $34(1)$ & $29(1)$ & $8(1)$ & $7(1)$ & $14(1)$ \\
\hline $\mathrm{C}(12)$ & $37(2)$ & $39(2)$ & $28(1)$ & $4(1)$ & $4(1)$ & $13(1)$ \\
\hline$C(6)$ & $42(2)$ & $34(2)$ & $26(1)$ & $7(1)$ & $5(1)$ & $13(1)$ \\
\hline$C(46)$ & $25(1)$ & $28(1)$ & $29(1)$ & $9(1)$ & $4(1)$ & $11(1)$ \\
\hline$C(36)$ & $26(1)$ & $31(1)$ & $31(1)$ & $-2(1)$ & $5(1)$ & $9(1)$ \\
\hline$C(29)$ & $28(1)$ & $25(1)$ & $38(1)$ & $9(1)$ & $4(1)$ & $8(1)$ \\
\hline $\mathrm{C}(37)$ & $32(2)$ & $40(2)$ & $26(1)$ & $5(1)$ & $9(1)$ & $13(1)$ \\
\hline
\end{tabular}




\begin{tabular}{|c|c|c|c|c|c|c|}
\hline $\mathrm{C}(33)$ & $18(1)$ & $27(1)$ & $28(1)$ & $4(1)$ & $3(1)$ & $5(1)$ \\
\hline $\mathrm{C}(41)$ & $29(1)$ & $38(2)$ & $28(1)$ & $9(1)$ & $6(1)$ & $14(1)$ \\
\hline $\mathrm{C}(42)$ & $36(2)$ & $35(1)$ & $30(1)$ & $13(1)$ & $12(1)$ & $18(1)$ \\
\hline $\mathrm{C}(13)$ & $43(2)$ & $30(1)$ & $26(1)$ & $4(1)$ & $-1(1)$ & $14(1)$ \\
\hline C(39) & $26(1)$ & $28(1)$ & $26(1)$ & $5(1)$ & $10(1)$ & 11(1) \\
\hline $\mathrm{C}(40)$ & $28(1)$ & $28(1)$ & $30(1)$ & $6(1)$ & $8(1)$ & 11(1) \\
\hline $\mathrm{C}(5)$ & $51(2)$ & $31(2)$ & $37(2)$ & $8(1)$ & $4(1)$ & $17(1)$ \\
\hline $\mathrm{C}(51)$ & $28(1)$ & $33(1)$ & $22(1)$ & $7(1)$ & $7(1)$ & 11(1) \\
\hline $\mathrm{C}(9)$ & $51(2)$ & $34(2)$ & $31(1)$ & $9(1)$ & $1(1)$ & $14(1)$ \\
\hline$C(28)$ & $57(2)$ & $25(1)$ & $41(2)$ & $5(1)$ & $-9(1)$ & $9(1)$ \\
\hline $\mathrm{C}(60)$ & $43(2)$ & $31(1)$ & $40(2)$ & $10(1)$ & $12(1)$ & $16(1)$ \\
\hline$C(34)$ & $27(1)$ & $32(1)$ & $28(1)$ & $7(1)$ & $4(1)$ & $10(1)$ \\
\hline $\mathrm{C}(58)$ & $41(2)$ & $36(2)$ & $52(2)$ & $9(1)$ & $7(1)$ & $19(1)$ \\
\hline$C(1)$ & $31(1)$ & $30(1)$ & $26(1)$ & $5(1)$ & $3(1)$ & $13(1)$ \\
\hline$C(35)$ & $32(2)$ & $27(1)$ & $38(2)$ & $7(1)$ & $8(1)$ & $12(1)$ \\
\hline$C(24)$ & $31(2)$ & $39(2)$ & $30(1)$ & $6(1)$ & $9(1)$ & $14(1)$ \\
\hline$C(23)$ & $46(2)$ & $46(2)$ & $47(2)$ & $22(1)$ & $18(1)$ & $28(2)$ \\
\hline $\mathrm{C}(11)$ & $39(2)$ & $45(2)$ & $30(1)$ & $7(1)$ & $10(1)$ & $5(1)$ \\
\hline $\mathrm{C}(45)$ & $32(1)$ & $28(1)$ & $27(1)$ & $7(1)$ & $9(1)$ & $14(1)$ \\
\hline$C(2)$ & $55(2)$ & $33(2)$ & $28(1)$ & $8(1)$ & $9(1)$ & 11(1) \\
\hline$C(10)$ & $55(2)$ & $34(2)$ & $24(1)$ & $7(1)$ & $4(1)$ & $4(1)$ \\
\hline$C(64)$ & $49(2)$ & $28(1)$ & $36(2)$ & $10(1)$ & $6(1)$ & $6(1)$ \\
\hline$C(56)$ & $32(2)$ & $60(2)$ & $45(2)$ & $19(2)$ & $14(1)$ & $9(2)$ \\
\hline $\mathrm{C}(14)$ & $34(2)$ & $26(1)$ & $30(1)$ & $9(1)$ & $1(1)$ & $12(1)$ \\
\hline $\mathrm{C}(63)$ & $40(2)$ & $31(2)$ & $45(2)$ & $3(1)$ & $17(1)$ & $6(1)$ \\
\hline$C(22)$ & $40(2)$ & $50(2)$ & $36(2)$ & $2(1)$ & $2(1)$ & $28(2)$ \\
\hline $\mathrm{C}(8)$ & $38(2)$ & $36(2)$ & $26(1)$ & $6(1)$ & $3(1)$ & $13(1)$ \\
\hline$C(62)$ & $32(2)$ & $39(2)$ & $28(1)$ & $1(1)$ & $2(1)$ & $7(1)$ \\
\hline$C(26)$ & $39(2)$ & $30(2)$ & $77(2)$ & $-6(2)$ & $22(2)$ & $-1(1)$ \\
\hline C(59) & $61(2)$ & $42(2)$ & $42(2)$ & $14(1)$ & $26(2)$ & $29(2)$ \\
\hline $\mathrm{C}(4)$ & $51(2)$ & $34(2)$ & $32(2)$ & $-3(1)$ & $-1(1)$ & $18(1)$ \\
\hline$C(3)$ & $64(2)$ & $46(2)$ & $27(1)$ & $5(1)$ & $10(1)$ & $18(2)$ \\
\hline $\mathrm{C}(30)$ & $44(2)$ & $76(2)$ & $38(2)$ & $7(2)$ & $-3(1)$ & $34(2)$ \\
\hline$C(55)$ & $33(2)$ & $75(2)$ & $96(3)$ & $52(2)$ & $5(2)$ & $20(2)$ \\
\hline$C(32)$ & $31(2)$ & $45(2)$ & $64(2)$ & $6(2)$ & $14(2)$ & $12(1)$ \\
\hline $\mathrm{C}(31)$ & $28(2)$ & $52(2)$ & $110(3)$ & $45(2)$ & $11(2)$ & $7(2)$ \\
\hline
\end{tabular}


C(54)

30(2)

$80(2)$

60(2)

$-24(2)$

$-2(2)$

14(2) 
Table S10. Hydrogen coordinates ( x 104) and isotropic displacement parameters $\left(\AA^{2} \mathrm{x} 10^{3}\right)$ for cain09_a.

\begin{tabular}{|c|c|c|c|c|}
\hline & $\mathrm{x}$ & $\mathrm{y}$ & $\mathrm{z}$ & $\mathrm{U}(\mathrm{eq})$ \\
\hline $\mathrm{H}(19)$ & 4704 & 9876 & 3420 & 36 \\
\hline $\mathrm{H}(49)$ & 5293 & 9916 & 8484 & 34 \\
\hline $\mathrm{H}(17)$ & 8459 & 9676 & 3811 & 33 \\
\hline $\mathrm{H}(43)$ & 7867 & 9665 & 5585 & 41 \\
\hline $\mathrm{H}(44)$ & 8321 & 8744 & 6312 & 35 \\
\hline $\mathrm{H}(27 \mathrm{~A})$ & 6301 & 10993 & 4739 & 58 \\
\hline $\mathrm{H}(27 \mathrm{~B})$ & 7692 & 12014 & 4946 & 58 \\
\hline $\mathrm{H}(27 \mathrm{C})$ & 7814 & 10944 & 4936 & 58 \\
\hline $\mathrm{H}(38)$ & 7567 & 6355 & 4982 & 37 \\
\hline $\mathrm{H}(12)$ & 4872 & 6115 & 267 & 44 \\
\hline $\mathrm{H}(6)$ & 1341 & 3244 & 778 & 42 \\
\hline $\mathrm{H}(36)$ & 8620 & 3904 & 4680 & 38 \\
\hline $\mathrm{H}(37)$ & 8175 & 5186 & 4303 & 40 \\
\hline $\mathrm{H}(41)$ & 4426 & 7200 & 4491 & 37 \\
\hline $\mathrm{H}(42)$ & 5915 & 8899 & 4673 & 38 \\
\hline $\mathrm{H}(13 \mathrm{~A})$ & 3760 & 4800 & 1541 & 41 \\
\hline $\mathrm{H}(13 \mathrm{~B})$ & 4654 & 5311 & 1061 & 41 \\
\hline $\mathrm{H}(40)$ & 4893 & 6265 & 5207 & 34 \\
\hline $\mathrm{H}(5)$ & 586 & 1674 & -11 & 49 \\
\hline $\mathrm{H}(51)$ & 1352 & 7856 & 8079 & 34 \\
\hline $\mathrm{H}(9)$ & 2189 & 8002 & -172 & 49 \\
\hline $\mathrm{H}(28 \mathrm{~A})$ & 6537 & 11522 & 3180 & 69 \\
\hline $\mathrm{H}(28 \mathrm{~B})$ & 7062 & 12415 & 3861 & 69 \\
\hline $\mathrm{H}(28 \mathrm{C})$ & 5614 & 11455 & 3720 & 69 \\
\hline $\mathrm{H}(60 \mathrm{~A})$ & 4622 & 11109 & 8799 & 56 \\
\hline $\mathrm{H}(60 \mathrm{~B})$ & 3360 & 11439 & 8554 & 56 \\
\hline $\mathrm{H}(60 \mathrm{C})$ & 3980 & 10885 & 8019 & 56 \\
\hline $\mathrm{H}(34)$ & 7797 & 4940 & 6434 & 36 \\
\hline $\mathrm{H}(58 \mathrm{~A})$ & 1551 & 9662 & 7491 & 65 \\
\hline $\mathrm{H}(58 \mathrm{~B})$ & 979 & 10193 & 8060 & 65 \\
\hline
\end{tabular}




\begin{tabular}{|c|c|c|c|}
\hline $\mathrm{H}(58 \mathrm{C})$ & 637 & 8998 & 7902 \\
\hline $\mathrm{H}(35)$ & 8444 & 3795 & 5754 \\
\hline $\mathrm{H}(24 \mathrm{~A})$ & 7364 & 7004 & 2394 \\
\hline $\mathrm{H}(24 \mathrm{~B})$ & 8885 & 7112 & 2769 \\
\hline $\mathrm{H}(24 \mathrm{C})$ & 8662 & 8082 & 2606 \\
\hline $\mathrm{H}(23 \mathrm{~A})$ & 6882 & 6894 & 4014 \\
\hline $\mathrm{H}(23 \mathrm{~B})$ & 8006 & 6502 & 3745 \\
\hline $\mathrm{H}(23 \mathrm{C})$ & 6510 & 6224 & 3259 \\
\hline $\mathrm{H}(11)$ & 5622 & 7367 & -284 \\
\hline $\mathrm{H}(45 \mathrm{~A})$ & 4839 & 5512 & 6033 \\
\hline $\mathrm{H}(45 \mathrm{~B})$ & 5640 & 5338 & 6682 \\
\hline $\mathrm{H}(2)$ & 2273 & 4672 & -642 \\
\hline $\mathrm{H}(10)$ & 4291 & 8315 & -501 \\
\hline $\mathrm{H}(64 \mathrm{~A})$ & 2908 & 5401 & 8116 \\
\hline $\mathrm{H}(64 \mathrm{~B})$ & 1300 & 4810 & 8113 \\
\hline $\mathrm{H}(64 \mathrm{C})$ & 2256 & 5840 & 8685 \\
\hline $\mathrm{H}(56 \mathrm{~A})$ & 6903 & 8452 & 7378 \\
\hline $\mathrm{H}(56 \mathrm{~B})$ & 8300 & 9376 & 7840 \\
\hline $\mathrm{H}(56 \mathrm{C})$ & 6903 & 9570 & 7614 \\
\hline $\mathrm{H}(63 \mathrm{~A})$ & 354 & 6470 & 8382 \\
\hline $\mathrm{H}(63 \mathrm{~B})$ & -468 & 5403 & 7824 \\
\hline $\mathrm{H}(63 \mathrm{C})$ & -170 & 6449 & 7624 \\
\hline $\mathrm{H}(22 \mathrm{~A})$ & 9771 & 8979 & 3785 \\
\hline $\mathrm{H}(22 \mathrm{~B})$ & 9848 & 7984 & 3973 \\
\hline $\mathrm{H}(22 \mathrm{C})$ & 9079 & 8614 & 4361 \\
\hline $\mathrm{H}(8)$ & 1438 & 6764 & 390 \\
\hline $\mathrm{H}(62 \mathrm{~A})$ & 1037 & 5963 & 6766 \\
\hline $\mathrm{H}(62 \mathrm{~B})$ & 530 & 4886 & 6931 \\
\hline $\mathrm{H}(62 \mathrm{C})$ & 2145 & 5462 & 6938 \\
\hline $\mathrm{H}(26 \mathrm{~A})$ & 9442 & 11273 & 4200 \\
\hline $\mathrm{H}(26 \mathrm{~B})$ & 9288 & 12330 & 4200 \\
\hline $\mathrm{H}(26 \mathrm{C})$ & 8981 & 11499 & 3509 \\
\hline $\mathrm{H}(59 \mathrm{~A})$ & 1655 & 9296 & 9127 \\
\hline $\mathrm{H}(59 \mathrm{~B})$ & 2064 & 10498 & 9262 \\
\hline $\mathrm{H}(59 \mathrm{C})$ & 3246 & 10073 & 9467 \\
\hline $\mathrm{H}(4)$ & 626 & 1604 & -1120 \\
\hline
\end{tabular}




\begin{tabular}{lllll}
$\mathrm{H}(3)$ & 1469 & 3099 & -1425 & 57 \\
$\mathrm{H}(30 \mathrm{~A})$ & 3003 & 8431 & 1992 & 79 \\
$\mathrm{H}(30 \mathrm{~B})$ & 1500 & 7604 & 1966 & 79 \\
$\mathrm{H}(30 \mathrm{C})$ & 2785 & 7262 & 1896 & 79 \\
$\mathrm{H}(55 \mathrm{~A})$ & 7001 & 7840 & 8908 & 97 \\
$\mathrm{H}(55 \mathrm{~B})$ & 8453 & 8464 & 8752 & 97 \\
$\mathrm{H}(55 \mathrm{C})$ & 7249 & 7539 & 8179 & 97 \\
$\mathrm{H}(32 \mathrm{~A})$ & 2669 & 8962 & 3663 & 73 \\
$\mathrm{H}(32 \mathrm{~B})$ & 1317 & 8458 & 3045 & 73 \\
$\mathrm{H}(32 \mathrm{C})$ & 2676 & 9379 & 3018 & 73 \\
$\mathrm{H}(31 \mathrm{~A})$ & 2390 & 6486 & 2845 & 94 \\
$\mathrm{H}(31 \mathrm{~B})$ & 1178 & 6886 & 2972 & 94 \\
$\mathrm{H}(31 \mathrm{C})$ & 2538 & 7192 & 3562 & 94 \\
$\mathrm{H}(54 \mathrm{~A})$ & 7324 & 10386 & 8792 & 101 \\
$\mathrm{H}(54 \mathrm{~B})$ & 8519 & 10011 & 9064 & 101 \\
$\mathrm{H}(54 \mathrm{C})$ & 7105 & 9732 & 9320 & 101 \\
$\mathrm{H}(14)$ & $5070(20)$ & $6951(18)$ & $1732(11)$ & $27(6)$ \\
$\mathrm{H}(46)$ & $4650(20)$ & $6970(17)$ & $6736(11)$ & $23(6)$ \\
\hline & & & & \\
\hline
\end{tabular}


Table S11. Hydrogen bonds for cain09_a [ $\AA$ and $\left.{ }^{\circ}\right]$.

$\begin{array}{lllll}\mathrm{D}-\mathrm{H} . . . \mathrm{A} & \mathrm{d}(\mathrm{D}-\mathrm{H}) & \mathrm{d}(\mathrm{H} \ldots \mathrm{A}) & \mathrm{d}(\mathrm{D} \ldots \mathrm{A}) & <(\mathrm{DHA})\end{array}$




\section{Data for 2a}

Table S12. Crystal data and structure refinement for CAIN03 (2a).

Identification code

Empirical formula

Formula weight

Temperature

Wavelength

Crystal system

Space group

Unit cell dimensions

Volume

$\mathrm{Z}$

Density (calculated)

Absorption coefficient

$\mathrm{F}(000)$

Crystal size

Theta range for data collection

Index ranges

Reflections collected

Independent reflections

Completeness to theta $=25.000^{\circ}$

Absorption correction

Refinement method

Data / restraints / parameters

Goodness-of-fit on $\mathrm{F}^{2}$

Final $\mathrm{R}$ indices [I $>2 \operatorname{sigma}(\mathrm{I})]$

$\mathrm{R}$ indices (all data)

Extinction coefficient

Largest diff. peak and hole
CAIN03

C33.31 H55.31 Cl5.93 P2 Pd

834.36

100(2) K

$0.71073 \AA$

Monoclinic

P 21/c

$\mathrm{a}=14.5058(6) \AA$

$\alpha=90^{\circ}$.

$\mathrm{b}=15.1344(7) \AA$

$\beta=106.901(3)^{\circ}$.

$\mathrm{c}=19.3313(11) \AA$ $\gamma=90^{\circ}$.

4060.6(3) $\AA^{3}$

4

$1.365 \mathrm{Mg} / \mathrm{m}^{3}$

$0.947 \mathrm{~mm}^{-1}$

1728

$0.330 \times 0.140 \times 0.100 \mathrm{~mm}^{3}$

2.202 to $28.291^{\circ}$.

$-19<=\mathrm{h}<=19,-20<=\mathrm{k}<=20,-25<=1<=25$

33925

$10046[\mathrm{R}($ int $)=0.0776]$

$99.9 \%$

Multi-scan

Full-matrix least-squares on $\mathrm{F}^{2}$

10046 / 6 / 409

1.038

$\mathrm{R} 1=0.0637, \mathrm{wR} 2=0.1499$

$\mathrm{R} 1=0.1091, \mathrm{wR} 2=0.1757$

$\mathrm{n} / \mathrm{a}$

2.680 and -1.039 e. $\AA^{-3}$ 
Table S13. Atomic coordinates $\left(\times 10^{4}\right)$ and equivalent isotropic displacement parameters $\left(\AA^{2} \times 10^{3}\right)$ for CAIN03. U(eq) is defined as one third of the trace of the orthogonalized $\mathrm{U}^{\mathrm{ij}}$ tensor.

\begin{tabular}{|c|c|c|c|c|}
\hline & $\mathrm{x}$ & $\mathrm{y}$ & $\mathrm{z}$ & $\mathrm{U}(\mathrm{eq})$ \\
\hline $\operatorname{Pd}(1)$ & $8666(1)$ & $3420(1)$ & 7411(1) & $15(1)$ \\
\hline $\mathrm{P}(1)$ & $9422(1)$ & $2180(1)$ & 7929(1) & $17(1)$ \\
\hline $\mathrm{P}(2)$ & $7426(1)$ & $2855(1)$ & 7715(1) & 19(1) \\
\hline $\mathrm{Cl}(1)$ & $10090(1)$ & $3915(1)$ & 7193(1) & $25(1)$ \\
\hline $\mathrm{Cl}(2)$ & 7821(1) & $4722(1)$ & 6906(1) & $24(1)$ \\
\hline $\mathrm{Cl}(3)$ & $9738(4)$ & 4681(3) & 5218(3) & $108(2)$ \\
\hline $\mathrm{Cl}(4)$ & 8866(3) & $3019(2)$ & 5415(2) & $69(1)$ \\
\hline $\mathrm{Cl}(5)$ & $7716(4)$ & $4507(3)$ & 4841(2) & $112(3)$ \\
\hline $\mathrm{Cl}(6)$ & 4153(3) & $3640(3)$ & 4071(2) & $94(2)$ \\
\hline $\mathrm{Cl}(7)$ & $4950(2)$ & $3957(2)$ & 2916(2) & $70(1)$ \\
\hline $\mathrm{Cl}(8)$ & $5560(2)$ & 4992(2) & 4219(2) & 74(1) \\
\hline $\mathrm{C}(1)$ & $8670(3)$ & 1588(3) & 8414(3) & 21(1) \\
\hline$C(2)$ & 7667(3) & 1950(3) & $8208(3)$ & 19(1) \\
\hline$C(3)$ & $9626(3)$ & 1349(3) & 7300(3) & 19(1) \\
\hline $\mathrm{C}(4)$ & $8676(4)$ & $1158(4)$ & 6711(3) & $23(1)$ \\
\hline$C(5)$ & $8815(4)$ & $454(4)$ & 6184(3) & $26(1)$ \\
\hline$C(6)$ & $9608(4)$ & 709(4) & 5851(3) & $28(1)$ \\
\hline$C(7)$ & $10551(4)$ & $870(4)$ & 6439(3) & $27(1)$ \\
\hline $\mathrm{C}(8)$ & $10435(3)$ & $1590(3)$ & 6973(3) & $21(1)$ \\
\hline $\mathrm{C}(9)$ & $10567(3)$ & $2426(3)$ & 8609(3) & $21(1)$ \\
\hline$C(10)$ & $11077(4)$ & $1600(4)$ & 9006(3) & $29(1)$ \\
\hline $\mathrm{C}(11)$ & $12015(4)$ & $1854(5)$ & 9578(4) & $40(2)$ \\
\hline $\mathrm{C}(12)$ & $11823(4)$ & $2522(4)$ & $10115(3)$ & $35(2)$ \\
\hline$C(13)$ & $11350(4)$ & $3346(4)$ & 9715(3) & $28(1)$ \\
\hline$C(14)$ & $10404(3)$ & $3113(3)$ & 9148(3) & 21(1) \\
\hline$C(15)$ & $6189(3)$ & $3217(3)$ & 7596(3) & 20(1) \\
\hline$C(16)$ & $5432(4)$ & 2935(3) & 6970(3) & $22(1)$ \\
\hline $\mathrm{C}(17)$ & 4495(3) & $3104(4)$ & 6975(3) & $24(1)$ \\
\hline $\mathrm{C}(18)$ & 4249(3) & $3518(3)$ & 7539(3) & $22(1)$ \\
\hline $\mathrm{C}(19)$ & 4998(4) & $3811(4)$ & 8114(3) & $25(1)$ \\
\hline $\mathrm{C}(20)$ & 5972(3) & $3690(4)$ & 8163(3) & 21(1) \\
\hline
\end{tabular}




\begin{tabular}{lllll}
$\mathrm{C}(21)$ & $6290(4)$ & $2980(5)$ & $5989(3)$ & $40(2)$ \\
$\mathrm{C}(22)$ & $5953(4)$ & $1495(4)$ & $6486(4)$ & $43(2)$ \\
$\mathrm{C}(23)$ & $4643(4)$ & $2367(4)$ & $5689(3)$ & $36(2)$ \\
$\mathrm{C}(24)$ & $5589(4)$ & $2453(4)$ & $6303(3)$ & $30(1)$ \\
$\mathrm{C}(25)$ & $3060(4)$ & $4014(5)$ & $8200(4)$ & $48(2)$ \\
$\mathrm{C}(26)$ & $2730(4)$ & $4295(5)$ & $6883(4)$ & $49(2)$ \\
$\mathrm{C}(27)$ & $2649(4)$ & $2771(4)$ & $7344(4)$ & $40(2)$ \\
$\mathrm{C}(28)$ & $3191(4)$ & $3652(4)$ & $7501(3)$ & $29(1)$ \\
$\mathrm{C}(29)$ & $6331(6)$ & $4913(7)$ & $9089(7)$ & $132(6)$ \\
$\mathrm{C}(30)$ & $6786(8)$ & $3383(7)$ & $9468(4)$ & $95(4)$ \\
$\mathrm{C}(31)$ & $7706(5)$ & $4261(6)$ & $8832(4)$ & $53(2)$ \\
$\mathrm{C}(32)$ & $6704(4)$ & $4066(4)$ & $8862(3)$ & $31(1)$ \\
$\mathrm{C}(33)$ & $8795(3)$ & $4161(2)$ & $5436(4)$ & $34(3)$ \\
$\mathrm{C}(34)$ & $4616(4)$ & $4436(3)$ & $3621(2)$ & $38(2)$ \\
\hline
\end{tabular}


Table S14. Bond lengths $[\AA]$ and angles $\left[{ }^{\circ}\right]$ for CAIN03.

\begin{tabular}{|c|c|}
\hline $\mathrm{Pd}(1)-\mathrm{P}(2)$ & $2.2194(14)$ \\
\hline $\mathrm{Pd}(1)-\mathrm{P}(1)$ & $2.2558(13)$ \\
\hline $\operatorname{Pd}(1)-\mathrm{Cl}(1)$ & $2.3485(13)$ \\
\hline $\mathrm{Pd}(1)-\mathrm{Cl}(2)$ & $2.3748(13)$ \\
\hline $\mathrm{P}(1)-\mathrm{C}(9)$ & $1.832(5)$ \\
\hline $\mathrm{P}(1)-\mathrm{C}(3)$ & $1.833(5)$ \\
\hline $\mathrm{P}(1)-\mathrm{C}(1)$ & $1.863(5)$ \\
\hline $\mathrm{P}(2)-\mathrm{C}(2)$ & $1.648(5)$ \\
\hline$P(2)-C(15)$ & $1.825(5)$ \\
\hline $\mathrm{Cl}(3)-\mathrm{Cl}(3) \# 1$ & $1.611(9)$ \\
\hline $\mathrm{Cl}(3)-\mathrm{C}(33)$ & $1.732(3)$ \\
\hline $\mathrm{Cl}(4)-\mathrm{C}(33)$ & $1.732(3)$ \\
\hline $\mathrm{Cl}(5)-\mathrm{C}(33)$ & $1.732(3)$ \\
\hline $\mathrm{Cl}(6)-\mathrm{C}(34)$ & $1.732(2)$ \\
\hline $\mathrm{Cl}(7)-\mathrm{C}(34)$ & $1.733(2)$ \\
\hline $\mathrm{Cl}(8)-\mathrm{C}(34)$ & $1.732(2)$ \\
\hline $\mathrm{C}(1)-\mathrm{C}(2)$ & $1.495(6)$ \\
\hline $\mathrm{C}(3)-\mathrm{C}(8)$ & $1.531(7)$ \\
\hline $\mathrm{C}(3)-\mathrm{C}(4)$ & $1.538(7)$ \\
\hline $\mathrm{C}(4)-\mathrm{C}(5)$ & $1.529(7)$ \\
\hline $\mathrm{C}(5)-\mathrm{C}(6)$ & $1.522(8)$ \\
\hline$C(6)-C(7)$ & $1.522(7)$ \\
\hline $\mathrm{C}(7)-\mathrm{C}(8)$ & $1.543(7)$ \\
\hline $\mathrm{C}(9)-\mathrm{C}(14)$ & $1.537(7)$ \\
\hline $\mathrm{C}(9)-\mathrm{C}(10)$ & $1.539(7)$ \\
\hline $\mathrm{C}(10)-\mathrm{C}(11)$ & $1.533(8)$ \\
\hline $\mathrm{C}(11)-\mathrm{C}(12)$ & $1.531(9)$ \\
\hline $\mathrm{C}(12)-\mathrm{C}(13)$ & $1.522(8)$ \\
\hline $\mathrm{C}(13)-\mathrm{C}(14)$ & $1.528(7)$ \\
\hline$C(15)-C(20)$ & $1.419(8)$ \\
\hline$C(15)-C(16)$ & $1.442(7)$ \\
\hline $\mathrm{C}(16)-\mathrm{C}(17)$ & $1.387(7)$ \\
\hline$C(16)-C(24)$ & $1.554(8)$ \\
\hline $\mathrm{C}(17)-\mathrm{C}(18)$ & $1.391(8)$ \\
\hline
\end{tabular}




\begin{tabular}{|c|c|}
\hline$C(18)-C(19)$ & $1.383(7)$ \\
\hline $\mathrm{C}(18)-\mathrm{C}(28)$ & $1.528(7)$ \\
\hline$C(19)-C(20)$ & $1.400(7)$ \\
\hline$C(20)-C(32)$ & $1.563(7)$ \\
\hline$C(21)-C(24)$ & $1.548(9)$ \\
\hline$C(22)-C(24)$ & $1.549(9)$ \\
\hline$C(23)-C(24)$ & $1.536(7)$ \\
\hline$C(25)-C(28)$ & $1.521(9)$ \\
\hline$C(26)-C(28)$ & $1.535(9)$ \\
\hline $\mathrm{C}(27)-\mathrm{C}(28)$ & $1.533(8)$ \\
\hline$C(29)-C(32)$ & $1.505(10)$ \\
\hline$C(30)-C(32)$ & $1.541(11)$ \\
\hline $\mathrm{C}(31)-\mathrm{C}(32)$ & $1.500(8)$ \\
\hline $\mathrm{P}(2)-\mathrm{Pd}(1)-\mathrm{P}(1)$ & $83.65(5)$ \\
\hline $\mathrm{P}(2)-\mathrm{Pd}(1)-\mathrm{Cl}(1)$ & $173.50(5)$ \\
\hline $\mathrm{P}(1)-\mathrm{Pd}(1)-\mathrm{Cl}(1)$ & $90.33(5)$ \\
\hline $\mathrm{P}(2)-\mathrm{Pd}(1)-\mathrm{Cl}(2)$ & $93.83(5)$ \\
\hline $\mathrm{P}(1)-\mathrm{Pd}(1)-\mathrm{Cl}(2)$ & $177.10(5)$ \\
\hline $\mathrm{Cl}(1)-\mathrm{Pd}(1)-\mathrm{Cl}(2)$ & $92.12(5)$ \\
\hline $\mathrm{C}(9)-\mathrm{P}(1)-\mathrm{C}(3)$ & 109.2(2) \\
\hline $\mathrm{C}(9)-\mathrm{P}(1)-\mathrm{C}(1)$ & $106.2(3)$ \\
\hline $\mathrm{C}(3)-\mathrm{P}(1)-\mathrm{C}(1)$ & $103.6(2)$ \\
\hline $\mathrm{C}(9)-\mathrm{P}(1)-\mathrm{Pd}(1)$ & $111.81(17)$ \\
\hline $\mathrm{C}(3)-\mathrm{P}(1)-\mathrm{Pd}(1)$ & $115.34(17)$ \\
\hline $\mathrm{C}(1)-\mathrm{P}(1)-\mathrm{Pd}(1)$ & $110.07(16)$ \\
\hline$C(2)-P(2)-C(15)$ & $111.0(2)$ \\
\hline $\mathrm{C}(2)-\mathrm{P}(2)-\mathrm{Pd}(1)$ & $114.87(18)$ \\
\hline $\mathrm{C}(15)-\mathrm{P}(2)-\mathrm{Pd}(1)$ & $133.96(18)$ \\
\hline $\mathrm{Cl}(3) \# 1-\mathrm{Cl}(3)-\mathrm{C}(33)$ & $157.8(7)$ \\
\hline$C(2)-C(1)-P(1)$ & $110.8(4)$ \\
\hline $\mathrm{C}(1)-\mathrm{C}(2)-\mathrm{P}(2)$ & 119.1(4) \\
\hline $\mathrm{C}(8)-\mathrm{C}(3)-\mathrm{C}(4)$ & 111.7(4) \\
\hline $\mathrm{C}(8)-\mathrm{C}(3)-\mathrm{P}(1)$ & $113.7(3)$ \\
\hline $\mathrm{C}(4)-\mathrm{C}(3)-\mathrm{P}(1)$ & 109.7(3) \\
\hline $\mathrm{C}(5)-\mathrm{C}(4)-\mathrm{C}(3)$ & 111.1(4) \\
\hline
\end{tabular}




\begin{tabular}{|c|c|}
\hline$C(6)-C(5)-C(4)$ & $111.6(5)$ \\
\hline$C(7)-C(6)-C(5)$ & $110.5(5)$ \\
\hline$C(6)-C(7)-C(8)$ & $111.4(4)$ \\
\hline $\mathrm{C}(3)-\mathrm{C}(8)-\mathrm{C}(7)$ & $110.7(4)$ \\
\hline $\mathrm{C}(14)-\mathrm{C}(9)-\mathrm{C}(10)$ & $110.9(4)$ \\
\hline $\mathrm{C}(14)-\mathrm{C}(9)-\mathrm{P}(1)$ & $109.7(3)$ \\
\hline $\mathrm{C}(10)-\mathrm{C}(9)-\mathrm{P}(1)$ & $113.3(4)$ \\
\hline $\mathrm{C}(11)-\mathrm{C}(10)-\mathrm{C}(9)$ & $110.7(5)$ \\
\hline$C(12)-C(11)-C(10)$ & $110.9(5)$ \\
\hline$C(13)-C(12)-C(11)$ & $109.9(5)$ \\
\hline$C(12)-C(13)-C(14)$ & $110.7(5)$ \\
\hline $\mathrm{C}(13)-\mathrm{C}(14)-\mathrm{C}(9)$ & 111.1(4) \\
\hline$C(20)-C(15)-C(16)$ & $120.8(4)$ \\
\hline $\mathrm{C}(20)-\mathrm{C}(15)-\mathrm{P}(2)$ & $119.0(4)$ \\
\hline $\mathrm{C}(16)-\mathrm{C}(15)-\mathrm{P}(2)$ & $119.8(4)$ \\
\hline$C(17)-C(16)-C(15)$ & $116.7(5)$ \\
\hline$C(17)-C(16)-C(24)$ & $118.2(4)$ \\
\hline$C(15)-C(16)-C(24)$ & $125.2(5)$ \\
\hline$C(16)-C(17)-C(18)$ & $124.3(5)$ \\
\hline $\mathrm{C}(19)-\mathrm{C}(18)-\mathrm{C}(17)$ & $117.0(5)$ \\
\hline $\mathrm{C}(19)-\mathrm{C}(18)-\mathrm{C}(28)$ & $122.7(5)$ \\
\hline $\mathrm{C}(17)-\mathrm{C}(18)-\mathrm{C}(28)$ & $120.3(5)$ \\
\hline $\mathrm{C}(18)-\mathrm{C}(19)-\mathrm{C}(20)$ & $123.6(5)$ \\
\hline$C(19)-C(20)-C(15)$ & $117.4(5)$ \\
\hline $\mathrm{C}(19)-\mathrm{C}(20)-\mathrm{C}(32)$ & $115.4(5)$ \\
\hline $\mathrm{C}(15)-\mathrm{C}(20)-\mathrm{C}(32)$ & 127.1(4) \\
\hline $\mathrm{C}(23)-\mathrm{C}(24)-\mathrm{C}(21)$ & $106.4(5)$ \\
\hline $\mathrm{C}(23)-\mathrm{C}(24)-\mathrm{C}(22)$ & $105.4(5)$ \\
\hline $\mathrm{C}(21)-\mathrm{C}(24)-\mathrm{C}(22)$ & $110.6(5)$ \\
\hline$C(23)-C(24)-C(16)$ & $111.6(5)$ \\
\hline $\mathrm{C}(21)-\mathrm{C}(24)-\mathrm{C}(16)$ & $110.8(5)$ \\
\hline$C(22)-C(24)-C(16)$ & $111.8(5)$ \\
\hline $\mathrm{C}(25)-\mathrm{C}(28)-\mathrm{C}(18)$ & $112.8(5)$ \\
\hline $\mathrm{C}(25)-\mathrm{C}(28)-\mathrm{C}(27)$ & $107.4(5)$ \\
\hline $\mathrm{C}(18)-\mathrm{C}(28)-\mathrm{C}(27)$ & $110.1(5)$ \\
\hline$C(25)-C(28)-C(26)$ & $109.0(6)$ \\
\hline
\end{tabular}




$\begin{array}{ll}\mathrm{C}(18)-\mathrm{C}(28)-\mathrm{C}(26) & 109.0(5) \\ \mathrm{C}(27)-\mathrm{C}(28)-\mathrm{C}(26) & 108.4(5) \\ \mathrm{C}(31)-\mathrm{C}(32)-\mathrm{C}(29) & 106.1(7) \\ \mathrm{C}(31)-\mathrm{C}(32)-\mathrm{C}(30) & 107.4(6) \\ \mathrm{C}(29)-\mathrm{C}(32)-\mathrm{C}(30) & 107.6(9) \\ \mathrm{C}(31)-\mathrm{C}(32)-\mathrm{C}(20) & 117.5(5) \\ \mathrm{C}(29)-\mathrm{C}(32)-\mathrm{C}(20) & 110.6(5) \\ \mathrm{C}(30)-\mathrm{C}(32)-\mathrm{C}(20) & 107.2(5) \\ \mathrm{Cl}(4)-\mathrm{C}(33)-\mathrm{Cl}(5) & 109.6(3) \\ \mathrm{Cl}(4)-\mathrm{C}(33)-\mathrm{Cl}(3) & 113.0(4) \\ \mathrm{Cl}(5)-\mathrm{C}(33)-\mathrm{Cl}(3) & 109.0(5) \\ \mathrm{Cl}(8)-\mathrm{C}(34)-\mathrm{Cl}(6) & 110.4(3) \\ \mathrm{Cl}(8)-\mathrm{C}(34)-\mathrm{Cl}(7) & 112.3(3) \\ \mathrm{Cl}(6)-\mathrm{C}(34)-\mathrm{Cl}(7) & 110.0(3)\end{array}$

Symmetry transformations used to generate equivalent atoms:

$\# 1-\mathrm{x}+2,-\mathrm{y}+1,-\mathrm{z}+1$ 
Table S15. Anisotropic displacement parameters $\left(\AA^{2} \times 10^{3}\right)$ for CAIN03. The anisotropic displacement factor exponent takes the form: $-2 \pi^{2}\left[h^{2} a^{* 2} U^{11}+\ldots+2 h \mathrm{k}^{*} b^{*} U^{12}\right]$

\begin{tabular}{|c|c|c|c|c|c|c|}
\hline & $\mathrm{U}^{11}$ & $\mathrm{U}^{22}$ & $\mathrm{U}^{33}$ & $\mathrm{U}^{23}$ & $\mathrm{U}^{13}$ & $\mathrm{U}^{12}$ \\
\hline $\operatorname{Pd}(1)$ & 11(1) & $17(1)$ & $19(1)$ & $-1(1)$ & $5(1)$ & $0(1)$ \\
\hline $\mathrm{P}(1)$ & $14(1)$ & $18(1)$ & $20(1)$ & $-2(1)$ & $6(1)$ & $1(1)$ \\
\hline $\mathrm{P}(2)$ & $12(1)$ & 21(1) & $25(1)$ & $0(1)$ & $6(1)$ & $-1(1)$ \\
\hline $\mathrm{Cl}(1)$ & $17(1)$ & $23(1)$ & $38(1)$ & $0(1)$ & $13(1)$ & $-2(1)$ \\
\hline $\mathrm{Cl}(2)$ & $21(1)$ & $22(1)$ & $29(1)$ & $4(1)$ & $6(1)$ & $3(1)$ \\
\hline $\mathrm{Cl}(3)$ & $186(6)$ & $68(3)$ & $108(4)$ & $-34(3)$ & $102(4)$ & $-22(3)$ \\
\hline $\mathrm{Cl}(4)$ & $137(4)$ & $38(2)$ & $33(2)$ & $4(1)$ & $26(2)$ & $29(2)$ \\
\hline $\mathrm{Cl}(5)$ & $211(6)$ & $67(3)$ & $33(2)$ & $-7(2)$ & $-5(3)$ & $87(3)$ \\
\hline $\mathrm{Cl}(6)$ & $114(3)$ & $123(3)$ & $43(2)$ & $10(2)$ & $18(2)$ & $-86(2)$ \\
\hline $\mathrm{Cl}(7)$ & $78(2)$ & $86(2)$ & $62(2)$ & $-23(2)$ & $44(2)$ & $-15(2)$ \\
\hline $\mathrm{Cl}(8)$ & $95(2)$ & $62(2)$ & $76(2)$ & $-19(2)$ & $41(2)$ & $-40(2)$ \\
\hline $\mathrm{C}(1)$ & $19(2)$ & $19(2)$ & $28(3)$ & $4(2)$ & $10(2)$ & $4(2)$ \\
\hline $\mathrm{C}(2)$ & $17(2)$ & $20(2)$ & $23(3)$ & $0(2)$ & $9(2)$ & $-2(2)$ \\
\hline$C(3)$ & $20(2)$ & $16(2)$ & $23(3)$ & $-3(2)$ & $9(2)$ & $3(2)$ \\
\hline$C(4)$ & $19(2)$ & $27(3)$ & $24(3)$ & $-7(2)$ & $5(2)$ & $0(2)$ \\
\hline$C(5)$ & $24(3)$ & $25(3)$ & $26(3)$ & $-8(2)$ & $4(2)$ & $2(2)$ \\
\hline$C(6)$ & $36(3)$ & $29(3)$ & $19(3)$ & $-1(2)$ & $8(2)$ & $9(2)$ \\
\hline$C(7)$ & $28(3)$ & $27(3)$ & $27(3)$ & $-2(2)$ & $10(2)$ & $5(2)$ \\
\hline $\mathrm{C}(8)$ & $18(2)$ & $27(3)$ & $20(3)$ & $-4(2)$ & $10(2)$ & $1(2)$ \\
\hline $\mathrm{C}(9)$ & $16(2)$ & $25(3)$ & $22(3)$ & $1(2)$ & $3(2)$ & $3(2)$ \\
\hline$C(10)$ & $27(3)$ & $30(3)$ & $29(3)$ & $3(3)$ & $7(2)$ & $12(2)$ \\
\hline $\mathrm{C}(11)$ & $24(3)$ & $51(4)$ & $40(4)$ & $10(3)$ & $0(3)$ & $16(3)$ \\
\hline$C(12)$ & $20(3)$ & $51(4)$ & $28(3)$ & $7(3)$ & $-4(2)$ & $4(2)$ \\
\hline$C(13)$ & $24(3)$ & $39(3)$ & $18(3)$ & $-2(2)$ & $0(2)$ & $-2(2)$ \\
\hline$C(14)$ & $17(2)$ & $27(3)$ & $16(3)$ & $1(2)$ & $0(2)$ & $1(2)$ \\
\hline$C(15)$ & $13(2)$ & $25(3)$ & $22(3)$ & $1(2)$ & $6(2)$ & $-1(2)$ \\
\hline$C(16)$ & $19(2)$ & $22(3)$ & $23(3)$ & $-4(2)$ & $2(2)$ & $-4(2)$ \\
\hline $\mathrm{C}(17)$ & $12(2)$ & $30(3)$ & $28(3)$ & $-5(2)$ & $2(2)$ & $-3(2)$ \\
\hline$C(18)$ & $11(2)$ & $25(3)$ & $31(3)$ & $-1(2)$ & $4(2)$ & $2(2)$ \\
\hline $\mathrm{C}(19)$ & $20(3)$ & $30(3)$ & $26(3)$ & $-8(2)$ & $11(2)$ & $4(2)$ \\
\hline $\mathrm{C}(20)$ & $14(2)$ & $28(3)$ & $22(3)$ & $0(2)$ & $7(2)$ & $-1(2)$ \\
\hline
\end{tabular}




\begin{tabular}{lllllll}
$\mathrm{C}(21)$ & $35(3)$ & $59(4)$ & $29(4)$ & $-17(3)$ & $12(3)$ & $-9(3)$ \\
$\mathrm{C}(22)$ & $31(3)$ & $42(4)$ & $47(4)$ & $-22(3)$ & $0(3)$ & $3(3)$ \\
$\mathrm{C}(23)$ & $27(3)$ & $47(4)$ & $29(3)$ & $-14(3)$ & $1(3)$ & $-1(3)$ \\
$\mathrm{C}(24)$ & $18(3)$ & $37(3)$ & $34(3)$ & $-16(3)$ & $6(2)$ & $-7(2)$ \\
$\mathrm{C}(25)$ & $23(3)$ & $67(5)$ & $59(5)$ & $-17(4)$ & $20(3)$ & $-1(3)$ \\
$\mathrm{C}(26)$ & $19(3)$ & $49(4)$ & $78(6)$ & $15(4)$ & $13(3)$ & $5(3)$ \\
$\mathrm{C}(27)$ & $20(3)$ & $40(4)$ & $60(5)$ & $0(3)$ & $11(3)$ & $-6(2)$ \\
$\mathrm{C}(28)$ & $12(2)$ & $37(3)$ & $37(4)$ & $-3(3)$ & $7(2)$ & $0(2)$ \\
$\mathrm{C}(29)$ & $56(5)$ & $128(9)$ & $162(11)$ & $-123(9)$ & $-48(6)$ & $42(6)$ \\
$\mathrm{C}(30)$ & $106(8)$ & $134(9)$ & $30(4)$ & $11(5)$ & $-7(5)$ & $-76(7)$ \\
$\mathrm{C}(31)$ & $36(4)$ & $85(6)$ & $39(4)$ & $-33(4)$ & $16(3)$ & $-35(4)$ \\
$\mathrm{C}(32)$ & $18(3)$ & $48(4)$ & $25(3)$ & $-14(3)$ & $2(2)$ & $3(2)$ \\
$\mathrm{C}(33)$ & $55(7)$ & $46(6)$ & $9(5)$ & $0(4)$ & $20(5)$ & $16(5)$ \\
$\mathrm{C}(34)$ & $36(4)$ & $39(5)$ & $38(5)$ & $-3(4)$ & $9(4)$ & $14(4)$ \\
& & & & & & \\
\hline
\end{tabular}


Table S16. Hydrogen coordinates ( $\left.\mathrm{x} 10^{4}\right)$ and isotropic displacement parameters $\left(\AA^{2} \times 10^{3}\right)$ for CAIN03.

\begin{tabular}{|c|c|c|c|c|}
\hline & $\mathrm{x}$ & $\mathrm{y}$ & $\mathrm{z}$ & $\mathrm{U}(\mathrm{eq})$ \\
\hline $\mathrm{H}(1 \mathrm{~A})$ & 8649 & 951 & 8295 & 25 \\
\hline $\mathrm{H}(1 \mathrm{~B})$ & 8963 & 1651 & 8943 & 25 \\
\hline $\mathrm{H}(2 \mathrm{~A})$ & 7177 & 1661 & 8359 & 23 \\
\hline $\mathrm{H}(3 \mathrm{~A})$ & 9822 & 790 & 7581 & 23 \\
\hline $\mathrm{H}(4 \mathrm{~A})$ & 8186 & 953 & 6941 & 28 \\
\hline $\mathrm{H}(4 \mathrm{~B})$ & 8435 & 1709 & 6443 & 28 \\
\hline $\mathrm{H}(5 \mathrm{~A})$ & 8981 & -116 & 6442 & 31 \\
\hline $\mathrm{H}(5 \mathrm{~B})$ & 8204 & 373 & 5794 & 31 \\
\hline $\mathrm{H}(6 \mathrm{~A})$ & 9698 & 230 & 5528 & 34 \\
\hline $\mathrm{H}(6 \mathrm{~B})$ & 9419 & 1251 & 5557 & 34 \\
\hline $\mathrm{H}(7 \mathrm{~A})$ & 11052 & 1055 & 6213 & 32 \\
\hline $\mathrm{H}(7 \mathrm{~B})$ & 10766 & 314 & 6706 & 32 \\
\hline $\mathrm{H}(8 \mathrm{~A})$ & 11047 & 1656 & 7364 & 25 \\
\hline $\mathrm{H}(8 \mathrm{~B})$ & 10286 & 2163 & 6716 & 25 \\
\hline $\mathrm{H}(9 \mathrm{~A})$ & 11004 & 2699 & 8352 & 26 \\
\hline $\mathrm{H}(10 \mathrm{~A})$ & 10645 & 1293 & 9241 & 34 \\
\hline $\mathrm{H}(10 \mathrm{~B})$ & 11221 & 1189 & 8653 & 34 \\
\hline $\mathrm{H}(11 \mathrm{~A})$ & 12318 & 1318 & 9841 & 48 \\
\hline $\mathrm{H}(11 \mathrm{~B})$ & 12469 & 2113 & 9338 & 48 \\
\hline $\mathrm{H}(12 \mathrm{~A})$ & 11396 & 2254 & 10374 & 42 \\
\hline $\mathrm{H}(12 \mathrm{~B})$ & 12438 & 2683 & 10476 & 42 \\
\hline $\mathrm{H}(13 \mathrm{~A})$ & 11792 & 3630 & 9477 & 34 \\
\hline $\mathrm{H}(13 \mathrm{~B})$ & 11222 & 3773 & 10065 & 34 \\
\hline $\mathrm{H}(14 \mathrm{~A})$ & 10119 & 3654 & 8883 & 25 \\
\hline $\mathrm{H}(14 \mathrm{~B})$ & 9944 & 2873 & 9391 & 25 \\
\hline $\mathrm{H}(17 \mathrm{~A})$ & 3987 & 2926 & 6565 & 29 \\
\hline $\mathrm{H}(19 \mathrm{~A})$ & 4843 & 4112 & 8497 & 30 \\
\hline $\mathrm{H}(21 \mathrm{~A})$ & 6048 & 3583 & 5876 & 60 \\
\hline $\mathrm{H}(21 \mathrm{~B})$ & 6337 & 2691 & 5546 & 60 \\
\hline $\mathrm{H}(21 \mathrm{C})$ & 6928 & 2998 & 6344 & 60 \\
\hline
\end{tabular}




\begin{tabular}{|c|c|c|c|c|}
\hline $\mathrm{H}(22 \mathrm{~A})$ & 6564 & 1505 & 6873 & 64 \\
\hline $\mathrm{H}(22 \mathrm{~B})$ & 6050 & 1216 & 6055 & 64 \\
\hline $\mathrm{H}(22 \mathrm{C})$ & 5475 & 1158 & 6645 & 64 \\
\hline $\mathrm{H}(23 \mathrm{~A})$ & 4376 & 2956 & 5545 & 54 \\
\hline $\mathrm{H}(23 \mathrm{~B})$ & 4181 & 2018 & 5857 & 54 \\
\hline $\mathrm{H}(23 \mathrm{C})$ & 4769 & 2072 & 5275 & 54 \\
\hline $\mathrm{H}(25 \mathrm{~A})$ & 3347 & 3604 & 8597 & 72 \\
\hline $\mathrm{H}(25 \mathrm{~B})$ & 2371 & 4080 & 8148 & 72 \\
\hline $\mathrm{H}(25 \mathrm{C})$ & 3378 & 4590 & 8306 & 72 \\
\hline $\mathrm{H}(26 \mathrm{~A})$ & 2050 & 4381 & 6853 & 74 \\
\hline $\mathrm{H}(26 \mathrm{~B})$ & 2779 & 4051 & 6426 & 74 \\
\hline $\mathrm{H}(26 \mathrm{C})$ & 3066 & 4864 & 6975 & 74 \\
\hline $\mathrm{H}(27 \mathrm{~A})$ & 2929 & 2355 & 7738 & 60 \\
\hline $\mathrm{H}(27 \mathrm{~B})$ & 2703 & 2528 & 6888 & 60 \\
\hline $\mathrm{H}(27 \mathrm{C})$ & 1969 & 2868 & 7308 & 60 \\
\hline $\mathrm{H}(29 \mathrm{~A})$ & 6798 & 5139 & 9527 & 198 \\
\hline H(29B) & 5715 & 4801 & 9186 & 198 \\
\hline $\mathrm{H}(29 \mathrm{C})$ & 6238 & 5350 & 8701 & 198 \\
\hline $\mathrm{H}(30 \mathrm{~A})$ & 7240 & 3599 & 9917 & 143 \\
\hline $\mathrm{H}(30 \mathrm{~B})$ & 7018 & 2821 & 9331 & 143 \\
\hline $\mathrm{H}(30 \mathrm{C})$ & 6152 & 3296 & 9542 & 143 \\
\hline $\mathrm{H}(31 \mathrm{~A})$ & 8090 & 4494 & 9301 & 79 \\
\hline $\mathrm{H}(31 \mathrm{~B})$ & 7680 & 4700 & 8455 & 79 \\
\hline $\mathrm{H}(31 \mathrm{C})$ & 8003 & 3717 & 8723 & 79 \\
\hline $\mathrm{H}(33 \mathrm{~A})$ & 8807 & 4344 & 5935 & 41 \\
\hline $\mathrm{H}(34 \mathrm{~A})$ & 4094 & 4875 & 3410 & 46 \\
\hline
\end{tabular}




\section{Data for 3a}

Table S17. Crystal data and structure refinement for cain15 (3a).

Identification code

Empirical formula

Formula weight

Temperature

cain 15

C34 H57 Cl2 N P2 Pt

807.73

Wavelength

$100.0 \mathrm{~K}$

Crystal system

Space group

Unit cell dimensions

$0.71073 \AA$

Monoclinic

I 1 2/a 1
$\mathrm{a}=17.151(3) \AA$
$\alpha=90^{\circ}$.
$\mathrm{b}=15.554(2) \AA$
$\beta=103.916(3)^{\circ}$.
$\mathrm{c}=28.094(4) \AA$
$\gamma=90^{\circ}$.

Volume

Z

$7274.4(18) \AA^{3}$

Density (calculated)

8

Absorption coefficient

$1.475 \mathrm{Mg} / \mathrm{m}^{3}$

$\mathrm{F}(000)$

$4.116 \mathrm{~mm}^{-1}$

3280

Crystal size

Theta range for data collection

$0.3 \times 0.25 \times 0.2 \mathrm{~mm}^{3}$

1.507 to $28.312^{\circ}$.

Index ranges

Reflections collected

$-22<=\mathrm{h}<=15,-13<=\mathrm{k}<=20,-37<=1<=37$

Independent reflections

Completeness to theta $=26.000^{\circ}$

20113

$9025[\mathrm{R}($ int $)=0.0423]$

Absorption correction

Max. and min. transmission

$99.8 \%$

Semi-empirical from equivalents

0.0962 and 0.0624

Refinement method

Data / restraints / parameters

Full-matrix least-squares on $\mathrm{F}^{2}$

Goodness-of-fit on $\mathrm{F}^{2}$

$9025 / 72$ / 455

Final R indices [I $>2$ sigma(I)]

1.112

$\mathrm{R}$ indices (all data)

$\mathrm{R} 1=0.0553, \mathrm{wR} 2=0.1286$

$\mathrm{R} 1=0.0771, \mathrm{wR} 2=0.1388$

Extinction coefficient

Largest diff. peak and hole

$\mathrm{n} / \mathrm{a}$

4.144 and -1.835 e. $\AA^{-3}$ 
Table S18. Atomic coordinates ( x 104) and equivalent isotropic displacement parameters $\left(\AA^{2} \times 10^{3}\right)$ for cain 15 . $U(e q)$ is defined as one third of the trace of the orthogonalized $U^{i j}$ tensor.

\begin{tabular}{|c|c|c|c|c|}
\hline & $\mathrm{x}$ & $\mathrm{y}$ & $\mathrm{z}$ & $\mathrm{U}(\mathrm{eq})$ \\
\hline $\operatorname{Pt}(01)$ & $5386(1)$ & 7304(1) & $7405(1)$ & $28(1)$ \\
\hline $\mathrm{Cl}(1)$ & $5455(1)$ & $8203(1)$ & $8084(1)$ & $34(1)$ \\
\hline $\mathrm{Cl}(2)$ & $5762(3)$ & $8565(3)$ & $6964(2)$ & $33(1)$ \\
\hline $\mathrm{Cl}(3)$ & $5409(4)$ & $8388(4)$ & $6874(2)$ & $33(1)$ \\
\hline $\mathrm{P}(1)$ & $5212(1)$ & $6138(1)$ & $7836(1)$ & $32(1)$ \\
\hline $\mathrm{P}(2)$ & $5302(1)$ & $6390(1)$ & $6797(1)$ & $30(1)$ \\
\hline $\mathrm{C}(1)$ & $5291(5)$ & $5158(6)$ & $7480(3)$ & $43(2)$ \\
\hline$C(2)$ & $5234(4)$ & $5375(5)$ & $6954(2)$ & $35(2)$ \\
\hline $\mathrm{C}(3)$ & $5964(5)$ & $6040(5)$ & $8426(2)$ & $34(2)$ \\
\hline$C(4)$ & $5807(6)$ & $5307(6)$ & $8742(3)$ & $48(2)$ \\
\hline$C(5)$ & $6450(6)$ & $5286(6)$ & $9232(3)$ & $54(3)$ \\
\hline$C(6)$ & $7273(7)$ & $5238(6)$ & $9148(3)$ & $60(3)$ \\
\hline$C(7)$ & $7439(6)$ & $5966(7)$ & $8827(3)$ & $59(3)$ \\
\hline$C(8)$ & $6804(5)$ & $6005(6)$ & $8343(3)$ & $47(2)$ \\
\hline$C(9)$ & $4304(13)$ & $5896(18)$ & $8000(9)$ & $36(3)$ \\
\hline$C(10)$ & $3603(10)$ & $5826(16)$ & $7557(6)$ & $35(4)$ \\
\hline$C(11)$ & $2824(10)$ & $5607(16)$ & $7709(7)$ & $41(5)$ \\
\hline$C(12)$ & $2646(11)$ & $6274(17)$ & $8075(7)$ & 41(4) \\
\hline$C(13)$ & $3332(13)$ & $6315(16)$ & $8517(8)$ & $36(4)$ \\
\hline$C(14)$ & $4107(17)$ & $6551(19)$ & $8356(11)$ & $35(5)$ \\
\hline$C(15)$ & $4158(13)$ & $6138(18)$ & 7934(9) & $36(3)$ \\
\hline$C(16)$ & $4017(17)$ & $6790(20)$ & $8336(11)$ & $39(5)$ \\
\hline$C(17)$ & $3201(13)$ & $6621(18)$ & $8426(8)$ & $44(5)$ \\
\hline $\mathrm{C}(18)$ & $2513(11)$ & $6736(17)$ & $7960(7)$ & $44(4)$ \\
\hline$C(19)$ & $2674(9)$ & $6133(16)$ & $7558(6)$ & $40(4)$ \\
\hline$C(20)$ & $3495(10)$ & $6258(14)$ & $7456(6)$ & $33(4)$ \\
\hline$C(21)$ & $5292(4)$ & $6536(5)$ & $6151(2)$ & $27(1)$ \\
\hline$C(22)$ & $4570(4)$ & $6734(5)$ & $5803(2)$ & $28(2)$ \\
\hline$C(23)$ & $4582(4)$ & $6722(4)$ & $5308(2)$ & $24(1)$ \\
\hline$C(24)$ & $5261(4)$ & $6539(4)$ & $5145(2)$ & $24(1)$ \\
\hline$C(25)$ & $5959(4)$ & $6386(4)$ & $5500(2)$ & $25(1)$ \\
\hline
\end{tabular}




\begin{tabular}{lcccc}
$\mathrm{C}(26)$ & $6020(4)$ & $6390(4)$ & $6005(2)$ & $24(1)$ \\
$\mathrm{C}(27)$ & $5260(4)$ & $6513(5)$ & $4597(2)$ & $30(2)$ \\
$\mathrm{C}(28)$ & $4429(5)$ & $6637(6)$ & $4267(2)$ & $41(2)$ \\
$\mathrm{C}(29)$ & $5598(5)$ & $5642(5)$ & $4476(3)$ & $40(2)$ \\
$\mathrm{C}(30)$ & $5818(5)$ & $7233(5)$ & $4491(3)$ & $36(2)$ \\
$\mathrm{C}(31)$ & $6861(4)$ & $6216(5)$ & $6344(2)$ & $28(1)$ \\
$\mathrm{C}(32)$ & $6951(5)$ & $5261(6)$ & $6488(3)$ & $47(2)$ \\
$\mathrm{C}(33)$ & $7066(5)$ & $6781(6)$ & $6804(3)$ & $47(2)$ \\
$\mathrm{C}(34)$ & $7524(5)$ & $6396(6)$ & $6064(3)$ & $45(2)$ \\
$\mathrm{C}(35)$ & $3730(4)$ & $6940(6)$ & $5896(3)$ & $41(2)$ \\
$\mathrm{C}(36)$ & $3341(10)$ & $6246(11)$ & $6001(8)$ & $51(5)$ \\
$\mathrm{C}(37)$ & $3092(9)$ & $6159(10)$ & $5587(6)$ & $33(4)$ \\
$\mathrm{C}(38)$ & $3222(11)$ & $7514(14)$ & $5451(7)$ & $46(5)$ \\
$\mathrm{C}(39)$ & $3432(11)$ & $7754(12)$ & $5712(9)$ & $42(5)$ \\
$\mathrm{C}(40)$ & $3860(9)$ & $7691(12)$ & $6336(6)$ & $47(5)$ \\
$\mathrm{C}(41)$ & $3581(9)$ & $6749(12)$ & $6421(5)$ & $38(5)$ \\
$\mathrm{N}(1)$ & $10649(6)$ & $5992(6)$ & $10272(4)$ & $69(3)$ \\
$\mathrm{C}(42)$ & $9551(5)$ & $6153(6)$ & $9457(3)$ & $48(2)$ \\
$\mathrm{C}(43)$ & $10165(6)$ & $6059(6)$ & $9912(4)$ & $49(2)$ \\
& & & & \\
\hline
\end{tabular}


Table S19. Bond lengths $[\AA]$ and angles $\left[{ }^{\circ}\right]$ for cain 15 .

\begin{tabular}{|c|c|}
\hline $\operatorname{Pt}(01)-\mathrm{Cl}(1)$ & $2.3450(17)$ \\
\hline $\mathrm{Pt}(01)-\mathrm{Cl}(2)$ & $2.486(4)$ \\
\hline $\mathrm{Pt}(01)-\mathrm{Cl}(3)$ & $2.257(6)$ \\
\hline$P t(01)-P(1)$ & $2.241(2)$ \\
\hline $\mathrm{Pt}(01)-\mathrm{P}(2)$ & $2.1998(18)$ \\
\hline $\mathrm{P}(1)-\mathrm{C}(1)$ & $1.847(8)$ \\
\hline $\mathrm{P}(1)-\mathrm{C}(3)$ & $1.845(7)$ \\
\hline $\mathrm{P}(1)-\mathrm{C}(9)$ & $1.77(2)$ \\
\hline $\mathrm{P}(1)-\mathrm{C}(15)$ & $1.89(2)$ \\
\hline$P(2)-C(2)$ & $1.651(8)$ \\
\hline$P(2)-C(21)$ & $1.825(7)$ \\
\hline $\mathrm{C}(1)-\mathrm{H}(1 \mathrm{~A})$ & 0.9900 \\
\hline $\mathrm{C}(1)-\mathrm{H}(1 \mathrm{~B})$ & 0.9900 \\
\hline$C(1)-C(2)$ & $1.496(10)$ \\
\hline $\mathrm{C}(2)-\mathrm{H}(2)$ & 0.9500 \\
\hline $\mathrm{C}(3)-\mathrm{H}(3)$ & 1.0000 \\
\hline$C(3)-C(4)$ & $1.509(10)$ \\
\hline$C(3)-C(8)$ & $1.514(12)$ \\
\hline $\mathrm{C}(4)-\mathrm{H}(4 \mathrm{~A})$ & 0.9900 \\
\hline $\mathrm{C}(4)-\mathrm{H}(4 \mathrm{~B})$ & 0.9900 \\
\hline$C(4)-C(5)$ & $1.543(13)$ \\
\hline $\mathrm{C}(5)-\mathrm{H}(5 \mathrm{~A})$ & 0.9900 \\
\hline $\mathrm{C}(5)-\mathrm{H}(5 \mathrm{~B})$ & 0.9900 \\
\hline$C(5)-C(6)$ & $1.487(14)$ \\
\hline $\mathrm{C}(6)-\mathrm{H}(6 \mathrm{~A})$ & 0.9900 \\
\hline $\mathrm{C}(6)-\mathrm{H}(6 \mathrm{~B})$ & 0.9900 \\
\hline$C(6)-C(7)$ & $1.516(13)$ \\
\hline $\mathrm{C}(7)-\mathrm{H}(7 \mathrm{~A})$ & 0.9900 \\
\hline $\mathrm{C}(7)-\mathrm{H}(7 \mathrm{~B})$ & 0.9900 \\
\hline$C(7)-C(8)$ & $1.525(11)$ \\
\hline $\mathrm{C}(8)-\mathrm{H}(8 \mathrm{~A})$ & 0.9900 \\
\hline $\mathrm{C}(8)-\mathrm{H}(8 \mathrm{~B})$ & 0.9900 \\
\hline $\mathrm{C}(9)-\mathrm{H}(9)$ & 1.0000 \\
\hline $\mathrm{C}(9)-\mathrm{C}(10)$ & $1.51(3)$ \\
\hline
\end{tabular}




\begin{tabular}{|c|c|}
\hline$C(9)-C(14)$ & $1.52(3)$ \\
\hline $\mathrm{C}(10)-\mathrm{H}(10 \mathrm{~A})$ & 0.9900 \\
\hline $\mathrm{C}(10)-\mathrm{H}(10 \mathrm{~B})$ & 0.9900 \\
\hline$C(10)-C(11)$ & $1.54(2)$ \\
\hline $\mathrm{C}(11)-\mathrm{H}(11 \mathrm{~A})$ & 0.9900 \\
\hline $\mathrm{C}(11)-\mathrm{H}(11 \mathrm{~B})$ & 0.9900 \\
\hline$C(11)-C(12)$ & $1.54(2)$ \\
\hline $\mathrm{C}(12)-\mathrm{H}(12 \mathrm{~A})$ & 0.9900 \\
\hline $\mathrm{C}(12)-\mathrm{H}(12 \mathrm{~B})$ & 0.9900 \\
\hline$C(12)-C(13)$ & $1.49(2)$ \\
\hline $\mathrm{C}(13)-\mathrm{H}(13 \mathrm{~A})$ & 0.9900 \\
\hline C(13)-H(13B) & 0.9900 \\
\hline$C(13)-C(14)$ & $1.55(3)$ \\
\hline $\mathrm{C}(14)-\mathrm{H}(14 \mathrm{~A})$ & 0.9900 \\
\hline $\mathrm{C}(14)-\mathrm{H}(14 \mathrm{~B})$ & 0.9900 \\
\hline $\mathrm{C}(15)-\mathrm{H}(15)$ & 1.0000 \\
\hline$C(15)-C(16)$ & $1.57(3)$ \\
\hline$C(15)-C(20)$ & $1.55(2)$ \\
\hline $\mathrm{C}(16)-\mathrm{H}(16 \mathrm{~A})$ & 0.9900 \\
\hline $\mathrm{C}(16)-\mathrm{H}(16 \mathrm{~B})$ & 0.9900 \\
\hline$C(16)-C(17)$ & $1.50(3)$ \\
\hline $\mathrm{C}(17)-\mathrm{H}(17 \mathrm{~A})$ & 0.9900 \\
\hline C(17)-H(17B) & 0.9900 \\
\hline $\mathrm{C}(17)-\mathrm{C}(18)$ & $1.55(3)$ \\
\hline $\mathrm{C}(18)-\mathrm{H}(18 \mathrm{~A})$ & 0.9900 \\
\hline C(18)-H(18B) & 0.9900 \\
\hline$C(18)-C(19)$ & $1.54(2)$ \\
\hline C(19)-H(19A) & 0.9900 \\
\hline C(19)-H(19B) & 0.9900 \\
\hline C(19)-C(20) & $1.52(2)$ \\
\hline $\mathrm{C}(20)-\mathrm{H}(20 \mathrm{~A})$ & 0.9900 \\
\hline C(20)-H(20B) & 0.9900 \\
\hline$C(21)-C(22)$ & $1.415(9)$ \\
\hline$C(21)-C(26)$ & $1.423(9)$ \\
\hline$C(22)-C(23)$ & $1.397(9)$ \\
\hline$C(22)-C(35)$ & $1.559(9)$ \\
\hline
\end{tabular}




\begin{tabular}{|c|c|}
\hline $\mathrm{C}(23)-\mathrm{H}(23)$ & 0.9500 \\
\hline$C(23)-C(24)$ & $1.378(9)$ \\
\hline$C(24)-C(25)$ & $1.381(9)$ \\
\hline$C(24)-C(27)$ & $1.541(9)$ \\
\hline $\mathrm{C}(25)-\mathrm{H}(25)$ & 0.9500 \\
\hline$C(25)-C(26)$ & $1.398(9)$ \\
\hline$C(26)-C(31)$ & $1.548(9)$ \\
\hline$C(27)-C(28)$ & $1.514(10)$ \\
\hline$C(27)-C(29)$ & $1.543(10)$ \\
\hline$C(27)-C(30)$ & $1.548(10)$ \\
\hline $\mathrm{C}(28)-\mathrm{H}(28 \mathrm{~A})$ & 0.9800 \\
\hline C(28)-H(28B) & 0.9800 \\
\hline $\mathrm{C}(28)-\mathrm{H}(28 \mathrm{C})$ & 0.9800 \\
\hline C(29)-H(29A) & 0.9800 \\
\hline C(29)-H(29B) & 0.9800 \\
\hline C(29)-H(29C) & 0.9800 \\
\hline $\mathrm{C}(30)-\mathrm{H}(30 \mathrm{~A})$ & 0.9800 \\
\hline $\mathrm{C}(30)-\mathrm{H}(30 \mathrm{~B})$ & 0.9800 \\
\hline $\mathrm{C}(30)-\mathrm{H}(30 \mathrm{C})$ & 0.9800 \\
\hline$C(31)-C(32)$ & $1.538(11)$ \\
\hline$C(31)-C(33)$ & $1.533(10)$ \\
\hline$C(31)-C(34)$ & $1.554(10)$ \\
\hline $\mathrm{C}(32)-\mathrm{H}(32 \mathrm{~A})$ & 0.9800 \\
\hline $\mathrm{C}(32)-\mathrm{H}(32 \mathrm{~B})$ & 0.9800 \\
\hline $\mathrm{C}(32)-\mathrm{H}(32 \mathrm{C})$ & 0.9800 \\
\hline $\mathrm{C}(33)-\mathrm{H}(33 \mathrm{~A})$ & 0.9800 \\
\hline C(33)-H(33B) & 0.9800 \\
\hline C(33)-H(33C) & 0.9800 \\
\hline $\mathrm{C}(34)-\mathrm{H}(34 \mathrm{~A})$ & 0.9800 \\
\hline $\mathrm{C}(34)-\mathrm{H}(34 \mathrm{~B})$ & 0.9800 \\
\hline $\mathrm{C}(34)-\mathrm{H}(34 \mathrm{C})$ & 0.9800 \\
\hline$C(35)-C(36)$ & $1.338(18)$ \\
\hline C(35)-C(37) & $1.724(18)$ \\
\hline C(35)-C(38) & $1.611(19)$ \\
\hline C(35)-C(39) & $1.42(2)$ \\
\hline$C(35)-C(40)$ & $1.675(17)$ \\
\hline
\end{tabular}




\begin{tabular}{|c|c|}
\hline$C(35)-C(41)$ & $1.584(16)$ \\
\hline $\mathrm{C}(36)-\mathrm{H}(36 \mathrm{~A})$ & 0.9800 \\
\hline $\mathrm{C}(36)-\mathrm{H}(36 \mathrm{~B})$ & 0.9800 \\
\hline $\mathrm{C}(36)-\mathrm{H}(36 \mathrm{C})$ & 0.9800 \\
\hline $\mathrm{C}(37)-\mathrm{H}(37 \mathrm{~A})$ & 0.9800 \\
\hline $\mathrm{C}(37)-\mathrm{H}(37 \mathrm{~B})$ & 0.9800 \\
\hline $\mathrm{C}(37)-\mathrm{H}(37 \mathrm{C})$ & 0.9800 \\
\hline $\mathrm{C}(38)-\mathrm{H}(38 \mathrm{~A})$ & 0.9800 \\
\hline $\mathrm{C}(38)-\mathrm{H}(38 \mathrm{~B})$ & 0.9800 \\
\hline $\mathrm{C}(38)-\mathrm{H}(38 \mathrm{C})$ & 0.9800 \\
\hline $\mathrm{C}(39)-\mathrm{H}(39 \mathrm{~A})$ & 0.9800 \\
\hline $\mathrm{C}(39)-\mathrm{H}(39 \mathrm{~B})$ & 0.9800 \\
\hline $\mathrm{C}(39)-\mathrm{H}(39 \mathrm{C})$ & 0.9800 \\
\hline $\mathrm{C}(40)-\mathrm{H}(40 \mathrm{~A})$ & 0.9800 \\
\hline $\mathrm{C}(40)-\mathrm{H}(40 \mathrm{~B})$ & 0.9800 \\
\hline $\mathrm{C}(40)-\mathrm{H}(40 \mathrm{C})$ & 0.9800 \\
\hline $\mathrm{C}(41)-\mathrm{H}(41 \mathrm{~A})$ & 0.9800 \\
\hline $\mathrm{C}(41)-\mathrm{H}(41 \mathrm{~B})$ & 0.9800 \\
\hline $\mathrm{C}(41)-\mathrm{H}(41 \mathrm{C})$ & 0.9800 \\
\hline $\mathrm{N}(1)-\mathrm{C}(43)$ & $1.147(12)$ \\
\hline $\mathrm{C}(42)-\mathrm{H}(42 \mathrm{~A})$ & 0.9800 \\
\hline $\mathrm{C}(42)-\mathrm{H}(42 \mathrm{~B})$ & 0.9800 \\
\hline $\mathrm{C}(42)-\mathrm{H}(42 \mathrm{C})$ & 0.9800 \\
\hline$C(42)-C(43)$ & $1.455(12)$ \\
\hline $\mathrm{Cl}(1)-\mathrm{Pt}(01)-\mathrm{Cl}(2)$ & $88.05(11)$ \\
\hline $\mathrm{Cl}(3)-\mathrm{Pt}(01)-\mathrm{Cl}(1)$ & $94.96(16)$ \\
\hline $\mathrm{P}(1)-\mathrm{Pt}(01)-\mathrm{Cl}(1)$ & $91.73(7)$ \\
\hline $\mathrm{P}(1)-\mathrm{Pt}(01)-\mathrm{Cl}(2)$ & 172.86(13) \\
\hline $\mathrm{P}(1)-\mathrm{Pt}(01)-\mathrm{Cl}(3)$ & 170.91(17) \\
\hline $\mathrm{P}(2)-\mathrm{Pt}(01)-\mathrm{Cl}(1)$ & $176.20(7)$ \\
\hline $\mathrm{P}(2)-\mathrm{Pt}(01)-\mathrm{Cl}(2)$ & $95.74(12)$ \\
\hline $\mathrm{P}(2)-\mathrm{Pt}(01)-\mathrm{Cl}(3)$ & $88.75(16)$ \\
\hline $\mathrm{P}(2)-\mathrm{Pt}(01)-\mathrm{P}(1)$ & $84.50(7)$ \\
\hline $\mathrm{C}(1)-\mathrm{P}(1)-\mathrm{Pt}(01)$ & $109.8(2)$ \\
\hline $\mathrm{C}(1)-\mathrm{P}(1)-\mathrm{C}(15)$ & 106.1(9) \\
\hline
\end{tabular}




\begin{tabular}{|c|c|}
\hline $\mathrm{C}(3)-\mathrm{P}(1)-\mathrm{Pt}(01)$ & $113.4(2)$ \\
\hline $\mathrm{C}(3)-\mathrm{P}(1)-\mathrm{C}(1)$ & $107.0(4)$ \\
\hline $\mathrm{C}(3)-\mathrm{P}(1)-\mathrm{C}(15)$ & $110.8(8)$ \\
\hline $\mathrm{C}(9)-\mathrm{P}(1)-\mathrm{Pt}(01)$ & $123.6(9)$ \\
\hline $\mathrm{C}(9)-\mathrm{P}(1)-\mathrm{C}(1)$ & $98.6(9)$ \\
\hline $\mathrm{C}(9)-\mathrm{P}(1)-\mathrm{C}(3)$ & $102.6(8)$ \\
\hline $\mathrm{C}(15)-\mathrm{P}(1)-\mathrm{Pt}(01)$ & $109.5(9)$ \\
\hline $\mathrm{C}(2)-\mathrm{P}(2)-\mathrm{Pt}(01)$ & $114.0(2)$ \\
\hline $\mathrm{C}(2)-\mathrm{P}(2)-\mathrm{C}(21)$ & $113.6(3)$ \\
\hline $\mathrm{C}(21)-\mathrm{P}(2)-\mathrm{Pt}(01)$ & $132.4(3)$ \\
\hline $\mathrm{P}(1)-\mathrm{C}(1)-\mathrm{H}(1 \mathrm{~A})$ & 109.5 \\
\hline $\mathrm{P}(1)-\mathrm{C}(1)-\mathrm{H}(1 \mathrm{~B})$ & 109.5 \\
\hline $\mathrm{H}(1 \mathrm{~A})-\mathrm{C}(1)-\mathrm{H}(1 \mathrm{~B})$ & 108.1 \\
\hline $\mathrm{C}(2)-\mathrm{C}(1)-\mathrm{P}(1)$ & $110.7(6)$ \\
\hline $\mathrm{C}(2)-\mathrm{C}(1)-\mathrm{H}(1 \mathrm{~A})$ & 109.5 \\
\hline $\mathrm{C}(2)-\mathrm{C}(1)-\mathrm{H}(1 \mathrm{~B})$ & 109.5 \\
\hline $\mathrm{P}(2)-\mathrm{C}(2)-\mathrm{H}(2)$ & 120.4 \\
\hline $\mathrm{C}(1)-\mathrm{C}(2)-\mathrm{P}(2)$ & 119.1(6) \\
\hline $\mathrm{C}(1)-\mathrm{C}(2)-\mathrm{H}(2)$ & 120.4 \\
\hline $\mathrm{P}(1)-\mathrm{C}(3)-\mathrm{H}(3)$ & 106.7 \\
\hline $\mathrm{C}(4)-\mathrm{C}(3)-\mathrm{P}(1)$ & $113.9(6)$ \\
\hline $\mathrm{C}(4)-\mathrm{C}(3)-\mathrm{H}(3)$ & 106.7 \\
\hline $\mathrm{C}(4)-\mathrm{C}(3)-\mathrm{C}(8)$ & $112.0(7)$ \\
\hline $\mathrm{C}(8)-\mathrm{C}(3)-\mathrm{P}(1)$ & $110.5(5)$ \\
\hline $\mathrm{C}(8)-\mathrm{C}(3)-\mathrm{H}(3)$ & 106.7 \\
\hline $\mathrm{C}(3)-\mathrm{C}(4)-\mathrm{H}(4 \mathrm{~A})$ & 109.6 \\
\hline $\mathrm{C}(3)-\mathrm{C}(4)-\mathrm{H}(4 \mathrm{~B})$ & 109.6 \\
\hline$C(3)-C(4)-C(5)$ & $110.5(7)$ \\
\hline $\mathrm{H}(4 \mathrm{~A})-\mathrm{C}(4)-\mathrm{H}(4 \mathrm{~B})$ & 108.1 \\
\hline $\mathrm{C}(5)-\mathrm{C}(4)-\mathrm{H}(4 \mathrm{~A})$ & 109.6 \\
\hline $\mathrm{C}(5)-\mathrm{C}(4)-\mathrm{H}(4 \mathrm{~B})$ & 109.6 \\
\hline $\mathrm{C}(4)-\mathrm{C}(5)-\mathrm{H}(5 \mathrm{~A})$ & 109.4 \\
\hline $\mathrm{C}(4)-\mathrm{C}(5)-\mathrm{H}(5 \mathrm{~B})$ & 109.4 \\
\hline $\mathrm{H}(5 \mathrm{~A})-\mathrm{C}(5)-\mathrm{H}(5 \mathrm{~B})$ & 108.0 \\
\hline$C(6)-C(5)-C(4)$ & $111.3(7)$ \\
\hline $\mathrm{C}(6)-\mathrm{C}(5)-\mathrm{H}(5 \mathrm{~A})$ & 109.4 \\
\hline
\end{tabular}




\begin{tabular}{|c|c|}
\hline $\mathrm{C}(6)-\mathrm{C}(5)-\mathrm{H}(5 \mathrm{~B})$ & 109.4 \\
\hline $\mathrm{C}(5)-\mathrm{C}(6)-\mathrm{H}(6 \mathrm{~A})$ & 109.2 \\
\hline $\mathrm{C}(5)-\mathrm{C}(6)-\mathrm{H}(6 \mathrm{~B})$ & 109.2 \\
\hline$C(5)-C(6)-C(7)$ & $112.2(8)$ \\
\hline $\mathrm{H}(6 \mathrm{~A})-\mathrm{C}(6)-\mathrm{H}(6 \mathrm{~B})$ & 107.9 \\
\hline $\mathrm{C}(7)-\mathrm{C}(6)-\mathrm{H}(6 \mathrm{~A})$ & 109.2 \\
\hline $\mathrm{C}(7)-\mathrm{C}(6)-\mathrm{H}(6 \mathrm{~B})$ & 109.2 \\
\hline $\mathrm{C}(6)-\mathrm{C}(7)-\mathrm{H}(7 \mathrm{~A})$ & 109.4 \\
\hline $\mathrm{C}(6)-\mathrm{C}(7)-\mathrm{H}(7 \mathrm{~B})$ & 109.4 \\
\hline$C(6)-C(7)-C(8)$ & $111.3(8)$ \\
\hline $\mathrm{H}(7 \mathrm{~A})-\mathrm{C}(7)-\mathrm{H}(7 \mathrm{~B})$ & 108.0 \\
\hline $\mathrm{C}(8)-\mathrm{C}(7)-\mathrm{H}(7 \mathrm{~A})$ & 109.4 \\
\hline $\mathrm{C}(8)-\mathrm{C}(7)-\mathrm{H}(7 \mathrm{~B})$ & 109.4 \\
\hline$C(3)-C(8)-C(7)$ & $111.5(7)$ \\
\hline $\mathrm{C}(3)-\mathrm{C}(8)-\mathrm{H}(8 \mathrm{~A})$ & 109.3 \\
\hline $\mathrm{C}(3)-\mathrm{C}(8)-\mathrm{H}(8 \mathrm{~B})$ & 109.3 \\
\hline $\mathrm{C}(7)-\mathrm{C}(8)-\mathrm{H}(8 \mathrm{~A})$ & 109.3 \\
\hline $\mathrm{C}(7)-\mathrm{C}(8)-\mathrm{H}(8 \mathrm{~B})$ & 109.3 \\
\hline $\mathrm{H}(8 \mathrm{~A})-\mathrm{C}(8)-\mathrm{H}(8 \mathrm{~B})$ & 108.0 \\
\hline $\mathrm{P}(1)-\mathrm{C}(9)-\mathrm{H}(9)$ & 107.6 \\
\hline $\mathrm{C}(10)-\mathrm{C}(9)-\mathrm{P}(1)$ & $112.2(15)$ \\
\hline $\mathrm{C}(10)-\mathrm{C}(9)-\mathrm{H}(9)$ & 107.6 \\
\hline $\mathrm{C}(10)-\mathrm{C}(9)-\mathrm{C}(14)$ & $109.4(16)$ \\
\hline $\mathrm{C}(14)-\mathrm{C}(9)-\mathrm{P}(1)$ & $112.2(17)$ \\
\hline $\mathrm{C}(14)-\mathrm{C}(9)-\mathrm{H}(9)$ & 107.6 \\
\hline $\mathrm{C}(9)-\mathrm{C}(10)-\mathrm{H}(10 \mathrm{~A})$ & 109.4 \\
\hline $\mathrm{C}(9)-\mathrm{C}(10)-\mathrm{H}(10 \mathrm{~B})$ & 109.4 \\
\hline $\mathrm{C}(9)-\mathrm{C}(10)-\mathrm{C}(11)$ & 111.1(14) \\
\hline $\mathrm{H}(10 \mathrm{~A})-\mathrm{C}(10)-\mathrm{H}(10 \mathrm{~B})$ & 108.0 \\
\hline $\mathrm{C}(11)-\mathrm{C}(10)-\mathrm{H}(10 \mathrm{~A})$ & 109.4 \\
\hline $\mathrm{C}(11)-\mathrm{C}(10)-\mathrm{H}(10 \mathrm{~B})$ & 109.4 \\
\hline $\mathrm{C}(10)-\mathrm{C}(11)-\mathrm{H}(11 \mathrm{~A})$ & 109.4 \\
\hline $\mathrm{C}(10)-\mathrm{C}(11)-\mathrm{H}(11 \mathrm{~B})$ & 109.4 \\
\hline $\mathrm{C}(10)-\mathrm{C}(11)-\mathrm{C}(12)$ & $111.2(14)$ \\
\hline $\mathrm{H}(11 \mathrm{~A})-\mathrm{C}(11)-\mathrm{H}(11 \mathrm{~B})$ & 108.0 \\
\hline $\mathrm{C}(12)-\mathrm{C}(11)-\mathrm{H}(11 \mathrm{~A})$ & 109.4 \\
\hline
\end{tabular}




\begin{tabular}{|c|c|}
\hline $\mathrm{C}(12)-\mathrm{C}(11)-\mathrm{H}(11 \mathrm{~B})$ & 109.4 \\
\hline $\mathrm{C}(11)-\mathrm{C}(12)-\mathrm{H}(12 \mathrm{~A})$ & 109.7 \\
\hline $\mathrm{C}(11)-\mathrm{C}(12)-\mathrm{H}(12 \mathrm{~B})$ & 109.7 \\
\hline $\mathrm{H}(12 \mathrm{~A})-\mathrm{C}(12)-\mathrm{H}(12 \mathrm{~B})$ & 108.2 \\
\hline $\mathrm{C}(13)-\mathrm{C}(12)-\mathrm{C}(11)$ & $109.9(16)$ \\
\hline $\mathrm{C}(13)-\mathrm{C}(12)-\mathrm{H}(12 \mathrm{~A})$ & 109.7 \\
\hline $\mathrm{C}(13)-\mathrm{C}(12)-\mathrm{H}(12 \mathrm{~B})$ & 109.7 \\
\hline $\mathrm{C}(12)-\mathrm{C}(13)-\mathrm{H}(13 \mathrm{~A})$ & 109.8 \\
\hline $\mathrm{C}(12)-\mathrm{C}(13)-\mathrm{H}(13 \mathrm{~B})$ & 109.8 \\
\hline$C(12)-C(13)-C(14)$ & $109.2(16)$ \\
\hline $\mathrm{H}(13 \mathrm{~A})-\mathrm{C}(13)-\mathrm{H}(13 \mathrm{~B})$ & 108.3 \\
\hline $\mathrm{C}(14)-\mathrm{C}(13)-\mathrm{H}(13 \mathrm{~A})$ & 109.8 \\
\hline $\mathrm{C}(14)-\mathrm{C}(13)-\mathrm{H}(13 \mathrm{~B})$ & 109.8 \\
\hline C(9)-C(14)-C(13) & 112.1(19) \\
\hline $\mathrm{C}(9)-\mathrm{C}(14)-\mathrm{H}(14 \mathrm{~A})$ & 109.2 \\
\hline $\mathrm{C}(9)-\mathrm{C}(14)-\mathrm{H}(14 \mathrm{~B})$ & 109.2 \\
\hline $\mathrm{C}(13)-\mathrm{C}(14)-\mathrm{H}(14 \mathrm{~A})$ & 109.2 \\
\hline $\mathrm{C}(13)-\mathrm{C}(14)-\mathrm{H}(14 \mathrm{~B})$ & 109.2 \\
\hline $\mathrm{H}(14 \mathrm{~A})-\mathrm{C}(14)-\mathrm{H}(14 \mathrm{~B})$ & 107.9 \\
\hline $\mathrm{P}(1)-\mathrm{C}(15)-\mathrm{H}(15)$ & 105.9 \\
\hline $\mathrm{C}(16)-\mathrm{C}(15)-\mathrm{P}(1)$ & $114.9(16)$ \\
\hline $\mathrm{C}(16)-\mathrm{C}(15)-\mathrm{H}(15)$ & 105.9 \\
\hline C(20)-C(15)-P(1) & $113.6(14)$ \\
\hline $\mathrm{C}(20)-\mathrm{C}(15)-\mathrm{H}(15)$ & 105.9 \\
\hline$C(20)-C(15)-C(16)$ & $109.8(16)$ \\
\hline $\mathrm{C}(15)-\mathrm{C}(16)-\mathrm{H}(16 \mathrm{~A})$ & 110.0 \\
\hline $\mathrm{C}(15)-\mathrm{C}(16)-\mathrm{H}(16 \mathrm{~B})$ & 110.0 \\
\hline $\mathrm{H}(16 \mathrm{~A})-\mathrm{C}(16)-\mathrm{H}(16 \mathrm{~B})$ & 108.4 \\
\hline$C(17)-C(16)-C(15)$ & $108.6(18)$ \\
\hline $\mathrm{C}(17)-\mathrm{C}(16)-\mathrm{H}(16 \mathrm{~A})$ & 110.0 \\
\hline $\mathrm{C}(17)-\mathrm{C}(16)-\mathrm{H}(16 \mathrm{~B})$ & 110.0 \\
\hline $\mathrm{C}(16)-\mathrm{C}(17)-\mathrm{H}(17 \mathrm{~A})$ & 109.0 \\
\hline C(16)-C(17)-H(17B) & 109.0 \\
\hline$C(16)-C(17)-C(18)$ & $112.9(17)$ \\
\hline $\mathrm{H}(17 \mathrm{~A})-\mathrm{C}(17)-\mathrm{H}(17 \mathrm{~B})$ & 107.8 \\
\hline $\mathrm{C}(18)-\mathrm{C}(17)-\mathrm{H}(17 \mathrm{~A})$ & 109.0 \\
\hline
\end{tabular}




\begin{tabular}{|c|c|}
\hline $\mathrm{C}(18)-\mathrm{C}(17)-\mathrm{H}(17 \mathrm{~B})$ & 109.0 \\
\hline $\mathrm{C}(17)-\mathrm{C}(18)-\mathrm{H}(18 \mathrm{~A})$ & 110.0 \\
\hline C(17)-C(18)-H(18B) & 110.0 \\
\hline $\mathrm{H}(18 \mathrm{~A})-\mathrm{C}(18)-\mathrm{H}(18 \mathrm{~B})$ & 108.4 \\
\hline$C(19)-C(18)-C(17)$ & $108.3(15)$ \\
\hline $\mathrm{C}(19)-\mathrm{C}(18)-\mathrm{H}(18 \mathrm{~A})$ & 110.0 \\
\hline C(19)-C(18)-H(18B) & 110.0 \\
\hline C(18)-C(19)-H(19A) & 108.9 \\
\hline C(18)-C(19)-H(19B) & 108.9 \\
\hline $\mathrm{H}(19 \mathrm{~A})-\mathrm{C}(19)-\mathrm{H}(19 \mathrm{~B})$ & 107.7 \\
\hline$C(20)-C(19)-C(18)$ & $113.5(14)$ \\
\hline C(20)-C(19)-H(19A) & 108.9 \\
\hline C(20)-C(19)-H(19B) & 108.9 \\
\hline$C(15)-C(20)-H(20 A)$ & 109.7 \\
\hline C(15)-C(20)-H(20B) & 109.7 \\
\hline$C(19)-C(20)-C(15)$ & $110.0(14)$ \\
\hline $\mathrm{C}(19)-\mathrm{C}(20)-\mathrm{H}(20 \mathrm{~A})$ & 109.7 \\
\hline C(19)-C(20)-H(20B) & 109.7 \\
\hline $\mathrm{H}(20 \mathrm{~A})-\mathrm{C}(20)-\mathrm{H}(20 \mathrm{~B})$ & 108.2 \\
\hline $\mathrm{C}(22)-\mathrm{C}(21)-\mathrm{P}(2)$ & $120.8(5)$ \\
\hline$C(22)-C(21)-C(26)$ & $121.2(6)$ \\
\hline $\mathrm{C}(26)-\mathrm{C}(21)-\mathrm{P}(2)$ & $117.9(5)$ \\
\hline $\mathrm{C}(21)-\mathrm{C}(22)-\mathrm{C}(35)$ & $128.2(6)$ \\
\hline $\mathrm{C}(23)-\mathrm{C}(22)-\mathrm{C}(21)$ & $117.8(6)$ \\
\hline $\mathrm{C}(23)-\mathrm{C}(22)-\mathrm{C}(35)$ & $114.0(6)$ \\
\hline $\mathrm{C}(22)-\mathrm{C}(23)-\mathrm{H}(23)$ & 118.4 \\
\hline$C(24)-C(23)-C(22)$ & $123.2(6)$ \\
\hline $\mathrm{C}(24)-\mathrm{C}(23)-\mathrm{H}(23)$ & 118.4 \\
\hline$C(23)-C(24)-C(25)$ & $116.9(6)$ \\
\hline$C(23)-C(24)-C(27)$ & $122.6(6)$ \\
\hline$C(25)-C(24)-C(27)$ & $120.6(6)$ \\
\hline $\mathrm{C}(24)-\mathrm{C}(25)-\mathrm{H}(25)$ & 117.5 \\
\hline$C(24)-C(25)-C(26)$ & $124.9(6)$ \\
\hline $\mathrm{C}(26)-\mathrm{C}(25)-\mathrm{H}(25)$ & 117.5 \\
\hline$C(21)-C(26)-C(31)$ & $127.1(6)$ \\
\hline $\mathrm{C}(25)-\mathrm{C}(26)-\mathrm{C}(21)$ & $115.9(6)$ \\
\hline
\end{tabular}




\begin{tabular}{|c|c|}
\hline$C(25)-C(26)-C(31)$ & $117.0(6)$ \\
\hline$C(24)-C(27)-C(29)$ & $109.4(6)$ \\
\hline$C(24)-C(27)-C(30)$ & $108.8(6)$ \\
\hline $\mathrm{C}(28)-\mathrm{C}(27)-\mathrm{C}(24)$ & $112.5(6)$ \\
\hline $\mathrm{C}(28)-\mathrm{C}(27)-\mathrm{C}(29)$ & $109.0(6)$ \\
\hline $\mathrm{C}(28)-\mathrm{C}(27)-\mathrm{C}(30)$ & $108.9(6)$ \\
\hline$C(29)-C(27)-C(30)$ & $108.1(6)$ \\
\hline $\mathrm{C}(27)-\mathrm{C}(28)-\mathrm{H}(28 \mathrm{~A})$ & 109.5 \\
\hline $\mathrm{C}(27)-\mathrm{C}(28)-\mathrm{H}(28 \mathrm{~B})$ & 109.5 \\
\hline $\mathrm{C}(27)-\mathrm{C}(28)-\mathrm{H}(28 \mathrm{C})$ & 109.5 \\
\hline $\mathrm{H}(28 \mathrm{~A})-\mathrm{C}(28)-\mathrm{H}(28 \mathrm{~B})$ & 109.5 \\
\hline $\mathrm{H}(28 \mathrm{~A})-\mathrm{C}(28)-\mathrm{H}(28 \mathrm{C})$ & 109.5 \\
\hline $\mathrm{H}(28 \mathrm{~B})-\mathrm{C}(28)-\mathrm{H}(28 \mathrm{C})$ & 109.5 \\
\hline $\mathrm{C}(27)-\mathrm{C}(29)-\mathrm{H}(29 \mathrm{~A})$ & 109.5 \\
\hline $\mathrm{C}(27)-\mathrm{C}(29)-\mathrm{H}(29 \mathrm{~B})$ & 109.5 \\
\hline $\mathrm{C}(27)-\mathrm{C}(29)-\mathrm{H}(29 \mathrm{C})$ & 109.5 \\
\hline $\mathrm{H}(29 \mathrm{~A})-\mathrm{C}(29)-\mathrm{H}(29 \mathrm{~B})$ & 109.5 \\
\hline $\mathrm{H}(29 \mathrm{~A})-\mathrm{C}(29)-\mathrm{H}(29 \mathrm{C})$ & 109.5 \\
\hline $\mathrm{H}(29 \mathrm{~B})-\mathrm{C}(29)-\mathrm{H}(29 \mathrm{C})$ & 109.5 \\
\hline $\mathrm{C}(27)-\mathrm{C}(30)-\mathrm{H}(30 \mathrm{~A})$ & 109.5 \\
\hline $\mathrm{C}(27)-\mathrm{C}(30)-\mathrm{H}(30 \mathrm{~B})$ & 109.5 \\
\hline $\mathrm{C}(27)-\mathrm{C}(30)-\mathrm{H}(30 \mathrm{C})$ & 109.5 \\
\hline $\mathrm{H}(30 \mathrm{~A})-\mathrm{C}(30)-\mathrm{H}(30 \mathrm{~B})$ & 109.5 \\
\hline $\mathrm{H}(30 \mathrm{~A})-\mathrm{C}(30)-\mathrm{H}(30 \mathrm{C})$ & 109.5 \\
\hline $\mathrm{H}(30 \mathrm{~B})-\mathrm{C}(30)-\mathrm{H}(30 \mathrm{C})$ & 109.5 \\
\hline$C(26)-C(31)-C(34)$ & $110.2(6)$ \\
\hline$C(32)-C(31)-C(26)$ & $110.5(6)$ \\
\hline $\mathrm{C}(32)-\mathrm{C}(31)-\mathrm{C}(34)$ & $105.6(6)$ \\
\hline$C(33)-C(31)-C(26)$ & $114.1(6)$ \\
\hline $\mathrm{C}(33)-\mathrm{C}(31)-\mathrm{C}(32)$ & $110.0(7)$ \\
\hline $\mathrm{C}(33)-\mathrm{C}(31)-\mathrm{C}(34)$ & $106.0(6)$ \\
\hline $\mathrm{C}(31)-\mathrm{C}(32)-\mathrm{H}(32 \mathrm{~A})$ & 109.5 \\
\hline $\mathrm{C}(31)-\mathrm{C}(32)-\mathrm{H}(32 \mathrm{~B})$ & 109.5 \\
\hline $\mathrm{C}(31)-\mathrm{C}(32)-\mathrm{H}(32 \mathrm{C})$ & 109.5 \\
\hline $\mathrm{H}(32 \mathrm{~A})-\mathrm{C}(32)-\mathrm{H}(32 \mathrm{~B})$ & 109.5 \\
\hline $\mathrm{H}(32 \mathrm{~A})-\mathrm{C}(32)-\mathrm{H}(32 \mathrm{C})$ & 109.5 \\
\hline
\end{tabular}




\begin{tabular}{|c|c|}
\hline $\mathrm{H}(32 \mathrm{~B})-\mathrm{C}(32)-\mathrm{H}(32 \mathrm{C})$ & 109.5 \\
\hline $\mathrm{C}(31)-\mathrm{C}(33)-\mathrm{H}(33 \mathrm{~A})$ & 109.5 \\
\hline $\mathrm{C}(31)-\mathrm{C}(33)-\mathrm{H}(33 \mathrm{~B})$ & 109.5 \\
\hline $\mathrm{C}(31)-\mathrm{C}(33)-\mathrm{H}(33 \mathrm{C})$ & 109.5 \\
\hline $\mathrm{H}(33 \mathrm{~A})-\mathrm{C}(33)-\mathrm{H}(33 \mathrm{~B})$ & 109.5 \\
\hline $\mathrm{H}(33 \mathrm{~A})-\mathrm{C}(33)-\mathrm{H}(33 \mathrm{C})$ & 109.5 \\
\hline $\mathrm{H}(33 \mathrm{~B})-\mathrm{C}(33)-\mathrm{H}(33 \mathrm{C})$ & 109.5 \\
\hline $\mathrm{C}(31)-\mathrm{C}(34)-\mathrm{H}(34 \mathrm{~A})$ & 109.5 \\
\hline $\mathrm{C}(31)-\mathrm{C}(34)-\mathrm{H}(34 \mathrm{~B})$ & 109.5 \\
\hline $\mathrm{C}(31)-\mathrm{C}(34)-\mathrm{H}(34 \mathrm{C})$ & 109.5 \\
\hline $\mathrm{H}(34 \mathrm{~A})-\mathrm{C}(34)-\mathrm{H}(34 \mathrm{~B})$ & 109.5 \\
\hline $\mathrm{H}(34 \mathrm{~A})-\mathrm{C}(34)-\mathrm{H}(34 \mathrm{C})$ & 109.5 \\
\hline $\mathrm{H}(34 \mathrm{~B})-\mathrm{C}(34)-\mathrm{H}(34 \mathrm{C})$ & 109.5 \\
\hline$C(22)-C(35)-C(37)$ & $105.7(7)$ \\
\hline$C(22)-C(35)-C(38)$ & $109.4(8)$ \\
\hline$C(22)-C(35)-C(40)$ & $107.6(8)$ \\
\hline$C(22)-C(35)-C(41)$ & $118.7(8)$ \\
\hline$C(36)-C(35)-C(22)$ & $113.7(10)$ \\
\hline $\mathrm{C}(36)-\mathrm{C}(35)-\mathrm{C}(38)$ & $114.3(13)$ \\
\hline$C(36)-C(35)-C(40)$ & $112.7(12)$ \\
\hline $\mathrm{C}(38)-\mathrm{C}(35)-\mathrm{C}(40)$ & $97.9(11)$ \\
\hline $\mathrm{C}(39)-\mathrm{C}(35)-\mathrm{C}(22)$ & $112.9(10)$ \\
\hline $\mathrm{C}(39)-\mathrm{C}(35)-\mathrm{C}(37)$ & $108.4(11)$ \\
\hline$C(39)-C(35)-C(41)$ & $112.6(12)$ \\
\hline $\mathrm{C}(41)-\mathrm{C}(35)-\mathrm{C}(37)$ & $96.4(10)$ \\
\hline $\mathrm{C}(35)-\mathrm{C}(36)-\mathrm{H}(36 \mathrm{~A})$ & 109.5 \\
\hline $\mathrm{C}(35)-\mathrm{C}(36)-\mathrm{H}(36 \mathrm{~B})$ & 109.5 \\
\hline $\mathrm{C}(35)-\mathrm{C}(36)-\mathrm{H}(36 \mathrm{C})$ & 109.5 \\
\hline $\mathrm{H}(36 \mathrm{~A})-\mathrm{C}(36)-\mathrm{H}(36 \mathrm{~B})$ & 109.5 \\
\hline $\mathrm{H}(36 \mathrm{~A})-\mathrm{C}(36)-\mathrm{H}(36 \mathrm{C})$ & 109.5 \\
\hline $\mathrm{H}(36 \mathrm{~B})-\mathrm{C}(36)-\mathrm{H}(36 \mathrm{C})$ & 109.5 \\
\hline $\mathrm{C}(35)-\mathrm{C}(37)-\mathrm{H}(37 \mathrm{~A})$ & 109.5 \\
\hline C(35)-C(37)-H(37B) & 109.5 \\
\hline C(35)-C(37)-H(37C) & 109.5 \\
\hline $\mathrm{H}(37 \mathrm{~A})-\mathrm{C}(37)-\mathrm{H}(37 \mathrm{~B})$ & 109.5 \\
\hline $\mathrm{H}(37 \mathrm{~A})-\mathrm{C}(37)-\mathrm{H}(37 \mathrm{C})$ & 109.5 \\
\hline
\end{tabular}




\begin{tabular}{|c|c|}
\hline $\mathrm{H}(37 \mathrm{~B})-\mathrm{C}(37)-\mathrm{H}(37 \mathrm{C})$ & 109.5 \\
\hline $\mathrm{C}(35)-\mathrm{C}(38)-\mathrm{H}(38 \mathrm{~A})$ & 109.5 \\
\hline $\mathrm{C}(35)-\mathrm{C}(38)-\mathrm{H}(38 \mathrm{~B})$ & 109.5 \\
\hline $\mathrm{C}(35)-\mathrm{C}(38)-\mathrm{H}(38 \mathrm{C})$ & 109.5 \\
\hline $\mathrm{H}(38 \mathrm{~A})-\mathrm{C}(38)-\mathrm{H}(38 \mathrm{~B})$ & 109.5 \\
\hline $\mathrm{H}(38 \mathrm{~A})-\mathrm{C}(38)-\mathrm{H}(38 \mathrm{C})$ & 109.5 \\
\hline $\mathrm{H}(38 \mathrm{~B})-\mathrm{C}(38)-\mathrm{H}(38 \mathrm{C})$ & 109.5 \\
\hline $\mathrm{C}(35)-\mathrm{C}(39)-\mathrm{H}(39 \mathrm{~A})$ & 109.5 \\
\hline $\mathrm{C}(35)-\mathrm{C}(39)-\mathrm{H}(39 \mathrm{~B})$ & 109.5 \\
\hline $\mathrm{C}(35)-\mathrm{C}(39)-\mathrm{H}(39 \mathrm{C})$ & 109.5 \\
\hline H(39A)-C(39)-H(39B) & 109.5 \\
\hline H(39A)-C(39)-H(39C) & 109.5 \\
\hline H(39B)-C(39)-H(39C) & 109.5 \\
\hline $\mathrm{C}(35)-\mathrm{C}(40)-\mathrm{H}(40 \mathrm{~A})$ & 109.5 \\
\hline $\mathrm{C}(35)-\mathrm{C}(40)-\mathrm{H}(40 \mathrm{~B})$ & 109.5 \\
\hline $\mathrm{C}(35)-\mathrm{C}(40)-\mathrm{H}(40 \mathrm{C})$ & 109.5 \\
\hline $\mathrm{H}(40 \mathrm{~A})-\mathrm{C}(40)-\mathrm{H}(40 \mathrm{~B})$ & 109.5 \\
\hline $\mathrm{H}(40 \mathrm{~A})-\mathrm{C}(40)-\mathrm{H}(40 \mathrm{C})$ & 109.5 \\
\hline $\mathrm{H}(40 \mathrm{~B})-\mathrm{C}(40)-\mathrm{H}(40 \mathrm{C})$ & 109.5 \\
\hline $\mathrm{C}(35)-\mathrm{C}(41)-\mathrm{H}(41 \mathrm{~A})$ & 109.5 \\
\hline $\mathrm{C}(35)-\mathrm{C}(41)-\mathrm{H}(41 \mathrm{~B})$ & 109.5 \\
\hline $\mathrm{C}(35)-\mathrm{C}(41)-\mathrm{H}(41 \mathrm{C})$ & 109.5 \\
\hline $\mathrm{H}(41 \mathrm{~A})-\mathrm{C}(41)-\mathrm{H}(41 \mathrm{~B})$ & 109.5 \\
\hline $\mathrm{H}(41 \mathrm{~A})-\mathrm{C}(41)-\mathrm{H}(41 \mathrm{C})$ & 109.5 \\
\hline $\mathrm{H}(41 \mathrm{~B})-\mathrm{C}(41)-\mathrm{H}(41 \mathrm{C})$ & 109.5 \\
\hline $\mathrm{H}(42 \mathrm{~A})-\mathrm{C}(42)-\mathrm{H}(42 \mathrm{~B})$ & 109.5 \\
\hline $\mathrm{H}(42 \mathrm{~A})-\mathrm{C}(42)-\mathrm{H}(42 \mathrm{C})$ & 109.5 \\
\hline $\mathrm{H}(42 \mathrm{~B})-\mathrm{C}(42)-\mathrm{H}(42 \mathrm{C})$ & 109.5 \\
\hline $\mathrm{C}(43)-\mathrm{C}(42)-\mathrm{H}(42 \mathrm{~A})$ & 109.5 \\
\hline $\mathrm{C}(43)-\mathrm{C}(42)-\mathrm{H}(42 \mathrm{~B})$ & 109.5 \\
\hline $\mathrm{C}(43)-\mathrm{C}(42)-\mathrm{H}(42 \mathrm{C})$ & 109.5 \\
\hline $\mathrm{N}(1)-\mathrm{C}(43)-\mathrm{C}(42)$ & $179.4(11)$ \\
\hline
\end{tabular}

Symmetry transformations used to generate equivalent atoms: 
Table S20. Anisotropic displacement parameters $\left(\AA^{2} \times 10^{3}\right)$ for cain15. The anisotropic displacement factor exponent takes the form: $-2 \pi^{2}\left[h^{2} a^{* 2} U^{11}+\ldots+2 h k a^{*} b^{*} U^{12}\right]$

\begin{tabular}{|c|c|c|c|c|c|c|}
\hline & $\mathrm{U}^{11}$ & $\mathrm{U}^{22}$ & $\mathrm{U}^{33}$ & $\mathrm{U}^{23}$ & $\mathrm{U}^{13}$ & $\mathrm{U}^{12}$ \\
\hline $\operatorname{Pt}(01)$ & $32(1)$ & $36(1)$ & $17(1)$ & $0(1)$ & $9(1)$ & $10(1)$ \\
\hline $\mathrm{Cl}(1)$ & $48(1)$ & $33(1)$ & $22(1)$ & $-2(1)$ & $10(1)$ & $6(1)$ \\
\hline $\mathrm{Cl}(2)$ & $52(3)$ & $22(2)$ & $31(2)$ & $4(1)$ & $21(2)$ & $1(2)$ \\
\hline $\mathrm{Cl}(3)$ & $52(3)$ & $22(2)$ & $31(2)$ & $4(1)$ & $21(2)$ & $1(2)$ \\
\hline $\mathrm{P}(1)$ & $38(1)$ & $42(1)$ & 19(1) & $-6(1)$ & $14(1)$ & $-16(1)$ \\
\hline $\mathrm{P}(2)$ & $32(1)$ & $43(1)$ & $17(1)$ & $-3(1)$ & $10(1)$ & $4(1)$ \\
\hline $\mathrm{C}(1)$ & $64(5)$ & $45(5)$ & $25(4)$ & $-13(3)$ & $21(4)$ & $-28(4)$ \\
\hline$C(2)$ & $41(4)$ & $47(5)$ & 21(3) & $-12(3)$ & $17(3)$ & $-15(3)$ \\
\hline$C(3)$ & $52(4)$ & $30(4)$ & 20(3) & $0(3)$ & $9(3)$ & $-12(3)$ \\
\hline$C(4)$ & $80(6)$ & $37(5)$ & $36(4)$ & $5(4)$ & $31(4)$ & $-4(4)$ \\
\hline$C(5)$ & $97(8)$ & $41(5)$ & $32(4)$ & $11(4)$ & $30(5)$ & $17(5)$ \\
\hline$C(6)$ & $88(8)$ & $48(6)$ & $40(5)$ & $9(4)$ & $8(5)$ & $16(5)$ \\
\hline$C(7)$ & $58(6)$ & $63(7)$ & $46(5)$ & $15(5)$ & $-8(4)$ & $-5(5)$ \\
\hline $\mathrm{C}(8)$ & $51(5)$ & $55(6)$ & $31(4)$ & $9(4)$ & $5(4)$ & $-9(4)$ \\
\hline $\mathrm{C}(9)$ & $30(4)$ & $56(10)$ & $25(4)$ & $-9(5)$ & $15(3)$ & $-8(4)$ \\
\hline$C(10)$ & $30(5)$ & $55(11)$ & $25(5)$ & $-9(5)$ & $14(4)$ & $-8(5)$ \\
\hline $\mathrm{C}(11)$ & $32(5)$ & $66(11)$ & $29(6)$ & $-16(7)$ & $16(4)$ & $-13(5)$ \\
\hline$C(12)$ & $33(5)$ & $66(11)$ & $29(6)$ & $-13(6)$ & $16(4)$ & $-9(5)$ \\
\hline$C(13)$ & $34(6)$ & $52(11)$ & $26(5)$ & $-13(6)$ & $17(4)$ & $-9(6)$ \\
\hline $\mathrm{C}(14)$ & $33(6)$ & $54(11)$ & $24(5)$ & $-7(6)$ & $16(4)$ & $-7(6)$ \\
\hline$C(15)$ & $30(4)$ & $56(10)$ & $25(4)$ & $-9(5)$ & $15(3)$ & $-8(4)$ \\
\hline$C(16)$ & $34(6)$ & $61(12)$ & $28(5)$ & $-14(7)$ & $17(4)$ & $-7(6)$ \\
\hline$C(17)$ & $34(6)$ & $70(13)$ & $32(6)$ & $-13(6)$ & $18(4)$ & $-7(6)$ \\
\hline $\mathrm{C}(18)$ & $33(5)$ & $69(11)$ & $34(6)$ & $-15(6)$ & $17(4)$ & $-7(6)$ \\
\hline $\mathrm{C}(19)$ & $32(5)$ & $62(11)$ & $30(6)$ & $-10(6)$ & $16(4)$ & $-7(5)$ \\
\hline$C(20)$ & $30(4)$ & $45(10)$ & $27(5)$ & $-11(5)$ & $13(4)$ & $-6(5)$ \\
\hline $\mathrm{C}(21)$ & $34(4)$ & $31(4)$ & $18(3)$ & $-3(3)$ & $9(3)$ & $5(3)$ \\
\hline$C(22)$ & $33(3)$ & $33(4)$ & 21(3) & $-6(3)$ & $12(3)$ & $4(3)$ \\
\hline $\mathrm{C}(23)$ & $27(3)$ & $25(3)$ & 19(3) & $1(2)$ & $4(2)$ & $8(3)$ \\
\hline$C(24)$ & $39(4)$ & $17(3)$ & $20(3)$ & $0(2)$ & $12(3)$ & $-2(3)$ \\
\hline$C(25)$ & $32(3)$ & $25(3)$ & 23(3) & $-3(3)$ & $15(3)$ & $-1(3)$ \\
\hline
\end{tabular}




\begin{tabular}{|c|c|c|c|c|c|c|}
\hline$C(26)$ & $30(3)$ & $21(3)$ & $23(3)$ & 2(3) & $10(3)$ & $5(3)$ \\
\hline$C(27)$ & $42(4)$ & $28(4)$ & $22(3)$ & 2(3) & $12(3)$ & $-5(3)$ \\
\hline$C(28)$ & $45(4)$ & $62(6)$ & $16(3)$ & 2(3) & $9(3)$ & $-12(4)$ \\
\hline$C(29)$ & $69(5)$ & $27(4)$ & $31(4)$ & $-12(3)$ & $26(4)$ & $-4(4)$ \\
\hline$C(30)$ & $50(4)$ & $37(4)$ & $27(4)$ & $5(3)$ & $19(3)$ & $0(4)$ \\
\hline$C(31)$ & $31(3)$ & $36(4)$ & $21(3)$ & $0(3)$ & $12(3)$ & 1(3) \\
\hline$C(32)$ & $36(4)$ & $51(5)$ & $51(5)$ & $14(4)$ & $7(4)$ & $14(4)$ \\
\hline$C(33)$ & $33(4)$ & $66(6)$ & $42(5)$ & $-18(4)$ & $7(3)$ & $-9(4)$ \\
\hline$C(34)$ & $29(4)$ & $71(6)$ & $35(4)$ & $4(4)$ & $10(3)$ & $4(4)$ \\
\hline$C(35)$ & $33(4)$ & $69(6)$ & $23(3)$ & $-3(4)$ & $8(3)$ & $22(4)$ \\
\hline$C(36)$ & $46(10)$ & $39(10)$ & $79(15)$ & $-6(9)$ & $39(10)$ & $-11(8)$ \\
\hline$C(37)$ & $32(8)$ & $30(8)$ & $36(9)$ & $-2(7)$ & $9(7)$ & $1(6)$ \\
\hline$C(38)$ & $33(9)$ & $74(15)$ & $30(9)$ & $-5(9)$ & $6(7)$ & $16(8)$ \\
\hline$C(39)$ & $32(9)$ & $30(10)$ & $63(14)$ & $10(10)$ & 11(9) & $7(7)$ \\
\hline$C(40)$ & $29(7)$ & $62(11)$ & $48(9)$ & $-28(9)$ & $4(6)$ & $23(7)$ \\
\hline$C(41)$ & $24(7)$ & $66(13)$ & $27(8)$ & $-3(7)$ & $15(6)$ & $4(7)$ \\
\hline $\mathrm{N}(1)$ & $68(6)$ & $51(5)$ & $74(6)$ & $1(5)$ & $-11(5)$ & $1(4)$ \\
\hline$C(42)$ & $50(5)$ & $43(5)$ & $48(5)$ & $1(4)$ & $7(4)$ & $1(4)$ \\
\hline $\mathrm{C}(43)$ & $47(5)$ & $36(5)$ & $62(6)$ & $-2(4)$ & $8(4)$ & $4(4)$ \\
\hline
\end{tabular}


Table S21. Hydrogen coordinates ( x 10 $)$ and isotropic displacement parameters $\left(\AA^{2} \times 10^{3}\right)$ for cain 15 .

\begin{tabular}{|c|c|c|c|c|}
\hline & $\mathrm{x}$ & $\mathrm{y}$ & $\mathrm{z}$ & $\mathrm{U}(\mathrm{eq})$ \\
\hline $\mathrm{H}(1 \mathrm{~A})$ & 5811 & 4870 & 7620 & 51 \\
\hline $\mathrm{H}(1 \mathrm{~B})$ & 4855 & 4754 & 7502 & 51 \\
\hline $\mathrm{H}(2)$ & 5158 & 4934 & 6713 & 42 \\
\hline $\mathrm{H}(3)$ & 5931 & 6581 & 8613 & 41 \\
\hline $\mathrm{H}(4 \mathrm{~A})$ & 5814 & 4757 & 8566 & 58 \\
\hline $\mathrm{H}(4 \mathrm{~B})$ & 5268 & 5376 & 8808 & 58 \\
\hline $\mathrm{H}(5 \mathrm{~A})$ & 6403 & 5810 & 9423 & 65 \\
\hline $\mathrm{H}(5 \mathrm{~B})$ & 6357 & 4782 & 9426 & 65 \\
\hline $\mathrm{H}(6 \mathrm{~A})$ & 7338 & 4682 & 8990 & 72 \\
\hline $\mathrm{H}(6 \mathrm{~B})$ & 7671 & 5259 & 9468 & 72 \\
\hline $\mathrm{H}(7 \mathrm{~A})$ & 7975 & 5883 & 8760 & 71 \\
\hline $\mathrm{H}(7 \mathrm{~B})$ & 7446 & 6518 & 9004 & 71 \\
\hline $\mathrm{H}(8 \mathrm{~A})$ & 6897 & 6520 & 8158 & 56 \\
\hline $\mathrm{H}(8 \mathrm{~B})$ & 6854 & 5492 & 8145 & 56 \\
\hline $\mathrm{H}(9)$ & 4371 & 5325 & 8168 & 43 \\
\hline $\mathrm{H}(10 \mathrm{~A})$ & 3716 & 5373 & 7335 & 42 \\
\hline $\mathrm{H}(10 \mathrm{~B})$ & 3535 & 6377 & 7376 & 42 \\
\hline $\mathrm{H}(11 \mathrm{~A})$ & 2873 & 5030 & 7862 & 49 \\
\hline $\mathrm{H}(11 \mathrm{~B})$ & 2371 & 5593 & 7415 & 49 \\
\hline $\mathrm{H}(12 \mathrm{~A})$ & 2560 & 6846 & 7917 & 49 \\
\hline $\mathrm{H}(12 \mathrm{~B})$ & 2150 & 6110 & 8174 & 49 \\
\hline $\mathrm{H}(13 \mathrm{~A})$ & 3400 & 5753 & 8686 & 43 \\
\hline $\mathrm{H}(13 \mathrm{~B})$ & 3223 & 6754 & 8748 & 43 \\
\hline $\mathrm{H}(14 \mathrm{~A})$ & 4040 & 7124 & 8199 & 43 \\
\hline $\mathrm{H}(14 \mathrm{~B})$ & 4561 & 6584 & 8650 & 43 \\
\hline $\mathrm{H}(15)$ & 4073 & 5552 & 8059 & 43 \\
\hline $\mathrm{H}(16 \mathrm{~A})$ & 4436 & 6707 & 8644 & 47 \\
\hline $\mathrm{H}(16 \mathrm{~B})$ & 4049 & 7386 & 8222 & 47 \\
\hline $\mathrm{H}(17 \mathrm{~A})$ & 3113 & 7019 & 8683 & 52 \\
\hline $\mathrm{H}(17 \mathrm{~B})$ & 3185 & 6027 & 8550 & 52 \\
\hline
\end{tabular}




\begin{tabular}{|c|c|c|c|c|}
\hline $\mathrm{H}(18 \mathrm{~A})$ & 1991 & 6593 & 8032 & 53 \\
\hline $\mathrm{H}(18 \mathrm{~B})$ & 2492 & 7341 & 7847 & 53 \\
\hline $\mathrm{H}(19 \mathrm{~A})$ & 2624 & 5530 & 7660 & 48 \\
\hline $\mathrm{H}(19 \mathrm{~B})$ & 2257 & 6232 & 7252 & 48 \\
\hline $\mathrm{H}(20 \mathrm{~A})$ & 3532 & 6842 & 7323 & 39 \\
\hline $\mathrm{H}(20 \mathrm{~B})$ & 3572 & 5835 & 7208 & 39 \\
\hline $\mathrm{H}(23)$ & 4098 & 6846 & 5071 & 29 \\
\hline $\mathrm{H}(25)$ & 6432 & 6271 & 5392 & 30 \\
\hline $\mathrm{H}(28 \mathrm{~A})$ & 4074 & 6178 & 4328 & 61 \\
\hline $\mathrm{H}(28 \mathrm{~B})$ & 4460 & 6618 & 3923 & 61 \\
\hline $\mathrm{H}(28 \mathrm{C})$ & 4216 & 7195 & 4336 & 61 \\
\hline $\mathrm{H}(29 \mathrm{~A})$ & 6142 & 5563 & 4683 & 60 \\
\hline $\mathrm{H}(29 \mathrm{~B})$ & 5615 & 5633 & 4130 & 60 \\
\hline $\mathrm{H}(29 \mathrm{C})$ & 5251 & 5176 & 4539 & 60 \\
\hline $\mathrm{H}(30 \mathrm{~A})$ & 5606 & 7794 & 4557 & 55 \\
\hline $\mathrm{H}(30 \mathrm{~B})$ & 5843 & 7205 & 4146 & 55 \\
\hline $\mathrm{H}(30 \mathrm{C})$ & 6358 & 7157 & 4702 & 55 \\
\hline $\mathrm{H}(32 \mathrm{~A})$ & 6561 & 5113 & 6679 & 70 \\
\hline $\mathrm{H}(32 \mathrm{~B})$ & 7496 & 5155 & 6686 & 70 \\
\hline $\mathrm{H}(32 \mathrm{C})$ & 6853 & 4906 & 6192 & 70 \\
\hline $\mathrm{H}(33 \mathrm{~A})$ & 6899 & 7374 & 6716 & 71 \\
\hline H(33B) & 7646 & 6765 & 6946 & 71 \\
\hline $\mathrm{H}(33 \mathrm{C})$ & 6783 & 6565 & 7044 & 71 \\
\hline $\mathrm{H}(34 \mathrm{~A})$ & 7460 & 6980 & 5930 & 67 \\
\hline $\mathrm{H}(34 \mathrm{~B})$ & 7476 & 5982 & 5796 & 67 \\
\hline $\mathrm{H}(34 \mathrm{C})$ & 8054 & 6339 & 6291 & 67 \\
\hline $\mathrm{H}(36 \mathrm{~A})$ & 3262 & 5838 & 5728 & 76 \\
\hline $\mathrm{H}(36 \mathrm{~B})$ & 2818 & 6419 & 6053 & 76 \\
\hline $\mathrm{H}(36 \mathrm{C})$ & 3659 & 5973 & 6299 & 76 \\
\hline $\mathrm{H}(37 \mathrm{~A})$ & 3049 & 6212 & 5234 & 49 \\
\hline $\mathrm{H}(37 \mathrm{~B})$ & 2559 & 6230 & 5652 & 49 \\
\hline $\mathrm{H}(37 \mathrm{C})$ & 3304 & 5589 & 5699 & 49 \\
\hline $\mathrm{H}(38 \mathrm{~A})$ & 3555 & 7991 & 5387 & 69 \\
\hline $\mathrm{H}(38 \mathrm{~B})$ & 2744 & 7742 & 5540 & 69 \\
\hline $\mathrm{H}(38 \mathrm{C})$ & 3059 & 7157 & 5157 & 69 \\
\hline $\mathrm{H}(39 \mathrm{~A})$ & 3758 & 8207 & 5906 & 63 \\
\hline
\end{tabular}




\begin{tabular}{lllll}
$\mathrm{H}(39 \mathrm{~B})$ & 2873 & 7813 & 5735 & 63 \\
$\mathrm{H}(39 \mathrm{C})$ & 3459 & 7804 & 5369 & 63 \\
$\mathrm{H}(40 \mathrm{~A})$ & 4107 & 7429 & 6654 & 71 \\
$\mathrm{H}(40 \mathrm{~B})$ & 3338 & 7937 & 6346 & 71 \\
$\mathrm{H}(40 \mathrm{C})$ & 4211 & 8146 & 6265 & 71 \\
$\mathrm{H}(41 \mathrm{~A})$ & 3713 & 6147 & 6508 & 57 \\
$\mathrm{H}(41 \mathrm{~B})$ & 3016 & 6856 & 6416 & 57 \\
$\mathrm{H}(41 \mathrm{C})$ & 3923 & 7125 & 6664 & 57 \\
$\mathrm{H}(42 \mathrm{~A})$ & 9807 & 6268 & 9187 & 72 \\
$\mathrm{H}(42 \mathrm{~B})$ & 9195 & 6632 & 9488 & 72 \\
$\mathrm{H}(42 \mathrm{C})$ & 9237 & 5621 & 9390 & 72 \\
& & & & \\
\hline
\end{tabular}




\section{Data for $3 b$}

Table S22. Crystal data and structure refinement for Cain27 (3b).

Identification code

Empirical formula

Formula weight

Temperature

Wavelength

Crystal system

Space group

Unit cell dimensions

Volume

Z

Density (calculated)

Absorption coefficient

$\mathrm{F}(000)$

Crystal size

Theta range for data collection

Index ranges

Reflections collected

Independent reflections

Completeness to theta $=25.000^{\circ}$

Absorption correction

Max. and min. transmission

Refinement method

Data / restraints / parameters

Goodness-of-fit on $\mathrm{F}^{2}$

Final $R$ indices [I $>2$ sigma(I)]

$\mathrm{R}$ indices (all data)

Absolute structure parameter

Extinction coefficient

Largest diff. peak and hole
MC2-45

C32 H43 Cl2 P2 Pt

755.59

$100.0 \mathrm{~K}$

$0.71073 \AA$

Orthorhombic

Pca2 1

$\mathrm{a}=12.2583(18) \AA$

$\alpha=90^{\circ}$.

$\mathrm{b}=14.976(2) \AA$

$\beta=90^{\circ}$.

$\mathrm{c}=17.006(2) \AA$

$3122.0(8) \AA^{3}$

4

$1.608 \mathrm{Mg} / \mathrm{m}^{3}$

$4.788 \mathrm{~mm}^{-1}$

1508

$0.2 \times 0.14 \times 0.04 \mathrm{~mm}^{3}$

1.360 to $25.345^{\circ}$.

$-14<=\mathrm{h}<=14,-18<=\mathrm{k}<=16,-18<=1<=20$

35462

$5523[\mathrm{R}($ int $)=0.0378]$

$100.0 \%$

Semi-empirical from equivalents

0.0916 and 0.0568

Full-matrix least-squares on $\mathrm{F}^{2}$

$5523 / 1 / 343$

1.029

$\mathrm{R} 1=0.0285, \mathrm{wR} 2=0.0677$

$\mathrm{R} 1=0.0368, \mathrm{wR} 2=0.0725$

$-0.027(5)$

$\mathrm{n} / \mathrm{a}$

2.372 and -0.702 e. $\AA^{-3}$ 
Table S23. Atomic coordinates ( x 104) and equivalent isotropic displacement parameters $\left(\AA^{2} \times 10^{3}\right)$ for Cain27. $U(e q)$ is defined as one third of the trace of the orthogonalized $U^{i j}$ tensor.

\begin{tabular}{|c|c|c|c|c|}
\hline & $\mathrm{x}$ & $\mathrm{y}$ & Z & $\mathrm{U}(\mathrm{eq})$ \\
\hline $\operatorname{Pt}(1)$ & 4991(1) & $3834(1)$ & $6954(1)$ & $18(1)$ \\
\hline $\mathrm{Cl}(1)$ & $6276(2)$ & $4957(1)$ & $6769(1)$ & $26(1)$ \\
\hline $\mathrm{Cl}(2)$ & $5532(2)$ & $3126(1)$ & $5781(1)$ & $28(1)$ \\
\hline $\mathrm{P}(1)$ & $4474(2)$ & $4478(1)$ & $8078(1)$ & $20(1)$ \\
\hline$P(2)$ & $3701(2)$ & $2852(1)$ & $7208(1)$ & $22(1)$ \\
\hline $\mathrm{C}(1)$ & $5354(7)$ & $4262(5)$ & $8907(5)$ & $22(2)$ \\
\hline$C(2)$ & $6332(7)$ & $3803(5)$ & $8795(5)$ & $22(2)$ \\
\hline$C(3)$ & $7054(7)$ & $3675(6)$ & $9423(5)$ & $29(2)$ \\
\hline$C(4)$ & $6803(7)$ & $4020(6)$ & $10156(5)$ & $26(2)$ \\
\hline$C(5)$ & $5822(6)$ & $4466(6)$ & $10271(5)$ & $26(2)$ \\
\hline$C(6)$ & $5099(6)$ & $4595(6)$ & $9656(5)$ & $22(2)$ \\
\hline$C(7)$ & $4327(6)$ & $5680(5)$ & $8038(5)$ & $24(2)$ \\
\hline $\mathrm{C}(8)$ & $4871(6)$ & $6282(6)$ & $8531(6)$ & $25(2)$ \\
\hline $\mathrm{C}(9)$ & $4670(8)$ & $7181(6)$ & $8478(6)$ & $37(2)$ \\
\hline$C(10)$ & 3933(8) & $7507(6)$ & $7932(6)$ & $39(2)$ \\
\hline$C(11)$ & $3406(7)$ & $6936(6)$ & $7436(5)$ & $33(2)$ \\
\hline $\mathrm{C}(12)$ & $3602(7)$ & $6026(6)$ & $7481(5)$ & $28(2)$ \\
\hline$C(13)$ & $3114(7)$ & $4034(6)$ & $8340(5)$ & $24(2)$ \\
\hline$C(14)$ & $2928(6)$ & $3139(6)$ & $7963(5)$ & $26(2)$ \\
\hline$C(15)$ & $3383(6)$ & $1806(5)$ & $6715(4)$ & $22(2)$ \\
\hline$C(16)$ & $2613(7)$ & 1802(6) & $6086(5)$ & $24(2)$ \\
\hline$C(17)$ & $2433(7)$ & $984(6)$ & $5714(5)$ & $27(2)$ \\
\hline$C(18)$ & $2910(6)$ & $188(6)$ & $5905(5)$ & $24(2)$ \\
\hline$C(19)$ & $3677(7)$ & $225(6)$ & $6513(5)$ & $27(2)$ \\
\hline$C(20)$ & $3934(6)$ & $1002(5)$ & $6927(6)$ & $24(2)$ \\
\hline $\mathrm{C}(21)$ & $1879(6)$ & $2596(5)$ & $5827(5)$ & $25(2)$ \\
\hline$C(22)$ & $2557(7)$ & $3445(5)$ & $5662(5)$ & $28(2)$ \\
\hline$C(23)$ & $992(7)$ & $2750(6)$ & $6455(5)$ & $31(2)$ \\
\hline$C(24)$ & $1279(8)$ & 2397(6) & $5049(5)$ & $33(2)$ \\
\hline$C(25)$ & $2678(7)$ & $-668(6)$ & $5448(5)$ & $31(2)$ \\
\hline$C(26)$ & $1448(7)$ & $-763(6)$ & $5279(6)$ & $33(2)$ \\
\hline
\end{tabular}




\begin{tabular}{lrrrr}
$\mathrm{C}(27)$ & $3035(9)$ & $-1497(6)$ & $5902(7)$ & $47(3)$ \\
$\mathrm{C}(28)$ & $3287(8)$ & $-622(7)$ & $4671(6)$ & $48(3)$ \\
$\mathrm{C}(29)$ & $4753(8)$ & $892(6)$ & $7621(6)$ & $30(2)$ \\
$\mathrm{C}(30)$ & $4082(8)$ & $856(7)$ & $8376(5)$ & $38(2)$ \\
$\mathrm{C}(31)$ & $5611(8)$ & $1644(6)$ & $7661(5)$ & $34(2)$ \\
$\mathrm{C}(32)$ & $5413(8)$ & $18(6)$ & $7560(5)$ & $32(2)$ \\
\hline
\end{tabular}


Table S24. Bond lengths $[\AA]$ and angles $\left[{ }^{\circ}\right]$ for Cain27.

\begin{tabular}{|c|c|}
\hline $\operatorname{Pt}(1)-\mathrm{Cl}(1)$ & $2.3257(19)$ \\
\hline $\mathrm{Pt}(1)-\mathrm{Cl}(2)$ & $2.355(2)$ \\
\hline $\mathrm{Pt}(1)-\mathrm{P}(1)$ & $2.233(2)$ \\
\hline $\mathrm{Pt}(1)-\mathrm{P}(2)$ & $2.201(2)$ \\
\hline $\mathrm{P}(1)-\mathrm{C}(1)$ & $1.804(9)$ \\
\hline $\mathrm{P}(1)-\mathrm{C}(7)$ & $1.810(8)$ \\
\hline$P(1)-C(13)$ & $1.850(9)$ \\
\hline $\mathrm{P}(2)-\mathrm{C}(14)$ & $1.653(9)$ \\
\hline $\mathrm{P}(2)-\mathrm{C}(15)$ & $1.820(8)$ \\
\hline $\mathrm{C}(1)-\mathrm{C}(2)$ & $1.395(12)$ \\
\hline$C(1)-C(6)$ & $1.403(12)$ \\
\hline $\mathrm{C}(2)-\mathrm{H}(2)$ & 0.9500 \\
\hline $\mathrm{C}(2)-\mathrm{C}(3)$ & $1.401(12)$ \\
\hline $\mathrm{C}(3)-\mathrm{H}(3)$ & 0.9500 \\
\hline $\mathrm{C}(3)-\mathrm{C}(4)$ & $1.383(12)$ \\
\hline $\mathrm{C}(4)-\mathrm{H}(4)$ & 0.9500 \\
\hline$C(4)-C(5)$ & $1.390(12)$ \\
\hline $\mathrm{C}(5)-\mathrm{H}(5)$ & 0.9500 \\
\hline $\mathrm{C}(5)-\mathrm{C}(6)$ & $1.385(12)$ \\
\hline $\mathrm{C}(6)-\mathrm{H}(6)$ & 0.9500 \\
\hline$C(7)-C(8)$ & $1.399(12)$ \\
\hline$C(7)-C(12)$ & $1.400(12)$ \\
\hline $\mathrm{C}(8)-\mathrm{H}(8)$ & 0.9500 \\
\hline $\mathrm{C}(8)-\mathrm{C}(9)$ & $1.373(13)$ \\
\hline $\mathrm{C}(9)-\mathrm{H}(9)$ & 0.9500 \\
\hline $\mathrm{C}(9)-\mathrm{C}(10)$ & $1.385(14)$ \\
\hline $\mathrm{C}(10)-\mathrm{H}(10)$ & 0.9500 \\
\hline $\mathrm{C}(10)-\mathrm{C}(11)$ & $1.364(13)$ \\
\hline $\mathrm{C}(11)-\mathrm{H}(11)$ & 0.9500 \\
\hline $\mathrm{C}(11)-\mathrm{C}(12)$ & $1.385(12)$ \\
\hline $\mathrm{C}(12)-\mathrm{H}(12)$ & 0.9500 \\
\hline $\mathrm{C}(13)-\mathrm{H}(13 \mathrm{~A})$ & 0.9900 \\
\hline $\mathrm{C}(13)-\mathrm{H}(13 \mathrm{~B})$ & 0.9900 \\
\hline$C(13)-C(14)$ & $1.503(12)$ \\
\hline
\end{tabular}




\begin{tabular}{|c|c|}
\hline $\mathrm{C}(14)-\mathrm{H}(14)$ & 0.9500 \\
\hline$C(15)-C(16)$ & $1.427(11)$ \\
\hline$C(15)-C(20)$ & $1.428(10)$ \\
\hline$C(16)-C(17)$ & $1.395(12)$ \\
\hline$C(16)-C(21)$ & $1.556(11)$ \\
\hline $\mathrm{C}(17)-\mathrm{H}(17)$ & 0.9500 \\
\hline $\mathrm{C}(17)-\mathrm{C}(18)$ & $1.368(12)$ \\
\hline$C(18)-C(19)$ & $1.398(11)$ \\
\hline$C(18)-C(25)$ & $1.525(12)$ \\
\hline $\mathrm{C}(19)-\mathrm{H}(19)$ & 0.9500 \\
\hline$C(19)-C(20)$ & $1.396(12)$ \\
\hline$C(20)-C(29)$ & $1.558(13)$ \\
\hline$C(21)-C(22)$ & $1.545(11)$ \\
\hline$C(21)-C(23)$ & $1.541(11)$ \\
\hline$C(21)-C(24)$ & $1.543(11)$ \\
\hline $\mathrm{C}(22)-\mathrm{H}(22 \mathrm{~A})$ & 0.9800 \\
\hline $\mathrm{C}(22)-\mathrm{H}(22 \mathrm{~B})$ & 0.9800 \\
\hline $\mathrm{C}(22)-\mathrm{H}(22 \mathrm{C})$ & 0.9800 \\
\hline $\mathrm{C}(23)-\mathrm{H}(23 \mathrm{~A})$ & 0.9800 \\
\hline $\mathrm{C}(23)-\mathrm{H}(23 \mathrm{~B})$ & 0.9800 \\
\hline $\mathrm{C}(23)-\mathrm{H}(23 \mathrm{C})$ & 0.9800 \\
\hline $\mathrm{C}(24)-\mathrm{H}(24 \mathrm{~A})$ & 0.9800 \\
\hline $\mathrm{C}(24)-\mathrm{H}(24 \mathrm{~B})$ & 0.9800 \\
\hline $\mathrm{C}(24)-\mathrm{H}(24 \mathrm{C})$ & 0.9800 \\
\hline$C(25)-C(26)$ & $1.542(12)$ \\
\hline$C(25)-C(27)$ & $1.525(14)$ \\
\hline$C(25)-C(28)$ & $1.520(12)$ \\
\hline $\mathrm{C}(26)-\mathrm{H}(26 \mathrm{~A})$ & 0.9800 \\
\hline $\mathrm{C}(26)-\mathrm{H}(26 \mathrm{~B})$ & 0.9800 \\
\hline $\mathrm{C}(26)-\mathrm{H}(26 \mathrm{C})$ & 0.9800 \\
\hline $\mathrm{C}(27)-\mathrm{H}(27 \mathrm{~A})$ & 0.9800 \\
\hline $\mathrm{C}(27)-\mathrm{H}(27 \mathrm{~B})$ & 0.9800 \\
\hline $\mathrm{C}(27)-\mathrm{H}(27 \mathrm{C})$ & 0.9800 \\
\hline $\mathrm{C}(28)-\mathrm{H}(28 \mathrm{~A})$ & 0.9800 \\
\hline $\mathrm{C}(28)-\mathrm{H}(28 \mathrm{~B})$ & 0.9800 \\
\hline $\mathrm{C}(28)-\mathrm{H}(28 \mathrm{C})$ & 0.9800 \\
\hline
\end{tabular}




\begin{tabular}{|c|c|}
\hline$C(29)-C(30)$ & $1.526(13)$ \\
\hline$C(29)-C(31)$ & $1.543(13)$ \\
\hline$C(29)-C(32)$ & $1.543(13)$ \\
\hline $\mathrm{C}(30)-\mathrm{H}(30 \mathrm{~A})$ & 0.9800 \\
\hline $\mathrm{C}(30)-\mathrm{H}(30 \mathrm{~B})$ & 0.9800 \\
\hline $\mathrm{C}(30)-\mathrm{H}(30 \mathrm{C})$ & 0.9800 \\
\hline $\mathrm{C}(31)-\mathrm{H}(31 \mathrm{~A})$ & 0.9800 \\
\hline $\mathrm{C}(31)-\mathrm{H}(31 \mathrm{~B})$ & 0.9800 \\
\hline $\mathrm{C}(31)-\mathrm{H}(31 \mathrm{C})$ & 0.9800 \\
\hline $\mathrm{C}(32)-\mathrm{H}(32 \mathrm{~A})$ & 0.9800 \\
\hline $\mathrm{C}(32)-\mathrm{H}(32 \mathrm{~B})$ & 0.9800 \\
\hline $\mathrm{C}(32)-\mathrm{H}(32 \mathrm{C})$ & 0.9800 \\
\hline $\mathrm{Cl}(1)-\mathrm{Pt}(1)-\mathrm{Cl}(2)$ & $91.15(7)$ \\
\hline $\mathrm{P}(1)-\mathrm{Pt}(1)-\mathrm{Cl}(1)$ & $89.73(7)$ \\
\hline $\mathrm{P}(1)-\mathrm{Pt}(1)-\mathrm{Cl}(2)$ & $178.89(8)$ \\
\hline $\mathrm{P}(2)-\mathrm{Pt}(1)-\mathrm{Cl}(1)$ & $174.72(8)$ \\
\hline $\mathrm{P}(2)-\mathrm{Pt}(1)-\mathrm{Cl}(2)$ & $93.91(8)$ \\
\hline $\mathrm{P}(2)-\mathrm{Pt}(1)-\mathrm{P}(1)$ & $85.24(8)$ \\
\hline $\mathrm{C}(1)-\mathrm{P}(1)-\mathrm{Pt}(1)$ & 114.9(3) \\
\hline$C(1)-P(1)-C(7)$ & $105.5(4)$ \\
\hline $\mathrm{C}(1)-\mathrm{P}(1)-\mathrm{C}(13)$ & $106.6(4)$ \\
\hline $\mathrm{C}(7)-\mathrm{P}(1)-\mathrm{Pt}(1)$ & $115.2(3)$ \\
\hline $\mathrm{C}(7)-\mathrm{P}(1)-\mathrm{C}(13)$ & $106.1(4)$ \\
\hline $\mathrm{C}(13)-\mathrm{P}(1)-\mathrm{Pt}(1)$ & $107.8(3)$ \\
\hline $\mathrm{C}(14)-\mathrm{P}(2)-\mathrm{Pt}(1)$ & $113.0(3)$ \\
\hline $\mathrm{C}(14)-\mathrm{P}(2)-\mathrm{C}(15)$ & $117.3(4)$ \\
\hline $\mathrm{C}(15)-\mathrm{P}(2)-\mathrm{Pt}(1)$ & 129.7(3) \\
\hline $\mathrm{C}(2)-\mathrm{C}(1)-\mathrm{P}(1)$ & $119.7(6)$ \\
\hline$C(2)-C(1)-C(6)$ & 119.3(8) \\
\hline $\mathrm{C}(6)-\mathrm{C}(1)-\mathrm{P}(1)$ & $120.8(6)$ \\
\hline $\mathrm{C}(1)-\mathrm{C}(2)-\mathrm{H}(2)$ & 119.8 \\
\hline $\mathrm{C}(1)-\mathrm{C}(2)-\mathrm{C}(3)$ & $120.4(8)$ \\
\hline $\mathrm{C}(3)-\mathrm{C}(2)-\mathrm{H}(2)$ & 119.8 \\
\hline $\mathrm{C}(2)-\mathrm{C}(3)-\mathrm{H}(3)$ & 120.1 \\
\hline$C(4)-C(3)-C(2)$ & $119.7(8)$ \\
\hline
\end{tabular}




\begin{tabular}{|c|c|}
\hline $\mathrm{C}(4)-\mathrm{C}(3)-\mathrm{H}(3)$ & 120.1 \\
\hline $\mathrm{C}(3)-\mathrm{C}(4)-\mathrm{H}(4)$ & 120.0 \\
\hline$C(3)-C(4)-C(5)$ & $119.9(8)$ \\
\hline $\mathrm{C}(5)-\mathrm{C}(4)-\mathrm{H}(4)$ & 120.0 \\
\hline $\mathrm{C}(4)-\mathrm{C}(5)-\mathrm{H}(5)$ & 119.5 \\
\hline$C(6)-C(5)-C(4)$ & $120.9(8)$ \\
\hline $\mathrm{C}(6)-\mathrm{C}(5)-\mathrm{H}(5)$ & 119.5 \\
\hline $\mathrm{C}(1)-\mathrm{C}(6)-\mathrm{H}(6)$ & 120.2 \\
\hline$C(5)-C(6)-C(1)$ & $119.7(8)$ \\
\hline $\mathrm{C}(5)-\mathrm{C}(6)-\mathrm{H}(6)$ & 120.2 \\
\hline $\mathrm{C}(8)-\mathrm{C}(7)-\mathrm{P}(1)$ & $124.8(7)$ \\
\hline $\mathrm{C}(8)-\mathrm{C}(7)-\mathrm{C}(12)$ & $118.0(8)$ \\
\hline $\mathrm{C}(12)-\mathrm{C}(7)-\mathrm{P}(1)$ & $117.2(6)$ \\
\hline $\mathrm{C}(7)-\mathrm{C}(8)-\mathrm{H}(8)$ & 119.8 \\
\hline$C(9)-C(8)-C(7)$ & $120.5(9)$ \\
\hline $\mathrm{C}(9)-\mathrm{C}(8)-\mathrm{H}(8)$ & 119.8 \\
\hline $\mathrm{C}(8)-\mathrm{C}(9)-\mathrm{H}(9)$ & 119.8 \\
\hline $\mathrm{C}(8)-\mathrm{C}(9)-\mathrm{C}(10)$ & $120.5(10)$ \\
\hline $\mathrm{C}(10)-\mathrm{C}(9)-\mathrm{H}(9)$ & 119.8 \\
\hline $\mathrm{C}(9)-\mathrm{C}(10)-\mathrm{H}(10)$ & 119.9 \\
\hline $\mathrm{C}(11)-\mathrm{C}(10)-\mathrm{C}(9)$ & $120.1(9)$ \\
\hline $\mathrm{C}(11)-\mathrm{C}(10)-\mathrm{H}(10)$ & 119.9 \\
\hline $\mathrm{C}(10)-\mathrm{C}(11)-\mathrm{H}(11)$ & 120.0 \\
\hline$C(10)-C(11)-C(12)$ & $120.1(9)$ \\
\hline $\mathrm{C}(12)-\mathrm{C}(11)-\mathrm{H}(11)$ & 120.0 \\
\hline $\mathrm{C}(7)-\mathrm{C}(12)-\mathrm{H}(12)$ & 119.6 \\
\hline $\mathrm{C}(11)-\mathrm{C}(12)-\mathrm{C}(7)$ & $120.7(8)$ \\
\hline $\mathrm{C}(11)-\mathrm{C}(12)-\mathrm{H}(12)$ & 119.6 \\
\hline $\mathrm{P}(1)-\mathrm{C}(13)-\mathrm{H}(13 \mathrm{~A})$ & 109.5 \\
\hline $\mathrm{P}(1)-\mathrm{C}(13)-\mathrm{H}(13 \mathrm{~B})$ & 109.5 \\
\hline $\mathrm{H}(13 \mathrm{~A})-\mathrm{C}(13)-\mathrm{H}(13 \mathrm{~B})$ & 108.1 \\
\hline $\mathrm{C}(14)-\mathrm{C}(13)-\mathrm{P}(1)$ & $110.8(5)$ \\
\hline $\mathrm{C}(14)-\mathrm{C}(13)-\mathrm{H}(13 \mathrm{~A})$ & 109.5 \\
\hline $\mathrm{C}(14)-\mathrm{C}(13)-\mathrm{H}(13 \mathrm{~B})$ & 109.5 \\
\hline $\mathrm{P}(2)-\mathrm{C}(14)-\mathrm{H}(14)$ & 120.8 \\
\hline $\mathrm{C}(13)-\mathrm{C}(14)-\mathrm{P}(2)$ & $118.4(6)$ \\
\hline
\end{tabular}




\begin{tabular}{|c|c|}
\hline $\mathrm{C}(13)-\mathrm{C}(14)-\mathrm{H}(14)$ & 120.8 \\
\hline $\mathrm{C}(16)-\mathrm{C}(15)-\mathrm{P}(2)$ & $119.4(6)$ \\
\hline$C(16)-C(15)-C(20)$ & $119.9(7)$ \\
\hline $\mathrm{C}(20)-\mathrm{C}(15)-\mathrm{P}(2)$ & $120.6(6)$ \\
\hline$C(15)-C(16)-C(21)$ & $126.2(7)$ \\
\hline$C(17)-C(16)-C(15)$ & $116.6(8)$ \\
\hline$C(17)-C(16)-C(21)$ & $116.9(7)$ \\
\hline $\mathrm{C}(16)-\mathrm{C}(17)-\mathrm{H}(17)$ & 116.9 \\
\hline$C(18)-C(17)-C(16)$ & $126.2(9)$ \\
\hline $\mathrm{C}(18)-\mathrm{C}(17)-\mathrm{H}(17)$ & 116.9 \\
\hline$C(17)-C(18)-C(19)$ & $115.3(8)$ \\
\hline$C(17)-C(18)-C(25)$ & $122.1(8)$ \\
\hline$C(19)-C(18)-C(25)$ & $122.4(7)$ \\
\hline $\mathrm{C}(18)-\mathrm{C}(19)-\mathrm{H}(19)$ & 118.0 \\
\hline$C(20)-C(19)-C(18)$ & $123.9(8)$ \\
\hline $\mathrm{C}(20)-\mathrm{C}(19)-\mathrm{H}(19)$ & 118.0 \\
\hline$C(15)-C(20)-C(29)$ & $125.8(7)$ \\
\hline$C(19)-C(20)-C(15)$ & $118.0(8)$ \\
\hline$C(19)-C(20)-C(29)$ & $116.1(7)$ \\
\hline$C(22)-C(21)-C(16)$ & $111.7(7)$ \\
\hline$C(22)-C(21)-C(23)$ & $112.6(7)$ \\
\hline$C(23)-C(21)-C(16)$ & $109.0(7)$ \\
\hline$C(24)-C(21)-C(16)$ & $111.7(7)$ \\
\hline$C(24)-C(21)-C(22)$ & $105.1(7)$ \\
\hline$C(24)-C(21)-C(23)$ & $106.7(7)$ \\
\hline $\mathrm{C}(21)-\mathrm{C}(22)-\mathrm{H}(22 \mathrm{~A})$ & 109.5 \\
\hline $\mathrm{C}(21)-\mathrm{C}(22)-\mathrm{H}(22 \mathrm{~B})$ & 109.5 \\
\hline $\mathrm{C}(21)-\mathrm{C}(22)-\mathrm{H}(22 \mathrm{C})$ & 109.5 \\
\hline $\mathrm{H}(22 \mathrm{~A})-\mathrm{C}(22)-\mathrm{H}(22 \mathrm{~B})$ & 109.5 \\
\hline $\mathrm{H}(22 \mathrm{~A})-\mathrm{C}(22)-\mathrm{H}(22 \mathrm{C})$ & 109.5 \\
\hline $\mathrm{H}(22 \mathrm{~B})-\mathrm{C}(22)-\mathrm{H}(22 \mathrm{C})$ & 109.5 \\
\hline $\mathrm{C}(21)-\mathrm{C}(23)-\mathrm{H}(23 \mathrm{~A})$ & 109.5 \\
\hline $\mathrm{C}(21)-\mathrm{C}(23)-\mathrm{H}(23 \mathrm{~B})$ & 109.5 \\
\hline $\mathrm{C}(21)-\mathrm{C}(23)-\mathrm{H}(23 \mathrm{C})$ & 109.5 \\
\hline $\mathrm{H}(23 \mathrm{~A})-\mathrm{C}(23)-\mathrm{H}(23 \mathrm{~B})$ & 109.5 \\
\hline $\mathrm{H}(23 \mathrm{~A})-\mathrm{C}(23)-\mathrm{H}(23 \mathrm{C})$ & 109.5 \\
\hline
\end{tabular}




\begin{tabular}{|c|c|}
\hline $\mathrm{H}(23 \mathrm{~B})-\mathrm{C}(23)-\mathrm{H}(23 \mathrm{C})$ & 109.5 \\
\hline $\mathrm{C}(21)-\mathrm{C}(24)-\mathrm{H}(24 \mathrm{~A})$ & 109.5 \\
\hline $\mathrm{C}(21)-\mathrm{C}(24)-\mathrm{H}(24 \mathrm{~B})$ & 109.5 \\
\hline $\mathrm{C}(21)-\mathrm{C}(24)-\mathrm{H}(24 \mathrm{C})$ & 109.5 \\
\hline $\mathrm{H}(24 \mathrm{~A})-\mathrm{C}(24)-\mathrm{H}(24 \mathrm{~B})$ & 109.5 \\
\hline $\mathrm{H}(24 \mathrm{~A})-\mathrm{C}(24)-\mathrm{H}(24 \mathrm{C})$ & 109.5 \\
\hline $\mathrm{H}(24 \mathrm{~B})-\mathrm{C}(24)-\mathrm{H}(24 \mathrm{C})$ & 109.5 \\
\hline $\mathrm{C}(18)-\mathrm{C}(25)-\mathrm{C}(26)$ & $110.8(7)$ \\
\hline $\mathrm{C}(27)-\mathrm{C}(25)-\mathrm{C}(18)$ & $111.9(7)$ \\
\hline $\mathrm{C}(27)-\mathrm{C}(25)-\mathrm{C}(26)$ & $107.5(8)$ \\
\hline $\mathrm{C}(27)-\mathrm{C}(25)-\mathrm{C}(28)$ & $109.7(8)$ \\
\hline $\mathrm{C}(28)-\mathrm{C}(25)-\mathrm{C}(18)$ & $108.2(7)$ \\
\hline $\mathrm{C}(28)-\mathrm{C}(25)-\mathrm{C}(26)$ & $108.8(8)$ \\
\hline $\mathrm{C}(25)-\mathrm{C}(26)-\mathrm{H}(26 \mathrm{~A})$ & 109.5 \\
\hline $\mathrm{C}(25)-\mathrm{C}(26)-\mathrm{H}(26 \mathrm{~B})$ & 109.5 \\
\hline $\mathrm{C}(25)-\mathrm{C}(26)-\mathrm{H}(26 \mathrm{C})$ & 109.5 \\
\hline $\mathrm{H}(26 \mathrm{~A})-\mathrm{C}(26)-\mathrm{H}(26 \mathrm{~B})$ & 109.5 \\
\hline$H(26 A)-C(26)-H(26 C)$ & 109.5 \\
\hline $\mathrm{H}(26 \mathrm{~B})-\mathrm{C}(26)-\mathrm{H}(26 \mathrm{C})$ & 109.5 \\
\hline $\mathrm{C}(25)-\mathrm{C}(27)-\mathrm{H}(27 \mathrm{~A})$ & 109.5 \\
\hline $\mathrm{C}(25)-\mathrm{C}(27)-\mathrm{H}(27 \mathrm{~B})$ & 109.5 \\
\hline $\mathrm{C}(25)-\mathrm{C}(27)-\mathrm{H}(27 \mathrm{C})$ & 109.5 \\
\hline $\mathrm{H}(27 \mathrm{~A})-\mathrm{C}(27)-\mathrm{H}(27 \mathrm{~B})$ & 109.5 \\
\hline $\mathrm{H}(27 \mathrm{~A})-\mathrm{C}(27)-\mathrm{H}(27 \mathrm{C})$ & 109.5 \\
\hline $\mathrm{H}(27 \mathrm{~B})-\mathrm{C}(27)-\mathrm{H}(27 \mathrm{C})$ & 109.5 \\
\hline $\mathrm{C}(25)-\mathrm{C}(28)-\mathrm{H}(28 \mathrm{~A})$ & 109.5 \\
\hline $\mathrm{C}(25)-\mathrm{C}(28)-\mathrm{H}(28 \mathrm{~B})$ & 109.5 \\
\hline $\mathrm{C}(25)-\mathrm{C}(28)-\mathrm{H}(28 \mathrm{C})$ & 109.5 \\
\hline $\mathrm{H}(28 \mathrm{~A})-\mathrm{C}(28)-\mathrm{H}(28 \mathrm{~B})$ & 109.5 \\
\hline $\mathrm{H}(28 \mathrm{~A})-\mathrm{C}(28)-\mathrm{H}(28 \mathrm{C})$ & 109.5 \\
\hline $\mathrm{H}(28 \mathrm{~B})-\mathrm{C}(28)-\mathrm{H}(28 \mathrm{C})$ & 109.5 \\
\hline $\mathrm{C}(30)-\mathrm{C}(29)-\mathrm{C}(20)$ & $107.1(7)$ \\
\hline $\mathrm{C}(30)-\mathrm{C}(29)-\mathrm{C}(31)$ & $110.8(8)$ \\
\hline $\mathrm{C}(30)-\mathrm{C}(29)-\mathrm{C}(32)$ & $108.0(8)$ \\
\hline $\mathrm{C}(31)-\mathrm{C}(29)-\mathrm{C}(20)$ & $113.4(7)$ \\
\hline $\mathrm{C}(31)-\mathrm{C}(29)-\mathrm{C}(32)$ & $105.4(8)$ \\
\hline
\end{tabular}




$\begin{array}{ll}\mathrm{C}(32)-\mathrm{C}(29)-\mathrm{C}(20) & 112.1(8) \\ \mathrm{C}(29)-\mathrm{C}(30)-\mathrm{H}(30 \mathrm{~A}) & 109.5 \\ \mathrm{C}(29)-\mathrm{C}(30)-\mathrm{H}(30 \mathrm{~B}) & 109.5 \\ \mathrm{C}(29)-\mathrm{C}(30)-\mathrm{H}(30 \mathrm{C}) & 109.5 \\ \mathrm{H}(30 \mathrm{~A})-\mathrm{C}(30)-\mathrm{H}(30 \mathrm{~B}) & 109.5 \\ \mathrm{H}(30 \mathrm{~A})-\mathrm{C}(30)-\mathrm{H}(30 \mathrm{C}) & 109.5 \\ \mathrm{H}(30 \mathrm{~B})-\mathrm{C}(30)-\mathrm{H}(30 \mathrm{C}) & 109.5 \\ \mathrm{C}(29)-\mathrm{C}(31)-\mathrm{H}(31 \mathrm{~A}) & 109.5 \\ \mathrm{C}(29)-\mathrm{C}(31)-\mathrm{H}(31 \mathrm{~B}) & 109.5 \\ \mathrm{C}(29)-\mathrm{C}(31)-\mathrm{H}(31 \mathrm{C}) & 109.5 \\ \mathrm{H}(31 \mathrm{~A})-\mathrm{C}(31)-\mathrm{H}(31 \mathrm{~B}) & 109.5 \\ \mathrm{H}(31 \mathrm{~A})-\mathrm{C}(31)-\mathrm{H}(31 \mathrm{C}) & 109.5 \\ \mathrm{H}(31 \mathrm{~B})-\mathrm{C}(31)-\mathrm{H}(31 \mathrm{C}) & 109.5 \\ \mathrm{C}(29)-\mathrm{C}(32)-\mathrm{H}(32 \mathrm{~A}) & 109.5 \\ \mathrm{C}(29)-\mathrm{C}(32)-\mathrm{H}(32 \mathrm{~B}) & 109.5 \\ \mathrm{C}(29)-\mathrm{C}(32)-\mathrm{H}(32 \mathrm{C}) & 109.5 \\ \mathrm{H}(32 \mathrm{~A})-\mathrm{C}(32)-\mathrm{H}(32 \mathrm{~B}) & 109.5 \\ \mathrm{H}(32 \mathrm{~A})-\mathrm{C}(32)-\mathrm{H}(32 \mathrm{C}) & 109.5 \\ \mathrm{H}(32 \mathrm{~B})-\mathrm{C}(32)-\mathrm{H}(32 \mathrm{C}) & 109.5\end{array}$

Symmetry transformations used to generate equivalent atoms: 
Table S25. Anisotropic displacement parameters $\left(\AA^{2} \times 10^{3}\right)$ for Cain27. The anisotropic displacement factor exponent takes the form: $-2 \pi^{2}\left[h^{2} a^{* 2} U^{11}+\ldots+2 h k a^{*} b^{*} U^{12}\right]$

\begin{tabular}{|c|c|c|c|c|c|c|}
\hline & $\mathrm{U}^{11}$ & $\mathrm{U}^{22}$ & $\mathrm{U}^{33}$ & $\mathrm{U}^{23}$ & $\mathrm{U}^{13}$ & $\mathrm{U}^{12}$ \\
\hline $\operatorname{Pt}(1)$ & $17(1)$ & $20(1)$ & $16(1)$ & $0(1)$ & $1(1)$ & $-1(1)$ \\
\hline $\mathrm{Cl}(1)$ & 21(1) & $28(1)$ & $29(1)$ & $3(1)$ & $1(1)$ & $-4(1)$ \\
\hline $\mathrm{Cl}(2)$ & $28(1)$ & $33(1)$ & $23(1)$ & $-4(1)$ & $5(1)$ & $-2(1)$ \\
\hline $\mathrm{P}(1)$ & $18(1)$ & $23(1)$ & $20(1)$ & $0(1)$ & $1(1)$ & $-3(1)$ \\
\hline $\mathrm{P}(2)$ & $23(1)$ & $23(1)$ & $21(1)$ & $-1(1)$ & $1(1)$ & $-3(1)$ \\
\hline$C(1)$ & $25(4)$ & $17(4)$ & $24(5)$ & $2(3)$ & $0(3)$ & $-3(3)$ \\
\hline$C(2)$ & $17(4)$ & $32(5)$ & $15(4)$ & $-1(3)$ & $0(3)$ & $-2(3)$ \\
\hline$C(3)$ & $22(4)$ & $38(5)$ & $27(5)$ & $2(4)$ & $7(4)$ & $4(4)$ \\
\hline$C(4)$ & $26(5)$ & $34(5)$ & $19(4)$ & $0(4)$ & $-6(4)$ & $-3(4)$ \\
\hline$C(5)$ & 21(4) & $32(5)$ & $25(5)$ & $-5(4)$ & $1(3)$ & $-5(4)$ \\
\hline$C(6)$ & $24(4)$ & $19(4)$ & $21(4)$ & $1(3)$ & $2(3)$ & $0(3)$ \\
\hline$C(7)$ & 21(4) & $28(5)$ & $23(4)$ & $-4(4)$ & $8(4)$ & $-2(3)$ \\
\hline$C(8)$ & $23(5)$ & $27(5)$ & $26(5)$ & $-3(4)$ & $1(3)$ & $-1(3)$ \\
\hline $\mathrm{C}(9)$ & $43(5)$ & $27(5)$ & $40(6)$ & $-1(4)$ & $13(5)$ & $-6(4)$ \\
\hline$C(10)$ & $45(6)$ & $25(5)$ & $47(6)$ & $6(5)$ & $13(5)$ & $3(4)$ \\
\hline $\mathrm{C}(11)$ & $30(5)$ & $30(5)$ & $38(6)$ & $11(4)$ & $6(4)$ & $10(4)$ \\
\hline$C(12)$ & $23(5)$ & $35(5)$ & $25(5)$ & $2(4)$ & $5(4)$ & $-2(4)$ \\
\hline $\mathrm{C}(13)$ & 21(4) & $29(5)$ & $22(4)$ & $-2(4)$ & $-1(4)$ & $6(4)$ \\
\hline$C(14)$ & $22(5)$ & $34(5)$ & $23(5)$ & $5(4)$ & $-2(3)$ & $-8(4)$ \\
\hline$C(15)$ & $20(4)$ & $26(4)$ & $20(4)$ & $-2(3)$ & $3(3)$ & $-1(3)$ \\
\hline$C(16)$ & $25(4)$ & $26(5)$ & $22(4)$ & $6(4)$ & $0(3)$ & $0(3)$ \\
\hline $\mathrm{C}(17)$ & $23(5)$ & $34(5)$ & $23(5)$ & $5(4)$ & $-1(4)$ & $0(4)$ \\
\hline$C(18)$ & $19(4)$ & $31(5)$ & $23(5)$ & $0(4)$ & $5(4)$ & $-4(3)$ \\
\hline $\mathrm{C}(19)$ & $28(5)$ & $26(5)$ & $26(5)$ & $1(3)$ & $5(4)$ & $5(4)$ \\
\hline$C(20)$ & $25(4)$ & $25(4)$ & $22(4)$ & $4(4)$ & $3(4)$ & $-3(3)$ \\
\hline $\mathrm{C}(21)$ & $24(4)$ & $25(5)$ & $26(4)$ & $-2(4)$ & $-4(4)$ & 1(3) \\
\hline$C(22)$ & $28(5)$ & $28(5)$ & $27(5)$ & $2(4)$ & $-1(4)$ & 1(4) \\
\hline$C(23)$ & $27(5)$ & $31(5)$ & $33(5)$ & $3(4)$ & $3(4)$ & $-1(4)$ \\
\hline$C(24)$ & $37(5)$ & $38(5)$ & $23(5)$ & $-2(4)$ & $-5(4)$ & $7(4)$ \\
\hline$C(25)$ & $32(5)$ & $31(5)$ & $29(5)$ & $-7(4)$ & $0(4)$ & $-4(4)$ \\
\hline$C(26)$ & $36(5)$ & $28(5)$ & $36(5)$ & $-3(4)$ & $-8(4)$ & $-3(4)$ \\
\hline
\end{tabular}




\begin{tabular}{lllllll}
$\mathrm{C}(27)$ & $52(7)$ & $28(5)$ & $60(7)$ & $-18(5)$ & $-13(6)$ & $7(5)$ \\
$\mathrm{C}(28)$ & $48(6)$ & $56(7)$ & $41(6)$ & $-25(6)$ & $16(5)$ & $-13(5)$ \\
$\mathrm{C}(29)$ & $43(6)$ & $20(4)$ & $27(5)$ & $5(4)$ & $-4(4)$ & $-2(4)$ \\
$\mathrm{C}(30)$ & $31(5)$ & $55(6)$ & $26(5)$ & $5(5)$ & $-4(4)$ & $3(5)$ \\
$\mathrm{C}(31)$ & $36(6)$ & $33(5)$ & $34(5)$ & $0(4)$ & $-10(4)$ & $-13(4)$ \\
$\mathrm{C}(32)$ & $27(5)$ & $37(5)$ & $33(5)$ & $-1(4)$ & $-10(4)$ & $0(4)$ \\
\hline
\end{tabular}


Table S26. Hydrogen coordinates ( x 10 $)$ and isotropic displacement parameters $\left(\AA^{2} \times 10^{3}\right)$ for Cain27.

\begin{tabular}{|c|c|c|c|c|}
\hline & $\mathrm{x}$ & $\mathrm{y}$ & $\mathrm{z}$ & $\mathrm{U}(\mathrm{eq})$ \\
\hline $\mathrm{H}(2)$ & 6510 & 3577 & 8289 & 26 \\
\hline $\mathrm{H}(3)$ & 7713 & 3352 & 9347 & 34 \\
\hline $\mathrm{H}(4)$ & 7302 & 3952 & 10579 & 32 \\
\hline $\mathrm{H}(5)$ & 5644 & 4686 & 10779 & 31 \\
\hline $\mathrm{H}(6)$ & 4435 & 4906 & 9740 & 26 \\
\hline $\mathrm{H}(8)$ & 5383 & 6066 & 8905 & 30 \\
\hline $\mathrm{H}(9)$ & 5039 & 7583 & 8819 & 44 \\
\hline $\mathrm{H}(10)$ & 3794 & 8131 & 7903 & 47 \\
\hline $\mathrm{H}(11)$ & 2903 & 7162 & 7060 & 39 \\
\hline $\mathrm{H}(12)$ & 3240 & 5633 & 7128 & 33 \\
\hline $\mathrm{H}(13 \mathrm{~A})$ & 3058 & 3975 & 8919 & 29 \\
\hline $\mathrm{H}(13 \mathrm{~B})$ & 2543 & 4456 & 8163 & 29 \\
\hline $\mathrm{H}(14)$ & 2379 & 2747 & 8155 & 32 \\
\hline $\mathrm{H}(17)$ & 1931 & 981 & 5289 & 32 \\
\hline H(19) & 4045 & -310 & 6652 & 32 \\
\hline $\mathrm{H}(22 \mathrm{~A})$ & 3138 & 3305 & 5285 & 42 \\
\hline $\mathrm{H}(22 \mathrm{~B})$ & 2882 & 3660 & 6153 & 42 \\
\hline $\mathrm{H}(22 \mathrm{C})$ & 2083 & 3909 & 5442 & 42 \\
\hline$H(23 A)$ & 1333 & 2956 & 6943 & 46 \\
\hline $\mathrm{H}(23 \mathrm{~B})$ & 603 & 2189 & 6553 & 46 \\
\hline $\mathrm{H}(23 \mathrm{C})$ & 475 & 3201 & 6267 & 46 \\
\hline $\mathrm{H}(24 \mathrm{~A})$ & 786 & 1889 & 5124 & 49 \\
\hline $\mathrm{H}(24 \mathrm{~B})$ & 1814 & 2252 & 4640 & 49 \\
\hline $\mathrm{H}(24 \mathrm{C})$ & 857 & 2922 & 4890 & 49 \\
\hline$H(26 A)$ & 1200 & -255 & 4964 & 50 \\
\hline $\mathrm{H}(26 \mathrm{~B})$ & 1045 & -777 & 5777 & 50 \\
\hline $\mathrm{H}(26 \mathrm{C})$ & 1316 & -1319 & 4989 & 50 \\
\hline $\mathrm{H}(27 \mathrm{~A})$ & 2713 & -1486 & 6429 & 70 \\
\hline $\mathrm{H}(27 \mathrm{~B})$ & 3832 & -1504 & 5945 & 70 \\
\hline $\mathrm{H}(27 \mathrm{C})$ & 2789 & -2033 & 5623 & 70 \\
\hline
\end{tabular}




\begin{tabular}{lrrrr}
$\mathrm{H}(28 \mathrm{~A})$ & 4074 & -603 & 4771 & 72 \\
$\mathrm{H}(28 \mathrm{~B})$ & 3067 & -82 & 4386 & 72 \\
$\mathrm{H}(28 \mathrm{C})$ & 3112 & -1150 & 4354 & 72 \\
$\mathrm{H}(30 \mathrm{~A})$ & 3704 & 1426 & 8451 & 56 \\
$\mathrm{H}(30 \mathrm{~B})$ & 4567 & 745 & 8824 & 56 \\
$\mathrm{H}(30 \mathrm{C})$ & 3545 & 373 & 8338 & 56 \\
$\mathrm{H}(31 \mathrm{~A})$ & 6231 & 1447 & 7982 & 51 \\
$\mathrm{H}(31 \mathrm{~B})$ & 5284 & 2177 & 7899 & 51 \\
$\mathrm{H}(31 \mathrm{C})$ & 5865 & 1787 & 7129 & 51 \\
$\mathrm{H}(32 \mathrm{~A})$ & 5959 & -1 & 7981 & 48 \\
$\mathrm{H}(32 \mathrm{~B})$ & 5781 & -8 & 7049 & 48 \\
$\mathrm{H}(32 \mathrm{C})$ & 4920 & -494 & 7611 & 48 \\
& & & & \\
\hline
\end{tabular}

